\title{
The impact of age on the elicitation of spreading depolarization and the implication of prostanoids in the associated cerebral blood flow response
}

\author{
PhD Thesis
}

Dr. Hertelendy, Péter 



\section{University of Szeged}

Faculty of Medicine, Faculty of Science and Informatics

Department of Medical Physics and Informatics

The impact of age on the elicitation of spreading depolarization and the implication of prostanoids in the associated cerebral blood

flow response

Dr. Hertelendy, Péter

$\mathrm{PhD}$ Thesis

Supervisor: Dr. Farkas, Eszter, Dr. Bari, Ferenc

Doctoral School of Theoretical Medicine

Szeged, 2019 



\section{Contents}

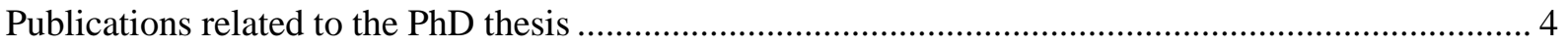

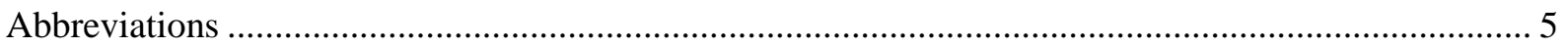

1. Introduction

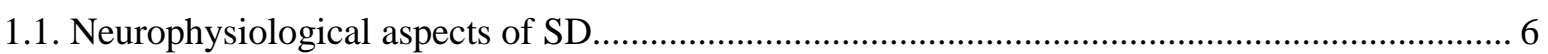

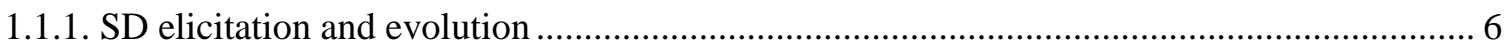

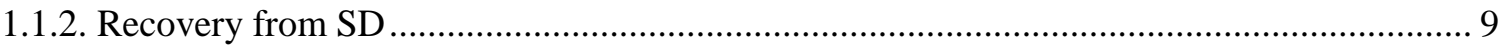

1.1.3. Cerebral blood flow response to spreading depolarization.................................................. 10

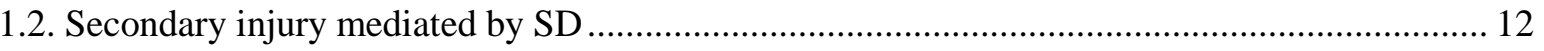

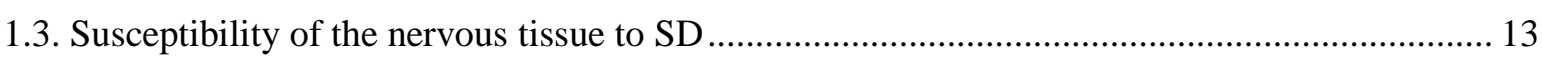

1.4. Age as a risk factor for neurological diseases in which SD is relevant .................................... 14

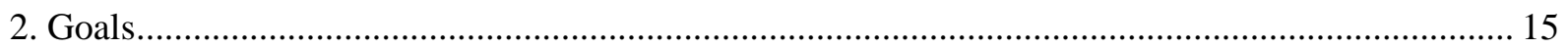

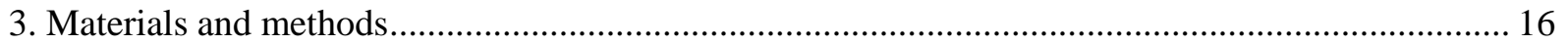

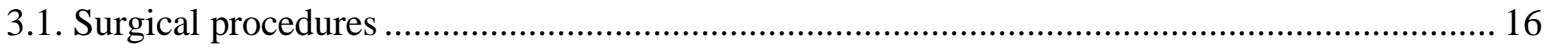

3.2. Recording of electrophysiological parameters and cerebral blood flow ................................... 17

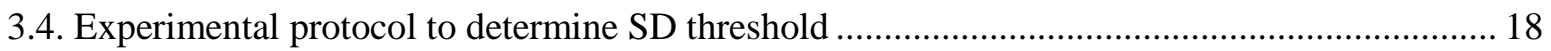

3.5. Experimental protocol for pharmacological investigation ..................................................... 19

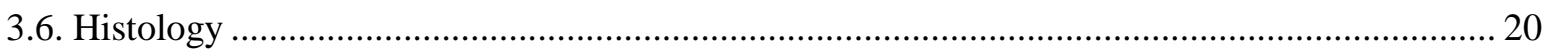

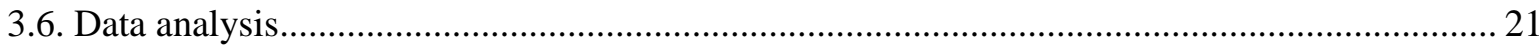

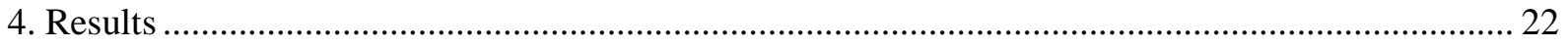

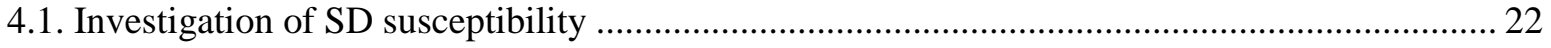

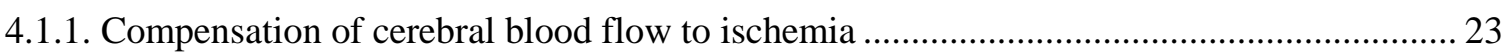

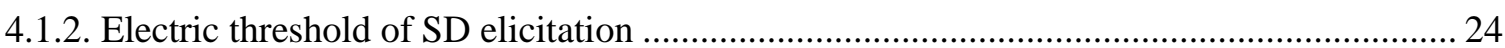

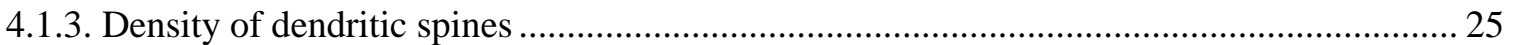

4.1.4. Features of the cerebral blood flow response associated with SD ...................................... 26

4.1.5. Spectral analysis of the distinct ECoG frequency bands ................................................... 28

4.2. Pharmacological manipulations to explore potential mediators of the cerebral blood flow

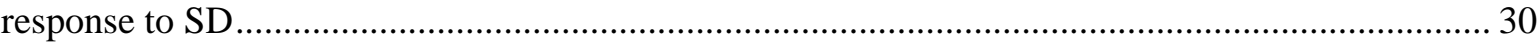

4.2.1. Effect of pharmacological manipulation on systemic physiological variables and baseline

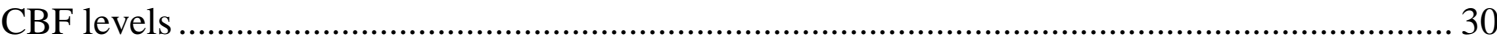

4.2.2. Pharmacological manipulation of SD related cerebral blood flow response ....................... 31

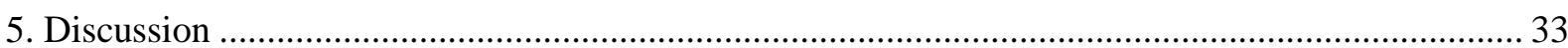

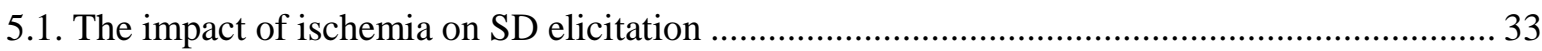

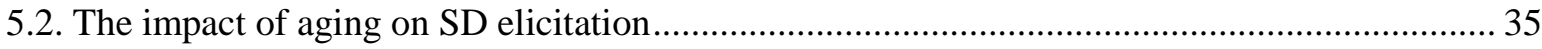

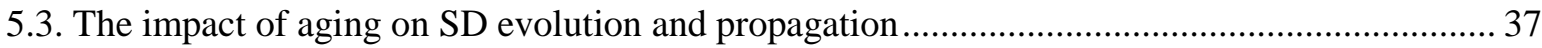

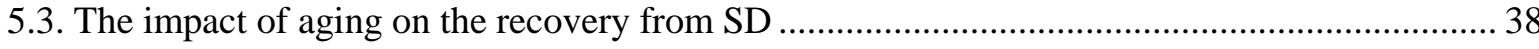

5.4. SDs occurring spontaneously in response to hypoxia/ischemia .................................................. 40 


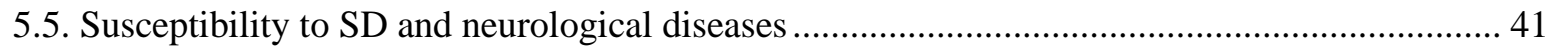

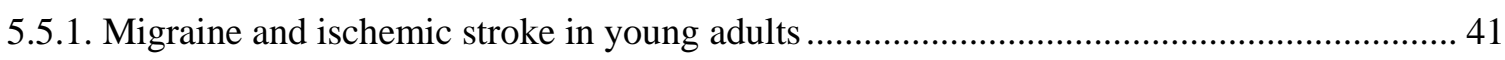

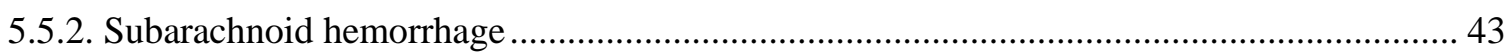

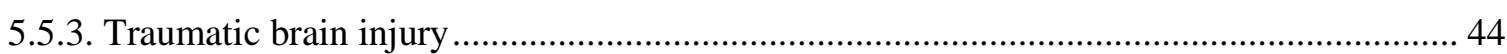

5.6. Role of prostanoids in the cerebral blood flow response to SD ................................................ 46

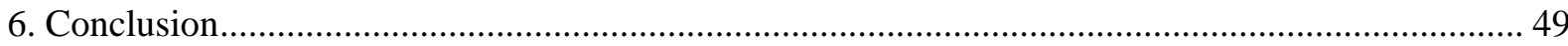




\section{Publications related to the $\mathrm{PhD}$ thesis}

I. Hertelendy, P., Varga, D. P., Menyhárt, Á., Bari, F., \& Farkas, E.

Susceptibility of the cerebral cortex to spreading depolarization in neurological disease states: The impact of aging.

Neurochemistry International. 2018 In press. doi:10.1016/j.neuint.2018.10.010

IF: 3.603

II. Hertelendy, P., Menyhárt, Á., Makra, P., Süle, Z., Kiss, T., Tóth, G., Ivánkovits-Kiss, O, Bari, F, Farkas, E.

Advancing age and ischemia elevate the electric threshold to elicit spreading depolarization in the cerebral cortex of young adult rats.

J Cereb Blood Flow Metab. 2017 May;37(5):1763-1775. doi:

$10.1177 / 0271678 X 16643735$

\section{IF: $\mathbf{5 . 4 0 7}$}

III. Varga, D.P., Puskás, T., Menyhárt, Á., Hertelendy, P., Zölei-Szénási, D., Tóth, R., Ivánkovits-Kiss, O., Bari, F., Farkas, E.

Contribution of prostanoid signaling to the evolution of spreading depolarization and the associated cerebral blood flow response.

Sci Rep. 2016 Aug 10;6:31402. doi: 10.1038/srep31402.

IF: $\mathbf{5 . 5 2 5}$ 


\section{Abbreviations}

20-HETE: 20-Hydroxyeicosatetraenoic acid

2VO: 2-vessel occlusion (transient bilateral common carotid artery occlusion)

aCSF: artificial cerebrospinal fluid

$\mathrm{Ag} / \mathrm{AgCl}$ : silver/silver-chloride

AMPA: $\alpha$-amino-3-hydroxy-5-methyl-4-isoxazole propionate

ANOVA: Analysis of variance

ATP: Adenosine-tryphosphate

BK: large-conductance $\mathrm{Ca}^{2+}$ activated $\mathrm{K}^{+}$channel

$\mathrm{Ca}^{2}$ : calcium ion

Cav: voltage-gated calcium channel

CBF: cerebral blood flow

$\mathrm{Cl}^{-}$: chloride ion

$\mathrm{CO}_{2}$ : carbon-dioxide

COX-1: cyclooxygenase-1

COX-2: cyclooxygenase-2

DC potential: direct current potential

DCI: delayed cerebral ischemia

DMSO: Dimethyl sulfoxide

EAAT: excitatory amino acid transporter

ECoG: electrocorticogram

eNOS: endothelial nitric oxide synthase

FHM: Familial hemiplegic migraine

fMRI-BOLD: functional magnetic resonance imaging using blood oxygen level detection

GFAP: glial fibrillary acidic protein

IL-1 $\beta$ : interleukin-1 $\beta$

$\mathrm{K}^{+}$: potassium ion

$\mathrm{KCl}$ : potassium chloride

Kir: inward rectifier potassium channel

Kv: voltage-gated potassium channel

MABP: mean arterial blood pressure

mRNA: messenger Ribonucleic acid

$\mathrm{N}_{2} \mathrm{O}$ : nitrous oxide

$\mathrm{Na}^{+}$: sodium ion

NADH: Nicotinamide adenine dinucleotide

Nav: voltage-gated sodium channel

NMDA: N-methyl-D-aspartate

nNOS: neuronal nitric oxide synthase

NO: nitric oxide

$\mathrm{O}_{2}$ : molecular oxygen

PET: positron-emission tomography

$\mathrm{PGE}_{2}$ : prostaglandin $\mathrm{E}_{2}$

$\mathrm{PGF}_{2 \alpha}$ : prostaglandin $\mathrm{F}_{2 \alpha}$

SAH: subarachnoid hemorrhage

SD: spreading depolarization

TBI: traumatic brain injury 


\section{Introduction}

Spreading depolarization (SD) is a wave of massive depolarization of neurons and presumably glia cells, which - together with a concomitant depression of spontaneous brain electrical activity and accompanying cerebral blood flow (CBF) changes - propagates across the cerebral grey matter at a low rate of 2-8 mm/min (Leão, 1944; Somjen, 2001). Cellular depolarization appears on local field potential (LFP) or electrocorticogram (EcoG) recordings filtered in DC mode as a transient negative shift of the DC potential trace, while the concomitant depression of neuronal activity is visualized as a transient local depression of the ECoG.

Since the first observation made by Leão (Leão, 1944), the pathophysiological role of SD has been recognized in several neurological diseases. SD corresponds to migraine aura and likely contributes to headache evolution (Ayata, 2010), whereas accumulating clinical and experimental evidence suggest that SD contributes to secondary injury following both ischemic and hemorrhagic stroke (Helbok et al., 2017; Mun-Bryce et al., 2001; Pinczolits et al., 2017; Woitzik et al., 2013), traumatic head injury (TBI) (Hinzman et al., 2014; Toth et al., 2016) and subarachnoid hemorrhage (SAH) (Dreier et al., 2009; Hamming et al., 2016).

\subsection{Neurophysiological aspects of SD}

\subsubsection{SD elicitation and evolution}

In the healthy mammalian brain, extracellular $\mathrm{K}^{+}$concentration is kept close to $3-4 \mathrm{mM}$, independent of fluctuations in blood serum levels (Somjen, 1979), but local changes in extracellular $\mathrm{K}^{+}$levels do occur following neuronal activity. In the injured brain, the concentration of $\mathrm{K}^{+}(10-15 \mathrm{mM})$ sufficient to induce SD is presumably determined by the balance between $\mathrm{K}^{+}$efflux and the efficacy of $\mathrm{K}^{+}$clearance (Spong et al., 2016). When a critical threshold of both $\mathrm{K}^{+}$levels is reached, the self-propagating SD cycle takes off and invades neighboring tissue (Grafstein, 1956) (Matsuura \& Bureš, 1971; Y. T. Tang et al., 2014). The mass cellular depolarization reflects then a near-complete breakdown of neuronal transmembrane potential (Somjen, 2001). More specifically, at any point of the tissue involved in $\mathrm{SD}$, the influx of $\mathrm{Na}^{+}$leads the depolarization, causing a reduction of extracellular $\mathrm{Na}^{+}$ concentration from 140-150 to 50-70 mM, accompanied by a sudden extracellular surge of $\mathrm{K}^{+}$ from 2.7-3.5 to $30-60 \mathrm{mM}$, a concurrent decrease of extracellular $\mathrm{Ca}^{2+}$ levels from 1-1.5 to 0.2- $0.8 \mathrm{mM}$ and that of $\mathrm{Cl}^{-}$from 130 to $74 \mathrm{mM}$ (Hansen \& Zeuthen, 1981; Pietrobon \& Moskowitz, 2014). Intracellular ion concentrations obviously change in the opposite direction (Fig. 1.). 


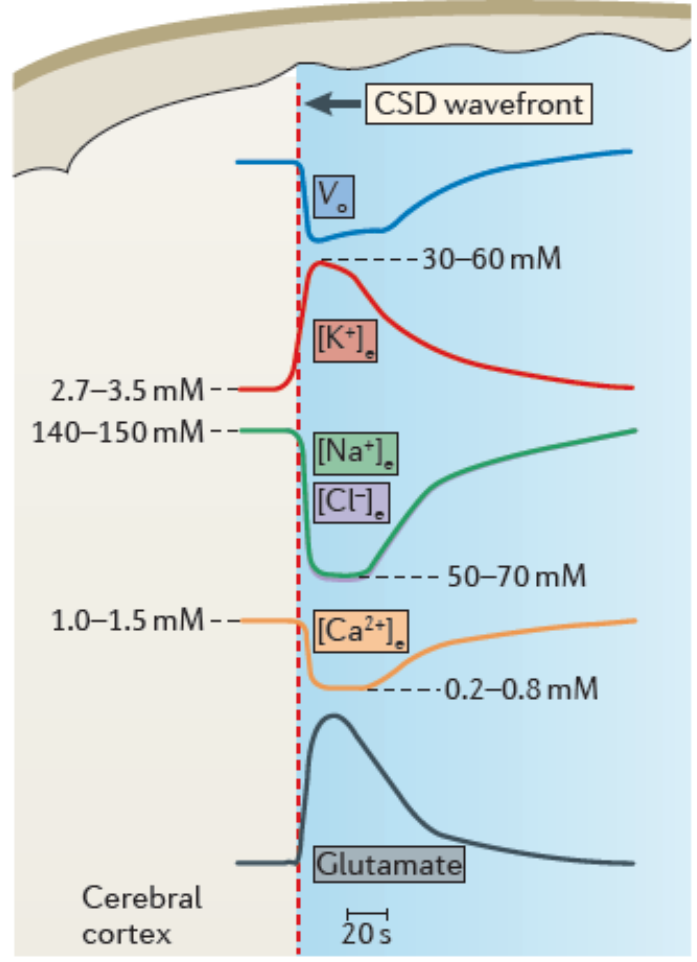

Figure 1. Changes in the extracellular concentrations of $\mathrm{K}^{+}\left(\left[\mathrm{K}^{+}\right]_{\mathrm{e}}\right), \mathrm{Na}^{+}\left(\left[\mathrm{Na}^{+}\right]_{\mathrm{e}}\right), \mathrm{Cl}^{-}\left(\left[\mathrm{Cl}^{-}\right]_{\mathrm{e}}\right), \mathrm{Ca}^{2+}\left(\left[\mathrm{Ca}^{2+}\right]_{\mathrm{e}}\right)$ and glutamate during spreading depolarization. The rapid tissue depolarization during the early phase of spreading depolarization (SD) is accompanied by a rapid $\left[\mathrm{K}^{+}\right]_{\mathrm{e}}$ increase to 30-60 $\mathrm{mM}$ (from a baseline value of 2.7-3.5 $\mathrm{mM}$ ), a $\left[\mathrm{Na}^{+}\right]_{\mathrm{e}}$ and $\left[\mathrm{Cl}^{-}\right]_{\mathrm{e}}$ decrease to $50-70 \mathrm{mM}$ (from $140-150 \mathrm{mM})$ and a $\left[\mathrm{Ca}^{2+}\right]_{\mathrm{e}}$ decrease to $0.2-0.8 \mathrm{mM}$ (from 1.0-1.5 mM). SD and the associated ionic changes are shown to propagate across the cerebral cortex from right to left; the red dashed line represents the SD wave front. SD is labeled as CSD (i.e.: cortical spreading depression). Source: (Pietrobon \& Moskowitz, 2014).

The channels mediating $\mathrm{K}^{+}$efflux during depolarization are still to be explored, but it is reasonable to suggest that voltage-gated potassium $(\mathrm{Kv})$ channels must be involved, as the wide spectrum $\mathrm{K}^{+}$channel blocker tetraethylammonium and 4-aminopyridine partially limited $\mathrm{K}^{+}$ efflux during SD (Aitken et al., 1991; Somjen, 2001). Recent evidence indicates that largeconductance $\mathrm{Ca}^{2+}$-activated $\mathrm{K}^{+}(\mathrm{BK})$ channels contribute to the $\mathrm{K}^{+}$surge with $\mathrm{SD}$ (Menyhart et al., 2018), and it is suspected that during ischemia or anoxia, massive nonselective $\mathrm{Na}^{+}\left(\mathrm{Ca}^{2+}\right) / \mathrm{K}^{+}$conductance take place with $\mathrm{SD}$, via a yet unidentified channel (Czeh et al., 1992; Gagolewicz, 2017). Finally, in case of acute brain injury, the reduced availability of ATP may open ATP-sensitive $\mathrm{K}^{+}$channels in an attempt to hyperpolarize neurons via $\mathrm{K}^{+}$efflux (Sun \& $\mathrm{Hu}, 2010$ ), contributing to the extracellular $\mathrm{K}^{+}$accumulation (Somjen, 2001).

The limited availability of ATP reduces the efficiency of neuronal $\mathrm{Na}^{+} / \mathrm{K}^{+}$-ATPase and thus $\mathrm{K}^{+}$ reuptake (Hajek et al., 1996). In addition, extracellular levels of $\mathrm{K}^{+}$typical of the ischemic penumbra were shown also to decrease $\mathrm{Na}^{+} / \mathrm{K}^{+}$-ATPase activity by $50 \%$ (Major et al., 2017), therefore $\mathrm{K}^{+}$itself may amplify its extracellular accumulation. The central role of $\mathrm{Na}^{+} / \mathrm{K}^{+}$-ATPase in SD initiation is clearly supported by the fact that tissue exposure to ouabain, a $\mathrm{Na}^{+} / \mathrm{K}^{+}$-ATPase inhibitor, readily produces SD (Balestrino et al., 1999). Ultimately, the excess $\mathrm{K}^{+}$in the extracellular space amounts to a depolarizing stimulus that, by the gradual shift in membrane potential, opens voltage-gated $\mathrm{Na}^{+}$channels to give way to $\mathrm{Na}^{+}$influx. The 
experimental elicitation of $\mathrm{SD}$ with high concentration $\mathrm{KCl}$ or electrical stimulation takes advantage of the artificial elevation of extracellular $\mathrm{K}^{+}$as well.

$\mathrm{Ca}^{2+}$ influx with SD may take place through P/Q type voltage-gated calcium (Cav) channels, which was tested by the application of gabapentine, an anticonvulsant agent, with P/Q type Cav channel inhibitory potential. Gabapentin infusion effectively suppressed SD susceptibility in the intact rat cortex (Hoffmann et al., 2010).

In addition to the ion dislocations, interstitial glutamate concentration also increases (Fig. 1.) from 3-3.5 to 10-11 $\mu \mathrm{M}$ with SD induced by $\mathrm{KCl}$ in healthy rat cortex (Hinzman et al., 2015), or well over $100 \mu \mathrm{M}$ during anoxia (Satoh et al., 1999). The SD-related glutamate efflux may be mediated by intracellular $\mathrm{Ca}^{2+}$ accumulation through P/Q type Cav channels (Ayata et al., 2000; Pietrobon \& Moskowitz, 2014) or presynaptic N-methyl-D-aspartate (NMDA) receptordependent vesicular exocytosis (Zhou et al., 2013). Further on, SD has been found to open neuronal pannexin-1 channels (Karatas et al., 2013) that may also mediate the release of glutamate to some extent (Cervetto et al., 2013; Di Cesare Mannelli et al., 2015). Glutamate may also originate from astrocytes through pathological processes, including the possibility that increased extracellular $\mathrm{K}^{+}$concentration reverses excitatory amino acid transporters (EAATs) (Harada et al., 2015; Malarkey \& Parpura, 2008; Nicholls \& Attwell, 1990).

Glutamate accumulation may overstimulate NMDA receptors, deepening depolarization, and contributing to SD propagation by further increasing $\mathrm{K}^{+}$and glutamate release (Harreveld, 1959). Besides post-synaptic NMDA receptors, there is evidence for the role of $\alpha$-amino-3hydroxy-5-methyl-4-isoxazole propionate (AMPA) receptors (Costa et al., 2013) and extrasynaptic NMDA receptors (Hardingham \& Bading, 2010) as well. The involvement of the NMDA receptor in the sustenance of SD is underscored by influential clinical studies disclosing that the administration of ketamine, an NMDA receptor antagonist, to patients of acute brain injury inhibits SD occurrence effectively (Carlson et al., 2018; Sakowitz et al., 2009). It must be noted, however, that SD evolving in response to anoxia cannot be blocked by NMDA receptor antagonism, suggesting the negligible involvement of NMDA receptors in SD propagation under severe metabolic stress (Pietrobon \& Moskowitz, 2014).

The volume of the extracellular space also adjusts the concentration of substances present in the extracellular fluid. Water passively follows the fluxes of $\mathrm{Na}^{+}$and especially $\mathrm{Cl}^{-}$via $\mathrm{Cl}^{-}$-coupled transporters (Steffensen et al., 2015) to cause swelling of dendrites (termed “dendritic beading") and astrocytes (Risher et al., 2012), with a concomitant shrinkage of the 
extracellular space. This may increase the concentrations of extracellular $\mathrm{K}^{+}$and glutamate even further (Hansen \& Olsen, 1980; Phillips \& Nicholson, 1979).

All these results testify that once SD has been elicited, the levels of $\mathrm{K}^{+}$and glutamate, which facilitate SD, progressively rise. The propagation of SD, therefore, is thought to be selfsustained, advocated by the volume transmission of high concentration $\mathrm{K}^{+}$and glutamate generated by SD itself.

\subsubsection{Recovery from SD}

SD is a transient event - although its duration depends on the metabolic conditions of the tissue. The repolarization phase of SD, or recovery of the tissue from SD is mediated by neuronal $\mathrm{Na}^{+} / \mathrm{K}^{+}$-ATPase to serve $\mathrm{K}^{+}$reuptake (Major et al., 2017). Importantly, surplus $\mathrm{K}^{+}$is also removed effectively by astrocytes, utilizing various mechanisms including, for instance, astrocytic $\mathrm{Na}^{+} / \mathrm{K}^{+}$-ATPase, and $\mathrm{K}^{+}$siphoning via inwardly rectifying $\mathrm{K}^{+}$channels (Kir 4.1) or water flux mediated through aquaporin-4 channels (Leis et al., 2005) (Yao et al., 2015). Similar to $\mathrm{K}^{+}$clearance, glutamate buffering is an equally important factor in SD evolution.

Glutamate clearance is mainly mediated by excitatory amino acid transporters 1 and 2 (EAAT1 and EAAT2), which are ion- and voltage- dependent and as such the predisposing glutamate reuptake is highly susceptible to changes in the ionic composition of the cellular environment and transmembrane potential. Among EAAT subtypes, the glial specific EAAT2 appears to be responsible for more than $90 \%$ of glutamate reuptake in the forebrain (Rimmele \& Rosenberg, 2016). EAAT2 is not only co-localized with $\mathrm{Na}^{+} / \mathrm{K}^{+}$-ATPase, but is also highly dependent on the ion gradient it generates (Cholet et al., 2002), thus it is of no surprise that, EAAT2-mediated glutamate clearance was hampered in the absence of the $\alpha 2$ subunit of the astrocytic $\mathrm{Na}^{+} / \mathrm{K}^{+}$ ATPase and was associated with the facilitation of SD initiation (Capuani et al., 2016). Moreover, mice carrying the genetic knock-in of an astrocytic $\mathrm{Na}^{+} / \mathrm{K}^{+}$ATPase $\alpha 2$ subunit loss-of-function mutation - as it occurs in familial hemiplegic migraine type 2 (FHM2) patients - were prone to decreased SD threshold and increased rate of SD propagation (Leo et al., 2011). Conversely, ceftriaxone, one of the $\beta$-lactam antibiotics, stimulated the expression of EAAT2 in astrocytes and concurrently raised SD threshold in the FHM2 mutant mice, while inhibition of EAAT2 in wild type mice lowered SD threshold (Capuani et al., 2016). These results collectively suggest that EAAT2 - in tight coupling with $\mathrm{Na}^{+} / \mathrm{K}^{+}$ATPase - is essential for glutamate clearance by astrocytes with SD. 


\subsubsection{Cerebral blood flow response to spreading depolarization}

$\mathrm{SD}$ is coupled by typical changes in $\mathrm{CBF}$, mediated by the various vasoactive agents released during cellular depolarization(Ayata \& Lauritzen, 2015). In the rat - and most probably in humans - the physiological pattern of the SD-associated CBF response includes four sequential components: (i) an initial, brief hypoperfusion; (ii) a marked, transient peak hyperemia; (iii) a less obvious late hyperemia; and (iv) a sustained hypoperfusion also known as spreading or post-SD oligemia (Ayata \& Lauritzen, 2015). The occurrence, duration and magnitude of these elements is variable, with the peak hyperemic component being the most conspicuous and most extensively studied. The share of each element in the CBF response is prone to the level of baseline cerebral perfusion and the metabolic state of the tissue (Ayata \& Lauritzen, 2015; Dreier, 2011; Pietrobon \& Moskowitz, 2014).

Under physiological conditions (i.e. normal cerebral perfusion), the rise of extracellular $\mathrm{K}^{+}$at the wave-front of an SD episode is the main vasoactive agent responsible for initial hypoperfusion (Menyhart et al., 2018). $\mathrm{K}^{+}$is thought to be directly depolarizing vascular smooth muscle cells, leading to vasoconstriction (Ayata \& Lauritzen, 2015), with astrocytic $\mathrm{K}^{+}$-release likely contributing to perivascular $\mathrm{K}^{+}$accumulation (Filosa et al., 2006). Hypoperfusion is then transformed into peak hyperemia which, even though being the most conspicuous and well-studied part of SD related CBF response, is incompletely understood. Nitric oxide (NO), a potent vasodilator, is released abundantly during SD (Obrenovitch et al., 2002), with concomitant upregulation of neuronal nitric oxide synthase (nNOS) (Ayata \& Lauritzen, 2015; Busija et al., 2008). Thus, NO has been in the center of investigations, with highly conflicting results. As such, various pharmacological inhibitions of NO synthesis in rabbits and cats led to reduced peak hyperemia, which could not be reproduced in rats (Ayata \& Lauritzen, 2015; Busija et al., 2008). In physiological neurovascular coupling, NO is increasingly regarded as a permissive factor rather than the primary vasodilator. The direct contribution of NO to hyperemia persists only for the initial few seconds after the onset of neuronal activation, and becomes negligible under longer-lasting activation (Cauli \& Hamel, 2010). This is underscored by the observation, that inhibition of nNOS increases initial hypoperfusion with SD, rather than attenuating peak hyperemia (Ayata \& Lauritzen, 2015; Busija et al., 2008). Thus, NO may have a permissive role in the SD coupled CBF responses, counteracting vasoconstrictors $\left(\mathrm{K}^{+}\right.$and 20-Hydroxyeicosatetraenoic acid (20-HETE)) and potentiating other vasodilators, like prostanoids (especially prostaglandin $\mathrm{E}_{2}\left(\mathrm{PGE}_{2}\right)$ ), calcitonin-gene related peptide, serotonin, reactive oxygen species and lactate (Ayata \& 
Lauritzen, 2015; Busija et al., 2008). Finally, peak hyperemia is followed by long-lasting (20$60 \mathrm{~min}$ ) oligemia, believed to be mediated by vasoconstrictive arachidonic acid metabolites 20 HETE and prostaglandin $\mathrm{F}_{2 \alpha}\left(\mathrm{PGF}_{2 \alpha}\right)$ (Ayata \& Lauritzen, 2015).

As discussed, prostanoids are emerging as important mediators of the SD related CBF response. They are potent vasoactive metabolites of versatile effects, with acknowledged role in physiological neurovascular coupling. They are derived of arachidonic acid by two rate limiting enzymes: the constitutively expressed - astrocytic - cyclooxygenase-1 (COX-1) and the inducible - neuronal - cyclooxygenase-2 (COX-2). Spreading depolarization coincides with a considerable accumulation of arachidonic acid in the cortex (Lauritzen et al., 1990), and a significant elevation of prostanoid concentration (e.g. $\mathrm{PGF}_{2 \alpha}$ and $\mathrm{PGE}_{2}$ ) in the cerebrospinal fluid (Masaaki Shibata et al., 1992). Arachidonic acid metabolites released due to SD were found to be vasoconstrictive: First, the non-selective inhibition of COX enzymes (i.e. application of indomethacin) caused pial vasodilation with SD, and diminished vasoconstriction underlying post-SD oligemia (Masaaki Shibata et al., 1992). Nonetheless, the selective effect of COX-1 or COX-2 products on the SD-associated CBF response has not been revealed, although the potential involvement of COX-2 is conceivable, because COX-2 mRNA and protein were found upregulated in cortical neurons in association with SD (Miettinen et al., 1997). Second, synthesis of the vasoconstrictive 20-HETE by the P450 epoxygenase enzyme located in vascular smooth muscle cells was demonstrated in response to SD, and the pharmacological blockade of its synthesis ameliorated post-SD oligemia (Fordsmann et al., 2013). In physiological neurovascular coupling however, $\mathrm{PGE}_{2}$ was shown to cause vasodilation by binding to its receptors (EP2 and EP4 receptors) located in the vascular wall in the brain (Cauli \& Hamel, 2010; Lacroix et al., 2015; Myren et al., 2010).

In ischemic settings, with decreasing residual blood flow approaching the ischemic core, the $\mathrm{CBF}$ response to $\mathrm{SD}$ is increasingly dominated by vasoconstrictive elements, leading to diminished hyperemia and more prevalent hypoemia (Feuerstein et al., 2016; Hoffmann \& Ayata, 2013; Menyhart et al., 2015; Woitzik et al., 2013) (Fig. 2.). In the most severe form, the hypoemic element completely outweighs hyperemia, and the CBF response to SD turns into spreading ischemia(Dreier, 2011). This atypical SD-associated CBF variation in the injured brain aggravates metabolic supply-demand mismatch in the tissue, and can delay recovery from SD thereby increasing the risk of irreversible depolarization, neuronal cell death and the expansion of the ischemic core. 


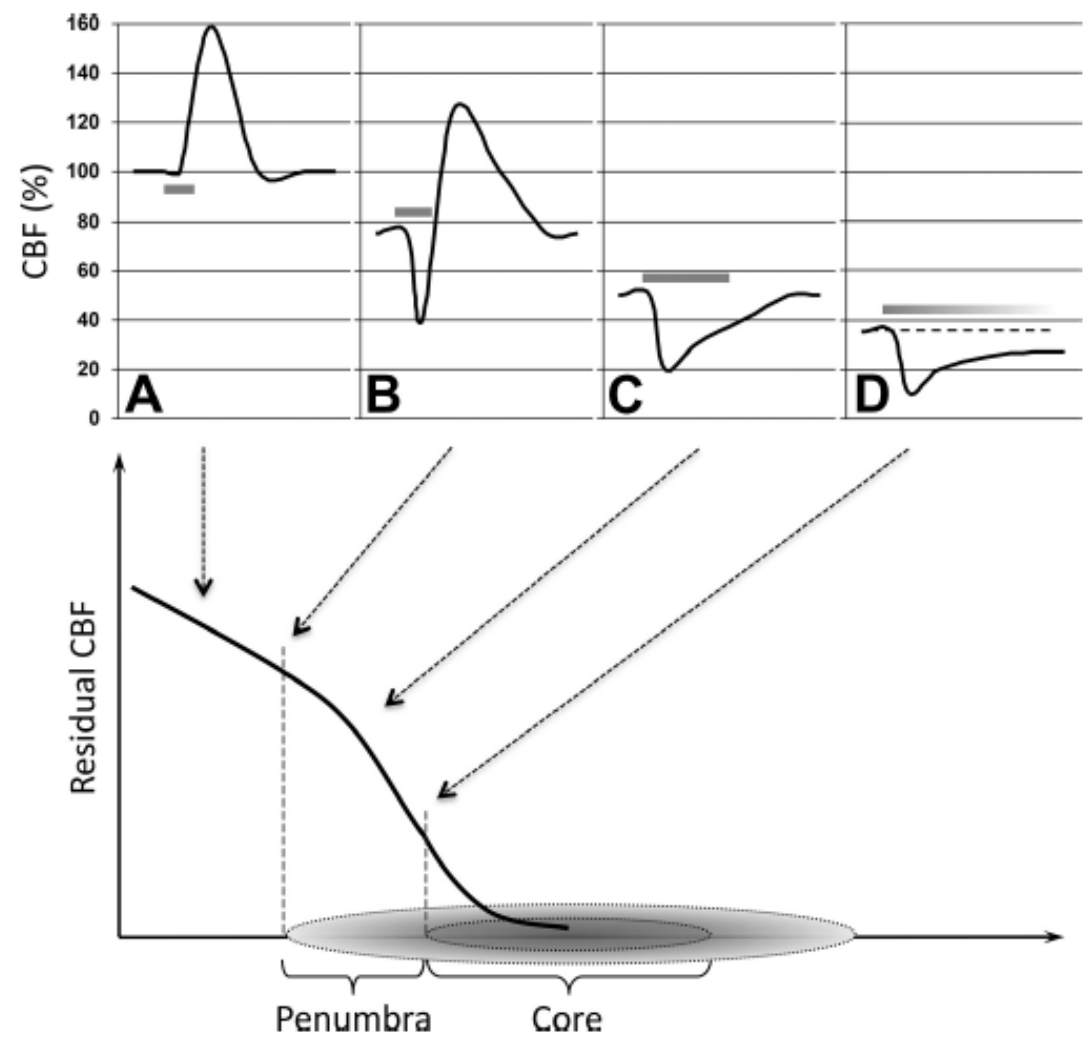

Figure 2. The CBF response to SD in focal cerebral ischemia. A hypothetical diagram shows the transformation of the CBF response to SD (top graphs) against the increasing perfusion deficit (bottom graph). In non-ischemic tissue, SD (a horizontal bar of a length representing SD duration) evokes a predominantly hyperemic response $(A)$, whereas in mildly ischemic penumbra, a biphasic CBF response is observed $(B)$. In the more seriously ischemic penumbra, the CBF response is transient hypoperfusion (i.e. spreading ischemia) $(C)$. At the severely ischemic core-penumbra junction $(D)$, SD becomes terminal, and there is no recovery from the associated hypoperfusion. Source: (Ayata \& Lauritzen, 2015).

\subsection{Secondary injury mediated by SD}

Typically, recurrent SD waves with long cumulative duration may increase neuronal injury (Pinczolits et al., 2017; Woitzik et al., 2013) (Hartings et al., 2017; Nakamura et al., 2010) by (i) NMDA glutamate receptor over-activation that induces $\mathrm{Ca}^{2+}$ overload and neuronal excitotoxicity (Hinzman et al., 2015); (ii) the insufficiency of the CBF response to replenish ATP necessary for the uptake of $\mathrm{K}^{+}$by the $\mathrm{Na}^{+} / \mathrm{K}^{+}$ATPase (Hartings et al., 2017); or (iii) causing persistent tissue acidosis, particularly in aged animals (Menyhart et al., 2017).

The coincidence between the severity of ischemic damage and SD occurrence was corroborated by the linear correlation between the total depolarization time or SD frequency with infarct maturation in rodent focal cerebral ischemia models (Back et al., 1996; Back et al., 1994; Dijkhuizen et al., 1999; K. Takano et al., 1996). Causality was confirmed, when SDs elicited experimentally distant to ischemic foci were shown to propagate to penumbra-like tissue and increase the size of the ischemic infarct (Busch et al., 1996). 


\subsection{Susceptibility of the nervous tissue to SD}

Taken that SD emerges as a potent mechanism of secondary brain injury in patients, it is of great interest to explore which conditions favor the occurrence of SD.

Experimentally, SDs can be triggered in the intact cortex in various ways: chemically (topical or intraparenchymal application of $\mathrm{KCl}$, NMDA agonists, $\mathrm{Na}^{+} / \mathrm{K}^{+}$ATPase blockers) or electrically (epi-or subdural repetitive or anodal electrical stimulation) (Ayata, 2009; Pietrobon \& Moskowitz, 2014). SD susceptibility for evoked SDs may be quantified by implementing cathodal stimulation with direct current and measuring the electrical charge (by using Coulomb's law) necessary for SD evolution (Leo et al., 2011) or as a function of the time required to trigger SD with high-frequency tetanic stimulation or by stepwise raising the amplitude of the stimulus. In contrast, SDs are readily provoked by cerebral injuries (ischemia, cerebral contusion or hemorrhage), in which case SDs develop spontaneously and unpredictably. In focal cerebral ischemia, repetitive SDs are thought to arise from the border of the penumbra and the ischemic core (Bere et al., 2014b; Kao et al., 2014), probably initiated by hypotensive or hypoxic transients (von Bornstadt et al., 2015). Further, the flow threshold was estimated as the lower range of the brain's autoregulatory capacity in global ischemia (Bere et al., 2014a) or defined accurately as being around $40 \mathrm{ml} / \mathrm{g} / \mathrm{min}$ in focal ischemia (Takeda et al., 2011). Yet, the ultimate, direct cause of SD elicitation is the critical, elevated concentration of extracellular $\mathrm{K}^{+}$and/or glutamate (Somjen, 2001), both of which accumulate in the ischemic brain due to increased release from neural elements and altered clearance by astrocytes (Leis et al., 2005).

In addition to calculating the strength of the minimum stimulus to trigger SD as described above, various parameters can be taken to express SD susceptibility, such as (i) the latency of SD occurrence with respect to the stimulus, (ii) the frequency of recurrent SD events, (iii) the rate of SD propagation, (iv) the distance SD covers over its course of propagation, and (v) the duration of ECoG depression. Some of these parameters concern SD initiation (i.e. latency with respect to igniting stimulus), others essentially characterize propagation (i.e. rate and distance covered, duration of ECoG depression), or represent a mixture of both initiation and propagation (i.e. frequency of recurrent SDs). The capability of the nervous tissue to recover from SD may be a relevant factor to consider as well, since delayed repolarization may postpone the occurrence of subsequent SD events, and is expected to reduce SD frequency. The network of astrocytes is thought to contribute to this process. In addition, the buffering capacity of 
astrocytes may also modulate the delay necessary to reach the neurochemical threshold of SD elicitation.

In migraine with aura, the progressive accumulation of $\mathrm{K}^{+}$to high concentration as it takes place in injured tissue is unlikely, therefore an increased sensitivity or hyperexcitability of neurons may stand in the center of SD elicitation (Vinogradova, 2018). In addition, hyperexcitability may be relevant for ischemia-related SDs as well, since theoretically such a condition may predict SD occurrence at a lower threshold. Neuronal excitability is partly regulated by neuronal voltage-dependent $\mathrm{Na}^{+}$(Nav), Kv, and Cav channels (Misonou, 2010). Structural variations, modulation by secondary messengers and genetic dysfunction of these channels might influence SD threshold, the latter being implicated, for example, in FHM type 1 (FHM1) and 3 (FHM3) (Dichgans et al., 2005; van den Maagdenberg et al., 2004). As such, FHM3 has been linked to a mutation of the $\alpha 1$ subunit of Nav 1.1 (encoded by the SNCA1A gene), which elicits neuronal hyperexcitability and might decrease SD threshold (Dichgans et al., 2005). Neuronal hyperexcitability, on the other hand, may be prevented by the activation of various Kv channels, like Kv 1.1, Kv1.2 (Hille, 1992) (G. Chen et al., 2005) and Kv7.2 (KCNQ2) ion channel (Wulff \& Zhorov, 2008), probably via membrane hyperpolarization (Wu et al., 2003).

Neuronal excitability may be modulated by Cav channels as well. FHM1 is characterized by a mutation in the CACNA1A gene encoding the P/Q type $\mathrm{Ca}^{2+}$ channel (van den Maagdenberg et al., 2004), increasing its opening probability at presynaptic terminals (Tottene et al., 2009). Taken together, these experimental data suggest, that voltage-gated cation channels tuning neuronal excitability also contribute to setting the threshold of SD elicitation.

\subsection{Age as a risk factor for neurological diseases in which SD is relevant}

In neurological disorders implicating SD (i.e. migraine with aura, or acute brain injury including $\mathrm{TBI}, \mathrm{SAH}$, and malignant ischemic stroke), age is known as an independent risk factor for the incidence and prevalence of the disorders. For example, the age-dependent prevalence of migraine has been shown to be bimodal, as migraine incidence peaks at the age of 19 and 48 years in men, and at the age of 25 and 50 in women (Victor et al., 2010). Among the general population, TBI has a peak incidence during childhood (falls), adolescence (motor-vehicle accidents) and geriatric age (falls) (Bruns \& Hauser, 2003). Further, SAH is one of the most common types of stroke in young adults, and younger age is an established risk factor for secondary lesion progression in SAH, caused by proximal large artery vasospasm (Charpentier et al., 1999; Rabb et al., 1994) and delayed cerebral ischemia (Crobeddu et al., 2012; de Rooij 
et al., 2013; Magge et al., 2010). Finally, aging significantly predicts poor patient outcomes after ischemic stroke (R. L. Chen et al., 2010; Liu \& McCullough, 2012). The incidence and poor outcome of stroke steeply rises with age (R. L. Chen et al., 2010). In this context, the impact of age on stroke pathophysiology has been the target of intensive research in order to understand the reason for the increased susceptibility of the aged brain to stroke-related injury, yet the potential contribution of SD has remained largely unexplored.

\section{Goals}

SD has been recognized to contribute to migraine with aura and secondary injury following acute brain injuries (Sections 1.1. and 1.2.). The peak prevalence of migraine and traumatic brain injury, for instance, concerns young adulthood (Section 1.4.). Finally, the CBF response to SD is compromised by old age, but less is known about the impact of age over adulthood. Accordingly, our goals were:

I. to determine the innate susceptibility of the cerebral cortex to SD, under non-ischemic and ischemic condition, over the age range from adolescence to young adulthood in rats (7 to 30 weeks) (Sengupta, 2013);

II. to investigate the possible link between the threshold of SD elicitation and the histological organization of the cortex at this age;

III. to explore, whether the CBF response to SD is subject to any age-related modification over the age range investigated;

IV. to identify specific frequency bands of the electrocorticogram that may be selectively affected by SD or age.

SD related CBF changes are the cornerstone of SD related secondary injury. Since prostanoids have been strongly implicated in physiological neurovascular coupling, in a separate study we aimed:

I. to explore the role of vasodilator prostanoids on the SD related CBF response. 


\section{Materials and methods}

\subsection{Surgical procedures}

The experimental procedures were approved by the National Food Chain Safety and Animal Health Directorate of Csongrád county, Hungary. The procedures were performed according to the guidelines of the Scientific Committee of Animal Experimentation of the Hungarian Academy of Sciences (updated Law and Regulations on Animal Protection: 40/2013. (II. 14.) Gov. of Hungary), following the EU Directive 2010/ 63/EU on the protection of animals used for scientific purposes and reported in compliance with the ARRIVE guidelines. For the examination of the age-dependence of SD threshold, young and middle-aged male Sprague-Dawley rats were used $(n=38$, Table 1). Additional, young, adult, male SpragueDawley rats were treated with pharmacological agents to study the regulation of the CBF response to $\mathrm{SD}(\mathrm{n}=60$, Table 2). The animals were purchased from the Charles River Laboratories, Hungary, were group-housed under a normal 12/12 h light/dark cycle, and had free access to food and drinking water.

\begin{tabular}{|ccccc|}
$\begin{array}{c}\text { Age of rats } \\
\text { included in the } \\
\text { study (weeks) }\end{array}$ & Body weight $(\mathrm{g})$ & $\mathrm{n}$ & $\begin{array}{c}\text { Age groups } \\
\text { defined for } \\
\text { data analysis }\end{array}$ & $\mathrm{n}$ \\
\hline 7 & $276 \pm 14$ & 5 & & \\
\hline 8 & $332 \pm 11$ & 4 & $7-10$ weeks & 21 \\
\hline 9 & $348 \pm 11$ & 5 & & \\
10 & $377 \pm 10$ & 7 & 6 & 12 \\
12 & $403 \pm 19$ & 6 & $12-16$ weeks & 5 \\
\hline 16 & $465 \pm 12$ & 6 & 30 weeks & 5 \\
\hline 30 & $608 \pm 37$ & 5 & &
\end{tabular}

Table 1. Composition of experimental age groups in the threshold determination study. (Data are given as mean \pm stdev.)

On the day of experiments, the animals were anesthetized with $1.5-2 \%$ isoflurane in $\mathrm{N}_{2} \mathrm{O}: \mathrm{O}_{2}$ (70\%:30\%) and were allowed to breathe spontaneously throughout the experiment. Body temperature was maintained at $37^{\circ} \mathrm{C}$ with a servo-regulated heating pad. Atropine $(0.1 \%$, $0.05 \mathrm{ml}$ ) was administered intramuscularly shortly before surgical procedures to avoid the production of airway mucus. A catheter was inserted into the right femoral artery to monitor the mean arterial blood pressure (MABP) continuously and the arterial blood gas levels before the elicitation of the first SD and before termination of the experiment (i.e., anesthetic overdose). Next, a midline incision was made in the neck and both common carotid arteries were delicately separated from the surrounding tissue, including the vagal nerves. Lidocaine (1\%) was administered topically before opening each tissue layer. A silicone coated fishing line 
used as occluder was looped around each artery for later induction of cerebral ischemia. Rats were transferred to a stereotactic frame and fixed in prone position.

In the study to determine SD threshold, two cranial windows ("'3 x $3 \mathrm{~mm}$ ) $1 \mathrm{~mm}$ apart were prepared over the right parietal cortex. The bone was carefully thinned using a dental drill (Technobox, Bien Air 810) and gently peeled away to reveal the dura surface. In the caudal window, a small hole was carefully cut on the dura for the positioning of an intracortical microelectrode and an adjacent laser-Doppler probe positioned above the cortical surface (see 3.2 below). SDs were triggered at a regular pattern with electric stimulation (see 3.4 below).

Similarly, in the pharmacological study two craniotomies $(5 \mathrm{~mm}$ lateral from midline, $-3 \mathrm{~mm}$ and $-7 \mathrm{~mm}$ caudal from bregma) were prepared over the right parietal cortex using a dental drill. A doughnut shape ring of acrylic dental cement was built around the rostral craniotomy for the latter topical application of drugs to the cortical surface. The dura in each craniotomy was carefully dissected, and the craniotomies were regularly irrigated with artificial cerebrospinal fluid (aCSF; mM concentrations: $126.6 \mathrm{NaCl}, 3 \mathrm{KCl}, 1.5 \mathrm{CaCl} 2,1.2 \mathrm{MgCl}, 24.5 \mathrm{NaHCO} 3,6.7$ urea, 3.7 glucose bubbled with $95 \% \mathrm{O} 2$ and $5 \% \mathrm{CO} 2$ to achieve a constant $\mathrm{pH}$ of 7.4). The rostral window was used to confirm SD evolution with electrophysiological recording, and to assess local changes of CBF (see 3.2 below). SDs were elicited in the caudal craniotomy chemically (see 3.5 below).

\subsection{Recording of electrophysiological parameters and cerebral blood flow}

For the concomitant recording of ECoG and slow cortical or direct current (DC) potential, a glass capillary electrode $(20 \mu \mathrm{m}$ outside tip diameter) filled with physiological saline was inserted 800-1000 $\mu \mathrm{m}$ deep into the cerebral cortex at the recording window. $\mathrm{An} \mathrm{Ag} / \mathrm{AgCl}$ reference electrode was implanted under the skin of the animal's neck. DC potential and ECoG were recorded via a high input impedance pre-amplifier (NL102G, NeuroLog System, Digitimer Ltd., United Kingdom), connected to a differential amplifier (NL106, NeuroLog System, Digitimer Ltd., United Kingdom) with associated filter and conditioner systems (NL125, NL530, NeuroLog System, Digitimer Ltd., United Kingdom). Potential line frequency noise $(50 \mathrm{~Hz})$ was removed by a high quality noise eliminator (HumBug, Quest Scientific Instruments Inc., Canada) without any signal attenuation. The resulting signal was digitalized by an analog/digital (A/D) converter (MP150, Biopac Systems Inc., USA) and continuously acquired at a sampling frequency of $1 \mathrm{kHz}$ using the software ACQKNOWLEDGE 4.2.0 (Biopac Systems Inc., USA). 
SD-associated changes in local CBF were recorded using laser-Doppler flowmetry (LDF). A laser Doppler needle probe (Probe 403 connected to PeriFlux 5000; Perimed AB, Sweden) was positioned right above the cortical surface at the penetration site of the glass capillary electrode with a micromanipulator, avoiding any large pial vessels. The signal was digitized and acquired, together with the DC potential and ECoG, essentially as described above.

\subsection{Experimental protocol to determine SD threshold}

Following a baseline period of over an hour, persistent incomplete global forebrain ischemia was induced by occluding both common carotid arteries permanently (" 2 -vessel occlusion," 2VO): occluders were pulled on until resistance was felt and then secured in place. Successful ischemia induction was confirmed by a sharp drop of the LDF signal. A concentric bipolar needle electrode (tip size: $40 \mu \mathrm{m}$, Neuronelektród Kft., Hungary) was placed upon the dura in the rostral window for SD elicitation. It was connected to an opto-coupled stimulus isolator with a constant current output (NL 800, Digitimer Ltd., United Kingdom), a pulse generator (NL301), a with-delay panel (NL405), and a pulse buffer (NL510), which enabled the adjustment of amplitude and duration of the stimuli at will. Stimulation was implemented with a single, cathodal, and rectangular pulse. The charge delivered was quantified as $Q[\mu C]=$ $I[m A] \times t[m s]$, and it was raised stepwise with an interstimulus interval of 2 min until SD was observed. Whenever necessary, the position of the needle electrode was adjusted to optimize the contact between the electrode tip and the tissue. Successful elicitation of SD was confirmed by a negative DC- shift of an amplitude greater than $5 \mathrm{mV}$ acquired by the recording electrode or by obvious ECoG depression.

Three SDs were elicited during the baseline period, at an inter-SD interval of at least $20 \mathrm{~min}$. Additional three SDs were generated during ischemia, the first triggered 20-min after ischemia onset or after the spontaneous occurrence of an SD event associated with ischemia induction (Fig. 3.). 


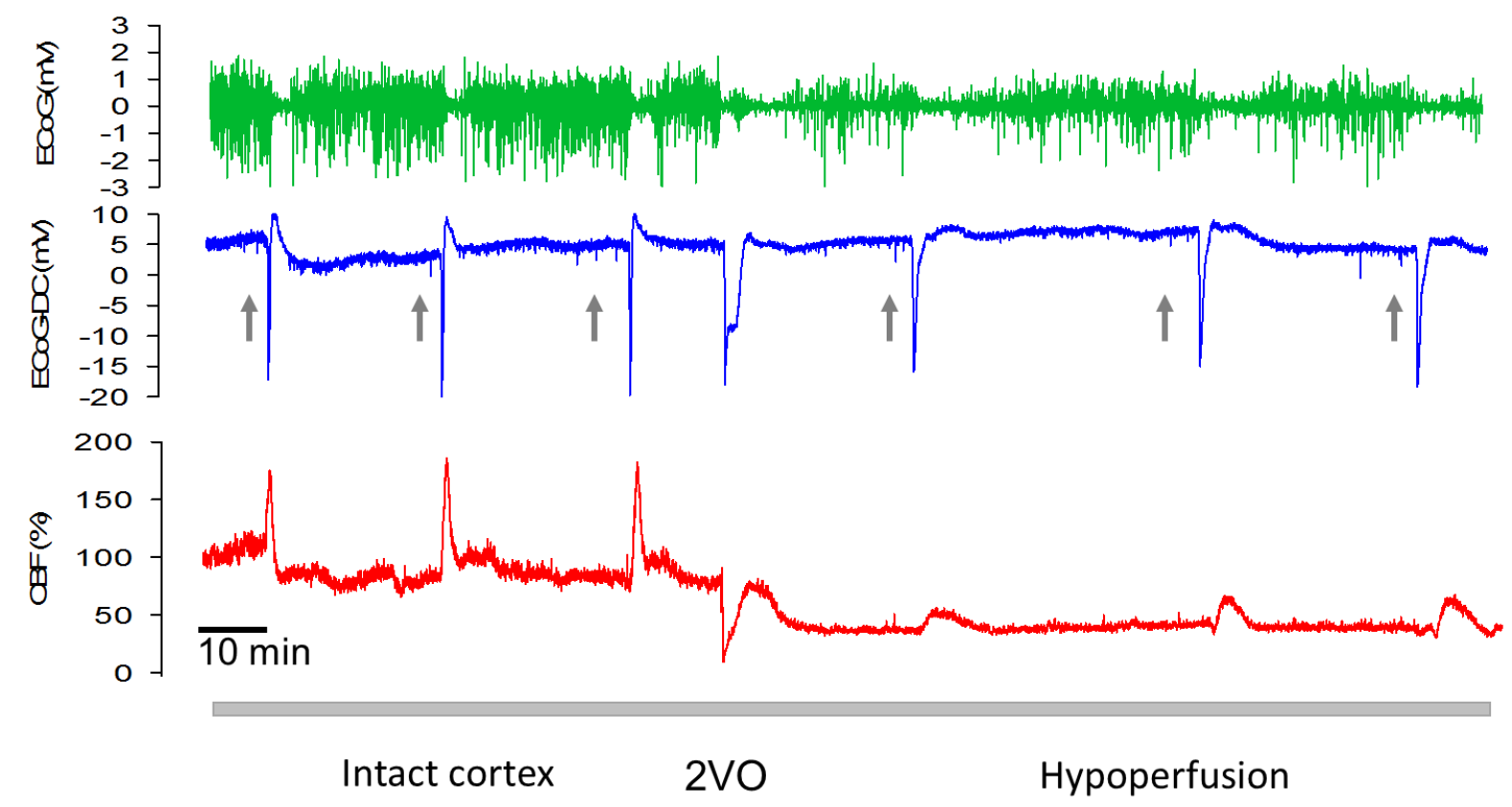

Figure 3. Representative, synchronous traces of the ECoG, DC potential, and CBF, acquired from an 8-week-old animal from the threshold determination study. Depression of the ECoG synchronous with a transient, negative shift of the DC potential and associated functional hyperemia together indicate SD occurrence. The label $2 \mathrm{VO}$ (bilateral common carotid artery occlusion, "2-vessel occlusion") designates ischemia induction. Short vertical lines on the DC potential trace show the delivery of current to evoke SD (upward gray arrows). Ischemia induction was immediately followed by the occurrence of a single spontaneous SD in this experiment.

\subsection{Experimental protocol for pharmacological investigation}

Drug solutions or vehicle of equal volume (1.5\% DMSO in $10 \mathrm{ml}$ aCSF) were superfused on the cortical surface free of dura in the rostral cranial window after taking DC potential and CBF baseline for 5 min under aCSF. Drug concentrations were carefully selected based on dose response curves, selectivity and efficacy reported previously (Lacroix et al., 2015; Myren et al., 2010; Niwa et al., 2000; Niwa et al., 2001). The following drugs were applied topically: the selective COX-2 inhibitor NS-398 (100 $\mu$ M; Cayman) (Niwa et al., 2000), the selective COX-

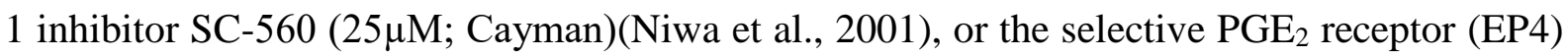
antagonist L161,982 (1 $\mu \mathrm{M}$; Sigma) (Hall et al., 2014; Lacroix et al., 2015). The pharmacological treatment was initiated $40 \mathrm{~min}$ prior to ischemia induction or the elicitation of the first SD event (i.e. sham-operated group) (Niwa et al., 2000), and incubation persisted till the end of the experimental protocol.

Following the 40-min incubation period, persistent incomplete global forebrain ischemia was induced in half of the animals by $2 \mathrm{VO}$ (see 3.4 above). As control for ischemia, the remaining animals were used as a sham-operated group (SHAM), in which the occluders were not pulled but left in place. 
Forty minutes after the start of drug incubation, 4 SDs with an inter-SD interval of 15 minutes were triggered by placing a $1 \mathrm{M} \mathrm{KCl-soaked} \mathrm{cotton} \mathrm{ball} \mathrm{on} \mathrm{the} \mathrm{exposed} \mathrm{cortical} \mathrm{surface} \mathrm{in} \mathrm{the}$ caudal cranial window. The cotton ball was removed and the caudal cranial window rinsed with aCSF immediately after each successful SD elicitation. Experiments were terminated by the overdose of the anesthetic agent, 20 min after triggering the last SD. The combination of pharmacological treatment and ischemia induction resulted in 8 experimental groups (Table 2.). Higher prevalence of severe ischemia, terminal depolarization and mortality in the ischemic animals treated with L161,982 was noted, requiring higher numbers in that experimental group.

\begin{tabular}{c|c|cc}
\hline $\begin{array}{c}\text { Experimental } \\
\text { group }\end{array}$ & $\begin{array}{c}\text { Pharmacological } \\
\text { treatment }\end{array}$ & $\begin{array}{c}\text { Cerebral blood flow } \\
\text { (Intact or Ischemia) }\end{array}$ & $\mathrm{n}$ \\
\hline 1 & $\begin{array}{c}\text { Vehicle } \\
(1.5 \% \text { DMSO in } \\
\text { aCSF) }\end{array}$ & Intact & 8 \\
\hline 2 & $\begin{array}{c}\text { Ischemia } \\
\text { (COX } \mu \text { M NS-398 inhibition) }\end{array}$ & Intact & 7 \\
\hline 3 & Ischemia & 6 \\
\hline 4 & $\begin{array}{c}25 \mu M \text { SC-560 } \\
\text { (COX-1 inhibition) }\end{array}$ & Intact & 7 \\
\hline 5 & $\begin{array}{c}1 \mu \text { Ischemia } \\
\text { (EP4 receptor } \\
\text { antagonism) }\end{array}$ & Intact & 6 \\
\hline 7 & Ischemia & 12 \\
\hline 8 & &
\end{tabular}

Table 2. Composition of experimental groups in the pharmacological study.

\subsection{Histology}

In order to assess whether dendritic spine density changes with advancing age are related to SD susceptibility, additional 8-week-old $(n=5)$ and 30-week-old $(n=5)$ rats were deeply anesthetized with an overdose of chloral hydrate (i.p.). The animals were transcardially perfused with ice cold saline, decapitated, and the brains quickly removed. The forebrains were cut in the coronal plane into two pieces of equal size and immersed in Golgi-COX solution mixed of the following stock solutions: (i) 5\% potassium dichromate (Molar Chemicals Kft., Hungary), (ii) $5 \%$ potassium chromate (Molar Chemicals Kft, Hungary), and (iii) $5 \%$ mercuric chloride (VWR International, LLC)(Glaser \& Van der Loos, 1981). The tissue blocks were incubated for 10 days at room temperature, the solution being refreshed every 2-3 days. The brain samples were then transferred to $30 \%$ sucrose to be stored for at least 10 days at $4{ }^{\circ} \mathrm{C}$. Coronal slices of $200 \mu \mathrm{m}$ were cut with a vibrating microtome, mounted on gelatin-coated microscopic slides, and stored overnight in a dark humidity chamber. Finally, the staining was 
developed with 30\% ammonium hydroxide followed by Carestream Kodak Fixer (Sigma). Sections were then dehydrated and cover- slipped with Eukitt (O. Kindler, Germany).

The stained brain slices were examined by a light microscope (Nikon Eclipse E600). Fifteensixteen cortical pyramidal neurons in layer 3 were studied in samples of each animal. Dendritic spine density of second- or third-order dendrites of the proximal apical dendrite was analyzed on a 50- $\mu \mathrm{m}$-long segment. Serial z-stack images of selected dendritic segments were created using a SPOT RT Slider digital camera (1600 x 1200 dpi in 8 bits) connected to the light microscope (Nikon Eclipse E600) and a computer equipped with an image processing software (Image Pro Plus 4.5; Media Cybernetics, Bethesda). Dendritic spines were counted by four independent investigators blinded to the experimental groups, using the software IMAGE J (v1.44, National Institute of Health, Bethesda).

\subsection{Data analysis}

All variables (i.e., DC potential, ECoG, LDF signal and MABP) were simultaneously acquired, displayed live, and stored using a personal computer equipped with a dedicated software (AcqKnowledge 4.2 for MP 150, Biopac Systems, Inc., USA). Data analysis was assisted by the inbuilt tools of the software, or was transferred into a MATLAB environment (MathWorks Inc., USA).

Raw LDF recordings were down-sampled to $1 \mathrm{~Hz}$ and then expressed relative to baseline by using the average CBF value preceding the first evoked SD (100\%) and the recorded biological zero obtained after terminating the experiment $(0 \%)$ as reference points. The following elements of the SD-related CBF response were characterized: amplitude and duration of early hypoperfusion, amplitude and duration of hyperemia, magnitude of hyperemia (area under the curve), and the amplitude of post-SD oligemia.

The first SD was evaluated separately from subsequent, recurrent SDs, because of the obvious differences in the kinetics of the SD-associated CBF response. Data of the 3 recurrent SDs were averaged for each animal, thus a single value per animal of each read-out was taken for statistical analysis.

ECoG spectral power analysis was applied for individual frequency bands as described previously(Menyhart et al., 2015). The parameters obtained were the following: (i) level of baseline (the mean spectral power for the 60s interval preceding the onset of an SD); (ii) level of depression (the arithmetic mean between the endpoints of the downward and upward 
segments); (iii) duration of depression; and (iv) level of recovery (the mean spectral power for the 60-s interval following recovery). Along with the 30-week-old group $(n=5)$, six of the youngest animals (7/8 weeks) with clean ECoG trace (i.e., minimal noise) were selected for the analysis.

In order to determine dendritic spine density, the spine count of 15-16 dendritic segments assessed in each animal was averaged, and, thereby, a single value was taken for each rat for further statistical analysis.

Data are given as mean \pm stdev. The software SPSS (IBM SPSS Statistics for Windows, Version 22.0, IBM Corp.) was used for statistical analysis. A one-way analysis of variance (ANOVA) model was used for the evaluation of dendritic spine density, the SD-associated CBF response, baseline ECoG power, and the duration of SD-related ECoG depression. A two-way ANOVA paradigm was applied for the evaluation of data concerning the electric threshold of SD elicitation (factors: age and ischemia) and ECoG power during the SD-related depression (factors: age and ischemia). A repeated measures ANOVA (factor: age) was used for the analysis of resting $\mathrm{CBF}$, taken at selected time points over the course of experiments, and ECoG power variations over the course of experiments. Wherever appropriate, a Fisher post hoc test was used to reveal the differences between the experimental groups. Pearson two-tailed correlation analysis was carried out to test potential association between data sets. Levels of significance were defined as $\mathrm{p}<0.05^{*}$ and $\mathrm{p}<0.01^{* *}$.

\section{Results}

\subsection{Investigation of SD susceptibility}

Preliminary screening of the acquired data sets revealed no age-related difference between the 7-10-week-old animals and the 12-16-week-old animals (inserts in Figure 5(a)), therefore, three age groups were created by uniting the 7-10-week-old and the 12-16-week-old populations (Table 1).

MABP during baseline was statistically not different across groups $(98 \pm 4,95 \pm 3$, and $107 \pm$ $10 \mathrm{mmHg}, 7-10$-week-old, 12-16-week-old, and 30-week-old, respectively). Ischemia elevated MABP slightly but not significantly, without any notable impact of age $(105 \pm 5,96 \pm 4$, and $114 \pm 2$ mmHg, 7-10-week-old, 12-16-week-old, and 30-week-old, respectively). Arterial blood gases were typically within the physiological range, with no significant effect of ischemia or age on either $\mathrm{pCO}_{2}$ or $\mathrm{pH}$. Arterial blood glucose concentration taken during baseline and late ischemia was similar (e.g., $9.23 \pm 1.7$ vs. $9.20 \pm 1.2 \mathrm{mM}$, ischemia vs. baseline in 7-10- 
week-old) but significantly increased with advancing age (e.g., $11.95 \pm 1.6$ vs. $10.08 \pm 1.0$ vs. $9.2 \pm 1.2 \mathrm{mM}, 30$-week-old vs. 12-16-week-old vs. 7-10-week-old during baseline).

SDs occurred reliably upon electric stimulation of the cortex. In a number of experiments (25 of 38), a single, spontaneous SD evolved immediately after ischemia induction (Fig. 3). The DC potential signature of SDs demonstrated that the amplitude of spontaneous SDs $(23.1 \pm 7.8$ $\mathrm{mV}$ ) was statistically not different from SDs evoked during baseline $(24.5 \pm 3.8 \mathrm{mV})$ or ischemia $(28.8 \pm 5.8 \mathrm{mV})$. However, with spontaneous SDs, the duration at half amplitude of the DC shift was considerably longer - albeit with excessive variation (220.9 $\pm 316.0 \mathrm{~s})$, as compared with SDs evoked during baseline $(19.9 \pm 5.1 \mathrm{~s})$ or ischemia $(80.7 \pm 80.8 \mathrm{~s})$. Such spontaneous events were not distinctively prevalent for any of the age groups, although only 2 of the 25 events were recorded in the 30-week-old group. Spontaneous SDs were not included in further data analysis. The CBF response to SDs evoked was invariably hyperemic in all age groups, under both baseline and ischemia. The CBF response to the first SD in each experiment differed from subsequent SDs in that hyperemia was often preceded by a short drop in CBF, and was always followed by long-lasting oligemia.

\subsubsection{Compensation of cerebral blood flow to ischemia}

In order to estimate how compensation for the reduction of $\mathrm{CBF}$ evolved following ischemia induction, $\mathrm{CBF}$ values were obtained prior to and shortly after ischemia onset, and in between SD events evoked during ischemia (Fig. 4). In the youngest, 7-10-week-old group, CBF dropped to $18 \pm 6 \%$ immediately after $2 \mathrm{VO}$ onset, recovered to $37 \pm 12 \%$ before the elicitation of the first SD under ischemia, and was maintained over 30\% throughout the ischemic period. In contrast, $\mathrm{CBF}$ fell to $11 \pm 6 \%$ after ischemia induction, recovered to $20 \pm 13 \%$ before initiation of the first ischemic SD, and remained at around only $20 \%$ in the 30 -week-old group, implying significantly less efficient compensation in the 30-week-old group with respect to the 7-10week-old group. 


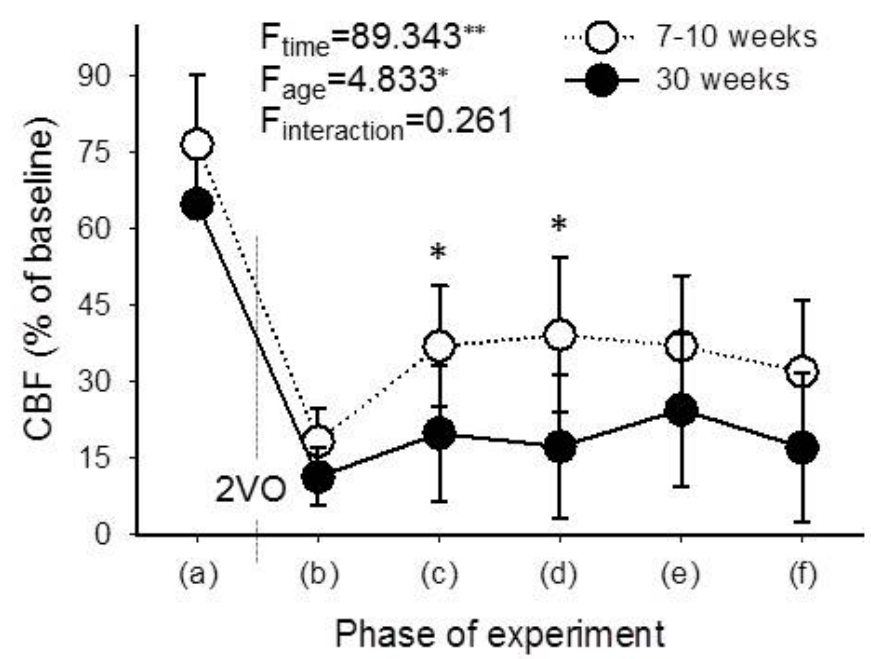

Figure 4. Level of local CBF in the youngest (7-10-week-old) and oldest (30-week-old) age groups, taken at selected time points of the experimental protocol: (a) prior to bilateral common carotid artery occlusion (2VO), after the passage of the last SD elicited during baseline; (b) transient drop immediately after 2VO; (c) shortly before the first ischemic SD; (d) shortly before the second ischemic SD; (e) shortly before the third ischemic SD; (f) late ischemia, after the passage of the last SD elicited during ischemia. Data are presented as mean \pm stdev. A repeated measures analysis of variance (ANOVA) paradigm considering age as a factor was applied for statistical analysis. Level of significance was defined as $\mathrm{p}<0.01 * *$. Post hoc analysis for group comparison at each time point revealed significant difference at (c) and (d). Level of significance was defined as $\mathrm{p}<0.05^{*}$.

\subsubsection{Electric threshold of SD elicitation}

The electric threshold of SD elicitation was expressed as the lowest electric charge sufficient to trigger SD in the cortex (Fig. 5A). We chose to trigger SDs by electric stimulation as opposed to high concentration $\mathrm{K}^{+}$application, because the electric charge delivered can be finely tuned. This offers the accurate estimation of the electric threshold of SD elicitation to uncover any fine variations between age groups of a few weeks difference.

In all age groups, the threshold of SD elicitation was significantly higher during ischemia, as compared with baseline. The initiation of SD required increasingly greater electric charge with older age during both baseline (4743 \pm 1282 vs. $3076 \pm 915$ vs. $1661 \pm 649 \mu \mathrm{C}, 30$-week-old vs. 12 -16-week-old vs. $7-10$-week-old) and ischemia ( $8447 \pm 1763$ vs. $5343 \pm 2170$ vs. $2514 \pm 1032$ $\mu \mathrm{C}, 30$-week-old vs. 12-16-week-old vs. 7-10-week-old). Finally, with advancing age, the threshold during ischemia progressively departed from the threshold determined for the respective baseline: in other words, the difference between ischemia and baseline thresholds was approximately 5 times greater in the 30-week-old as compared with the 7-10-week-old group (threshold difference of $4278 \pm 2352$ vs. $853 \pm 839 \mu \mathrm{C}, 30$-week-old vs. 7 -10-week-old). Next, we set out to determine any potential association between the electric threshold of SD elicitation and CBF, or the cortex' SD-related electrical activity. Lower CBF taken prior SD elicitation predicted higher threshold of SD elicitation $(\mathrm{r}=-0.403 * *)$ (Fig. 5B). The threshold of 
SD elicitation positively correlated with the duration of SD-related depression of the high frequency alpha and beta bands ( $\mathrm{r}=0.373^{* *}$ and $0.478^{* *}$, respectively) (Fig. 5C), but not with the low frequency delta and theta bands ( $r=0.247$ and 0.234 , respectively). No association with any other ECoG variable (i.e. power of baseline, depression or recovery) emerged.
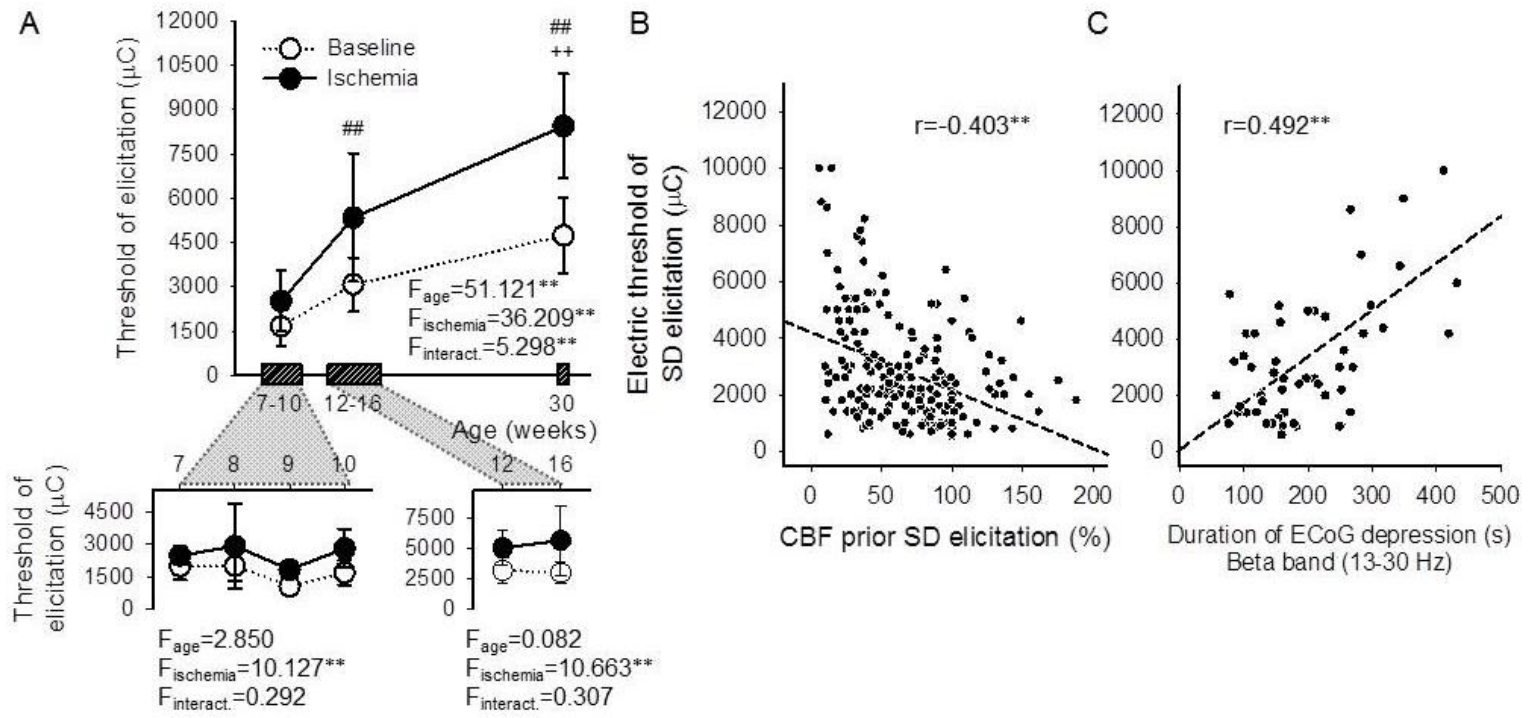

Figure 5. Electric threshold of SD elicitation, and its association with CBF and the SD-related depression of the ECoG. (a) Electric threshold of SD elicitation prior and during incomplete forebrain ischemia (baseline: open symbols, ischemia: closed symbols). The 7-10-week-old animals and 12-16-week-old animals were treated as single groups, because no impact of age was observed on threshold values (see inserts). Data are represented as mean \pm stdev. For the evaluation of statistical significance, a two-way ANOVA paradigm considering age and ischemia as its factors was followed by a Fisher post hoc test for age. Level of significance for the ANOVA was defined as $\mathrm{p}<0.01^{* *}$, and for the post hoc test as $\mathrm{p}<0.01 \#$, vs. $7-10$ week-old and $\mathrm{p}<0.05^{++}$, vs. 12-16 week-old. (b) Negative correlation between the electric threshold of SD elicitation and CBF prior SD evolution; each evoked SD was considered for the analysis. Pearson two-tailed correlation analysis including all age groups as one data pool indicated significant association between the threshold and CBF $\left(\mathrm{p}<0.01^{* *}\right)$. (c) Correlation between the electric threshold of SD elicitation and the duration of SD-related depression on the beta frequency band of the ECoG. Data acquired from animals selected for ECoG spectral analysis are shown. Pearson two-tailed correlation analysis revealed significant association between the data sets $\left(\mathrm{p}<0.01^{* *}\right)$.

\subsubsection{Density of dendritic spines}

We set out to test the hypothesis that the increase in the threshold of SD elicitation due to brain maturation is determined - at least in part - by the dynamic structural development of the cortex, reflected by the morphological plasticity of dendritic spines. The animals admitted to the analysis were naïve (i.e. neither ischemia nor SD was induced prior to sampling), therefore potential dendritic spine density changes due to ischemia or SD propagation were not studied.

Dendritic spines on $2^{\text {nd }}$ or $3^{\text {rd }}$ order dendritic branches of layer 3 pyramidal neuronal apical dendrites were investigated (Fig. 6A). In general, dendritic spines tended to be arranged in clusters rather than individually, and appeared to be larger and more complex in shape in the 30-week-old group than in the 8-week-old group (Fig. 6B). Dendritic spine density 
significantly increased in the 30 -week-old group with respect to the 8 -week-old group $(55 \pm 4$ vs.51 \pm 2 spines / $50 \mu \mathrm{m}$ dendritic segment, 30-week-old vs. 8-week-old) (Fig. 6C).
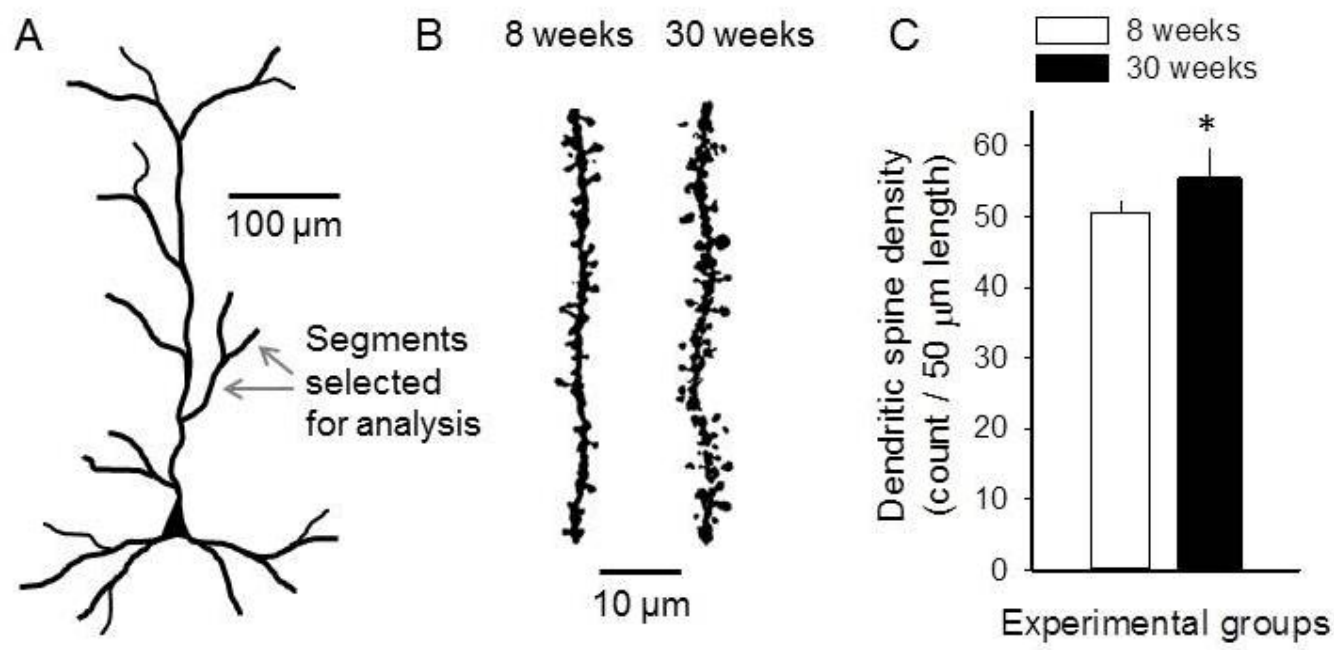

Figure 6. Dendritic spine density on second- or third-order dendritic branches of apical dendrites of layer 3 pyramidal neurons. (a) Schematic drawing of a cortical pyramidal neuron to illustrate the position of dendritic segments investigated. (b) Representative images of 50- $\mu \mathrm{m}$-long segments of second- or third-order dendrites in the 8-week-old and the 30-week-old groups. Images were obtained of original z-stack images by the enhancement of contrast and brightness in order to eliminate background. (c) Dendritic spine density on the selected dendritic segments. Data are given as mean \pm stdev. A one-way ANOVA model was used for statistical analysis $(n=5$ for each group, $\mathrm{F}=6.263, \mathrm{p}<0.05 *)$.

\subsubsection{Features of the cerebral blood flow response associated with SD}

For the evaluation of the SD-associated CBF response, SDs were sorted into three distinct categories: (i) SD1, (ii) subsequent SDs evoked during baseline, and (iii) SDs initiated under ischemia (Fig. 7A). 

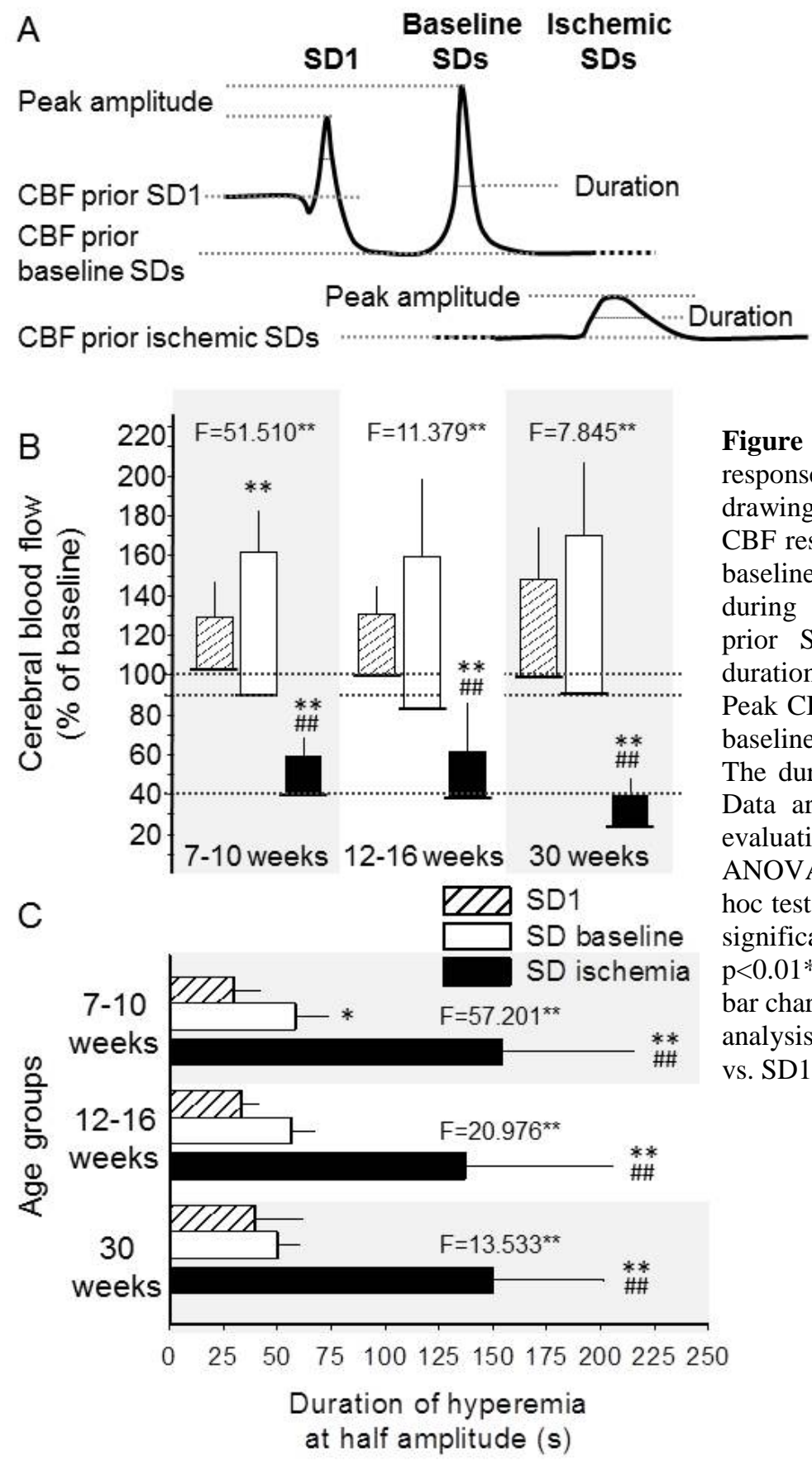

Figure 7. Quantitative analysis of the CBF response associated with SD. (a) Schematic drawings depicting the variables under study: $\mathrm{CBF}$ response to SD1, subsequent SDs during baseline (baseline SDs), and SDs evoked during ischemia (ischemic SDs). CBF level prior SD elicitation, peak amplitude, and duration at half amplitude were evaluated. (b) Peak CBF amplitude (bars) respective to CBF baseline prior SD elicitation (base of bars). (c) The duration of hyperemia at half amplitude. Data are expressed as mean \pm stdev. For the evaluation of statistical significance, a one-way ANOVA paradigm followed by a Fisher post hoc test was used for each age group. Level of significance for the ANOVA was defined as $\mathrm{p}<0.01^{* *}$, and the F-values are given in each bar chart. Level of significance for the post hoc analysis is indicated as $\mathrm{p}<0.05^{*}$ and $\mathrm{p}<0.01^{* *}$ vs. SD1; and $\mathrm{p}<0.01^{\# \#}$ vs. baseline SDs.

As presented in Figure 8B, CBF prior to the onset of baseline SDs was 10-15\% lower than that prior to SD1 in all age groups (e.g. $90 \pm 30$ vs. $102 \pm 12 \%$, baseline SDs vs. SD1 in $7-10$-weekold). The CBF response with SDs under ischemia took off from a markedly lower level $(39 \pm 15$ and 38 $\pm 11 \%, 7-10$-week-old and 12-16-week-old, respectively), especially in the 30-week-old group (23 $\pm 14 \%)$. The SD-associated hyperemia peaked at around $160-170 \%$ in all age groups during baseline (i.e. $163 \pm 45,160 \pm 61$ and $170 \pm 69 \%$, 7-10-week-old, 12-16-week-old and 30week-old, respectively), approximately $20-30 \%$ higher than with SD1 (129 $\pm 23,131 \pm 17$ and $149 \pm 24 \%, 7-10$-week-old, 12-16-week-old and 30-week-old, respectively). During ischemia, the peak amplitude of the CBF response reached a considerably lower level $(59 \pm 23$ and 
$61 \pm 18 \%, \%$, in 7-10-week-old and 12-16-week-old, respectively), with the lowest value

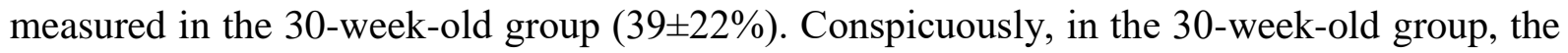
CBF response with ischemic SDs set off from the lowest CBF level, and reached the lowest peak (not even exceeding the resting CBF prior to SD elicitation in the 7-10- or the 12-16-weekold groups) (Fig. 7B), although with no statistically significant reduction with respect to the younger age groups.

Figure 7C shows that the duration of the SD-related CBF response was shortest with SD1 (30 $\pm 12,33 \pm 7$ and $39 \pm 23$ s, 7-10-week-old, 12-16-week-old and 30-week-old, respectively), increased with subsequent SDs elicited during baseline $(58 \pm 14,56 \pm 11$ and $50 \pm 10 \mathrm{~s}, 7-10$-weekold, 12-16-week-old and 30-week-old, respectively), and more than doubled with SDs triggered under ischemia (154 $\pm 61,137 \pm 68$ and $150 \pm 52 \mathrm{~s}, 7$-10-week-old, 12-16-week-old and 30-weekold, respectively). No impact of age on the duration of the SD-associated CBF response was observed.

\subsubsection{Spectral analysis of the distinct ECoG frequency bands}

Baseline ECoG power was assessed at the very beginning of the experiments, prior to any intervention, to test whether anesthesia differentially affects the two age groups. No age-related difference was observed concerning any of the frequency bands studied (Fig. 8A). ECoG power repeatedly sampled prior SD events demonstrated that ischemia itself imposed a considerable power decrease on the overall ECoG, which was particularly prominent after the passage of the first SD evoked under ischemia. This is illustrated in Figure 9B for the theta band.

The novel findings of spectral analysis concerned the SD-related transient depression of the ECoG. Although the power of the four frequency bands during depression was similar under baseline and ischemia (i.e. ischemia exerted no impact), the selectively greater power of the alpha and theta bands in the 30-week-old group as compared with the 7/8-week-old group (e.g. theta band: $0.005 \pm 0.003$ vs. $0.0025 \pm 0.001 \mathrm{~V}^{2}, 30$-week-old vs. $7 / 8$-week-old) indicated incomplete depression of these two bands with the maturation of the brain, under both baseline and ischemic conditions (Fig. 8A). 


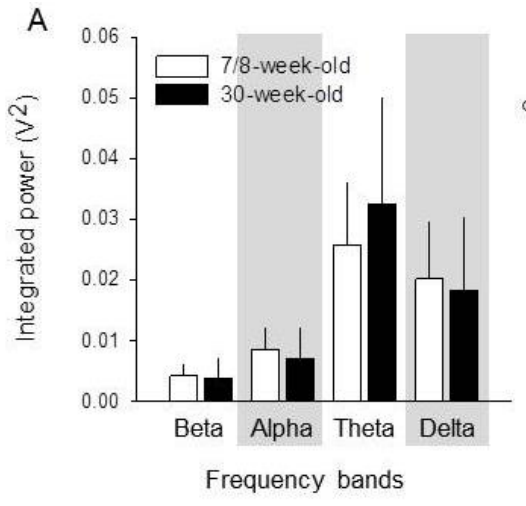

B

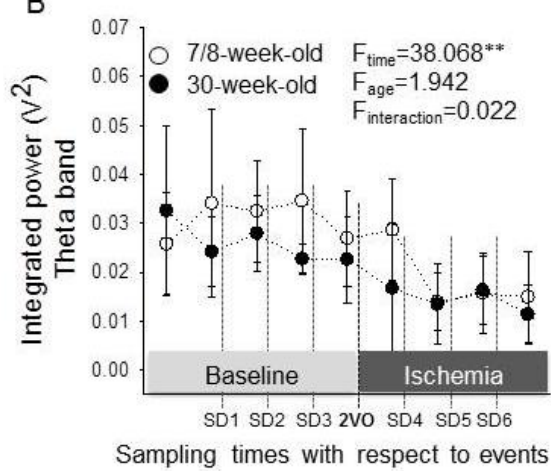

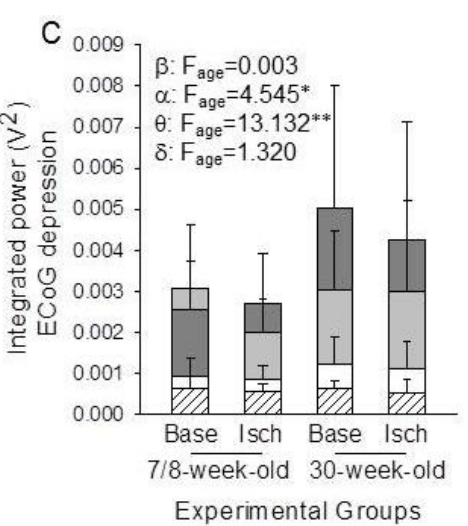

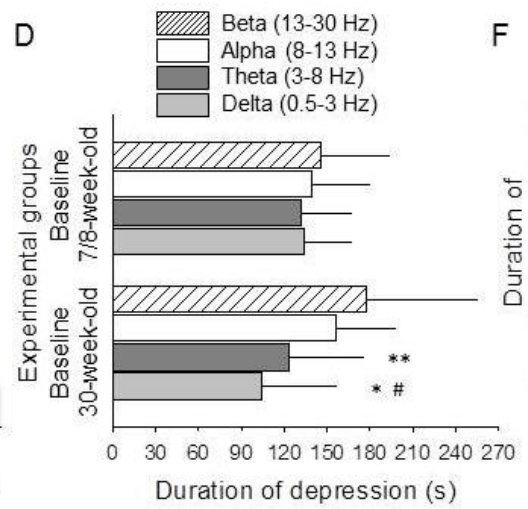

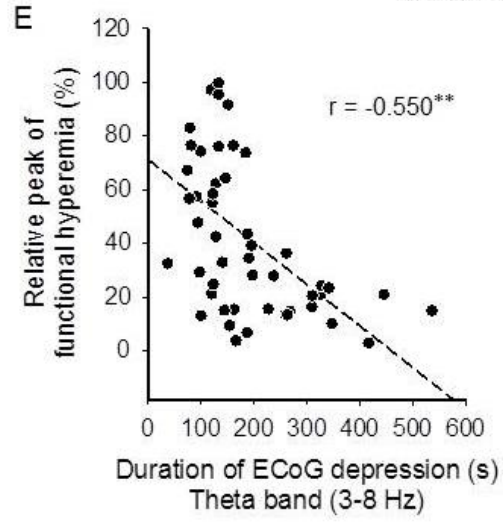

Figure 8. Spectrum analysis of the ECoG for individual frequency bands during SD events. (a) ECoG power of each frequency band, for $60 \mathrm{~s}$ baseline at the very start of the experimental protocol, prior to any intervention. No significant difference between age groups was observed, for any of the frequency bands examined (one-way ANOVA). Note that the weight of low frequency bands in the spectrum is greater than that of higher frequency waves, which mainly reflects an inherent feature of ECoG spectra. Because the ECoG frequency bands represent a power spectrum, their values will vary exponentially, meaning the lower-frequency bands will have exponentially greater values than the higher-frequency bands. (b) Baseline ECoG power of the theta band, assessed repeatedly prior to events, in order to follow the distribution of baseline over the course of the experimental protocol. The first pair of symbols corresponds with theta in Panel A. Repeated measures ANOVA revealed that the ECoG power of baseline decreased, especially during ischemia, irrespective of age. (c) ECoG power of distinct frequency bands, calculated for the period of SD-related ECoG depression. The power of individual frequency bands is represented by bars positioned behind each other. Two-way ANOVA considering age and ischemia as its factors revealed that age significantly elevated the power of the alpha and theta bands selectively, irrespective of ischemia. Level of significance was defined as $\mathrm{p}<0.05^{*}$ and $\mathrm{p}<0.01^{* *}$. (d) Duration of ECoG depression with SDs evoked under baseline condition. The low frequency bands start to recover significantly sooner than the high frequency bands in the 30-week-old group (one-way ANOVA followed by a Fisher post hoc test, $\mathrm{p}<0.05^{\#}$ vs. alpha, $\mathrm{p}<0.05^{*}$ and $\mathrm{p}<0.01^{* *}$ vs. beta). (e) Negative correlation between the duration of the SD-related depression on the theta band of the ECoG, and the relative peak of SD coupled hyperemia. Pearson two-tailed correlation analysis revealed significant association between these data sets $(\mathrm{p}<0.01 * *)$. (f) Positive correlation between the duration of the SD-related depression on the theta band of the ECoG, and the duration of SD coupled hyperemia. Pearson two-tailed correlation analysis indicated significant association between the data sets $(\mathrm{p}<0.01 * *)$.

As expected, ischemia considerably extended the duration of ECoG depression on all four frequency bands (e.g. alpha band in the 7/8-week-old: $271 \pm 126$ vs. $139 \pm 40 \mathrm{~s}$, ischemia vs. baseline). Curiously, during baseline, the lower frequency delta and theta bands started to recover from the SD-related depression sooner than the higher frequency alpha and beta bands in the 30-week-old group, in contrast with the 7/8-week-old group (e.g. duration of theta band: $123 \pm 52$ vs. $132 \pm 34$ s, 30-week-old vs. 7/8-week-old) (Fig. 8B). 
The duration of the SD-related ECoG depression appeared to be associated with the SD-coupled CBF response: the relative peak of SD-associated hyperemia correlated negatively with the duration of ECoG depression on all four frequency bands, as illustrated for the theta band in Figure $8 \mathrm{C}\left(\mathrm{r}=-0.550^{* *}\right)$. At the same time, the longer duration of hyperemia was strongly linked to the longer duration of ECoG depression on all four frequency bands, being most prominent on the delta and theta bands ( $\mathrm{r}=0.733 * *$ and $\mathrm{r}=0.703 * *$, respectively) (Fig. 8D).

\subsection{Pharmacological manipulations to explore potential mediators of the cerebral blood flow response to $\mathrm{SD}$}

\subsubsection{Effect of pharmacological manipulation on systemic physiological variables and baseline CBF levels}

Topical application of drugs was chosen in order to avoid potential systemic side effects. Measured values of MABP and the outcome of blood gas analysis confirmed no difference in the systemic variables assessed in various treatment groups. Specifically, Figure 9A demonstrates that MABP did not change significantly over the experimental protocol, and was similar across experimental groups. Arterial blood gas analysis showed that all investigated variables were within the physiological range at the start of the experiments (i.e. $\mathrm{pO}_{2}: 133 \pm 28$ $\mathrm{mmHg}, \mathrm{pCO}_{2}: 32 \pm 6 \mathrm{mmHg}, \mathrm{pH}: 7.39 \pm 0.02, \mathrm{HCO}_{3}: 19.19 \pm 4.54 \mathrm{mmol} / \mathrm{l}$, glucose: $5.21 \pm 1.71$ $\mathrm{mmol} / \mathrm{l}$ ) and prior to the termination of the experiments (i.e. $\mathrm{pO}_{2}: 125 \pm 29 \mathrm{mmHg}, \mathrm{pCO}_{2}: 38 \pm 11$ mmHg, pH: 7.37 $\pm 0.08, \mathrm{HCO}_{3}: 21.49 \pm 3.44 \mathrm{mmol} / 1$, glucose: $\left.6.11 \pm 1.92 \mathrm{mmol} / \mathrm{l}\right)$. Baseline CBF was obviously reduced significantly due to ischemia induction (from $112 \pm 15$ to $27 \pm 13 \%$ as an average), but the various treatments did not exert any significant impact (Fig. 9B).
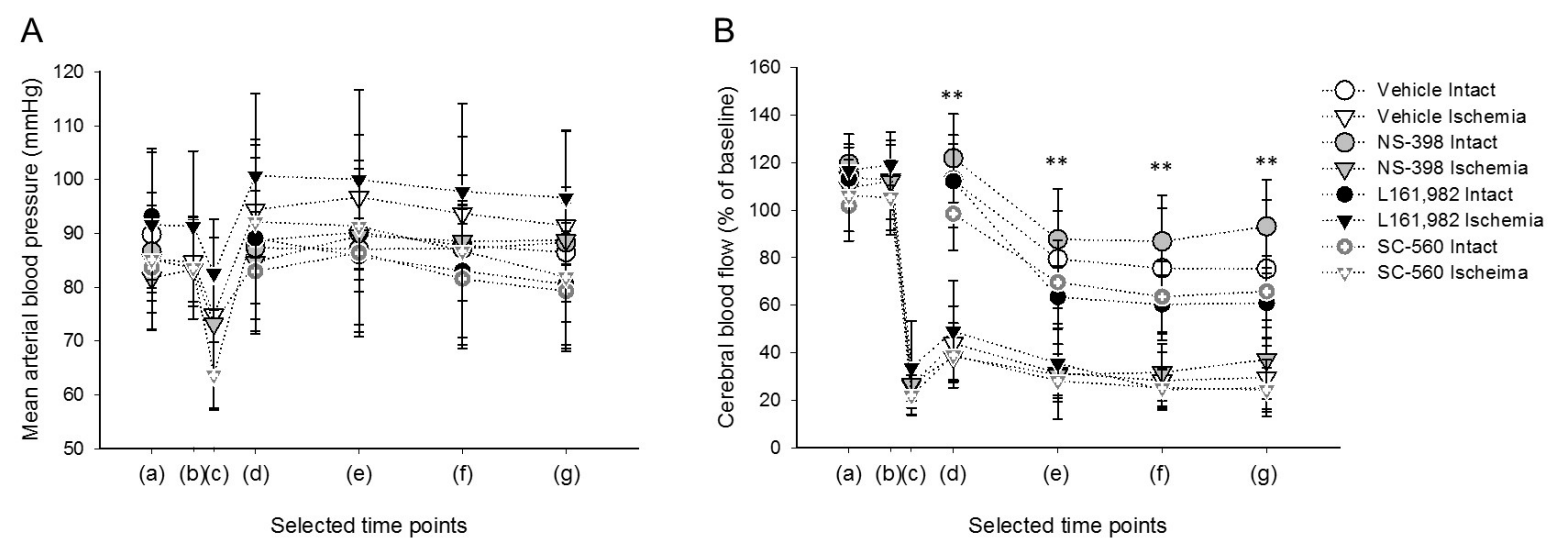

Figure 9. Mean arterial blood pressure (MABP; in A) and drift of local cerebral blood flow (CBF; in B) over the experimental protocol. Selected time points for sampling have been defined as follows: (a) at 35 min after the initiation of pharmacological treatments; (b) immediately before ischemia induction; (c) MABP (A) or CBF (B) minimum after ischemia induction; (d) prior to SD1; (e) prior to SD2; (f) prior to SD3; (g) prior to SD4. Data are given as mean \pm stdev. Statistical analysis relied on a repeated measures paradigm followed by a Fisher post hoc test. Ischemia - but not treatment - had a significant effect on the baseline drift of CBF. Level of significance was determined as $\mathrm{p}<0.01 * *$ vs. respective Intact group. 


\subsubsection{Pharmacological manipulation of SD related cerebral blood flow response}

The impact of pharmacological treatments on the CBF response could be discriminated in the intact - but not in the ischemic - animals. The selective COX enzyme inhibitors NS-398 and SC-560 did not exert any clear influence on the evolution of the SD-related CBF response, as all the examined variables remained unaltered by the treatments. On the other hand, the EP4 receptor blocker L161,982 selectively reduced the relative amplitude of peak hyperemia with the first SD (21 \pm 11 vs. $51 \pm 38 \%$, Intact L161,982 vs. Intact Vehicle), and recurrent SDs (50 \pm 21 vs. $76 \pm 37 \%$, Intact L161,982 vs. Intact Vehicle). In fact, L161,982 lowered the amplitude of the hyperemic element of the CBF response to near the level observed for the ischemic group (first SD: $21 \pm 11$ vs. $15 \pm 8 \%$, L161,982 Intact vs. L161,982 Ischemic), as indicated by the loss of statistically significant difference otherwise obvious between the intact-ischemic group pairs (i.e. Vehicle, NS-398 or SC-560 treated) (Fig. 10B). Further, L161,982 augmented the relative amplitude of post-SD oligemia with the first SD (58 \pm 13 vs. $40 \pm 14 \%$, Intact L161,982 vs. Intact Vehicle) (Figure 10A\&C). Based on these results, the selective EP4 receptor blocker L161,982 appeared to achieve a general loss of a dilatory element prevalent throughout the CBF response, as reflected by a reduction of peak hyperemia with a degree matching the deepening of the postSD oligemia (Fig. 10A). This observation was confirmed by calculating the flow difference between peak hyperemia and the minimum point of post-SD oligemia, providing the exploited range of vasoregulation during the $\mathrm{CBF}$ response, which was unchanged due to treatments in the intact groups ( $87 \pm 32 \mathrm{pp}, \%$ in average). 

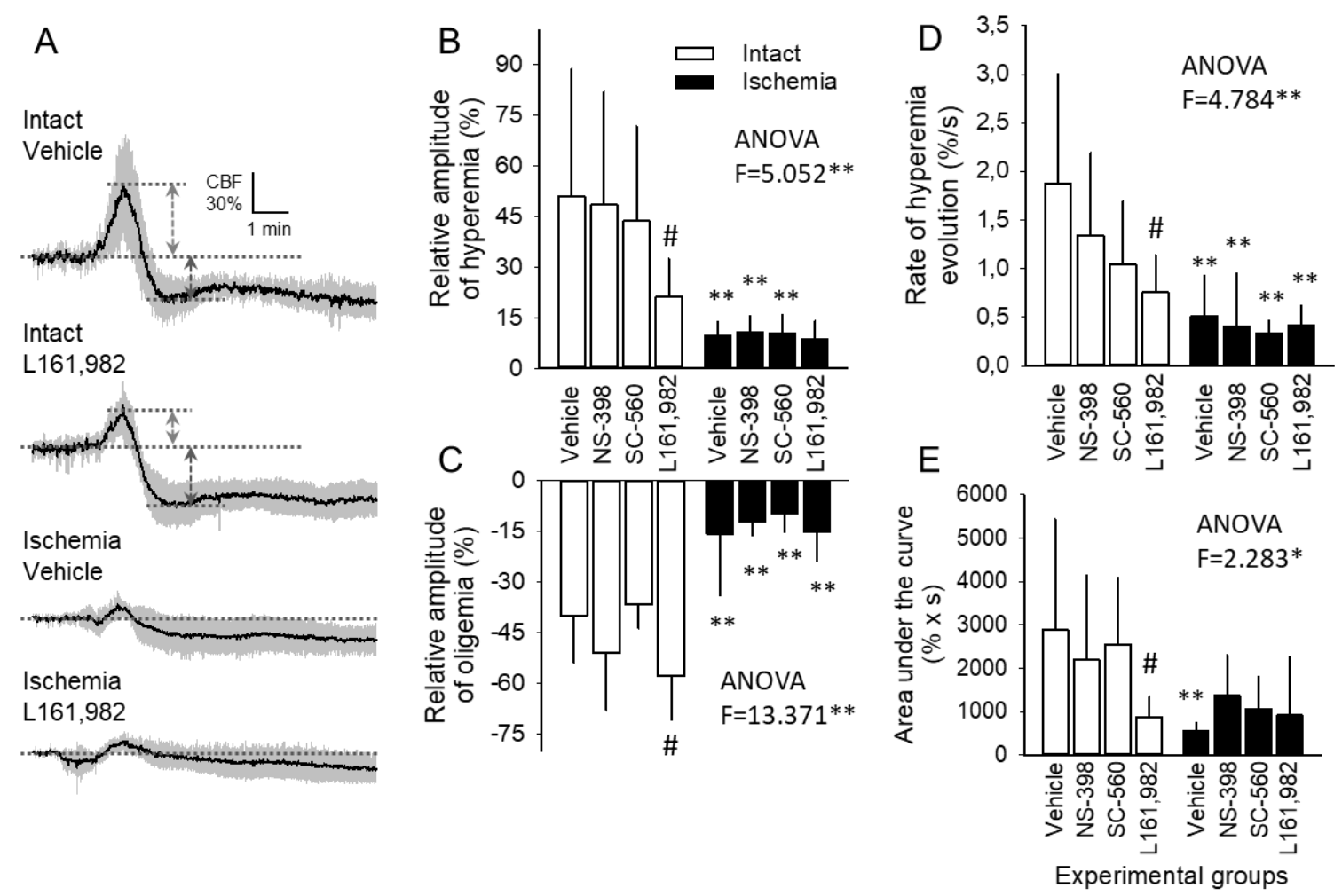

Figure 10. The impact of pharmacological treatments on the kinetics of the spreading depolarization (SD)-related cerebral blood flow (CBF) response. All panels demonstrate data for the first SD. A, CBF traces are mean \pm stdev for each group presented. B, Maximum amplitude of peak hyperemia; C, Minimum amplitude of post-SD oligemia; D, Rate of hyperemia evolution associated with SD; E, Magnitude of hyperemia expressed as area under the curve. Data in Panels B-E are given as mean \pm stdev. Statistical analysis relied on a one-way analysis of variance (ANOVA) model followed by a Fisher post hoc test. Statistical significance was determined as $p^{*}<0.05$ and $\mathrm{p}^{* *<0.01}$ vs. respective Intact, and $\mathrm{p} \#<0.05$ vs. respective Vehicle. Note the selective effect of L161,982 (i.e. EP4 receptor antagonism) under Intact condition.

To further prove the selective effect of L161,982 on the SD-related CBF response, hyperemia evolution significantly decelerated due to L161,982 treatment as demonstrated by its shallower upward slope with the first SD $(0.75 \pm 0.38$ vs. $1.88 \pm 1.13 \% / s$, Intact L161,982 vs. Intact Vehicle) (Fig. 10D), and recurrent SDs (1.05 \pm 0.43 vs. $1.60 \pm 0.70 \% / \mathrm{s}$, Intact L161,982 vs. Intact Vehicle). Finally, the magnitude of hyperemia expressed as the area under the curve was also significantly decreased by L161,982 for the first SD ( $874 \pm 462$ vs. $2885 \pm 2543 \%$ x s, Intact L161,982 vs. Intact Vehicle) (Fig. 10E), and recurrent SDs (5532 \pm 3643 vs. $7448 \pm 2072$ \% x s, Intact L161,982 vs. Intact Vehicle).

The duration of the hyperemic element of the CBF was unchanged by pharmacological treatment in the Intact group, while in the ischemic group it was closely related to DC potential duration. The coincidence between the duration of depolarization and the associated hyperemia was confirmed by a strong, positive correlation $\left(\mathrm{r}=0.825^{* *}\right)$ between these variables, similarly to the correlation of ECoG depression and CBF response duration (Figure 8F). 
Finally, the early hypoperfusion element of the SD-related CBF response did not appear consistently, therefore measured values (i.e. amplitude, duration) could not be analyzed reliably.

Pharmacological manipulation proved to be ineffective on the kinetics of SD in physiological setting. Conversely, the DC potential signature of SD was markedly and selectively elongated by SC-560 and L161,982 but not NS-398 treatment in the Ischemic group.

\section{Discussion}

We investigated the susceptibility of the nervous tissue to SD, focusing on young adults of increasing age.

I. We found, that the electric threshold of SD elicitation in young adult rats significantly increased during early adulthood, while incomplete forebrain ischemia decreased the susceptibility of the cerebral cortex to SD.

II. The increasing threshold of SD elicitation coincided with the increasing density of dendritic spines of cerebrocortical pyramidal neurons.

III. The age-related impairment of CBF compensation examined over an hour following ischemia onset becomes first apparent at 30 weeks of age in the Sprague-Dawley rat. The SD related CBF response was impaired by ischemia, without any detectable impact of age in the age-groups investigated.

IV. The duration of SD-related depression of activity was shorter, specifically on the delta and theta bands. The SD-related depression of the alpha and theta frequency bands were less pronounced in older animals.

In a separate study we investigated the role of vasodilator prostanoids (especially $\mathrm{PGE}_{2}$ ) in the regulation of the SD related $\mathrm{CBF}$ response.

I. The pharmacological antagonism of the $\mathrm{EP}_{4}$ receptor of $\mathrm{PGE}_{2}$ decreased hyperemia and exacerbated post-SD oligemia under physiological circulation, without influencing tissue depolarization. Importantly, pharmacological manipulation had no effect under ischemia.

\subsection{The impact of ischemia on SD elicitation}

Our present data clearly demonstrate, that the electric threshold of SD elicitation markedly increases with ischemia and that the ischemia-related threshold elevation is increasingly more obvious with older age (Fig. 5A). 
The ischemia-related increase of SD threshold may be the result of the metabolic state of the tissue, as indicated by the correspondence between lower CBF taken prior to evoking an SD with a higher electric threshold of SD elicitation (Fig. 5B). The supply of glucose and oxygen are crucial for the proper working of energy-dependent ion pumps, and thus the effective maintenance of resting membrane potential. The first indication that the restricted availability of energy substrates facilitates, while surplus glucose impedes SD was delivered by creating systemic hypo- and hyperglycemia in rats. Hypoglycemia shortened, while hyperglycemia postponed the onset delay of SD in response to hypoxia initiation (Hansen, 1978). Later, hyperglycemia (23-24 mM) was also shown to elevate the electric threshold of SD elicitation, and to reduce the frequency of high $\mathrm{K}^{+}$-induced recurrent SDs in normally-perfused tissue (Hoffmann et al., 2013). Hypoglycemia (2,2 mM), on the other hand, did not alter SD susceptibility, but prolonged the cumulative duration of recurrent SDs elicited in the otherwise intact cortex (Hoffmann et al., 2013). Interestingly, suppression of glycogen, lactate or glucose utilization in the cerebral cortex of mice reduce SD elicitation threshold, suggesting a significant role for astrocyte-neuron lactate shuttle during SD (Kilic et al., 2018). In conclusion, the unlimited supply of circulating plasma glucose may restrict the repeated occurrence of SD in the intact and ischemic cerebral cortex. However, our data on blood glucose level indicated normoglycemic conditions (blood glucose concentration around 9-11 $\mathrm{mM}$ ) in all age groups, and revealed no difference between baseline and late ischemia. Therefore, we suggest that the higher threshold of SD under ischemia was unrelated to blood glucose concentration.

It has been long appreciated that mild tissue acidosis - which typically characterizes ischemic penumbra tissue - suppresses SD- For example, pH 6.67-6.97, achieved by $\mathrm{HCl}$ or $\mathrm{NaOH}$ application, the elevation of $\mathrm{pCO}_{2}$ or withdrawal of bicarbonate in the medium of brain slice preparations inhibited SD initiation and reduced the velocity of SD propagation (Tombaugh \& Somjen, 1996; Tong \& Chesler, 2000). Low pH may restrict SD evolution via NMDA receptor inhibition (C. M. Tang et al., 1990) or by the adjustment of the conductance and gating properties of Kv, Nav, and Cav channels (Tombaugh \& Somjen, 1996). Therefore, we suggest that the ischemia-related fall of tissue $\mathrm{pH}$ could be a key factor in raising the threshold of SD elicitation as observed here. Finally, increased $\mathrm{K}^{+}$conductance and the gradual accumulation of extracellular $\mathrm{K}^{+}$that occur during ischemia generally contribute to membrane hyperpolarization and repolarization thereby depressing neuronal excitability (Shah and Aizeman, 2014). This ionic imbalance may effectively inhibit SD elicitation as well. 


\subsection{The impact of aging on SD elicitation}

The neonatal brain appears to be too immature to sustain experimentally triggered SD. In the intact rat cortex, SD can be first initiated from postnatal days 12-15 (Bures, 1957; Frank Richter et al., 1998; Schade, 1959). The threshold of SD elicitation was thought to decrease until adulthood, and expected to decrease further with aging, theoretically due to the shrinkage of the extracellular space (Somjen, 2001).

However, the latter view on aging appears to be superseded by accumulating experimental evidence. First, the rate of SD propagation was shown to decelerate in the aging rodent brain (Guedes et al., 1996). Also, increasingly higher concentration of $\mathrm{KCl}$ was required to trigger $\mathrm{SD}$ in brain slices obtained from middle-aged rats with respect to young adults (Maslarova et al., 2011), which was later confirmed by our research group in anesthetized rat cerebral cortex (Menyhart et al., 2015; Menyhart et al., 2017). We have previously observed, that the same, incessant, standard trigger $(1 \mathrm{M} \mathrm{KCl})$ produced a lower number of recurrent SDs in the middleaged with respect to the young adult cerebral cortex in anesthetized rats (Farkas et al., 2011).

In further support for the concept that the aged nervous tissue is less able to sustain SD, additional experimental results may be lined up. In the full band ECoG, SD is seen as a transient, spreading depression of activity (Leão, 1944) - unless the ECoG is already isoelectric prior to SD occurrence due to a severe insult (Hartings et al., 2011a). The duration of the ECoG depression in the non-ischemic rat cerebral cortex may last for over $2 \mathrm{~min}$ in the young, but for only half of this time duration in middle-aged animals (Farkas et al., 2011).

In the present study, we investigated the susceptibility changes to $\mathrm{SD}$ in young rats, corresponding to human adolescence and young adulthood (Sengupta, 2013). By using electric stimulation, we have precisely defined and confirmed previous results, that the threshold of SD initiation increases with age, and we have shown that the susceptibility of the cerebral cortex to SD starts to decrease gradually already from adolescence on.

The increased threshold of SD elicitation with advancing age during early adulthood may be linked to structural changes in neuronal networks, which may alter the electrophysiological properties of the nervous tissue. Thus, it is conceivable that the stimulus applied is dissipated in the tissue before SD is ignited. The formation and retraction of dendritic spines that host the post-synaptic element of excitatory synapses dynamically changes with brain maturation (Harris, 1992) and aging (Dickstein et al., 2013) is heavily involved in SD propagation (Herreras, 1993) and is negatively affected by SD (Risher et al., 2010). Therefore we set out to 
determine dendritic spine density in the youngest and oldest animals admitted to our study to contemplate on any potential link between age-related structural changes in the cortex, and the threshold of SD elicitation. Our results exhibited an increased density of dendritic spines on the apical dendrites of cortical layer 3 pyramidal neurons at 30 weeks of age with respect to 8 weeks of age (Fig. 6), proving that the histological organization of the cortex undergoes detectable alterations during the life span investigated in this study.

Even though a direct link between such fine structural changes and the excitability of neurons is challenging to establish, we speculate that the threshold of SD elicitation may increase with age during early adulthood because of the histological (and connected biochemical) maturation or consolidation of cortical connections. Further on, some evidence suggest that the excitability of the aged nervous tissue is lower, because the age-specific increase in the production of reactive oxygen species modifies the operation of the redox-sensitive $\mathrm{K}^{+}$channels (Sesti, 2016) (Fig. 11.B). This process may modulate the oligomer formation, permeation and gating properties of $\mathrm{Kv}$ channels and BK channels (Sesti, 2016), but it remains to be explored whether these changes manifest at the level of SD evolution. 
A

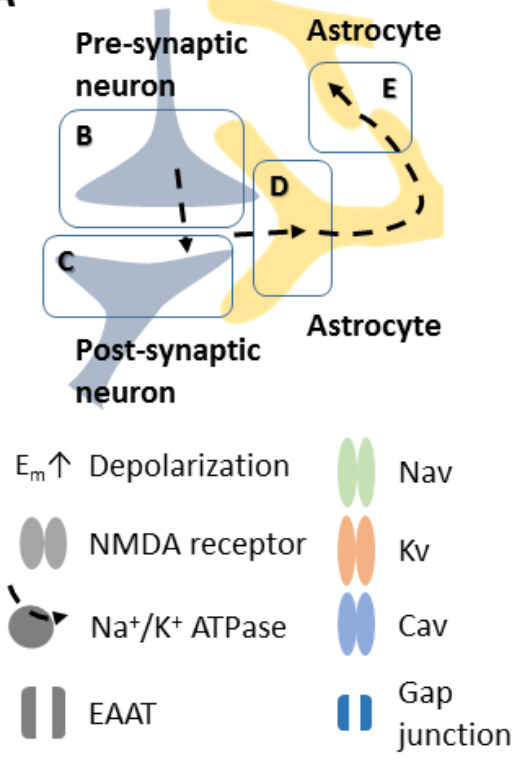

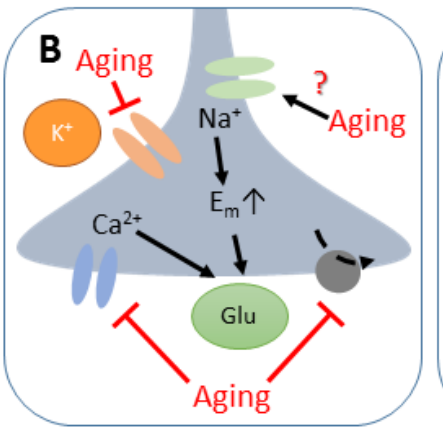
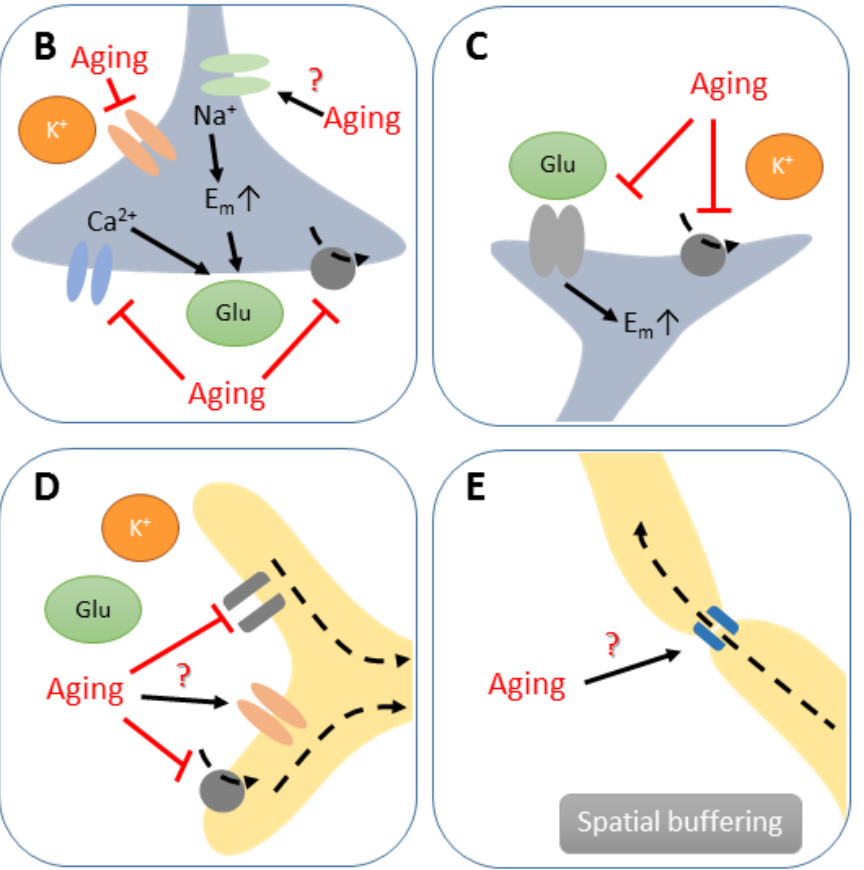

Figure 11. Conceivable targets of aging, implicated in the susceptibility of the nervous tissue to spreading depolarization (SD). A, Schematic illustration of the cellular elements hosting ion channels and transporters that are involved in SD evolution. B, Age-related dysfunction of the $\mathrm{Na}^{+} / \mathrm{K}^{+}$ATPase may contribute to $\mathrm{K}^{+}$accumulation in the interstitial space to trigger SD. While little is certain about the impact of aging on voltage-gated $\mathrm{Na}^{+}$channels $(\mathrm{Nav})$, the augmented production of reactive oxygen species with aging may alter voltage-gated $\mathrm{K}^{+}$channel $(\mathrm{Kv})$ function to reduce the excitability of the nervous tissue. Finally, aging may suppress the expression of $\mathrm{P} / \mathrm{Q}$ type voltage-gated $\mathrm{Ca}^{2+}$ channels (Cav) that may increase SD threshold. C, The age-related downregulation of NMDA receptor subunits implicated in SD may impair NMDA-based Glu signaling and SD propagation. Potassium reuptake may be also hampered by the activity of the $\mathrm{Na}^{+} / \mathrm{K}^{+}$ATPase decreased in the aging. D, Clearance mechanisms linked to astrocytes, such as $\mathrm{Na}^{+} / \mathrm{K}^{+}$ATPase activity, the expression of excitatory amino acid transporters (EAAT) and $\mathrm{K}^{+}$syphoning through Kir4.1 channels may become ineffective or lower in the aging brain, leaving higher concentration of $\mathrm{K}^{+}$and glutamate in the extracellular space, and thereby delaying repolarization. E, The spatial buffering capacity of astrocytes complementing clearance mechanisms may also be altered by age, although direct evidence in support of the suggestion is still to be acquired.

\subsection{The impact of aging on SD evolution and propagation}

As our data have revealed, the shortening of the SD-related ECoG depression appears to be evident first in the low frequency components (delta and theta bands) at the end of young adulthood (30 weeks) (Fig. 8), and concerns all frequency bands at old age (18 months old) (Makra et al., 2018). These data may depict a narrower SD wave front in space, standing for a smaller volume of nervous tissue involved in SD at a given point in time. This notion is supported by our neuroimaging studies revealing that the wave front of SD in the old cerebral cortex appears to be narrower than in the young (Farkas et al., 2011).

These electrophysiological property changes may be accomplished by the age-related modification of $\mathrm{Kv}$ and BK channel function (Sesti, 2016). P/Q type Cav channels implicated in SD evolution were shown to be affected by aging, as well. Protein levels of P/Q type Cav 
channels in synaptosomes extracted from the cerebral cortex significantly decreased at preserved synaptic density in old rats compared to adults (Iwamoto et al., 2004). Since the pharmacological inhibition of $\mathrm{P} / \mathrm{Q}$ type Cav channels was shown to suppress SD occurrence (Hoffmann et al., 2010; F. Richter et al., 2002), the decreased expression of the channel protein at old age may also impede SD evolution (Fig. 11B).

As mentioned above, the ionic movements underlying SD are also accompanied by the release of glutamate, which sustains SD by binding to and activating NMDA receptors. The decay of NMDA receptor-based signaling with aging has been repeatedly demonstrated in the context of suboptimal synaptic neurotransmission and failing cognitive performance (Kumar, 2015). It is plausible that NMDA receptor-based signaling is compromised due to oxidative-stress mounting to levels relevant for functional deterioration in the aged brain. Declining NMDA receptor function was, for example, linked to the oxidation of $\mathrm{Ca}^{2+} /$ calmodulin-dependent protein kinase II (Bodhinathan et al., 2010). Among a number of potential mechanisms that may affect NMDA receptors in aging, the expression of distinct NMDA receptor subunits was found to be subject to age-related changes, as well (Kumar, 2015). Importantly, mRNA and protein expression of the modulatory NR2A and the NR2B subunits, both implicated in SD evolution (Shatillo et al., 2015; M. Wang et al., 2012), appeared to be downregulated in the aged brain (Kumar, 2015; Magnusson et al., 2010; Zhao et al., 2009). In summary, the agerelated dysfunction of NMDA receptors may restrict SD evolution (Fig. 11C).

\subsection{The impact of aging on the recovery from SD}

As discussed above, the proper function of neuronal and astrocytic $\mathrm{Na}^{+} / \mathrm{K}^{+}$ATPase, and $\mathrm{K}^{+}$and glutamate clearance mechanisms of astrocytes significantly contribute to the cessation of SD (Leis et al., 2005; Major et al., 2017), which is hampered in the old ischemic brain with respect to the young (Clark et al., 2014; Menyhart et al., 2015).

Most studies conducted in this regard agree that the activity of the $\mathrm{Na}^{+} / \mathrm{K}^{+}$-ATPase decreases in the aging brain (Benzi et al., 1994; Chakraborty et al., 2003; Cohadon \& Desbordes, 1986; de Lores Arnaiz \& Ordieres, 2014; Kocak et al., 2002). Crude microsomal preparations must have provided evidence for the combined weakening of neuronal and astrocytic $\mathrm{Na}^{+} / \mathrm{K}^{+}$ATPase activity, because this approach is not expected to discriminate between cell types (Kocak et al., 2002). On the other hand, investigations using synaptosomes demonstrated the age-related decline in neuronal $\mathrm{Na}^{+} / \mathrm{K}^{+}$-ATPase activity, selectively (Benzi et al., 1994; Chakraborty et al., 2003). Moreover, enzyme activity decreasing with age has been linked to 
oxidative stress, which is enhanced in the aged brain (Chakraborty et al., 2003). It is noteworthy that $\mathrm{Na}^{+} / \mathrm{K}^{+}$-ATPase hyperactivity in response to ischemia is less obvious in the aging than in the adult brain (Villa et al., 2002), in agreement with the slower recovery from SD reported in old rats suffering from cerebral ischemia (Clark et al., 2014; Menyhart et al., 2015).

Apparently, astrocytes in the aged brain display morphological alterations as well as functional adaptation (Verkhratsky et al., 2016), and show characteristics of senescence-associated secretory phenotype (Salminen et al., 2011). Intuitively, age-related loss of function should include the reduction of the efficacy of astrocyte transport mechanisms, but there is only few and indirect or conflicting evidence to be lined up. As such, both transcript and protein levels of the Kir4.1 channel and the EAAT2 glutamate transporter were shown to be gradually downregulated in the pericontusional cortical region over 3 days after experimental TBI in mice, which proved to be more pronounced in the old group of animals with respect to adults (Gupta \& Kanungo, 2013). Yet, in the uninjured cortex of the same mice, aging itself increased Kir4.1 and aquaporin-4 transcript and protein levels, interpreted as an adaptive response to maintain $\mathrm{K}^{+}$and water homeostasis (Gupta \& Kanungo, 2013). Further, aquaporin-4 channels participating in astrocytic $\mathrm{K}^{+}$uptake appeared to be less polarized (i.e. more dispersed) in astrocyte end feet in old mice (Kress et al., 2014). The expression of glial fibrillary acidic protein (GFAP) increases with age (Salminen et al., 2011), and the expression of EAAT2 has been found to decrease with increasing GFAP content, at least in brain samples of Alzheimer's disease patients (Simpson et al., 2010). It is also intriguing that the amplitude of $\mathrm{Ca}^{2+}$ signaling in astrocytes in response to the activation of their NMDA receptors was found markedly decreased at old age in protoplasmic cells isolated from the rodent cortex (Lalo et al., 2011), which may indicate that aging astrocytes are less responsive to glutamate. Altogether, these data are suggestive that transport and signaling by astrocytes are altered in the old brain (Fig. $11 \mathrm{D}$ and $\mathrm{E})$. Yet it remains to be investigated to what degree astrocyte aging contributes to the recovery from $\mathrm{SD}$, delayed in the old cerebral gray matter, especially under ischemic conditions.

Finally, astrocyte network function that relies on gap junctional contacts between adjacent cells may be subject to aging. Even though the level of the most typical astrocytic gap junction protein connexin-43 seems to be maintained into old age in the rodent brain and retina, the number, size and connexin composition of astrocytic gap junction plaques may change (Cotrina et al., 2001; Mansour et al., 2013). Whether these subtle but detectable alterations in gap 
junction structure contribute to any (mal)adaptation of astrocyte network communication with aging is yet to be examined.

\subsection{SDs occurring spontaneously in response to hypoxia/ischemia}

Even though the data presented so far consistently demonstrated that SD events are less likely to occur or evolve fully with aging (Fig. 5A), the experiments relied on provoking SD experimentally (i.e. by the application of high concentration $\mathrm{KCl}$ or electrical stimulation), rather than evaluating the likelihood of spontaneous SD evolution as it takes place in acute brain injury. The distinction between experimentally triggered and spontaneously generating SDs may be important, because their pharmacology is different, and thus the share of specific ion channels in their igniting mechanisms may not be identical (Pietrobon \& Moskowitz, 2014). In addition, the share of ion channels in supporting the generation and propagation of SD is dependent on the metabolic conditions of tissue (Dreier \& Reiffurth, 2015). This is best illustrated by the observations that NMDA receptor antagonism effectively blocks SD in wellnourished tissue (Marrannes et al., 1988), but fails to do so under severe ischemic conditions (Hertle et al., 2012).

Accordingly, contemplating the age specific pattern of spontaneous SD occurrence adds new perspectives. To begin with, the earliest age when spontaneous SD was shown to emerge due to asphyxia proved to be as early as postnatal day 4 (Hansen, 1977) - in contrast with postnatal days 12-15 determined as the youngest age in which SD cab be triggered experimentally (Bures, 1957; Frank Richter et al., 1998; Schade, 1959). The impediment for SD to evolve in such a young brain was, however, confirmed by a remarkable 20 min delay of SD onset with respect to asphyxia initiation, in contrast with the 1.5 min delay observed in adult rats (Hansen, 1977) - the longer delay possibly indicating a better tolerance of the nervous tissue against deleterious insults.

Towards the other end of the life span, the frequency of spontaneous, recurrent SDs was, likewise, found to be significantly lower in the old (23-25 months old) than in young (1.5 months old) and the middle-aged (9 months old) rat brain in a model of focal cerebral ischemia (Clark et al., 2014). This finding stands in agreement with the present and the above mentioned data, gathered using experimental SD initiation. The reason for the low SD frequency at old age could not be identified with certainty, but SDs that are often long-lasting in the old brain considerably postponed - if not altogether prevented - the occurrence of subsequent SDs, which offered a plausible explanation (Clark et al., 2014). Indeed, the duration 
of transient SD triggered under ischemia appeared to be longer-lasting in the old rat brain as compared with the young (Menyhart et al., 2015). Further, in the focal ischemia model not only the higher incidence of long-lasting SDs was characteristic among the old animals than in the younger ones, but also the larger cortical surface involved in these prolonged SD events (Clark et al., 2014). Apparently, the recovery from SD in the old brain appears to be hampered. This may point towards or underlie the increased vulnerability of the old brain to ischemic conditions, and the pathophysiologic role SD is suggested to play in injury progression.

Curiously, in another study, the generation of a spontaneous SD in immediate response to bilateral common carotid artery occlusion was more frequently encountered in old animals than in their young counterparts (Menyhart et al., 2017). Reflecting on the evidence presented here so far, this observation may seem perplexing at first, yet it is, in fact, complementary, and makes the interpretation of existing data more subtle. It must be appreciated that the spontaneous occurrence of SD in these experiments was strongly associated with a severe drop of perfusion (to 7-23\% of baseline flow), rather than with age itself, the serious perfusion deficit being more frequent among the old rats (Menyhart et al., 2017). Taken together, we postulate that irrespective of the innate, age-related electrophysiological threshold of SD elicitation, a sudden, large drop of cortical perfusion (i.e. CBF below $20 \%$ ) will inevitably bring on SDs in the old brain, as well as in the young. We also believe that even though it is more difficult to trigger SDs in the aging brain, once SD is elicited during ischemia, it is persistent in old age, and its long duration may indicate increased metabolic crisis (Farkas \& Bari, 2014), supporting the well-established high mortality and poor functional outcome of older stroke patients $(\mathrm{R}$. L. Chen et al., 2010).

\subsection{Susceptibility to SD and neurological diseases}

\subsubsection{Migraine and ischemic stroke in young adults}

The age of the animals investigated in the present study (i.e.: 7-10 vs. 30 weeks) corresponds well with human adolescence and young adulthood (Sengupta, 2013). Adolescents and young adults are at increased risk of developing migraine (Victor et al., 2010). Migraine is a multifactorial disease characterized by a unilateral, pulsating headache with moderate to severe intensity, lasting 4-72h ("Headache Classification Committee of the International Headache Society (IHS) The International Classification of Headache Disorders, 3rd edition," 2018), often compelling patients to take sick days off, and considering the relatively high prevalence (Smitherman et al., 2013), it has a substantial socioeconomic and psychosocial impact. Migraine aura is a complex neurophysiological phenomenon, characterized by mainly visual 
scotomas, sensory or sometimes speech disturbances, which usually precede the headache. Migraine in general can be divided to two types: migraine without aura (MO) and migraine with aura (MA), although MA patients may experience attacks with migraine like headache without a preceding aura phase, reflecting the complexity of the disease.

SD has been accepted as the pathophysiological phenomenon behind migraine aura, since post SD oligemia was detected by PET and fMRI in migraine patients during MA attacks (S. P. Chen \& Ayata, 2016; Hadjikhani et al., 2001; Lauritzen et al., 1983) and the velocity of SD has been found remarkably similar to that of migraine aura (Goadsby et al., 2017; Milner, 1958). It is believed that the concomitant transient depression of cerebral activity (registered via ECoG) is likely responsible for the transient neurological symptoms. In addition to triggering aura, SD could also be responsible for the evolution of the headache itself (Ayata, 2009), by activating the trigemino-vascular system, which promotes SD susceptibility a marker for migraine attack patterns in experimental studies (Ayata, 2009).

Migraine has been long considered benign and void of long-term consequences supported by experimental evidence suggesting that SDs under physiological conditions pose close to none threat to the nervous tissue (Nedergaard \& Hansen, 1988). Yet, MRI studies have confirmed, that motion processing cortical areas (e.g.: V3A) and the somatosensory cortex are significantly thicker in migraineurs (both MA and MO). These dysplastic areas might exhibit increased cortical excitably, lower local threshold of excitability and act as triggering points of SDs or, inversely, be the plastic reaction of the nervous tissue to repetitive pain (DaSilva et al., 2007). Intriguingly, the somatosensory cortical area was also recognized as the primary origin of SDs in humans following SAH and rodents exposed to focal cerebral ischemia, which was attributed to the increased susceptibility of this area due to increased neuron to glia ratio (Bogdanov et al., 2016; von Bornstadt et al., 2015).

More importantly, MA is increasingly accepted as a definitive risk factor for ischemic stroke, with meta-analyses showing more than two-fold odds-ratio increase (Schurks et al., 2009)'(Etminan et al., 2005)'(Spector et al., 2010), especially in young adults without classical stroke risk factors (Pezzini et al., 2011). Other studies involving migraine patients showed, that MA may increase stroke mortality by $40 \%$ (Gudmundsson et al., 2010) and accelerate ischemic lesion consolidation - measured by diffusion/perfusion deficit MRI (Mawet et al., 2015a). In addition to raising stroke risk, patients with MA are at increased risk to develop white matter lesions in the posterior circulatory and infratentorial region (Kruit et al., 2006; Kruit et al., 
2004). CADASIL (Cerebral Autosomal Dominant Arteriopathy with Subcortical Infarcts and Leukoencephalopathy) is an important cornerstone of the MA/ischemic stroke connection. It is a rare genetic disease caused by mutations of the NOTCH3 gene, a cell membrane receptor and characterized by a systemic, non-amyloid, non-atherosclerotic vasculopathy, with subcortical lacunar infarcts and white matter lesions ("Headache Classification Committee of the International Headache Society (IHS) The International Classification of Headache Disorders, 3rd edition," 2018). One of the earliest symptoms of CADASIL is MA, where the aura can be both classical and atypical. The evolution of MA in CADASIL remains incompletely understood, however recent experimental findings have shown that intra-arterial air microbubbles and microparticles are able to induce SDs in mice with or without generation of infarcts (Bere et al., 2014b; Nozari et al., 2010), it is thus conceivable that some migraine attacks might be triggered by microlesions of vascular origin.

Taken together, increased susceptibility to SD might explain the increased stroke risk in migraine patients (Mawet et al., 2015b)'(Guidetti et al., 2014). Young adults are usually not exposed to classical cardiovascular risk factors, and even though SD threshold may be low, an SD igniting stimulus is not present. The most common cause of cerebral ischemia in young adults are (i) patent foramen ovale (leading to paradox embolization), (ii) connective tissue abnormalities (leading to spontaneous carotid dissection) and (iii) inherited/acquired hypercoagulable state (Pezzini et al., 2011). In a subset of MA patients, one of these conditions might lead to transient regional hypoperfusions (Nozari et al., 2010) or silent microlesions (Bere et al., 2014b). In combination with increased neuronal excitability and SD susceptibility at young age, these small infarcts may provoke $\mathrm{SD}$, and thus migraine like symptoms. In contrast, the relatively higher SD threshold in the elderly is probably counteracted by increased cardiovascular risk, vascular rarefication, decrease of collateral circulation and other comorbidity leading to worse stroke outcomes (Saposnik et al., 2008). MA patients however may retain their SD susceptibility throughout their life, leading to worse stroke outcomes than the rest of the population (Gudmundsson et al., 2010), due to the role of SD in secondary injury.

\subsubsection{Subarachnoid hemorrhage}

$\mathrm{SAH}$, especially due to aneurysm rupture, represents an intriguing duality regarding epidemiology: SAH has the lowest prevalence out of all the major stroke subtypes (Feigin et al., 2003), but still poses considerable threat regarding to the potential years lost, which are comparable with that of ischemic stroke or intracerebral hemorrhage (de Rooij et al., 2013; Johnston et al., 1998). SAH is one of the most common types of stroke in young adults, and has 
a high mortality and permanent disability rate. During the days following the initial injury, focal neurological deteriorations and new ischemic lesions might arise unpredictably, termed delayed cerebral infarctions (DCI), significantly impairing clinical outcome. DCI leads to the worsening of neurological symptoms days (typically between $5-14^{\text {th }}$ day) following the hemorrhage, with corresponding new ischemic lesions. DCI has been traditionally attributed to vasospasm in the proximal large vessels, as vasospasm is present in the vast majority of SAH patients (Rowland et al., 2012) Younger age is an established risk factor for proximal large artery vasospasm (Charpentier et al., 1999; Rabb et al., 1994) and a probable risk factor for DCI (Crobeddu et al., 2012; de Rooij et al., 2013; Magge et al., 2010). Vasospasm is more likely to occur at younger age, because the loss of vessel wall elasticity, atherosclerotic changes and impaired neurovascular coupling typical of old age may collectively hamper the effective control of vascular tone, including vasoconstriction (D'Esposito et al., 2003).

Recent studies however have shown, that proximal vasospasm is but one mechanism implicated in the development of DCI. Formation of microthrombi and microvascular hypoperfusion have been also recognized to contribute to DCI (Rowland et al., 2012). Importantly, SD clusters, which might provoke inverse hemodynamic responses and concomitant tissue hypoxia, have been associated with DCI in SAH patients (Dreier et al., 2006), (Bosche et al., 2010; Dreier et al., 2009). For example, in a small, prospective cohort, high number of SDs and the total depolarized time was closely associated with DCI development, independent of vasospasm (Woitzik et al., 2012).

We have found that the inherent susceptibility of the nervous tissue to SD appears to be the lowest in young adults. Taken the evidence provided in the present section, the decreased threshold of SD in young patients may put them at a higher risk to develop DCI.

\subsubsection{Traumatic brain injury}

Traumatic brain injury is the leading cause of permanent disability and death in the pediatric and adolescent population (Thurman, 2016) and a major cause of symptomatic epilepsy in general. In animal models of TBI, SDs occur spontaneously for hours following the initial insult. It has been speculated that SDs are triggered by high extracellular $\mathrm{K}^{+}$, low $\mathrm{CBF}$ (Sunami et al., 1989) or increased intracranial pressure (Rogatsky et al., 2003), complying with the association between SD and decreased cerebral perfusion pressure (Sukhotinsky et al., 2008). At present, there is no clear evidence of the injurious potential of the early (i.e. within hours after the impact) SD events in TBI models, since histological confirmation of any SD-related 
injury following TBI in mice failed (von Baumgarten et al., 2008). However, a two-photon microscopy investigation conducted in a similar model revealed permanent dendritic beading following repetitive SDs (Sword et al., 2013).

The occurrence of SD in the injured human brain was first confirmed in a TBI patient (Mayevsky et al., 1996). Since then, TBI patients have become a group of patients most represented in SD research, because the frequent need for a craniotomy offers the opportunity to place subdural electrodes on the exposed cortical surface to monitor SDs. In these patients, SDs are often observed following TBI for at least 7 days and one study recorded recurrent SDs in 57\% of the patients examined (Hartings et al., 2011a). The origin of the first SD wave following TBI is believed to be the primary injury site (Toth et al., 2016), and the recurrence of SDs has been associated with the decrease of mean arterial pressure or the increase in core temperature (Hartings et al., 2009). Among the SDs recorded, the prolonged events predict significantly poorer long-term outcome (Hartings et al., 2011a; Hartings et al., 2011b). In addition, SDs coupled with inverse neurovascular responses were shown to coincide with the failure of cerebral autoregulation and the extension of secondary injury (Hinzman et al., 2014).

TBI has a peak incidence during adolescence due to motor-vehicle accidents (Bruns \& Hauser, 2003). Intriguingly, in one clinical study, SD incidence was found to be higher in younger patients following TBI (or ICH) (Fabricius et al., 2006). In view of these reports, our present data showing the lowest threshold of SD at adolescent or young adult age underscores the importance of SDs in young TBI patients. 


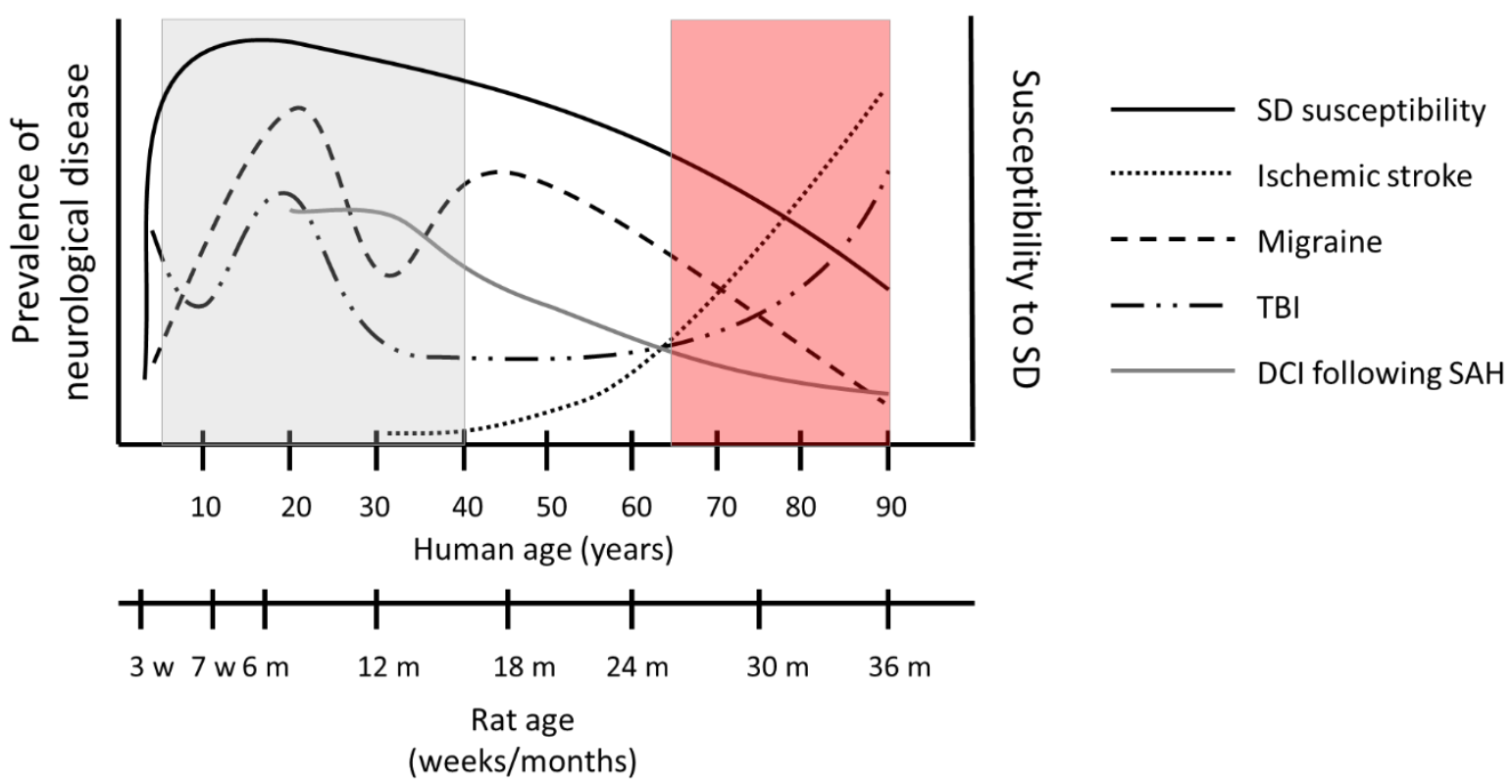

Figure 12. The age-related alteration in the prevalence of neurological diseases, in which SD is implicated as a pathophysiological phenomenon. Gray area highlights the time period, over which the prevalence of neurological diseases, such as migraine, traumatic brain injury (TBI) and delayed cerebral ischemia (DCI) following subarachnoid hemorrhage (SAH) is increased. Migraine prevalence has a secondary peak around mid-life, whereas prevalence of TBI and ischemic stroke steeply rises after 70 years of age (red area). Importantly, even though susceptibility to SD decreases with advancing age, ischemic stroke risk increases, together with prolonged SDs and worse neurological outcome. Note, that data underscoring the modification of SD susceptibility over the lifespan are predominantly based on experimental SD elicitation. In order to compare data from clinical and experimental research, the secondary x-axis represents the rat lifespan matched with human (Sengupta, 2013).

However, even though the longer cumulative duration of recurrent SDs was associated with the expansion of tissue damage in focal ischemia (Dijkhuizen et al., 1999), this does not directly infer that the longer cumulative duration is the outcome of a higher number of events - indeed, the duration of individual SD events can vary considerably (Dreier, 2011). Further, we have previously demonstrated that although only a few SDs occur spontaneously in the aged ischemic brain, these events cause more damage than a higher number of SDs in the young brain, as indicated by a remarkable delay of repolarization after SD and the associated evolution of perfusion deficit (Clark et al., 2014). Taken together, the low threshold of SD elicitation indicates the brain's high susceptibility for SD generation at young age, but it may not directly put the tissue at higher risk for SD-related injury. This notion needs to be explored further in subsequent experimental and clinical studies.

\subsection{Role of prostanoids in the cerebral blood flow response to SD}

$\mathrm{SD}$ related $\mathrm{CBF}$ response is has been the subject of much investigation (Ayata \& Lauritzen, 2015). Yet, it has remained a considerable challenge to identify the share of individual signaling cascades in the regulation of the SD related CBF response, because a great variety of vasoactive compounds are released on SD occurrence, including metabolites (e.g. adenosine, lactate), neurotransmitters (e.g. glutamate) or peptides (e.g. CGRP) (Ayata \& Lauritzen, 2015). We have 
contemplated on the role of vasodilator prostanoids in the mediation of the CBF response, because they account for at least $50 \%$ of the functional hyperemia in response to physiological somatosensory stimulation (Cauli \& Hamel, 2010). In physiological neurovascular coupling, the major pathway leading to vasodilator prostanoid synthesis involves COX-2, expressed in cortical pyramidal neurons (Lacroix et al., 2015), and located in perivascular nerve terminals along intraparenchymal penetrating arterioles and capillaries (H. Wang et al., 2005). COX-2 derived vasoactive products have emerged as mediators of functional hyperemia to somatosensory stimulation (Cauli \& Hamel, 2010; Niwa et al., 2000). In contrast with the COX2 route, the role of the constitutive COX-1 enzyme (which, in the context of physiological neurovascular coupling, is argued to be expressed in astrocytes) in shaping the CBF response to neuronal activity has remained controversial (Howarth, 2014). Selective COX-1 inhibition blocked the evolution of hyperemia in response to odorant stimulation (Petzold et al., 2008), or uncaging of $\mathrm{Ca}^{2+}$ in perivascular astrocytic endfeet (T. Takano et al., 2006), yet it exerted no impact on the CBF response to whisker stimulation (Lecrux et al., 2011; Niwa et al., 2001; Rosenegger et al., 2015).

Of the subsequent phases of the CBF response to SD (Ayata \& Lauritzen, 2015), we focused on the peak hyperemic and post-SD oligemic elements. It is important to note that our pharmacological manipulations had no significant effect on pre-SD baseline CBF levels, indicating that the results were not affected by cerebral perfusion changes. The first significant observation of the current study is that EP4 receptor antagonism reduced peak hyperemia, and augmented post-SD oligemia of the CBF response to SD in the intact brain (Fig. 10B \& C). This indicates that EP4 receptor activation contributes to vasodilation during the $\mathrm{CBF}$ response to SD. It is a novel finding, as the mediation of the hyperemic element of the CBF response by prostanoids was previously thought unlikely, and COX-derived metabolites were attributed a vasoconstrictive rather than a vasodilatory role in the CBF response to SD (Ayata \& Lauritzen, 2015; Masaaki Shibata et al., 1992). At the same time, our data are consistent with a number of previous reports on the vasodilatory action of EP4 receptor activation by $\mathrm{PGE}_{2}$. For example, antagonism of EP4 receptors inhibited the $\mathrm{PGE}_{2}$-induced vasodilation of the middle meningeal artery of rats in vivo, and of the middle cerebral artery in vitro (Davis et al., 2004; Myren et al., 2010). At the concentration used here, L161,982 previously reduced the NMDA-induced dilation of cerebral arterioles, functional hyperemia to whisker stimulation (Lacroix et al., 2015), and glutamate-evoked dilation of microvessels (Hall et al., 2014). Our data thus indicate that $\mathrm{EP} 4$ receptor activation achieves $\mathrm{CBF}$ elevation in response to $\mathrm{SD}$, as well. 
The selective inhibition of COX-1 and COX-2 enzymes exerted no significant, direct effect on any of the elements of the CBF response to SD (Fig. 10), despite the expectation that COX-2 inhibition by NS-398 would decrease the magnitude of the hyperemic element as it did functional hyperemia in response to whisker stimulation previously (Niwa et al., 2000). The reason for the absence of the COX-enzyme related vasodilation may well be that during SD, the activity of COX enzymes also leads to the marked production of vasoconstrictive prostanoids, such as prostaglandin $\mathrm{F}_{2 \alpha}$ (M. Shibata et al., 1990). Inhibition of the COX enzymes as was done in our experiments therefore likely diminishes the SD-related synthesis of both vasoconstrictive and vasodilator prostanoids, causing an undiscernible net outcome on the CBF response. The results of others and of ours together show, that functional hyperemia to neuronal activation is COX-2 dependent (i.e. selective COX-2 inhibition alone reduces the peak of hyperemia) (Lacroix et al., 2015; Lecrux et al., 2011; Niwa et al., 2000), while COX-2 inhibition has no clear impact on the CBF response to SD. These data foster the assumption that the $\mathrm{CBF}$ response to $\mathrm{SD}$ is driven by mechanisms different from physiological neurovascular coupling. Taken prostanoid-based cerebral vasoregulation, it is thus conceivable that vasoconstrictive $\mathrm{COX}$ products are released at insignificant concentration during neuronal activation, and their impact is substantial during SD(Masaaki Shibata et al., 1992), obscuring the co-existing vasodilator effect of $\mathrm{PGE}_{2}$.

In the ischemic cortex, our study revealed a considerable reduction of the distinct elements of the $\mathrm{CBF}$ response to $\mathrm{SD}$, with no detectable impact of COX enzyme inhibition or EP4 receptor blockade. Consistent with the data presented here, ischemia is known to impair physiological neurovascular coupling as evidenced by the attenuation of functional hyperemia to forepaw stimulation (Jackman et al., 2011). In addition, in the acute phase of ischemia (the target of investigation here), the abundant release of metabolic mediators of vascular tone, such as adenosine, increased ADP/ATP ratio, lactate accumulation, or the dramatically elevated concentration of extracellular potassium (Cipolla, 2009) must obscure potentially still effective, finer signals of vasoregulation. It may also well be that the prostanoid-based regulation of vascular tone becomes more obvious and could prove discernable during the subacute or chronic phases of ischemia, because COX-2 mRNA expression becomes upregulated beginning 6 hours, and endothelial EP4 receptor expression is induced 4 hours after ischemia onset(Li et al., 2008; Nogawa et al., 1997).

In summary, EP4 receptor activation contributes to vasodilation during the CBF response to SD in the intact cortex, and is undetectable under the acute phase of ischemia, possibly due to 
impaired neurovascular coupling, the dominance of metabolic mediators of vascular tone, or the upregulation of the receptors only several hours later.

\section{Conclusion}

In the present study we have provided strong evidence, that the susceptibility to SD is highest during young adulthood, and that the increase of SD threshold with age coincides with the maturation of the cerebral cortex and the narrowing of the SD wave. Further on, we have shown for the first time that, in contrast with physiological neurovascular coupling, prostanoid synthesis during SD related CBF response is not solely COX-2 dependent and that $\mathrm{PGE}_{2}$ has a central role in mediating peak hyperemia and diminishing post-SD oligemia.

Given the accepted link between SD and migraine with aura, and the increased susceptibility to SD during young adulthood, when migraine has a known prevalence peak, further emphasizes the role of SD in the evolution of migraine and might provide explanation on the increased stroke risk of these patients. Since TBI and DCI following SAH have a peak incidence during the most susceptible life-span (e.g. young adulthood), and SD has an increasingly accepted role in mediating secondary injury, young TBI or SAH patients may benefit greatly from future therapeutic options, targeting SD. Further research, especially SD monitoring in patients is needed to verify these data in humans, and to direct future potential drug developments. 
References

Aitken, P. G., Jing, J., Young, J., \& Somjen, G. G. (1991). Ion channel involvement in hypoxia-induced spreading depression in hippocampal slices. Brain Res, 541(1), 7-11.

Ayata, C. (2009). Spreading depression: from serendipity to targeted therapy in migraine prophylaxis. Cephalalgia, 29(10), 1095-1114. doi: 10.1111/j.1468-2982.2009.01982.x

Ayata, C. (2010). Cortical spreading depression triggers migraine attack: pro. Headache, 50(4), 725730. doi: 10.1111/j.1526-4610.2010.01647.x

Ayata, C., \& Lauritzen, M. (2015). Spreading Depression, Spreading Depolarizations, and the Cerebral Vasculature. Physiol Rev, 95(3), 953-993. doi: 10.1152/physrev.00027.2014

Ayata, C., Shimizu-Sasamata, M., Lo, E. H., Noebels, J. L., \& Moskowitz, M. A. (2000). Impaired neurotransmitter release and elevated threshold for cortical spreading depression in mice with mutations in the alpha1A subunit of $P / Q$ type calcium channels. Neuroscience, 95(3), 639-645.

Back, T., Ginsberg, M. D., Dietrich, W. D., \& Watson, B. D. (1996). Induction of spreading depression in the ischemic hemisphere following experimental middle cerebral artery occlusion: effect on infarct morphology. J Cereb Blood Flow Metab, 16(2), 202-213. doi: 10.1097/00004647199603000-00004

Back, T., Kohno, K., \& Hossmann, K. A. (1994). Cortical negative DC deflections following middle cerebral artery occlusion and $\mathrm{KCl}$-induced spreading depression: effect on blood flow, tissue oxygenation, and electroencephalogram. J Cereb Blood Flow Metab, 14(1), 12-19. doi: 10.1038/jcbfm.1994.3

Balestrino, M., Young, J., \& Aitken, P. (1999). Block of (Na+,K+)ATPase with ouabain induces spreading depression-like depolarization in hippocampal slices. Brain Res, 838(1-2), 37-44.

Benzi, G., Gorini, A., Arnaboldi, R., Ghigini, B., \& Villa, R. F. (1994). Age-related alterations by chronic intermittent hypoxia on cerebral synaptosomal ATPase activities. J Neural Transm Suppl, 44, 159-171.

Bere, Z., Obrenovitch, T. P., Bari, F., \& Farkas, E. (2014a). Ischemia-induced depolarizations and associated hemodynamic responses in incomplete global forebrain ischemia in rats. Neuroscience, 260, 217-226. doi: 10.1016/j.neuroscience.2013.12.032

Bere, Z., Obrenovitch, T. P., Kozak, G., Bari, F., \& Farkas, E. (2014b). Imaging reveals the focal area of spreading depolarizations and a variety of hemodynamic responses in a rat microembolic stroke model. J Cereb Blood Flow Metab, 34(10), 1695-1705. doi: 10.1038/jcbfm.2014.136

Bodhinathan, K., Kumar, A., \& Foster, T. C. (2010). Intracellular redox state alters NMDA receptor response during aging through $\mathrm{Ca} 2+$ /calmodulin-dependent protein kinase II. J Neurosci, 30(5), 1914-1924. doi: 10.1523/JNEUROSCI.5485-09.2010

Bogdanov, V. B., Middleton, N. A., Theriot, J. J., Parker, P. D., Abdullah, O. M., Ju, Y. S., . . Brennan, K. C. (2016). Susceptibility of Primary Sensory Cortex to Spreading Depolarizations. J Neurosci, 36(17), 4733-4743. doi: 10.1523/JNEUROSCI.3694-15.2016

Bosche, B., Graf, R., Ernestus, R. I., Dohmen, C., Reithmeier, T., Brinker, G., . . . Members of the Cooperative Study of Brain Injury, D. (2010). Recurrent spreading depolarizations after subarachnoid hemorrhage decreases oxygen availability in human cerebral cortex. Ann Neurol, 67(5), 607-617. doi: 10.1002/ana.21943

Bruns, J., Jr., \& Hauser, W. A. (2003). The epidemiology of traumatic brain injury: a review. Epilepsia, 44 Suppl 10, 2-10.

Bures, J. (1957). The ontogenetic development of steady potential differences in the cerebral cortex in animals. Electroencephalogr Clin Neurophysiol, 9(1), 121-130.

Busch, E., Gyngell, M. L., Eis, M., Hoehn-Berlage, M., \& Hossmann, K. A. (1996). Potassium-induced cortical spreading depressions during focal cerebral ischemia in rats: contribution to lesion growth assessed by diffusion-weighted NMR and biochemical imaging. J Cereb Blood Flow Metab, 16(6), 1090-1099. doi: 10.1097/00004647-199611000-00002 
Busija, D. W., Bari, F., Domoki, F., Horiguchi, T., \& Shimizu, K. (2008). Mechanisms involved in the cerebrovascular dilator effects of cortical spreading depression. Prog Neurobiol, 86(4), 379395. doi: 10.1016/j.pneurobio.2008.09.008

Capuani, C., Melone, M., Tottene, A., Bragina, L., Crivellaro, G., Santello, M., . . Pietrobon, D. (2016). Defective glutamate and $\mathrm{K}+$ clearance by cortical astrocytes in familial hemiplegic migraine type 2. EMBO Mol Med, 8(8), 967-986. doi: 10.15252/emmm.201505944

Carlson, A. P., Abbas, M., Alunday, R. L., Qeadan, F., \& Shuttleworth, C. W. (2018). Spreading depolarization in acute brain injury inhibited by ketamine: a prospective, randomized, multiple crossover trial. J Neurosurg, 1-7. doi: 10.3171/2017.12.JNS171665

Cauli, B., \& Hamel, E. (2010). Revisiting the role of neurons in neurovascular coupling. Front Neuroenergetics, 2, 9. doi: 10.3389/fnene. 2010.00009

Cervetto, C., Alloisio, S., Frattaroli, D., Mazzotta, M. C., Milanese, M., Gavazzo, P., . . . Marcoli, M. (2013). The P2X7 receptor as a route for non-exocytotic glutamate release: dependence on the carboxyl tail. J Neurochem, 124(6), 821-831. doi: 10.1111/jnc.12143

Chakraborty, H., Sen, P., Sur, A., Chatterjee, U., \& Chakrabarti, S. (2003). Age-related oxidative inactivation of $\mathrm{Na}+\mathrm{K}+-\mathrm{ATPase}$ in rat brain crude synaptosomes. Exp Gerontol, 38(6), 705-710. doi: 10.1016/s0531-5565(03)00066-4

Charpentier, C., Audibert, G., Guillemin, F., Civit, T., Ducrocq, X., Bracard, S., . . Laxenaire, M. C. (1999). Multivariate Analysis of Predictors of Cerebral Vasospasm Occurrence After Aneurysmal Subarachnoid Hemorrhage. Stroke, 30(7), 1402-1408. doi: 10.1161/01.str.30.7.1402

Chen, G., Gao, W., Reinert, K. C., Popa, L. S., Hendrix, C. M., Ross, M. E., \& Ebner, T. J. (2005). Involvement of kv1 potassium channels in spreading acidification and depression in the cerebellar cortex. J Neurophysiol, 94(2), 1287-1298. doi: 10.1152/jn.00224.2005

Chen, R. L., Balami, J. S., Esiri, M. M., Chen, L. K., \& Buchan, A. M. (2010). Ischemic stroke in the elderly: an overview of evidence. Nat Rev Neurol, 6(5), 256-265. doi: 10.1038/nrneurol.2010.36

Chen, S. P., \& Ayata, C. (2016). Spreading Depression in Primary and Secondary Headache Disorders. Curr Pain Headache Rep, 20(7), 44. doi: 10.1007/s11916-016-0574-8

Cholet, N., Pellerin, L., Magistretti, P. J., \& Hamel, E. (2002). Similar perisynaptic glial localization for the $\mathrm{Na}+, \mathrm{K}+-\mathrm{ATP}$ ase alpha 2 subunit and the glutamate transporters GLAST and GLT-1 in the rat somatosensory cortex. Cereb Cortex, 12(5), 515-525. doi: 10.1093/cercor/12.5.515

Cipolla, M. J. (2009). The cerebral circulation. : Morgan \& Claypool Life Sciences: San Rafael, CA.

Clark, D., Institoris, A., Kozak, G., Bere, Z., Tuor, U., Farkas, E., \& Bari, F. (2014). Impact of aging on spreading depolarizations induced by focal brain ischemia in rats. Neurobiol Aging, 35(12), 2803-2811. doi: 10.1016/j.neurobiolaging.2014.06.013

Cohadon, F., \& Desbordes, P. (1986). Brain water and aging. Gerontology, 32 Suppl 1, 46-49. doi: 10.1159/000212827

Costa, C., Tozzi, A., Rainero, I., Cupini, L. M., Calabresi, P., Ayata, C., \& Sarchielli, P. (2013). Cortical spreading depression as a target for anti-migraine agents. J Headache Pain, 14, 62. doi: 10.1186/1129-2377-14-62

Cotrina, M. L., Gao, Q., Lin, J. H., \& Nedergaard, M. (2001). Expression and function of astrocytic gap junctions in aging. Brain Res, 901(1-2), 55-61.

Crobeddu, E., Mittal, M. K., Dupont, S., Wijdicks, E. F., Lanzino, G., \& Rabinstein, A. A. (2012). Predicting the lack of development of delayed cerebral ischemia after aneurysmal subarachnoid hemorrhage. Stroke, 43(3), 697-701. doi: 10.1161/STROKEAHA.111.638403

Czeh, G., Aitken, P. G., \& Somjen, G. G. (1992). Whole-cell membrane current and membrane resistance during hypoxic spreading depression. Neuroreport, 3(2), 197-200.

D'Esposito, M., Deouell, L. Y., \& Gazzaley, A. (2003). Alterations in the BOLD fMRI signal with ageing and disease: a challenge for neuroimaging. Nat Rev Neurosci, 4(11), 863-872. doi: $10.1038 / \mathrm{nrn} 1246$

DaSilva, A. F., Granziera, C., Snyder, J., \& Hadjikhani, N. (2007). Thickening in the somatosensory cortex of patients with migraine. Neurology, 69(21), 1990-1995. doi: 10.1212/01.wnl.0000291618.32247.2d 
Davis, R. J., Murdoch, C. E., Ali, M., Purbrick, S., Ravid, R., Baxter, G. S., . . Coleman, R. A. (2004). EP4 prostanoid receptor-mediated vasodilatation of human middle cerebral arteries. $\mathrm{Br} J$ Pharmacol, 141(4), 580-585. doi: 10.1038/sj.bjp.0705645

de Lores Arnaiz, G. R., \& Ordieres, M. G. (2014). Brain Na(+), K(+)-ATPase Activity In Aging and Disease. Int J Biomed Sci, 10(2), 85-102.

de Rooij, N. K., Greving, J. P., Rinkel, G. J., \& Frijns, C. J. (2013). Early prediction of delayed cerebral ischemia after subarachnoid hemorrhage: development and validation of a practical risk chart. Stroke, 44(5), 1288-1294. doi: 10.1161/STROKEAHA.113.001125

Di Cesare Mannelli, L., Marcoli, M., Micheli, L., Zanardelli, M., Maura, G., Ghelardini, C., \& Cervetto, C. (2015). Oxaliplatin evokes P2X7-dependent glutamate release in the cerebral cortex: A pain mechanism mediated by Pannexin 1. Neuropharmacology, 97, 133-141. doi: 10.1016/j.neuropharm.2015.05.037

Dichgans, M., Freilinger, T., Eckstein, G., Babini, E., Lorenz-Depiereux, B., Biskup, S., . . Strom, T. M. (2005). Mutation in the neuronal voltage-gated sodium channel SCN1A in familial hemiplegic migraine. The Lancet, 366(9483), 371-377. doi: 10.1016/s0140-6736(05)66786-4

Dickstein, D. L., Weaver, C. M., Luebke, J. I., \& Hof, P. R. (2013). Dendritic spine changes associated with normal aging. Neuroscience, 251, 21-32. doi: 10.1016/j.neuroscience.2012.09.077

Dijkhuizen, R. M., Beekwilder, J. P., van der Worp, H. B., Berkelbach van der Sprenkel, J. W., Tulleken, K. A. F., \& Nicolay, K. (1999). Correlation between tissue depolarizations and damage in focal ischemic rat brain1Published on the World Wide Web on 12 July 1999.1. Brain Res, 840(1-2), 194-205. doi: 10.1016/s0006-8993(99)01769-2

Dreier, J. P. (2011). The role of spreading depression, spreading depolarization and spreading ischemia in neurological disease. Nat Med, 17(4), 439-447. doi: 10.1038/nm.2333

Dreier, J. P., Major, S., Manning, A., Woitzik, J., Drenckhahn, C., Steinbrink, J., . . group, C. s. (2009). Cortical spreading ischaemia is a novel process involved in ischaemic damage in patients with aneurysmal subarachnoid haemorrhage. Brain, 132(Pt 7), 1866-1881. doi: 10.1093/brain/awp102

Dreier, J. P., \& Reiffurth, C. (2015). The stroke-migraine depolarization continuum. Neuron, 86(4), 902922. doi: 10.1016/j.neuron.2015.04.004

Dreier, J. P., Woitzik, J., Fabricius, M., Bhatia, R., Major, S., Drenckhahn, C., . . Strong, A. J. (2006). Delayed ischaemic neurological deficits after subarachnoid haemorrhage are associated with clusters of spreading depolarizations. Brain, 129(Pt 12), 3224-3237. doi: 10.1093/brain/awl297

Etminan, M., Takkouche, B., Isorna, F. C., \& Samii, A. (2005). Risk of ischaemic stroke in people with migraine: systematic review and meta-analysis of observational studies. BMJ, 330(7482), 63. doi: 10.1136/bmj.38302.504063.8F

Fabricius, M., Fuhr, S., Bhatia, R., Boutelle, M., Hashemi, P., Strong, A. J., \& Lauritzen, M. (2006). Cortical spreading depression and peri-infarct depolarization in acutely injured human cerebral cortex. Brain, 129(Pt 3), 778-790. doi: 10.1093/brain/awh716

Farkas, E., \& Bari, F. (2014). Spreading depolarization in the ischemic brain: does aging have an impact? J Gerontol A Biol Sci Med Sci, 69(11), 1363-1370. doi: 10.1093/gerona/glu066

Farkas, E., Obrenovitch, T. P., Institoris, A., \& Bari, F. (2011). Effects of early aging and cerebral hypoperfusion on spreading depression in rats. Neurobiol Aging, 32(9), 1707-1715. doi: 10.1016/j.neurobiolaging.2009.10.002

Feigin, V. L., Lawes, C. M. M., Bennett, D. A., \& Anderson, C. S. (2003). Stroke epidemiology: a review of population-based studies of incidence, prevalence, and case-fatality in the late 20th century. The Lancet Neurology, 2(1), 43-53. doi: 10.1016/s1474-4422(03)00266-7

Feuerstein, D., Backes, H., Gramer, M., Takagaki, M., Gabel, P., Kumagai, T., \& Graf, R. (2016). Regulation of cerebral metabolism during cortical spreading depression. J Cereb Blood Flow Metab, 36(11), 1965-1977. doi: 10.1177/0271678X15612779

Filosa, J. A., Bonev, A. D., Straub, S. V., Meredith, A. L., Wilkerson, M. K., Aldrich, R. W., \& Nelson, M. T. (2006). Local potassium signaling couples neuronal activity to vasodilation in the brain. Nat Neurosci, 9(11), 1397-1403. doi: 10.1038/nn1779 
Fordsmann, J. C., Ko, R. W., Choi, H. B., Thomsen, K., Witgen, B. M., Mathiesen, C., . . Lauritzen, M. (2013). Increased 20-HETE synthesis explains reduced cerebral blood flow but not impaired neurovascular coupling after cortical spreading depression in rat cerebral cortex. J Neurosci, 33(6), 2562-2570. doi: 10.1523/JNEUROSCI.2308-12.2013

Gagolewicz, P. J. A., R.D. . (2017). The Elusive Channel Driving Ischemic Spreading Depolarization. . Program No. 122.01. Neuroscience Meeting Planner. Washington, DC: Society for Neuroscience, 2017. Online.

Glaser, E. M., \& Van der Loos, H. (1981). Analysis of thick brain sections by obverse-reverse computer microscopy: application of a new, high clarity Golgi-Nissl stain. J Neurosci Methods, 4(2), 117125. doi: 10.1016/0165-0270(81)90045-5

Goadsby, P. J., Holland, P. R., Martins-Oliveira, M., Hoffmann, J., Schankin, C., \& Akerman, S. (2017). Pathophysiology of Migraine: A Disorder of Sensory Processing. Physiol Rev, 97(2), 553-622. doi: 10.1152/physrev.00034.2015

Grafstein, B. (1956). Mechanism of spreading cortical depression. J Neurophysiol, 19(2), 154-171. doi: 10.1152/jn.1956.19.2.154

Gudmundsson, L. S., Scher, A. I., Aspelund, T., Eliasson, J. H., Johannsson, M., Thorgeirsson, G., . . . Gudnason, V. (2010). Migraine with aura and risk of cardiovascular and all cause mortality in men and women: prospective cohort study. BMJ, 341, c3966. doi: 10.1136/bmj.c3966

Guedes, R. C., Amorim, L. F., \& Teodosio, N. R. (1996). Effect of aging on cortical spreading depression. Braz J Med Biol Res, 29(10), 1407-1412.

Guidetti, D., Rota, E., Morelli, N., \& Immovilli, P. (2014). Migraine and stroke: "vascular" comorbidity. Front Neurol, 5, 193. doi: 10.3389/fneur.2014.00193

Gupta, R. K., \& Kanungo, M. (2013). Glial molecular alterations with mouse brain development and aging: up-regulation of the Kir4.1 and aquaporin-4. Age (Dordr), 35(1), 59-67. doi: 10.1007/s11357-011-9330-5

Hadjikhani, N., Sanchez Del Rio, M., Wu, O., Schwartz, D., Bakker, D., Fischl, B., . . Moskowitz, M. A. (2001). Mechanisms of migraine aura revealed by functional MRI in human visual cortex. Proc Natl Acad Sci U S A, 98(8), 4687-4692. doi: 10.1073/pnas.071582498

Hajek, I., Subbarao, K. V., \& Hertz, L. (1996). Acute and chronic effects of potassium and noradrenaline on $\mathrm{Na}+, \mathrm{K}+-$ ATPase activity in cultured mouse neurons and astrocytes. Neurochem Int, 28(3), 335-342.

Hall, C. N., Reynell, C., Gesslein, B., Hamilton, N. B., Mishra, A., Sutherland, B. A., .. A Attwell, D. (2014). Capillary pericytes regulate cerebral blood flow in health and disease. Nature, 508(7494), 5560. doi: $10.1038 /$ nature13165

Hamming, A. M., Wermer, M. J., Umesh Rudrapatna, S., Lanier, C., van Os, H. J., van den Bergh, W. M., ... Dijkhuizen, R. M. (2016). Spreading depolarizations increase delayed brain injury in a rat model of subarachnoid hemorrhage. J Cereb Blood Flow Metab, 36(7), 1224-1231. doi: $10.1177 / 0271678 \times 15619189$

Hansen, A. J. (1977). Extracellular potassium concentration in juvenile and adult rat brain cortex during anoxia. Acta Physiol Scand, 99(4), 412-420. doi: 10.1111/j.1748-1716.1977.tb10394.x

Hansen, A. J. (1978). The extracellular potassium concentration in brain cortex following ischemia in hypo- and hyperglycemic rats. Acta Physiol Scand, 102(3), 324-329. doi: 10.1111/j.17481716.1978.tb06079.x

Hansen, A. J., \& Olsen, C. E. (1980). Brain extracellular space during spreading depression and ischemia. Acta Physiol Scand, 108(4), 355-365. doi: 10.1111/j.1748-1716.1980.tb06544.x

Hansen, A. J., \& Zeuthen, T. (1981). Extracellular ion concentrations during spreading depression and ischemia in the rat brain cortex. Acta Physiol Scand, 113(4), 437-445. doi: 10.1111/j.17481716.1981.tb06920.x

Harada, K., Kamiya, T., \& Tsuboi, T. (2015). Gliotransmitter Release from Astrocytes: Functional, Developmental, and Pathological Implications in the Brain. Front Neurosci, 9, 499. doi: 10.3389/fnins.2015.00499 
Hardingham, G. E., \& Bading, H. (2010). Synaptic versus extrasynaptic NMDA receptor signalling: implications for neurodegenerative disorders. Nat Rev Neurosci, 11(10), 682-696. doi: $10.1038 / \mathrm{nrn} 2911$

Harreveld, A. V. (1959). Compounds in Brain Extracts Causing Spreading Depression of Cerebral Cortical Activity and Contraction of Crustacean Muscle. J Neurochem, 3(4), 300-315. doi: 10.1111/j.1471-4159.1959.tb12636.x

Harris, K. M. J., F.E.; Tsao, B. . (1992). Three-dimensional structure of dendritic spines and synapses in rat hippocampus (CA1) at postnatal day 15 and adult ages: implications for the maturation of synaptic physiology and long-term potentiation. Journal of Neuroscience, 12(7), 20. doi: 10.1523/JNEUROSCI.12-07-02685.1992

Hartings, J. A., Bullock, M. R., Okonkwo, D. O., Murray, L. S., Murray, G. D., Fabricius, M., . . CoOperative Study on Brain Injury, D. (2011a). Spreading depolarisations and outcome after traumatic brain injury: a prospective observational study. Lancet Neurol, 10(12), 1058-1064. doi: 10.1016/S1474-4422(11)70243-5

Hartings, J. A., Shuttleworth, C. W., Kirov, S. A., Ayata, C., Hinzman, J. M., Foreman, B., . . Dreier, J. P. (2017). The continuum of spreading depolarizations in acute cortical lesion development: Examining Leao's legacy. J Cereb Blood Flow Metab, 37(5), 1571-1594. doi: 10.1177/0271678X16654495

Hartings, J. A., Strong, A. J., Fabricius, M., Manning, A., Bhatia, R., Dreier, J. P., . . Co-Operative Study of Brain Injury, D. (2009). Spreading depolarizations and late secondary insults after traumatic brain injury. J Neurotrauma, 26(11), 1857-1866. doi: 10.1089/neu.2009-0961

10.1089/neu.2009.0961

Hartings, J. A., Watanabe, T., Bullock, M. R., Okonkwo, D. O., Fabricius, M., Woitzik, J., . . Co-Operative Study on Brain Injury, D. (2011b). Spreading depolarizations have prolonged direct current shifts and are associated with poor outcome in brain trauma. Brain, 134(Pt 5), 1529-1540. doi: 10.1093/brain/awr048

Headache Classification Committee of the International Headache Society (IHS) The International Classification of Headache Disorders, 3rd edition. (2018). Cephalalgia, 38(1), 1-211. doi: 10.1177/0333102417738202

Helbok, R., Schiefecker, A. J., Friberg, C., Beer, R., Kofler, M., Rhomberg, P., . . Fabricius, M. (2017). Spreading depolarizations in patients with spontaneous intracerebral hemorrhage: Association with perihematomal edema progression. J Cereb Blood Flow Metab, 37(5), 18711882. doi: $10.1177 / 0271678 \times 16651269$

Herreras, O. S., G.G. (1993). Propagation of spreading depression among dendrites and somata of the same cell population. Brain Res., 610(2), 6. doi: 10.1016/0006-8993(93)91411-K

Hertle, D. N., Dreier, J. P., Woitzik, J., Hartings, J. A., Bullock, R., Okonkwo, D. O., . . Cooperative Study of Brain Injury, D. (2012). Effect of analgesics and sedatives on the occurrence of spreading depolarizations accompanying acute brain injury. Brain, 135(Pt 8), 2390-2398. doi: 10.1093/brain/aws152

Hille, B. (1992). G protein-coupled mechanisms and nervous signaling. Neuron, 9(2), 187-195. doi: 10.1016/0896-6273(92)90158-a

Hinzman, J. M., Andaluz, N., Shutter, L. A., Okonkwo, D. O., Pahl, C., Strong, A. J., . . Hartings, J. A. (2014). Inverse neurovascular coupling to cortical spreading depolarizations in severe brain trauma. Brain, 137(Pt 11), 2960-2972. doi: 10.1093/brain/awu241

Hinzman, J. M., DiNapoli, V. A., Mahoney, E. J., Gerhardt, G. A., \& Hartings, J. A. (2015). Spreading depolarizations mediate excitotoxicity in the development of acute cortical lesions. Exp Neurol, 267, 243-253. doi: 10.1016/j.expneurol.2015.03.014

Hoffmann, U., \& Ayata, C. (2013). Neurovascular coupling during spreading depolarizations. Acta Neurochir Suppl, 115, 161-165. doi: 10.1007/978-3-7091-1192-5_31 
Hoffmann, U., Dilekoz, E., Kudo, C., \& Ayata, C. (2010). Gabapentin suppresses cortical spreading depression susceptibility. J Cereb Blood Flow Metab, 30(9), 1588-1592. doi: 10.1038/jcbfm.2010.92

Hoffmann, U., Sukhotinsky, I., Eikermann-Haerter, K., \& Ayata, C. (2013). Glucose modulation of spreading depression susceptibility. J Cereb Blood Flow Metab, 33(2), 191-195. doi: $10.1038 / \mathrm{jcbfm} .2012 .132$

Howarth, C. (2014). The contribution of astrocytes to the regulation of cerebral blood flow. Front Neurosci, 8, 103. doi: 10.3389/fnins.2014.00103

Iwamoto, M., Hagishita, T., Shoji-Kasai, Y., Ando, S., \& Tanaka, Y. (2004). Age-related changes in the levels of voltage-dependent calcium channels and other synaptic proteins in rat brain cortices. Neurosci Lett, 366(3), 277-281. doi: 10.1016/j.neulet.2004.05.048

Jackman, K. A., Brait, V. H., Wang, Y., Maghzal, G. J., Ball, H. J., McKenzie, G., . . Sobey, C. G. (2011). Vascular expression, activity and function of indoleamine 2,3-dioxygenase-1 following cerebral ischaemia-reperfusion in mice. Naunyn Schmiedebergs Arch Pharmacol, 383(5), 471-481. doi: 10.1007/s00210-011-0611-4

Johnston, S. C., Selvin, S., \& Gress, D. R. (1998). The burden, trends, and demographics of mortality from subarachnoid hemorrhage. Neurology, 50(5), 1413-1418.

Kao, Y. C., Li, W., Lai, H. Y., Oyarzabal, E. A., Lin, W., \& Shih, Y. Y. (2014). Dynamic perfusion and diffusion MRI of cortical spreading depolarization in photothrombotic ischemia. Neurobiol Dis, 71, 131139. doi: 10.1016/j.nbd.2014.07.005

Karatas, H., Erdener, S. E., Gursoy-Ozdemir, Y., Lule, S., Eren-Kocak, E., Sen, Z. D., \& Dalkara, T. (2013). Spreading depression triggers headache by activating neuronal Panx1 channels. Science, 339(6123), 1092-1095. doi: 10.1126/science.1231897

Kilic, K., Karatas, H., Donmez-Demir, B., Eren-Kocak, E., Gursoy-Ozdemir, Y., Can, A., . . . Dalkara, T. (2018). Inadequate brain glycogen or sleep increases spreading depression susceptibility. Ann Neurol, 83(1), 61-73. doi: 10.1002/ana.25122

Kocak, H., Oner, P., \& Oztas, B. (2002). Comparison of the activities of $\mathrm{Na}(+), \mathrm{K}(+)$-ATPase in brains of rats at different ages. Gerontology, 48(5), 279-281. doi: 10.1159/000065249

Kress, B. T., Iliff, J. J., Xia, M., Wang, M., Wei, H. S., Zeppenfeld, D., . . . Nedergaard, M. (2014). Impairment of paravascular clearance pathways in the aging brain. Ann Neurol, 76(6), 845-861. doi: 10.1002/ana.24271

Kruit, M. C., Launer, L. J., Ferrari, M. D., \& van Buchem, M. A. (2006). Brain stem and cerebellar hyperintense lesions in migraine. Stroke, 37(4), 1109-1112. doi: 10.1161/01.STR.0000206446.26702.e9

Kruit, M. C., van Buchem, M. A., Hofman, P. A., Bakkers, J. T., Terwindt, G. M., Ferrari, M. D., \& Launer, L. J. (2004). Migraine as a risk factor for subclinical brain lesions. JAMA, 291(4), 427-434. doi: 10.1001/jama.291.4.427

Kumar, A. (2015). NMDA Receptor Function During Senescence: Implication on Cognitive Performance. Front Neurosci, 9, 473. doi: 10.3389/fnins.2015.00473

Lacroix, A., Toussay, X., Anenberg, E., Lecrux, C., Ferreiros, N., Karagiannis, A., . . Cauli, B. (2015). COX2-Derived Prostaglandin E2 Produced by Pyramidal Neurons Contributes to Neurovascular Coupling in the Rodent Cerebral Cortex. J Neurosci, 35(34), 11791-11810. doi: 10.1523/JNEUROSCI.0651-15.2015

Lalo, U., Palygin, O., North, R. A., Verkhratsky, A., \& Pankratov, Y. (2011). Age-dependent remodelling of ionotropic signalling in cortical astroglia. Aging Cell, 10(3), 392-402. doi: 10.1111/j.14749726.2011.00682.x

Lauritzen, M., Hansen, A. J., Kronborg, D., \& Wieloch, T. (1990). Cortical spreading depression is associated with arachidonic acid accumulation and preservation of energy charge. J Cereb Blood Flow Metab, 10(1), 115-122. doi: 10.1038/jcbfm.1990.14

Lauritzen, M., Skyhoj Olsen, T., Lassen, N. A., \& Paulson, O. B. (1983). Changes in regional cerebral blood flow during the course of classic migraine attacks. Ann Neurol, 13(6), 633-641. doi: 10.1002/ana.410130609 
Leão, A. A. P. (1944). Spreading Depression of Activity in the Cerebral Cortex. J Neurophysiol, 7(6), 359390. doi: 10.1152/jn.1944.7.6.359

Lecrux, C., Toussay, X., Kocharyan, A., Fernandes, P., Neupane, S., Levesque, M., . . Hamel, E. (2011). Pyramidal neurons are "neurogenic hubs" in the neurovascular coupling response to whisker stimulation. J Neurosci, 31(27), 9836-9847. doi: 10.1523/JNEUROSCI.4943-10.2011

Leis, J. A., Bekar, L. K., \& Walz, W. (2005). Potassium homeostasis in the ischemic brain. Glia, 50(4), 407-416. doi: 10.1002/glia.20145

Leo, L., Gherardini, L., Barone, V., De Fusco, M., Pietrobon, D., Pizzorusso, T., \& Casari, G. (2011). Increased susceptibility to cortical spreading depression in the mouse model of familial hemiplegic migraine type 2. PLoS Genet, 7(6), e1002129. doi: 10.1371/journal.pgen.1002129

Li, J., Liang, X., Wang, Q., Breyer, R. M., McCullough, L., \& Andreasson, K. (2008). Misoprostol, an antiulcer agent and PGE2 receptor agonist, protects against cerebral ischemia. Neurosci Lett, 438(2), 210-215. doi: 10.1016/j.neulet.2008.04.054

Liu, F., \& McCullough, L. D. (2012). Interactions between age, sex, and hormones in experimental ischemic stroke. Neurochem Int, 61(8), 1255-1265. doi: 10.1016/j.neuint.2012.10.003

Magge, S. N., Chen, H. I., Ramakrishna, R., Cen, L., Chen, Z., Elliott, J. P., . . . Le Roux, P. D. (2010). Association of a younger age with an increased risk of angiographic and symptomatic vasospasms following subarachnoid hemorrhage. J Neurosurg, 112(6), 1208-1215. doi: 10.3171/2009.9.JNS081670

Magnusson, K. R., Brim, B. L., \& Das, S. R. (2010). Selective Vulnerabilities of N-methyl-D-aspartate (NMDA) Receptors During Brain Aging. Front Aging Neurosci, 2, 11 . doi: 10.3389/fnagi.2010.00011

Major, S., Petzold, G. C., Reiffurth, C., Windmuller, O., Foddis, M., Lindauer, U., . . Dreier, J. P. (2017). A role of the sodium pump in spreading ischemia in rats. J Cereb Blood Flow Metab, 37(5), 1687-1705. doi: 10.1177/0271678X16639059

Makra, P., Menyhart, A., Bari, F., \& Farkas, E. (2018). Spectral and Multifractal Signature of Cortical Spreading Depolarisation in Aged Rats. Front Physiol, 9, 1512. doi: 10.3389/fphys.2018.01512

Malarkey, E. B., \& Parpura, V. (2008). Mechanisms of glutamate release from astrocytes. Neurochem Int, 52(1-2), 142-154. doi: 10.1016/j.neuint.2007.06.005

Mansour, H., McColm, J. R., Cole, L., Weible, M., 2nd, Korlimbinis, A., \& Chan-Ling, T. (2013). Connexin 30 expression and frequency of connexin heterogeneity in astrocyte gap junction plaques increase with age in the rat retina. PLoS One, 8(3), e57038. doi: 10.1371/journal.pone.0057038

Marrannes, R., Willems, R., De Prins, E., \& Wauquier, A. (1988). Evidence for a role of the N-methyl-daspartate (NMDA) receptor in cortical spreading depression in the rat. Brain Res, 457(2), 226240. doi: 10.1016/0006-8993(88)90690-7

Maslarova, A., Alam, M., Reiffurth, C., Lapilover, E., Gorji, A., \& Dreier, J. P. (2011). Chronically epileptic human and rat neocortex display a similar resistance against spreading depolarization in vitro. Stroke, 42(10), 2917-2922. doi: 10.1161/STROKEAHA.111.621581

Matsuura, T., \& Bureš, J. (1971). The minimum volume of depolarized neural tissue required for triggering cortical spreading depression in rat. Experimental Brain Research, 12(3), 238-249. doi: 10.1007/bf00237916

Mawet, J., Eikermann-Haerter, K., Park, K. Y., Helenius, J., Daneshmand, A., Pearlman, L., . . Ayata, C. (2015a). Sensitivity to acute cerebral ischemic injury in migraineurs: A retrospective casecontrol study. Neurology, 85(22), 1945-1949. doi: 10.1212/WNL.0000000000002166

Mawet, J., Kurth, T., \& Ayata, C. (2015b). Migraine and stroke: in search of shared mechanisms. Cephalalgia, 35(2), 165-181. doi: 10.1177/0333102414550106

Mayevsky, A., Doron, A., Manor, T., Meilin, S., Zarchin, N., \& Ouaknine, G. E. (1996). Cortical spreading depression recorded from the human brain using a multiparametric monitoring system. Brain Res, 740(1-2), 268-274. doi: 10.1016/s0006-8993(96)00874-8

Menyhart, A., Farkas, A. E., Varga, D. P., Frank, R., Toth, R., Balint, A. R., . . Farkas, E. (2018). Largeconductance $\mathrm{Ca}(2+)$-activated potassium channels are potently involved in the inverse 
neurovascular response to spreading depolarization. Neurobiol Dis, 119, 41-52. doi: 10.1016/j.nbd.2018.07.026

Menyhart, A., Makra, P., Szepes, B. E., Toth, O. M., Hertelendy, P., Bari, F., \& Farkas, E. (2015). High incidence of adverse cerebral blood flow responses to spreading depolarization in the aged ischemic rat brain. Neurobiol Aging, 36(12), 3269-3277. doi: 10.1016/j.neurobiolaging.2015.08.014

Menyhart, A., Zolei-Szenasi, D., Puskas, T., Makra, P., Orsolya, M. T., Szepes, B. E., . . Farkas, E. (2017). Spreading depolarization remarkably exacerbates ischemia-induced tissue acidosis in the young and aged rat brain. Sci Rep, 7(1), 1154. doi: 10.1038/s41598-017-01284-4

Miettinen, S., Fusco, F. R., Yrjanheikki, J., Keinanen, R., Hirvonen, T., Roivainen, R., . . Koistinaho, J. (1997). Spreading depression and focal brain ischemia induce cyclooxygenase-2 in cortical neurons through N-methyl-D-aspartic acid-receptors and phospholipase A2. Proceedings of the National Academy of Sciences, 94(12), 6500-6505. doi: 10.1073/pnas.94.12.6500

Milner, P. M. (1958). Note on a possible correspondence between the scotomas of migraine and spreading depression of Leão. Electroencephalography and Clinical Neurophysiology, 10(4), 705. doi: 10.1016/0013-4694(58)90073-7

Misonou, H. (2010). Homeostatic regulation of neuronal excitability by $\mathrm{K}(+)$ channels in normal and diseased brains. Neuroscientist, 16(1), 51-64. doi: 10.1177/1073858409341085

Mun-Bryce, S., Wilkerson, A. C., Papuashvili, N., \& Okada, Y. C. (2001). Recurring episodes of spreading depression are spontaneously elicited by an intracerebral hemorrhage in the swine. Brain Res, 888(2), 248-255. doi: 10.1016/s0006-8993(00)03068-7

Myren, M., Baun, M., Ploug, K. B., Jansen-Olesen, I., Olesen, J., \& Gupta, S. (2010). Functional and molecular characterization of prostaglandin E2 dilatory receptors in the rat craniovascular system in relevance to migraine. Cephalalgia, 30(9), 1110-1122. doi: $10.1177 / 0333102409357957$

Nakamura, H., Strong, A. J., Dohmen, C., Sakowitz, O. W., Vollmar, S., Sue, M., . . Graf, R. (2010). Spreading depolarizations cycle around and enlarge focal ischaemic brain lesions. Brain, 133(Pt 7), 1994-2006. doi: 10.1093/brain/awq117

Nedergaard, M., \& Hansen, A. J. (1988). Spreading depression is not associated with neuronal injury in the normal brain. Brain Res, 449(1-2), 395-398. doi: 10.1016/0006-8993(88)91062-1

Nicholls, D., \& Attwell, D. (1990). The release and uptake of excitatory amino acids. Trends in Pharmacological Sciences, 11(11), 462-468. doi: 10.1016/0165-6147(90)90129-v

Niwa, K., Araki, E., Morham, S. G., Ross, M. E., \& ladecola, C. (2000). Cyclooxygenase-2 Contributes to Functional Hyperemia in Whisker-Barrel Cortex. The Journal of Neuroscience, 20(2), 763-770. doi: 10.1523/jneurosci.20-02-00763.2000

Niwa, K., Haensel, C., Ross, M. E., \& ladecola, C. (2001). Cyclooxygenase-1 Participates in Selected Vasodilator Responses of the Cerebral Circulation. Circ Res, 88(6), 600-608. doi: 10.1161/01.res.88.6.600

Nogawa, S., Zhang, F., Ross, M. E., \& ladecola, C. (1997). Cyclo-Oxygenase-2 Gene Expression in Neurons Contributes to Ischemic Brain Damage. The Journal of Neuroscience, 17(8), 27462755. doi: 10.1523/jneurosci.17-08-02746.1997

Nozari, A., Dilekoz, E., Sukhotinsky, I., Stein, T., Eikermann-Haerter, K., Liu, C., . . Moskowitz, M. A. (2010). Microemboli may link spreading depression, migraine aura, and patent foramen ovale. Ann Neurol, 67(2), 221-229. doi: 10.1002/ana.21871

Obrenovitch, T. P., Urenjak, J., \& Wang, M. (2002). Nitric oxide formation during cortical spreading depression is critical for rapid subsequent recovery of ionic homeostasis. J Cereb Blood Flow Metab, 22(6), 680-688. doi: 10.1097/00004647-200206000-00006

Petzold, G. C., Albeanu, D. F., Sato, T. F., \& Murthy, V. N. (2008). Coupling of neural activity to blood flow in olfactory glomeruli is mediated by astrocytic pathways. Neuron, 58(6), 897-910. doi: 10.1016/j.neuron.2008.04.029

Pezzini, A., Grassi, M., Lodigiani, C., Patella, R., Gandolfo, C., Casoni, F., . . . Italian Project on Stroke in Young Adults, I. (2011). Predictors of migraine subtypes in young adults with ischemic stroke: 
the italian project on stroke in young adults. Stroke, 42(1), 17-21. doi: 10.1161/STROKEAHA.110.592246

Phillips, J. M., \& Nicholson, C. (1979). Anion permeability in spreading depression investigated with ion-sensitive microelectrodes. Brain Res, 173(3), 567-571.

Pietrobon, D., \& Moskowitz, M. A. (2014). Chaos and commotion in the wake of cortical spreading depression and spreading depolarizations. Nat Rev Neurosci, 15(6), 379-393. doi: $10.1038 / \mathrm{nrn} 3770$

Pinczolits, A., Zdunczyk, A., Dengler, N. F., Hecht, N., Kowoll, C. M., Dohmen, C., . . Woitzik, J. (2017). Standard-sampling microdialysis and spreading depolarizations in patients with malignant hemispheric stroke. J Cereb Blood Flow Metab, 37(5), 1896-1905. doi: 10.1177/0271678X17699629

Rabb, C. H., Tang, G., Chin, L. S., \& Giannotta, S. L. (1994). A statistical analysis of factors related to symptomatic cerebral vasospasm. Acta Neurochir (Wien), 127(1-2), 27-31.

Richter, F., Ebersberger, A., \& Schaible, H. G. (2002). Blockade of voltage-gated calcium channels in rat inhibits repetitive cortical spreading depression. Neurosci Lett, 334(2), 123-126.

Richter, F., Lehmenkühler, A., Fechner, R., Manveljan, L., \& Haschke, W. (1998). Postnatal conditioning for spreading cortical depression in the rat brain. Developmental Brain Research, 106(1-2), 217-221. doi: 10.1016/s0165-3806(98)00018-2

Rimmele, T. S., \& Rosenberg, P. A. (2016). GLT-1: The elusive presynaptic glutamate transporter. Neurochem Int, 98, 19-28. doi: 10.1016/j.neuint.2016.04.010

Risher, W. C., Ard, D., Yuan, J., \& Kirov, S. A. (2010). Recurrent spontaneous spreading depolarizations facilitate acute dendritic injury in the ischemic penumbra. J Neurosci, 30(29), 9859-9868. doi: 10.1523/JNEUROSCI.1917-10.2010

Risher, W. C., Croom, D., \& Kirov, S. A. (2012). Persistent astroglial swelling accompanies rapid reversible dendritic injury during stroke-induced spreading depolarizations. Glia, 60(11), 17091720. doi: $10.1002 /$ glia.22390

Rogatsky, G. G., Sonn, J., Kamenir, Y., Zarchin, N., \& Mayevsky, A. (2003). Relationship between intracranial pressure and cortical spreading depression following fluid percussion brain injury in rats. J Neurotrauma, 20(12), 1315-1325. doi: 10.1089/089771503322686111

Rosenegger, D. G., Tran, C. H., Wamsteeker Cusulin, J. I., \& Gordon, G. R. (2015). Tonic Local Brain Blood Flow Control by Astrocytes Independent of Phasic Neurovascular Coupling. J Neurosci, 35(39), 13463-13474. doi: 10.1523/JNEUROSCI.1780-15.2015

Rowland, M. J., Hadjipavlou, G., Kelly, M., Westbrook, J., \& Pattinson, K. T. (2012). Delayed cerebral ischaemia after subarachnoid haemorrhage: looking beyond vasospasm. Br J Anaesth, 109(3), 315-329. doi: 10.1093/bja/aes264

Sakowitz, O. W., Kiening, K. L., Krajewski, K. L., Sarrafzadeh, A. S., Fabricius, M., Strong, A. J., . . Dreier, J. P. (2009). Preliminary evidence that ketamine inhibits spreading depolarizations in acute human brain injury. Stroke, 4O(8), e519-522. doi: 10.1161/STROKEAHA.109.549303

Salminen, A., Ojala, J., Kaarniranta, K., Haapasalo, A., Hiltunen, M., \& Soininen, H. (2011). Astrocytes in the aging brain express characteristics of senescence-associated secretory phenotype. Eur J Neurosci, 34(1), 3-11. doi: 10.1111/j.1460-9568.2011.07738.x

Saposnik, G., Cote, R., Phillips, S., Gubitz, G., Bayer, N., Minuk, J., . . Stroke Outcome Research Canada Working, G. (2008). Stroke outcome in those over 80: a multicenter cohort study across Canada. Stroke, 39(8), 2310-2317. doi: 10.1161/STROKEAHA.107.511402

Satoh, M., Asai, S., Katayama, Y., Kohno, T., \& Ishikawa, K. (1999). Real-time monitoring of glutamate transmitter release with anoxic depolarization during anoxic insult in rat striatum. Brain Res, 822(1-2), 142-148.

Schade, J. P. (1959). Maturational aspects of EEG and of spreading depression in rabbit. J Neurophysiol, 22(3), 245-257. doi: 10.1152/jn.1959.22.3.245

Schurks, M., Rist, P. M., Bigal, M. E., Buring, J. E., Lipton, R. B., \& Kurth, T. (2009). Migraine and cardiovascular disease: systematic review and meta-analysis. BMJ, 339, b3914. doi: 10.1136/bmj.b3914 
Sengupta, P. (2013). The Laboratory Rat: Relating Its Age With Human's. Int J Prev Med, 4(6), 624-630.

Sesti, F. (2016). Oxidation of K(+) Channels in Aging and Neurodegeneration. Aging Dis, 7(2), 130-135. doi: 10.14336/AD.2015.0901

Shatillo, A., Salo, R. A., Giniatullin, R., \& Grohn, O. H. (2015). Involvement of NMDA receptor subtypes in cortical spreading depression in rats assessed by fMRI. Neuropharmacology, 93, 164-170. doi: 10.1016/j.neuropharm.2015.01.028

Shibata, M., Leffler, C. W., \& Busija, D. W. (1990). Cerebral hemodynamics during cortical spreading depression in rabbits. Brain Res, 530(2), 267-274.

Shibata, M., Leffler, C. W., \& Busija, D. W. (1992). Pial arteriolar constriction following cortical spreading depression is mediated by prostanoids. Brain Res, 572(1-2), 190-197. doi: 10.1016/0006-8993(92)90469-p

Simpson, J. E., Ince, P. G., Lace, G., Forster, G., Shaw, P. J., Matthews, F., . . Ageing Neuropathology Study, G. (2010). Astrocyte phenotype in relation to Alzheimer-type pathology in the ageing brain. Neurobiol Aging, 31(4), 578-590. doi: 10.1016/j.neurobiolaging.2008.05.015

Smitherman, T. A., Burch, R., Sheikh, H., \& Loder, E. (2013). The prevalence, impact, and treatment of migraine and severe headaches in the United States: a review of statistics from national surveillance studies. Headache, 53(3), 427-436. doi: 10.1111/head.12074

Somjen, G. G. (1979). Extracellular potassium in the mammalian central nervous system. Annu Rev Physiol, 41, 159-177. doi: 10.1146/annurev.ph.41.030179.001111

Somjen, G. G. (2001). Mechanisms of spreading depression and hypoxic spreading depression-like depolarization. Physiol Rev, 81(3), 1065-1096. doi: 10.1152/physrev.2001.81.3.1065

Spector, J. T., Kahn, S. R., Jones, M. R., Jayakumar, M., Dalal, D., \& Nazarian, S. (2010). Migraine headache and ischemic stroke risk: an updated meta-analysis. Am J Med, 123(7), 612-624. doi: 10.1016/j.amjmed.2009.12.021

Spong, K. E., Andrew, R. D., \& Robertson, R. M. (2016). Mechanisms of spreading depolarization in vertebrate and insect central nervous systems. J Neurophysiol, 116(3), 1117-1127. doi: 10.1152/jn.00352.2016

Steffensen, A. B., Sword, J., Croom, D., Kirov, S. A., \& MacAulay, N. (2015). Chloride Cotransporters as a Molecular Mechanism underlying Spreading Depolarization-Induced Dendritic Beading. J Neurosci, 35(35), 12172-12187. doi: 10.1523/JNEUROSCI.0400-15.2015

Sukhotinsky, I., Dilekoz, E., Moskowitz, M. A., \& Ayata, C. (2008). Hypoxia and hypotension transform the blood flow response to cortical spreading depression from hyperemia into hypoperfusion in the rat. J Cereb Blood Flow Metab, 28(7), 1369-1376. doi: 10.1038/jcbfm.2008.35

Sun, X. L., \& Hu, G. (2010). ATP-sensitive potassium channels: a promising target for protecting neurovascular unit function in stroke. Clin Exp Pharmacol Physiol, 37(2), 243-252. doi: 10.1111/j.1440-1681.2009.05190.x

Sunami, K., Nakamura, T., Kubota, M., Ozawa, Y., Namba, H., Yamaura, A., \& Makino, H. (1989). Spreading depression following experimental head injury in the rat. Neurol Med Chir (Tokyo), 29(11), 975-980.

Sword, J., Masuda, T., Croom, D., \& Kirov, S. A. (2013). Evolution of neuronal and astroglial disruption in the peri-contusional cortex of mice revealed by in vivo two-photon imaging. Brain, 136(Pt 5), 1446-1461. doi: 10.1093/brain/awt026

Takano, K., Latour, L. L., Formato, J. E., Carano, R. A., Helmer, K. G., Hasegawa, Y., . . Fisher, M. (1996). The role of spreading depression in focal ischemia evaluated by diffusion mapping. Ann Neurol, 39(3), 308-318. doi: 10.1002/ana.410390307

Takano, T., Tian, G. F., Peng, W., Lou, N., Libionka, W., Han, X., \& Nedergaard, M. (2006). Astrocytemediated control of cerebral blood flow. Nat Neurosci, 9(2), 260-267. doi: 10.1038/nn1623

Takeda, Y., Zhao, L., Jacewicz, M., Pulsinelli, W. A., \& Nowak, T. S., Jr. (2011). Metabolic and perfusion responses to recurrent peri-infarct depolarization during focal ischemia in the Spontaneously Hypertensive Rat: dominant contribution of sporadic CBF decrements to infarct expansion. $J$ Cereb Blood Flow Metab, 31(9), 1863-1873. doi: 10.1038/jcbfm.2011.62 
Tang, C. M., Dichter, M., \& Morad, M. (1990). Modulation of the N-methyl-D-aspartate channel by extracellular H+. Proceedings of the National Academy of Sciences, 87(16), 6445-6449. doi: 10.1073/pnas.87.16.6445

Tang, Y. T., Mendez, J. M., Theriot, J. J., Sawant, P. M., Lopez-Valdes, H. E., Ju, Y. S., \& Brennan, K. C. (2014). Minimum conditions for the induction of cortical spreading depression in brain slices. J Neurophysiol, 112(10), 2572-2579. doi: 10.1152/jn.00205.2014

Thurman, D. J. (2016). The Epidemiology of Traumatic Brain Injury in Children and Youths: A Review of Research Since 1990. J Child Neurol, 31(1), 20-27. doi: 10.1177/0883073814544363

Tombaugh, G. C., \& Somjen, G. G. (1996). Effects of extracellular pH on voltage-gated $\mathrm{Na}+$, K+ and Ca2+ currents in isolated rat CA1 neurons. The Journal of Physiology, 493(3), 719-732. doi: 10.1113/jphysiol.1996.sp021417

Tong, C. K., \& Chesler, M. (2000). Modulation of spreading depression by changes in extracellular pH. J Neurophysiol, 84(5), 2449-2457. doi: 10.1152/jn.2000.84.5.2449

Toth, P., Szarka, N., Farkas, E., Ezer, E., Czeiter, E., Amrein, K., . . Koller, A. (2016). Traumatic brain injury-induced autoregulatory dysfunction and spreading depression-related neurovascular uncoupling: Pathomechanisms, perspectives, and therapeutic implications. Am J Physiol Heart Circ Physiol, 311(5), H1118-H1131. doi: 10.1152/ajpheart.00267.2016

Tottene, A., Conti, R., Fabbro, A., Vecchia, D., Shapovalova, M., Santello, M., . . Pietrobon, D. (2009). Enhanced excitatory transmission at cortical synapses as the basis for facilitated spreading depression in $\mathrm{Ca}(\mathrm{v}) 2.1$ knockin migraine mice. Neuron, 61(5), 762-773. doi: 10.1016/j.neuron.2009.01.027

van den Maagdenberg, A. M., Pietrobon, D., Pizzorusso, T., Kaja, S., Broos, L. A., Cesetti, T., . . Ferrari, M. D. (2004). A Cacna1a knockin migraine mouse model with increased susceptibility to cortical spreading depression. Neuron, 41(5), 701-710.

Verkhratsky, A., Zorec, R., Rodriguez, J. J., \& Parpura, V. (2016). Astroglia dynamics in ageing and Alzheimer's disease. Curr Opin Pharmacol, 26, 74-79. doi: 10.1016/j.coph.2015.09.011

Victor, T. W., Hu, X., Campbell, J. C., Buse, D. C., \& Lipton, R. B. (2010). Migraine prevalence by age and sex in the United States: a life-span study. Cephalalgia, 30(9), 1065-1072. doi: 10.1177/0333102409355601

Villa, R. F., Gorini, A., \& Hoyer, S. (2002). ATPases of synaptic plasma membranes from hippocampus after ischemia and recovery during ageing. Neurochem Res, 27(9), 861-870.

Vinogradova, L. V. (2018). Initiation of spreading depression by synaptic and network hyperactivity: Insights into trigger mechanisms of migraine aura. Cephalalgia, 38(6), 1177-1187. doi: 10.1177/0333102417724151

von Baumgarten, L., Trabold, R., Thal, S., Back, T., \& Plesnila, N. (2008). Role of cortical spreading depressions for secondary brain damage after traumatic brain injury in mice. $J$ Cereb Blood Flow Metab, 28(7), 1353-1360. doi: 10.1038/jcbfm.2008.30

von Bornstadt, D., Houben, T., Seidel, J. L., Zheng, Y., Dilekoz, E., Qin, T., . . Ayata, C. (2015). Supplydemand mismatch transients in susceptible peri-infarct hot zones explain the origins of spreading injury depolarizations. Neuron, 85(5), 1117-1131. doi: 10.1016/j.neuron.2015.02.007

Wang, H., Hitron, I. M., ladecola, C., \& Pickel, V. M. (2005). Synaptic and vascular associations of neurons containing cyclooxygenase-2 and nitric oxide synthase in rat somatosensory cortex. Cereb Cortex, 15(8), 1250-1260. doi: 10.1093/cercor/bhi008

Wang, M., Chazot, P. L., Ali, S., Duckett, S. F., \& Obrenovitch, T. P. (2012). Effects of NMDA receptor antagonists with different subtype selectivities on retinal spreading depression. $\mathrm{Br} J$ Pharmacol, 165(1), 235-244. doi: 10.1111/j.1476-5381.2011.01553.x

Woitzik, J., Dreier, J. P., Hecht, N., Fiss, I., Sandow, N., Major, S., .. group, C. s. (2012). Delayed cerebral ischemia and spreading depolarization in absence of angiographic vasospasm after subarachnoid hemorrhage. J Cereb Blood Flow Metab, 32(2), 203-212. doi: 10.1038/jcbfm.2011.169 
Woitzik, J., Hecht, N., Pinczolits, A., Sandow, N., Major, S., Winkler, M. K., . . group, C. s. (2013). Propagation of cortical spreading depolarization in the human cortex after malignant stroke. Neurology, 80(12), 1095-1102. doi: 10.1212/WNL.0b013e3182886932

Wu, Y. J., Boissard, C. G., Greco, C., Gribkoff, V. K., Harden, D. G., He, H., .. . Dworetzky, S. I. (2003). (S)$\mathrm{N}$-[1-(3-morpholin-4-ylphenyl)ethyl]- 3-phenylacrylamide: an orally bioavailable KCNQ2 opener with significant activity in a cortical spreading depression model of migraine. J Med Chem, 46(15), 3197-3200. doi: 10.1021/jm034073f

Wulff, H., \& Zhorov, B. S. (2008). K+ channel modulators for the treatment of neurological disorders and autoimmune diseases. Chem Rev, 108(5), 1744-1773. doi: 10.1021/cr078234p

Yao, X., Smith, A. J., Jin, B. J., Zador, Z., Manley, G. T., \& Verkman, A. S. (2015). Aquaporin-4 regulates the velocity and frequency of cortical spreading depression in mice. Glia, 63(10), 1860-1869. doi: 10.1002/glia.22853

Zhao, X., Rosenke, R., Kronemann, D., Brim, B., Das, S. R., Dunah, A. W., \& Magnusson, K. R. (2009). The effects of aging on $\mathrm{N}$-methyl-D-aspartate receptor subunits in the synaptic membrane and relationships to long-term spatial memory. Neuroscience, 162(4), 933-945. doi: 10.1016/j.neuroscience.2009.05.018

Zhou, N., Rungta, R. L., Malik, A., Han, H., Wu, D. C., \& MacVicar, B. A. (2013). Regenerative glutamate release by presynaptic NMDA receptors contributes to spreading depression. J Cereb Blood Flow Metab, 33(10), 1582-1594. doi: 10.1038/jcbfm.2013.113 
Publications related to the $\mathrm{PhD}$ thesis

I. Hertelendy, P., Varga, D. P., Menyhárt, Á., Bari, F., \& Farkas, E.

Susceptibility of the cerebral cortex to spreading depolarization in neurological disease states: The impact of aging.

Neurochemistry International. 2018 In press. doi:10.1016/j.neuint.2018.10.010

IF: 3.603 


\title{
Susceptibility of the cerebral cortex to spreading depolarization in neurological disease states: The impact of aging
}

\author{
Péter Hertelendy ${ }^{\mathrm{a}, \mathrm{b}}$, Dániel P. Varga ${ }^{\mathrm{a}}$, Ákos Menyhárt ${ }^{\mathrm{a}}$, Ferenc Bari ${ }^{\mathrm{a}}$, Eszter Farkas ${ }^{\mathrm{a}, *}$ \\ a Department of Medical Physics and Informatics, Faculty of Medicine and Faculty of Science and Informatics, University of Szeged, Korányis Fasor 9, H-6720, Szeged, \\ Hungary \\ ${ }^{\mathrm{b}}$ Department of Neurology Faculty of Medicine, University of Szeged, Semmelweis u. 6, H-6725, Szeged, Hungary
}

\section{A R T I C L E I N F O}

\section{Keywords:}

Aging

Brain injury

Ischemia

Migraine

Spreading depolarization

Spreading depression

\begin{abstract}
A B S T R A C T
Secondary injury following acute brain insults significantly contributes to poorer neurological outcome. The spontaneous, recurrent occurrence of spreading depolarization events (SD) has been recognized as a potent secondary injury mechanism in subarachnoid hemorrhage, malignant ischemic stroke and traumatic brain injury. In addition, SD is the underlying mechanism of the aura symptoms of migraineurs. The susceptibility of the nervous tissue to SD is subject to the metabolic status of the tissue, the ionic composition of the extracellular space, and the functional status of ion pumps, voltage-gated and other cation channels, glutamate receptors and excitatory amino acid transporters. All these mechanisms tune the excitability of the nervous tissue. Aging has also been found to alter SD susceptibility, which appears to be highest at young adulthood, and decline over the aging process. The lower susceptibility of the cerebral gray matter to SD in the old brain may be caused by the age-related impairment of mechanisms implicated in ion translocations between the intra- and extracellular compartments, glutamate signaling and surplus potassium and glutamate clearance. Even though the aging nervous tissue is thus less able to sustain SD, the consequences of SD recurrence in the old brain have proven to be graver, possibly leading to accelerated lesion maturation. Taken that recurrent SDs may pose an increased burden in the aging injured brain, the benefit of therapeutic approaches to restrict SD generation and propagation may be particularly relevant for elderly patients.
\end{abstract}

\section{Introduction}

\subsection{Occurrence of spreading depolarization (SD) in neurological diseases}

Spreading depolarization (SD) is a wave of massive depolarization of a critical mass of neurons and presumably glia cells, which - together with a concomitant depression of spontaneous brain electrical activity propagates across the cerebral gray matter at a low rate of $2-8 \mathrm{~mm} / \mathrm{min}$ (Leao, 1944; Somjen, 2001). Not long after its discovery, SD was speculated to correspond with scotomas of migraine with aura on the basis of a similar rate of propagation (Milner, 1958), although no direct proof could be gathered at the time to indisputably support the claim. Consequently, SD was considered for decades as an experimental curiosity, or a model for neurovascular coupling, the latter due to the evolution of an associated, robust cerebral blood flow (CBF) response. The CBF response to SD generally consists of an initial, brief vasoconstriction, followed by a remarkable, transient, and then a less obvious late hyperemia, which are succeeded by a long lasting oligemia. The final, oligemic element of the CBF response is typically obvious in the non-ischemic cortex in case a prior SD event was not generated within the preceding hour. The presence and weight of each, distinct phase in the $\mathrm{CBF}$ response may vary in different species, and is subject to the metabolic state of the tissue, establishing a spectrum of CBF response types ranging from the spectacular dominance of peak hyperemia to ruling vasoconstriction known as spreading ischemia (Ayata and Lauritzen, 2015).

SD in a patient - rather than in experimental model systems - was first captured during the aura phase of a migraine attack. In fact, an epiphenomenon, spreading oligemia was revealed by positron-emission tomography (PET) (Lauritzen et al., 1983). Later the full CBF response to SD (i.e. including both hyperemia and oligemia) was confirmed in migraine patients by functional magnetic resonance imaging using blood oxygen level detection (fMRI-BOLD) techniques (Hadjikhani et al., 2001). The clinical evidence were highly significant, but still

\footnotetext{
* Corresponding author.

E-mail addresses: hertelendy.peter@med.u-szeged.hu (P. Hertelendy), vardan.bio@gmail.com (D.P. Varga), menyhartakos89@gmail.com (Á. Menyhárt), bari.ferenc@med.u-szeged.hu (F. Bari), farkas.eszter.1@med.u-szeged.hu (E. Farkas).
} 


\author{
Abbreviations \\ BK large-conductance $\mathrm{Ca}^{2+}$ activated $\mathrm{K}^{+}$channel \\ Cav voltage-gated calcium channel \\ CBF cerebral blood flow \\ DC potential direct current potential \\ DCI delayed cerebral infarctions \\ EAAT excitatory amino acid transporter \\ ECoG electrocorticogram \\ eNOS endothelial nitric oxide synthase \\ GFAP glial fibrillary acidic protein
}
IL-1 $\beta$
interleukin-1 $\beta$
Kir inward rectifier potassium channel
$\mathrm{Kv} \quad$ voltage-gated potassium channel
Nav voltage-gated sodium channel
NMDA N-methyl-D-aspartate
NO nitric oxide
nNOS neuronal nitric oxide synthase
$\mathrm{SAH}$ subarachnoidal hemorrhage
SD spreading depolarization
TBI traumatic brain injury
TNF $\alpha$ tumor necrosis factor- $\alpha$

indirect, since the $\mathrm{CBF}$ variation characteristic of SD, rather than SD itself (the primary, electrophysiological event), was identified. Still, SD has been justly acknowledged to accompany the aura phase of migraine (Ayata, 2010), and the associated transient depression of neural activity has been linked to neurological symptoms such as scintillations and scotomas (Goadsby et al., 2017; Milner, 1958). Although it is still debated, SD may sensitize the trigeminovascular system, activate meningeal nociceptors, and thereby contribute to migraine headache itself, as well (Ayata, 2009; Goadsby et al., 2017).

The first, direct indication for SD to occur in the human brain was presented by multiparametric monitoring of the cerebral cortex of severe traumatic brain injury (TBI) patients. The synchronous acquisition of CBF, extracellular $\mathrm{K}^{+}$concentration $\left(\left[\mathrm{K}^{+}\right]_{\mathrm{e}}\right.$ ), direct-current (DC)potential, electrocorticogram (ECoG) and changes in NADH redox state unequivocally confirmed that SD, in association with injury, evolves in a recurrent fashion in the human brain (Mayevsky et al., 1996, 1998). Subsequently, the systematic monitoring of SD in acute brain injury patients (i.e. TBI, subarachnoid hemorrhage - SAH, malignant ischemic stroke) took off starting with a landmark study (Strong et al., 2002). The most reliable approach to detect SD has since become the use of subdural surface electrode strips left in place for up to several days after the neurosurgical intervention to alleviate the primary traumatic or ischemic insult (Dreier et al., 2017). Because the recording of SDs with scalp electrodes requires further validation (Hartings et al., 2014), SD monitoring at present remains predominantly invasive, limited to acute brain injury patients requiring craniotomy. These studies keep delivering highly valuable data on the pattern of evolution and injurious potential of SD, and promote SD as an indicator or mediator of ongoing secondary damage (Dreier et al., 2017; Hartings et al., 2017).

\subsection{Contribution of SD to lesion progression}

Following SAH, delayed cerebral infarctions (DCI) (i.e. focal neurological deterioration, new ischemic lesions and related neurological symptoms) arise unpredictably, typically 5-14 days following the initial injury. DCI was originally attributed to vasospasm in the proximal large vessels, but, more recently, the additional contribution of microthrombus formation and microvascular hypoperfusion was revealed (Rowland et al., 2012). Microvascular hypoperfusion, in particular, was suggested to be caused by clusters of SD (Dreier et al., 2006). Such SDs may be coupled with inverse hemodynamic response and concomitant tissue hypoxia, deepening the metabolic crisis of the tissue and leading to ischemic lesion progression (Bosche et al., 2010; Dreier et al., 2009). The inverse hemodynamic response to SD has also been observed in TBI patients, and presented as a novel mechanism of secondary brain injury (Hinzman et al., 2014). As further support for the injurious potential of recurrent SDs, the high number of SDs and the total depolarization time were closely associated with DCI development, independent of vasospasm (Dreier et al., 2006; Woitzik et al., 2012).

Recently, repetitive SDs - in clusters or alone - have also been identified in patients suffering malignant ischemic stroke (Pinczolits et al., 2017; Woitzik et al., 2013). In focal cerebral ischemia, repetitive
SDs are thought to arise from the border of the penumbra and the ischemic core (Bere et al., 2014; Kao et al., 2014), probably initiated by hypotensive or hypoxic transients (von Bornstadt et al., 2015). These SDs may increase the chance of infarct maturation by converting viable penumbra tissue to the core beyond repair (Hartings et al., 2017). The coincidence between the severity of ischemic damage and SD occurrence was corroborated by the linear correlation between the total depolarization time or SD frequency with infarct maturation in rodent focal ischemia models (Back et al., 1994, 1996; Dijkhuizen et al., 1999; Takano et al., 1996). In order to determine the direction of causality, SDs elicited experimentally distant to ischemic foci were shown to propagate to penumbra-like tissue and increase the size of the ischemic infarct (Busch et al., 1996), verifying that SDs may contribute to ischemic lesion progression.

\subsection{Age as a risk factor for neurological diseases in which SD is relevant}

Taken that SD emerges as a potent mechanism of secondary brain injury in patients, it is of great interest what conditions favor SD occurrence. In neurological disorders implicating SD (i.e. migraine with aura, or acute brain injury including TBI, SAH, and malignant ischemic stroke), age is known as an independent risk factor for the incidence and prevalence of the disorders (Fig. 1). For example, the age-dependent prevalence of migraine has been shown to be bimodal, as migraine incidence peaks at the age of 19 and 48 years in men, and at the age of 25 and 50 in women (Victor et al., 2010) (Fig. 1). Among the general population, TBI has a peak incidence during childhood (falls), adolescence (motor-vehicle accidents) and geriatric age (falls) (Bruns and Hauser, 2003) (Fig. 1). Further, SAH is one of the most common types of stroke in young adults, and younger age is an established risk factor

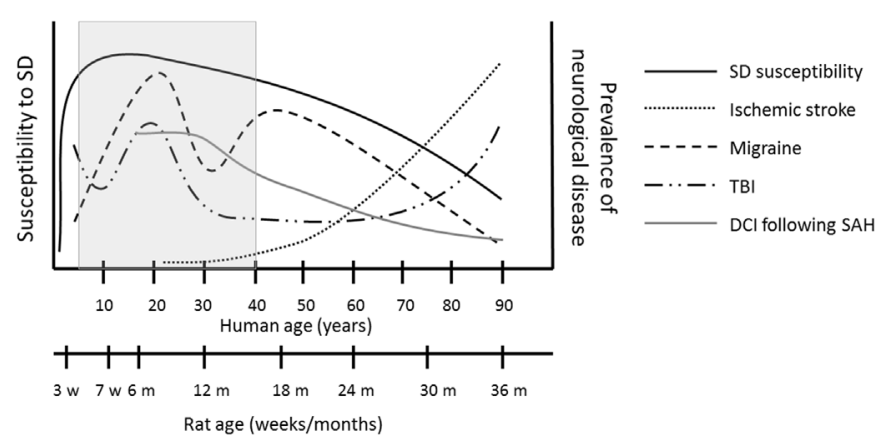

Fig. 1. The age-related alteration of spreading depolarization (SD) susceptibility against the prevalence of neurological diseases, in which SD is implicated as a pathophysiological phenomenon. Note, that data underscoring the modification of SD susceptibility over the lifespan are predominantly based on experimental SD elicitation. In order to compare data from clinical and experimental research, the secondary $\mathrm{x}$-axis represents the rat lifespan matched with human (Sengupta, 2013). Gray area highlights the time period, over which increased SD susceptibility coincides with the increased prevalence of neurological diseases, such as migraine, traumatic brain injury (TBI) and delayed cerebral ischemia (DCI) following subarachnoid hemorrhage (SAH). 
for secondary lesion progression in $\mathrm{SAH}$, caused by proximal large artery vasospasm (Charpentier et al., 1999; Rabb et al., 1994) and DCI (Crobeddu et al., 2012; de Rooij et al., 2013; Magge et al., 2010) (Fig. 1). Finally, aging significantly predicts poor patient outcomes after ischemic stroke (Chen et al., 2010; Liu and McCullough, 2012). The incidence and poor outcome of stroke steeply rises with age (Chen et al., 2010) (Fig. 1). In this context, the impact of age on stroke pathophysiology has been the target of intensive research in order to understand the reason for the increased susceptibility of the aged brain to stroke-related injury, yet the potential contribution of SD has remained largely unexplored.

In recent years, outstanding and comprehensive reviews focusing on different aspects of SD have been published: the pharmacological profile of SD (Pietrobon and Moskowitz, 2014); the linkage between migraine, stroke and SD (Dreier and Reiffurth, 2015); the aspects of hemodynamic response to SD (Ayata and Lauritzen, 2015) and the pivotal role of SD during ischemic injury (Hartings et al., 2017). Here we set out to provide an overview on the susceptibility of the nervous tissue to $\mathrm{SD}$, with a principal focus on age.

\section{Aging and the susceptibility of the nervous tissue to SD}

\subsection{SD triggered experimentally}

The neonatal brain appears to be too immature to sustain experimentally triggered SD. In the intact rat cortex, SD can be first initiated from postnatal days 12-15 (Bures, 1957; Richter et al., 1998; Schade, 1959). The threshold of SD elicitation was thought to decrease until adulthood, and expected to decrease further with aging, theoretically due to the shrinkage of the extracellular space (Somjen, 2001). However, the latter view on aging appears to be superseded by accumulating experimental evidence. First, the rate of SD propagation was shown to decelerate in the aging rodent brain (Guedes et al., 1996). Also, increasingly higher concentration of $\mathrm{KCl}$ was required to trigger SD in brain slices obtained from middle-aged rats with respect to young adults (Maslarova et al., 2011), and in the old versus young anesthetized rat cerebral cortex (Menyhart et al., 2015, 2017b). Further, the same, incessant, standard trigger $(1 \mathrm{M} \mathrm{KCl})$ produced a lower number of recurrent SDs in the middle-aged with respect to the young adult cerebral cortex in anesthetized rats (Farkas et al., 2011). Finally, a more sensitive, refined experimental approach dissected that between the ages of 7-30 weeks of rats - corresponding to adolescence and young adulthood in humans (Sengupta, 2013) - progressively increasing electrical charge was necessary to elicit SD, especially when the cortex suffered of global forebrain ischemia (Hertelendy et al., 2017). In the non-ischemic cortex, the elevating electric threshold of SD elicitation coincided with increasing dendritic spine density, which might outline an association between SD threshold and the fine histological and possibly neurochemical organization of the cortex (Hertelendy et al., 2017).

In further support for the concept that the aged nervous tissue is less able to sustain SD, additional experimental results may be lined up. In the full band ECoG, SD is seen as a transient, spreading depression of activity (Leão, 1944) - unless the ECoG is already isoelectric prior to SD occurrence due to a severe insult (Hartings et al., 2011). The duration of the ECoG depression in the non-ischemic rat cerebral cortex may last for over $2 \mathrm{~min}$ in the young, but for only half of this time duration in middle-aged animals (Farkas et al., 2011). The shortening of the SDrelated ECoG depression appears to be evident first in the low frequency components (delta and theta bands) at middle-age (7 months old) (Hertelendy et al., 2017), and concerns all frequency bands at old age (18 months old) (Makra, 2018). These data have been interpreted to depict a narrower SD wave front in space, standing for a smaller volume of nervous tissue involved in SD at a given point in time (Farkas et al., 2011; Hertelendy et al., 2017; Makra, 2018).

Another read-out to be taken as an indicator for the susceptibility of the nervous tissue to SD is the distance covered by SD before coming to a halt. Indeed, some SD events are gradually extinguished over their course of propagation, rather than traversing the entire cortical surface, as demonstrated by multimodal experimental imaging studies (Bere et al., 2014; Kaufmann et al., 2017; Menyhart et al., 2017a). Even though not documented so far, we observed that in old (18 months old) rats, experimentally triggered SDs are more likely to terminate after having propagated only a few millimeters away from the site of elicitation than in young ( 2 months old) rats.

\subsection{SD that occurs spontaneously in response to hypoxia/ischemia}

Even though these studies consistently demonstrated that SD events are less likely to occur or evolve fully with aging (Fig. 1), the experiments relied on provoking SD experimentally (i.e. by the application of high concentration $\mathrm{KCl}$ or electrical stimulation), rather than evaluating the likelihood of spontaneous SD evolution as it takes place in acute brain injury. The distinction between experimentally triggered and spontaneously generating SDs may be important, because their pharmacology is different, and thus the share of specific ion channels in their igniting mechanisms may not be identical (Pietrobon and Moskowitz, 2014). In addition, the share of ion channels in supporting the generation and propagation of SD is dependent on the metabolic conditions of tissue (Dreier and Reiffurth, 2015). This is best illustrated by the observations that NMDA receptor antagonism effectively blocks SD in well-nourished tissue (Marrannes et al., 1988), but fails to do so under severe ischemic conditions (Hertle et al., 2012). Accordingly, contemplating the age specific pattern of spontaneous SD occurrence adds new perspectives. To begin with, the earliest age when spontaneous SD was shown to emerge due to asphyxia proved to be as early as postnatal day 4 (Hansen, 1977) - in contrast with postnatal days 12-15 determined as the youngest age in which SD can be triggered experimentally (Bures, 1957; Richter et al., 1998; Schade, 1959). The impediment for SD to evolve in such a young brain was, however, confirmed by a remarkable $20 \mathrm{~min}$ delay of SD onset with respect to asphyxia initiation, in contrast with the 1.5 min delay observed in adult rats (Hansen, 1977) - the longer delay possibly indicating a better tolerance of the nervous tissue against deleterious insults. Towards the other end of the life span, the frequency of spontaneous, recurrent SDs was, likewise, found significantly lower in the old (23-25 months old) than in young ( 1.5 months old) and the middle-aged ( 9 months old) rat brain in a model of focal cerebral ischemia (Clark et al., 2014). This finding stands in agreement with the above data gathered using experimental SD initiation. The reason for the low SD frequency at old age could not be identified with certainty, but SDs that are often longlasting in the old brain considerably postponed - if not altogether prevented - the occurrence of subsequent SDs, which offered a plausible explanation (Clark et al., 2014). Indeed, the duration of transient SD triggered under ischemia appeared to be longer-lasting in the old rat brain as compared with the young (Menyhart et al., 2015). Further, in the focal ischemia model not only the higher incidence of long-lasting SDs was characteristic among the old animals than in the younger ones, but also the larger cortical surface involved in these prolonged SD events (Clark et al., 2014). Apparently, the recovery from SD in the old brain appears to be hampered. This may point towards or underlie the increased vulnerability of the old brain to ischemic conditions, and the pathophysiologic role SD is suggested to play in injury progression.

Curiously, in another study, the generation of a spontaneous SD in immediate response to bilateral common carotid artery occlusion was more frequently encountered in old animals than in their young counterparts (Menyhart et al., 2017b). Reflecting on the evidence presented here so far, this observation may seem perplexing at first, yet it is, in fact, complementary, and makes the interpretation of existing data more subtle. It must be appreciated that the spontaneous occurrence of SD in these experiments was strongly associated with a severe drop of perfusion (to $7-23 \%$ of baseline flow), rather than with age 
itself, the serious perfusion deficit being more frequent among the old rats (Menyhart et al., 2017b). Taken together, we postulate that irrespective of the age-related electrophysiological threshold of SD elicitation (which has proven to be higher at old age), a sudden, large drop of cortical perfusion (i.e. blood flow plunging below 20\%) will inevitably bring on SDs in the old brain, as well as in the young. We also believe that even though it is more difficult to trigger SDs in the aging brain, once SD is elicited during ischemia, it is persistent at old age, and its long duration may indicate metabolic crisis (Farkas and Bari, 2014).

The experimental research conducted so far has thus provided compelling evidence for the impact of age on the susceptibility of the cerebral gray matter to SD (Fig. 1), yet clinical studies are sparse in this regard, probably due to the inherent limitation of having to consider a high number of risk factors other than age. Still, SDs were reported to occur at higher incidence in young compared to older patients who suffered acute brain injury (Fabricius et al., 2006), which observation is in harmony with the experimental results.

In order to understand what cellular pathways may be altered to account for the higher resistance of the old nervous tissue against SD, the determining factors of SD occurrence will be examined next.

\section{Mechanisms behind SD susceptibility}

It has been inferred above that various parameters can be taken to express SD susceptibility, such as (i) the latency of SD occurrence with respect to the stimulus, (ii) the frequency of recurrent SD events, (iii) the rate of SD propagation, (iv) the distance SD covers over its course of propagation, or (v) the duration of ECoG depression. Some of these parameters concern SD initiation (i.e. latency with respect to igniting stimulus), others essentially characterize propagation (i.e. rate and distance covered, duration of ECoG depression), or represent a mixture of both initiation and propagation (i.e. frequency of recurrent SDs). The capability of the nervous tissue to recover from SD may be a relevant factor to consider as well, since delayed repolarization may postpone the occurrence of subsequent SD events, and is expected to reduce SD frequency. The network of astrocytes is thought to contribute to this process. In addition, the buffering capacity of astrocytes may also modulate the delay necessary to reach the neurochemical threshold of SD elicitation. Understanding what mechanisms drive SD initiation, propagation, and repolarization is a first step to appreciate how aging may impact on SD susceptibility.

\subsection{SD elicitation}

In the healthy mammalian brain, extracellular $\mathrm{K}^{+}$concentration is kept close to 3-4 mM, independent of fluctuations in blood serum levels (Somjen, 1979), but local changes in extracellular $\mathrm{K}^{+}$levels do occur following neuronal activity. In the injured brain, the concentration of $\mathrm{K}^{+}(10-15 \mathrm{mM})$ sufficient to induce SD is presumably determined by the balance between $\mathrm{K}^{+}$efflux and the efficacy of $\mathrm{K}^{+}$clearance (Spong et al., 2016). In the context of spontaneous SD occurrence under hypoxia or ischemia, an initial outward $\mathrm{K}^{+}$current - which is also reflected by the slow depolarization preceding SD (Hansen, 1977; Hansen and Zeuthen, 1981) - may be, in part, a key trigger. This progressive accumulation of extracellular $\mathrm{K}^{+}$is thought to build up due to the reduced availability of ATP, which opens ATP-sensitive $\mathrm{K}^{+}$channels to hyperpolarize neurons via $\mathrm{K}^{+}$efflux (Sun and $\mathrm{Hu}, 2010$ ), and, more importantly, reduces the efficiency of neuronal $\mathrm{Na}^{+} / \mathrm{K}^{+}$-ATPase and thus $\mathrm{K}^{+}$reuptake (Hajek et al., 1996). In addition, extracellular levels of $\mathrm{K}^{+}$typical of the ischemic penumbra were shown also to decrease $\mathrm{Na}^{+} / \mathrm{K}^{+}$-ATPase activity by $50 \%$ (Major et al., 2017), therefore $\mathrm{K}^{+}$ itself may amplify its extracellular accumulation. The central role of $\mathrm{Na}^{+} / \mathrm{K}^{+}$-ATPase in SD initiation is clearly supported by the fact that tissue exposure to ouabain, a $\mathrm{Na}^{+} / \mathrm{K}^{+}$-ATPase inhibitor, readily produces SD (Balestrino et al., 1999). Ultimately, the excess $\mathrm{K}^{+}$in the extracellular space amounts to a depolarizing stimulus that, by the gradual shift in membrane potential, opens voltage-gated $\mathrm{Na}^{+}$channels to give way to $\mathrm{Na}^{+}$influx. The experimental elicitation of SD with high concentration $\mathrm{KCl}$ or electrical stimulation takes advantage of the artificial elevation of extracellular $\mathrm{K}^{+}$as well.

When a critical threshold of $\mathrm{K}^{+}$levels is reached, the self-propagating SD cycle takes off and invades neighboring tissue (Grafstein, 1956). However, the critical threshold of $\mathrm{K}^{+}$concentration appears to be subject to other, variable conditions such as the degree of metabolic impairment or the maturation of the brain, and a set threshold value that would be uniformly valid cannot be determined. To illustrate the variability of SD threshold from the perspective of aging, the extracellular concentration of $\mathrm{K}^{+}$sufficient for the spontaneous occurrence of SD due to asphyxia in adult rats (i.e. $>24$ weeks old) ranged around $10-12 \mathrm{mM}$, but was over $20 \mathrm{mM}$ in 12 -week-old rats, and could be as high as $30 \mathrm{mM}$ in 4-day-old pups (Hansen, 1977).

In migraine with aura, the progressive accumulation of $\mathrm{K}^{+}$to high concentration as it takes place in injured tissue is unlikely, therefore an increased sensitivity or hyperexcitability of neurons may stand in the center of SD elicitation (Vinogradova, 2018). In addition, hyperexcitablity may be relevant for ischemia-related SD, as well, since theoretically such a condition may predict SD occurrence at a lower threshold. Neuronal excitability is partly regulated by neuronal voltagedependent $\mathrm{Na}^{+}$(Nav), $\mathrm{K}^{+}(\mathrm{Kv})$, and $\mathrm{Ca}^{2+}$ (Cav) channels (Misonou, 2010). Structural variations, modulation by second messengers and genetic dysfunction of these channels might influence the SD threshold, the latter being implicated, for example, in familial hemiplegic migraine (FHM) type 1 (FHM1) and 3 (FHM3) (Dichgans et al., 2005; van den Maagdenberg et al., 2004). As such, FHM3 has been linked to a mutation of the $\alpha 1$ subunit of Nav 1.1 (encoded by the SNCA1A gene), which elicits neuronal hyperexcitability and might decrease SD threshold (Dichgans et al., 2005). Neuronal hyperexcitability, on the other hand, is prevented by the activation of Kv1 type delayed-rectifier $\mathrm{K}^{+}$channels (Hille, 1992). Interestingly, the selective pharmacological blockade of cerebellar Kv 1.1 and Kv1.2 channels - highly expressed in the cerebellar cortex and Purkinje-cells - was shown to decrease significantly the threshold of spreading acidification and depression in the cerebellar cortex, a phenomenon sharing similarities with SD (Chen et al., 2005). Another type of $\mathrm{Kv}$ channel to suppress neuronal excitability is the Kv7.2 (KCNQ2) ion channel (Wulff and Zhorov, 2008). Kv7.2 agonism was found to decrease the frequency of KCl-induced SDs in a dose-dependent manner, probably by promoting membrane hyperpolarization (Wu et al., 2003). Finally, a dominant-negative mutation in the TWIK-related spinal cord $\mathrm{K}^{+}$(TRESK) channel caused hyperexcitability in trigeminal neurons (Liu et al., 2013), and was linked to migraine with aura (Enyedi et al., 2012), but the contribution of TRESK channels to SD elicitation has not been demonstrated yet.

Neuronal excitability may be modulated by Cav channels as well. FHM1 is characterized by a mutation in the CACNA1A gene encoding the P/Q type $\mathrm{Ca}^{2+}$ channel (van den Maagdenberg et al., 2004), increasing its opening probability. In mice, the gain of function mutation was shown to lower electrical SD threshold in vivo in two different variants, S218L and R192Q, both of which were previously shown to be present in FHM patients. The decreased threshold of SD together with increased neuronal excitability is probably mediated by the higher opening probability of the channels at presynaptic terminals (Tottene et al., 2009).

Taken together, these experimental data suggest, that voltage-gated cation channels that tune neuronal excitability contribute to setting the threshold of SD elicitation.

\subsection{SD evolution and propagation}

Irrespective of whether SD is elicited experimentally or occurs spontaneously, the rapid and marked increase of extracellular $\mathrm{K}^{+}$is a powerful trigger, which depolarizes a critical volume of tissue of about $1 \mathrm{~mm}^{3}$ (Matsuura and Bureš, 1971; Tang et al., 2014). The mass cellular 
depolarization leads then to a near-complete breakdown of neuronal transmembrane potential (Somjen, 2001). More specifically, at any point of the tissue involved in SD, the influx of $\mathrm{Na}^{+}$leads the depolarization, causing a reduction of extracellular $\mathrm{Na}^{+}$concentration from 140 to 150 to $50-70 \mathrm{mM}$, accompanied by a sudden extracellular surge of $\mathrm{K}^{+}$from 3 to 4 to $30-60 \mathrm{Mm}$, a concurrent decrease of extracellular $\mathrm{Ca}^{2+}$ levels from 1 to 1.5 to $0.2-0.8 \mathrm{mM}$ and that of $\mathrm{Cl}^{-}$from 130 to $74 \mathrm{mM}$ (Hansen and Zeuthen, 1981; Pietrobon and Moskowitz, 2014). Intracellular ion concentrations obviously change in the opposite direction.

The channels mediating $\mathrm{K}^{+}$efflux during depolarization are still to be explored, but it is reasonable to suggest that $\mathrm{Kv}$ channels must be involved, because the wide spectrum $\mathrm{K}^{+}$channel blocker tetraethylammonium (a drug that acts on inward rectifying and delayed outward rectifying $\mathrm{K}^{+}$channels) (Cook, 1990); and 4-aminopyridine (a blocker of inward rectifier and A-type $\mathrm{K}^{+}$channels) partially limited $\mathrm{K}^{+}$efflux with SD (Aitken et al., 1991; Somjen, 2001). Recent evidence indicates that large-conductance $\mathrm{Ca}^{2+}$-activated $\mathrm{K}^{+}$(BK) channels contribute to the $\mathrm{K}^{+}$surge with SD (Menyhart et al., 2018), and ATPsensitive $\mathrm{K}^{+}$channels that open under metabolic stress could also be involved (Somjen, 2001). Finally, it is suspected that during hypoxia/ ischemia, massive nonselective $\mathrm{Na}^{+}\left(\mathrm{Ca}^{2+}\right) / \mathrm{K}^{+}$conductances take place with SD, via a yet unidentified channel (Czeh et al., 1992, 1993; Gagolewicz, 2017).

$\mathrm{Ca}^{2+}$ influx with SD may take place through P/Q type Cav channels, which was tested by the application of gabapentine, an anticonvulsant agent, with $\mathrm{P} / \mathrm{Q}$ type $\mathrm{Ca}^{2+}$ channel inhibitory potential. Gabapentin infusion effectively suppressed SD susceptibility in the intact rat cortex (Hoffmann et al., 2010). Likewise, the topical application of the P/Q type Cav channel blocker $\omega$-agatoxin was also found to remarkably reduce the frequency of repetitive SDs (Richter et al., 2002).

In addition to the ion dislocations, interstitial glutamate concentration also increases from 3-3.5 to $10-11 \mu \mathrm{M}$ with SD induced by $\mathrm{KCl}$ in normal rat cortex (Hinzman et al., 2015), or well over $100 \mu \mathrm{M}$ in the striatum during anoxia (Satoh et al., 1999). The accumulation of glutamate may manifest via various pathways. Considering SD triggered experimentally in well-nourished tissue, the first results obtained with mutant $\mathrm{P} / \mathrm{Q}$ type Cav mice suggested that $\mathrm{Ca}^{2+}$ influx with SD through these channels liberates glutamate and contributes to SD evolution (Ayata et al., 2000; Pietrobon and Moskowitz, 2014). Glutamate release with SD later was shown to be linked to presynaptic N-methylD-aspartate (NMDA) receptor-dependent vesicular exocytosis (Zhou et al., 2013). Further, SD has been found to open neuronal pannexin-1 channels (Karatas et al., 2013) that may also mediate the release of glutamate to some extent (Cervetto et al., 2013; Di Cesare Mannelli et al., 2015). Conversely, the pharmacologic inhibition of the P2X7pannexin- $1_{\text {pore }}$ complex suppressed SD, which was suggested to alleviate the release of $\mathrm{K}^{+}$, glutamate and pro-inflammatory cytokines (Chen et al., 2017). Glutamate may also originate from astrocytes through pathological processes, including the possibility that increased extracellular $\mathrm{K}^{+}$concentration reverses excitatory amino acid transporters (EAATs) (Harada et al., 2015; Malarkey and Parpura, 2008; Nicholls and Attwell, 1990).

Once glutamate builds up to high concentrations, it may overstimulate NMDA receptors, thereby deepening the depolarization, and contributing to SD propagation by the further accumulation of $\mathrm{K}^{+}$and glutamate (Harreveld, 1959). Although the NMDA receptor is considered the primary glutamate receptor involved in SD facilitation, some recent studies indicate the role of $\alpha$-amino-3-hydroxy-5-methyl-4isoxazole propionate (AMPA) receptors (Costa et al., 2013) and extrasynaptic NMDA receptors (Hardingham and Bading, 2010) as well. Furthermore, recent investigations have suggested the specific involvement of NMDA receptor subtypes NR2A and NR2B in SD susceptibility (Shatillo et al., 2015; Wang et al., 2012). The involvement of the NMDA receptor in the sustenance of SD is underscored by influential clinical studies disclosing that the administration of ketamine, an
NMDA receptor antagonist to patients of acute brain injury inhibits SD occurrence effectively (Carlson et al., 2018; Sakowitz et al., 2009). Finally, the volatile anesthetics isoflurane and $\mathrm{N}_{2} \mathrm{O}$, which also dampen NMDA receptor-based excitatory neurotransmission, were shown to suppress SD susceptibility in rats (Kudo et al., 2008). It must be noted, however, that SD evolving in response to anoxia/ischemia cannot be blocked by NMDA receptor antagonism, suggesting the negligible involvement of NMDA receptors in SD propagation under severe metabolic stress (Pietrobon and Moskowitz, 2014).

The volume of the extracellular space also adjusts the concentration of substances present in the extracellular fluid. Water passively follows the fluxes of $\mathrm{Na}^{+}$and especially $\mathrm{Cl}^{-}$via $\mathrm{Cl}^{-}$-coupled transporters (Steffensen et al., 2015) to cause swelling of dendrites (termed "dendritic beading") and astrocytes (Risher et al., 2012), with a concomitant shrinkage of the extracellular space. This may increase the concentrations of extracellular $\mathrm{K}^{+}$and glutamate even further (Hansen and Olsen, 1980; Phillips and Nicholson, 1979).

All these results testify that once SD has been elicited, the levels of $\mathrm{K}^{+}$and glutamate, which facilitate SD, progressively rise. The propagation of SD, therefore, is thought to be self-sustained, advocated by the volume transmission of high concentration $\mathrm{K}^{+}$and glutamate generated by SD itself.

\subsection{Recovery from $S D$}

SD is a transient event - although its duration depends on the actual tissue metabolic conditions. The repolarization phase of SD, or recovery of the tissue from SD is mediated by neuronal $\mathrm{Na}^{+} / \mathrm{K}^{+}$-ATPase to serve $\mathrm{K}^{+}$reuptake (Major et al., 2017). Importantly, surplus $\mathrm{K}^{+}$is also removed effectively by astrocytes, utilizing various mechanisms including, for instance, astrocytic $\mathrm{Na}^{+} / \mathrm{K}^{+}$-ATPase, and $\mathrm{K}^{+}$siphoning via Kir 4.1 inwardly rectifying $\mathrm{K}^{+}$channels or water flux mediated through aquaporin- 4 channels (Leis et al., 2005). Similar to $\mathrm{K}^{+}$clearance, glutamate buffering is an equally important factor in SD evolution. Glutamate clearance is mainly mediated by excitatory amino acid transporters 1 and 2 (EAAT1 and EAAT2), which are ion- and voltagedependent, predisposing glutamate reuptake highly susceptible to changes in the ionic composition of the cellular environment and transmembrane potential. For example, EAAT2 is not only co-localized with $\mathrm{Na}^{+} / \mathrm{K}^{+}$-ATPase, but is also highly dependent on the ion gradient it generates (Cholet et al., 2002). Among EAAT subtypes, the glial specific EAAT2 appears to be responsible for more than $90 \%$ of glutamate reuptake in the forebrain (Rimmele and Rosenberg, 2016). The effective involvement of astrocytic buffering in SD management was proven by deleting aquaporin-4 channels, or hindering glutamate clearance. As such, the genetic knock-out of aquaporin- 4 channels in mice decreased the rate of the $\mathrm{K}^{+}$surge and $\mathrm{K}^{+}$reuptake with SD, in association with a slower rate of SD propagation, and lower SD frequency (Yao et al., 2015). On the other hand, EAAT2-mediated glutamate clearance hampered in the absence of the $\alpha 2$ subunit of the astrocytic $\mathrm{Na}^{+} / \mathrm{K}^{+}$ATPase was associated with the facilitation of SD initiation (Capuani et al., 2016). Moreover, mice carrying the genetic knock-in of an astrocytic $\mathrm{Na}^{+} / \mathrm{K}^{+}$ATPase $\alpha 2$ subunit loss-of-function mutation - as it occurs in FHM2 patients - were prone to decreased SD threshold and increased rate of SD propagation (Leo et al., 2011). Conversely, ceftriaxone, one of the $\beta$-lactam antibiotics, stimulated the expression of EAAT2 in astrocytes and concurrently raised SD threshold in FHM2 mutant mice, while inhibition of EAAT2 in wild type mice lowered SD threshold (Capuani et al., 2016). These results collectively suggest that EAAT2 - in tight coupling with $\mathrm{Na}^{+} / \mathrm{K}^{+}$ATPase - is essential for glutamate clearance by astrocytes with $\mathrm{SD}$.

The capacity of astrocytes to remove excessive $\mathrm{K}^{+}$and glutamate might be enhanced by spatial dispersion via the astrocyte syncytium, shown to be effective during physiological neuronal activation (Kofuji and Newman, 2004; Pannasch and Rouach, 2013). Astrocytes (among other cell types) are linked by gap-junctions formed by two 
hemichannels, which consist of pore forming proteins termed connexins. Gap junctions are permeable to small molecules, ions and second messengers, and serve as the structural basis for gliotransmission (Rovegno and Saez, 2018). Gap junctions might be assembled of different connexin isoforms, which determines the role and expression pattern of the pore, placing connexins in the position of indicating intercellular communication. The exact role of connexins in SD initiation and propagation has yet to be explored (Rovegno and Saez, 2018), but the hypothesis may be formulated that the inter-astrocytic movement or redistribution of ions and messengers may facilitate ion or glutamate removal, and thus reduce SD susceptibility. In contrast with this view, exposure to non-specific connexin inhibitors (halothane, octanol and heptanol) blocked SD initiation in the isolated chicken retina (Nedergaard et al., 1995), suggesting that connexin-based channels may enable the intercellular diffusion of ions, and support the propagation of SD. However, much of the work that focused on the involvement of connexins in SD evolution must be interpreted with caution, because the non-selective inhibition of connexins probably impaired inter-neuronal in addition to astrocyte network communication. Also, apart from gap junctions, connexins form unpaired hemichannels, which are large and non-specific pores at the intra- and extracellular interface. Their contribution is often not discriminated at data interpretation (Rovegno and Saez, 2018).

Among the numerous connexin isoforms, connexin- 43 has emerged as a constituent of astrocyte gap junctions. Focusing on the glial syncytium alone, the genetic inactivation of connexin- 43 selective for astrocytes markedly reduced astrocyte network communication, and increased the rate of SD propagation in the hippocampus of adult mice (Theis et al., 2003). This refined approach does support the hypothesis posited above, confirming the ability of intact astrocyte network function to keep SD in check.

A recent study also revealed that tonabersat, a migraine prophylactic drug that inhibits SD (Goadsby et al., 2009) does not only modulate neuron-satellite glia signaling in the trigeminal ganglion by acting on connexin-26 (Damodaram et al., 2009), but also blocks connexin-43 hemichannels in isolated human cerebral microvascular endothelial cells during simulated ischemia (Kim et al., 2017). The collective evidence that a connexin- 43 hemichannel blocker drug inhibits SD may be contradictory to the finding that connexin- 43 deletion in astrocytes facilitates SD, but again, the disparity of model systems (i.e. intact mouse brain vs. endothelial cell culture under simulated ischemia; gap junction vs. hemichannel) may render the satisfactory integration of the existing data challenging.

\section{The impact of aging on the mechanisms involved in SD evolution}

\subsection{The impact of aging on SD elicitation}

The aging brain appears to be increasingly resistant to SD initiation, as indicated by the higher threshold of experimental SD elicitation (Hertelendy et al., 2017; Maslarova et al., 2011; Menyhart et al., 2015). Less is known about the generation of spontaneous SDs, so - aware of the limitations - we infer here that data obtained with experimental SD initiation - whether in the intact or the ischemic gray matter - also applies for spontaneous SDs. The reasons behind the increased threshold of SD elicitation at old age may be manifold. It is conceivable that the stimulus applied is dissipated in the tissue before SD is ignited. Some evidence suggest that the excitability of the aged nervous tissue is lower, because the age-specific increase in the production of reactive oxygen species modifies the operation of the redox-sensitive $\mathrm{K}^{+}$channels (Sesti, 2016) (Fig. 2B). This process may modulate the oligomer formation, permeation and gating properties of $\mathrm{Kv}$ channels and $\mathrm{BK}$ channels (Sesti, 2016), but it remains to be explored whether these changes manifest at the level of SD evolution. Age may also hamper the recruitment of the minimum tissue volume required for SD initiation. Indeed, the wave front of SD in the old cerebral cortex appears to be narrower than in the young, as seen in imaging studies, and possibly reflected by the shorter transient ECoG depression typical of SD (Farkas et al., 2011; Hertelendy et al., 2017; Makra, 2018).

\subsection{The impact of aging on $S D$ evolution and propagation}

In addition to the age-related modification of $\mathrm{Kv}$ and $\mathrm{BK}$ channel function mentioned above (Sesti, 2016), P/Q type Cav channels implicated in SD evolution were shown to be affected by aging, as well. Protein levels of $\mathrm{P} / \mathrm{Q}$ type Cav channels in synaptosomes extracted from the cerebral cortex significantly decreased at preserved synaptic density
A

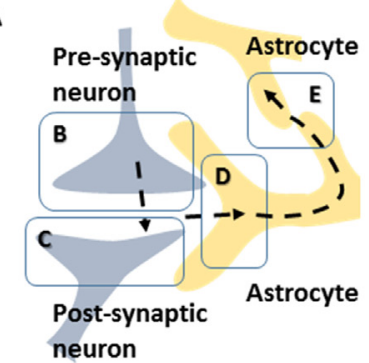

$\begin{array}{lll}\mathrm{E}_{\mathrm{m}} \uparrow \text { Depolarization } & \mathrm{Nav} \\ \mathrm{NMDA} & \mathrm{Kv} \\ \mathrm{Na} / \mathrm{K}^{+} \text {ATPase } & \mathrm{Cav} \\ \text { EAT } & & \begin{array}{l}\text { Gap } \\ \text { junction }\end{array}\end{array}$
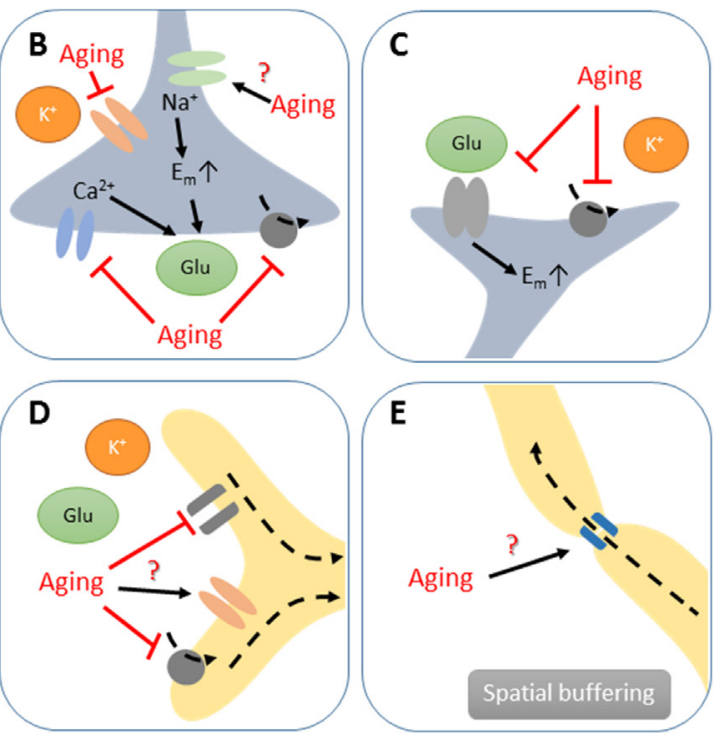

Fig. 2. Conceivable targets of aging, implicated in the susceptibility of the nervous tissue to spreading depolarization (SD). A, Schematic illustration of the cellular elements hosting ion channels and transporters that are involved in SD evolution. B, Agerelated dysfunction of the $\mathrm{Na}^{+} / \mathrm{K}^{+}$ATPase may contribute to $\mathrm{K}^{+}$accumulation in the interstitial space to trigger SD. While little is certain about the impact of aging on voltage-gated $\mathrm{Na}^{+}$channels (Nav), the augmented production of reactive oxygen species with aging may alter voltage-gated $\mathrm{K}^{+}$ channel (Kv) function to reduce the excitability of the nervous tissue (Sesti et al., 2016). Finally, aging may suppress the expression of $\mathrm{P} / \mathrm{Q}$ type voltagegated $\mathrm{Ca}^{2+}$ channels (Cav) (Iwamoto et al., 2004) that may increase SD threshold. C, The age-related downregulation of NMDA receptor subunits implicated in SD (Kumar et al., 2015; Shatillo et al., 2015; Wang et al., 2012) may impair NMDA-based glutamate (Glu) signaling and SD propagation. Potassium reuptake may be also hampered by the activity of the $\mathrm{Na}^{+} / \mathrm{K}^{+}$ATPase decreased in the aging brain (Benzi et al., 1994; Chakraborty et al., 2003;

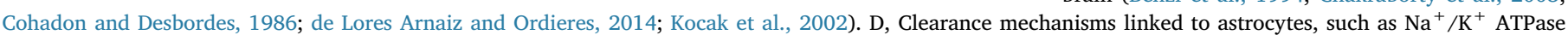

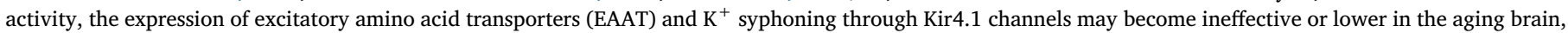

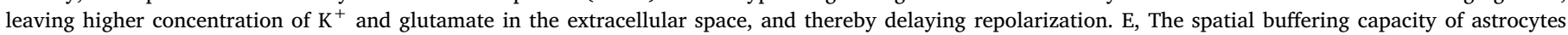

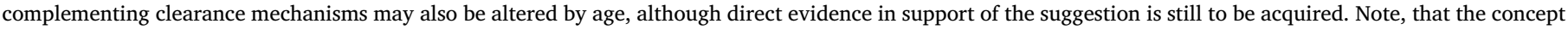
put forward here is not meant to be comprehensive, and may provoke further thoughts. 
in old rats compared to adults (Iwamoto et al., 2004). Since the pharmacological inhibition of $\mathrm{P} / \mathrm{Q}$ type Cav channels was shown to suppress SD occurrence (Richter et al., 2002; Hoffmann et al., 2010), the decreased expression of the channel protein at old age may also impede SD evolution (Fig. 2B).

The ionic movements underlying SD are also accompanied by the release of glutamate, which sustains SD by binding to and activating NMDA receptors. The decay of NMDA receptor-based signaling with aging has been repeatedly demonstrated in the context of suboptimal synaptic neurotransmission and failing cognitive performance (Kumar, 2015). It is plausible that NMDA receptor-based signaling is compromised due to oxidative stress mounting to levels relevant for functional deterioration in the aged brain. Declining NMDA receptor function was, for example, linked to the oxidation of $\mathrm{Ca}^{2+}$ /calmodulin-dependent protein kinase II (Bodhinathan et al., 2010). Among a number of potential mechanisms that may affect NMDA receptors in aging, the expression of distinct NMDA receptor subunits was found to be subject to age-related changes, as well (Kumar, 2015). Importantly, mRNA and protein expression of the modulatory NR2A and the NR2B subunits, both implicated in SD evolution (Shatillo et al., 2015; Wang et al., 2012), appeared to be downregulated in the aged brain (Kumar, 2015; Magnusson et al., 2010; Zhao et al., 2009). In summary, the age-related dysfunction of NMDA receptors may restrict SD evolution (Fig. 2C).

\subsection{The impact of aging on the recovery from $S D$}

The proper function of neuronal and astrocytic $\mathrm{Na}^{+} / \mathrm{K}^{+}$ATPase, and $\mathrm{K}^{+}$and glutamate clearance mechanisms of astrocytes significantly contribute to the cessation of SD (Leis et al., 2005; Major et al., 2017), which is hampered in the old ischemic brain with respect to the young (Clark et al., 2014; Menyhart et al., 2015).

Most studies conducted in this regard agree that the activity of the $\mathrm{Na}^{+} / \mathrm{K}^{+}$-ATPase decreases in the aging brain (Benzi et al., 1994; Chakraborty et al., 2003; Cohadon and Desbordes, 1986; de Lores Arnaiz and Ordieres, 2014; Kocak et al., 2002). Crude miscrosomal preparations must have provided evidence for the combined weakening of neuronal and astrocytic $\mathrm{Na}^{+} / \mathrm{K}^{+}$-ATPase activity, because this approach is not expected to discriminate between cell types (Kocak et al., 2002). On the other hand, investigations using synaptosomes demonstrated the age-related decline in neuronal $\mathrm{Na}^{+} / \mathrm{K}^{+}$-ATPase activity, selectively (Benzi et al., 1994; Chakraborty et al., 2003). Moreover, enzyme activity decreasing with age has been linked to oxidative stress, which is enhanced in the aged brain (Chakraborty et al., 2003). It is noteworthy that $\mathrm{Na}^{+} / \mathrm{K}^{+}$-ATPase hyperactivity in response to ischemia is less obvious in the aging than in the adult brain (Villa et al., 2002), in agreement with the slower recovery from SD reported in old rats suffering from cerebral ischemia (Clark et al., 2014; Menyhart et al., 2015).

Apparently, astrocytes in the aged brain display morphological alterations as well as functional adaptation (Verkhratsky et al., 2016), and show characteristics of senescence-associated secretory phenotype (Salminen et al., 2011). Intuitively, age-related loss of function should include the reduction of the efficacy of astrocyte transport mechanisms, but there is only few and indirect or conflicting evidence to be lined up. As such, both transcript and protein levels of the Kir4.1 channel and the EAAT2 glutamate transporter were shown to be gradually downregulated in the pericontusional cortical region over 3 days after experimental TBI in mice, which proved to be more pronounced in the old group of animals with respect to adults (Gupta and Prasad, 2013). Yet, in the uninjured cortex of the same mice, aging itself increased Kir4.1 and aquaporin- 4 transcript and protein levels, interpreted as an adaptive response to maintain $\mathrm{K}^{+}$and water homeostasis (Gupta and Kanungo, 2013). Further, aquaporin-4 channels participating in astrocytic $\mathrm{K}^{+}$uptake appeared to be less polarized (i.e. more dispersed) in astrocyte end feet in old mice (Kress et al., 2014). The expression of glial fibrillary acidic protein (GFAP) increases with age (Salminen et al.,
2011), and the expression of EAAT2 has been found to decrease with increasing GFAP content, at least in brain samples of Alzheimer's disease patients (Simpson et al., 2010). It is also intriguing that the amplitude of $\mathrm{Ca}^{2+}$ signaling in astrocytes in response to the activation of their NMDA receptors was found markedly decreased at old age in protoplasmic cells isolated from the rodent cortex (Lalo et al., 2011), which may indicate that aging astrocytes are less responsive to glutamate. Altogether, these data are suggestive that transport and signaling by astrocytes are altered in the old brain (Fig. 2D and E). Yet it remains to be investigated to what degree astrocyte aging contributes to the recovery from SD, delayed in the old cerebral gray matter, especially under ischemic conditions.

Finally, astrocyte network function that relies on gap junctional contacts between adjacent cells may be subject to aging. Even though the level of the most typical astrocytic gap junction protein connexin-43 seems to be maintained into old age in the rodent brain and retina, the number, size and connexin composition of astrocytic gap junction plaques may change (Cotrina et al., 2001; Mansour et al., 2013). Whether these subtle but detectable alterations in gap junction structure contribute to any (mal)adaptation of astrocyte network communication with aging is yet to be examined.

\section{Additional factors that modulate SD susceptibility: relevance for brain injury}

\subsection{Metabolic status of the tissue}

The metabolic status of the tissue has already been alluded to in the previous chapters as an important factor to modulate SD evolution, but its distinct aspects have not been discussed so far. The supply of glucose and oxygen are crucial for the proper working of energy-dependent ion pumps, and thus the effective maintenance of resting membrane potential. The first indication that the restricted availability of energy substrates facilitates, while surplus glucose impedes SD was delivered by creating systemic hypo- and hyperglycemia in rats. Hypoglycemia shortened, while hyperglycemia postponed the onset delay of SD in response to hypoxia initiation (Hansen, 1978). Later, hyperglycemia was also shown to elevate the electric threshold of SD elicitation, and to reduce the frequency of high $\mathrm{K}^{+}$-induced recurrent SDs in normallyperfused tissue (Hoffmann et al., 2013). Hypoglycemia, on the other hand, did not alter SD susceptibility, but prolonged the cumulative duration of recurrent SDs elicited in the otherwise intact cortex (Hoffmann et al., 2013). Interestingly, suppression of glycogen, lactate or glucose utilization in the cerebral cortex of mice reduce SD elicitation threshold, suggesting a significant role for astrocyte-neuron lactate shuttle during SD (Kilic et al., 2018). In conclusion, the unlimited supply of circulating plasma glucose may restrict the repeated occurrence of SD in the intact and ischemic cerebral cortex.

While hyperglycemia thus appeared to suppress SD, hyperoxia did not affect the duration of SD, neither did hypoxia in anesthetized, normotensive rats (Sukhotinsky et al., 2010). Yet, in a model of focal cerebral ischemia, episodic hypoxia or sensory stimulation causing neural activation was associated with the elicitation of spontaneous SDs in the somatosensory cortex (von Bornstadt et al., 2015). The mechanisms suggested to be involved include a worsening mismatch between increasing $\mathrm{O}_{2}$ demand against a reduced $\mathrm{O}_{2}$ supply in ischemic penumbra tissue (von Bornstadt et al., 2015).

Anaerobic metabolism in ischemic tissue produces lactic acidosis (Katsura et al., 1991), which is considerably augmented by SDs emerging (Menyhart et al., 2017b; Selman et al., 2004). It has been long appreciated that mild tissue acidosis suppresses $\mathrm{SD}$, because $\mathrm{pH}$ 6.67-6.97, achieved by $\mathrm{HCl}$ or $\mathrm{NaOH}$ application, the elevation of $\mathrm{pCO}_{2}$ or withdrawal of bicarbonate in the medium of brain slice preparations inhibited SD initiation and reduced the velocity of SD propagation (Tombaugh and Somjen, 1996; Tong and Chesler, 2000). Low pH may restrict SD evolution via NMDA receptor inhibition (Tang et al., 1990) 
or by the adjustment of the conductance and gating properties of Kv, Nav, and Cav channels (Tombaugh and Somjen, 1996). Recent analysis has refined the view that acidosis impedes SD by presenting that lower tissue $\mathrm{pH}$ predicted smaller SD amplitude in the normally perfused cerebral cortex only, while the positive correlation between tissue $\mathrm{pH}$ and SD amplitude was lost under ischemia in anesthetized rats (Menyhart et al., 2017a). In the ischemic tissue, the SD suppressing effect of tissue acidosis was proposed to be obscured by glutamate and $\mathrm{K}^{+}$, present at high concentration.

\subsection{Neuroinflammation}

Neuroinflammation has been recognized as a central pathogenic component of cerebral ischemia. Microglia, the resident immune cells of the brain are heavily implicated in mediating the inflammatory response. SD is known to activate microglia (Shibata and Suzuki, 2017), and evidence has also been accumulating recently that activated microglia may, in turn, promote SD generation. Of note, the selective elimination of microglia was found to elevate SD threshold in brain slices (Pusic et al., 2014), and to reduce the incidence of spontaneous SDs in mice subjected to focal cerebral ischemia (Szalay et al., 2016). Particularly, the M1 polarization of microglia may contribute to SD initiation (Pusic et al., 2014). Activated microglia are the source of proinflammatory cytokines such as tumor necrosis factor- $\alpha$ (TNF $\alpha$ ) and interleukin-1 $\beta$ (IL-1 $\beta$ ), which may mediate the microglia-related potentiation of SD. Experimental data addressing the role of TNF $\alpha$ in SD susceptibility remain, however, inconclusive. Exposing brain slices to TNF $\alpha$ for 3 days prior to SD elicitation decreased the threshold of SD (Grinberg et al., 2013), yet the acute, topical administration of TNFa on the cortical surface of anesthetized rodents reduced SD amplitude, probably by augmenting GABA release via the activation of TNF $\alpha$ receptor type-2 (Richter et al., 2014). The action of IL-1 $\beta$ in a similar experimental setting was found dose dependent, as only the lower dose used attenuated SD amplitude (Richter et al., 2017). Microglia-derived cytokines may, therefore, modulate SD susceptibility in various, complex ways, which deserve further examination.

\subsection{Nitric oxide}

Acute cerebral ischemia gives rise to nitric oxide (NO) production, partially in response to the release of pro-inflammatory cytokines (Willmot et al., 2005; Murphy and Gibson, 2007). While NO produced by the neuronal and inducible isoforms of nitric oxide synthase (nNOS and iNOS, respectively) were shown to be neurotoxic (Huang et al., 1994; Zhao et al., 2000), NO from endothelial source (eNOS) may be protective against injury (Huang et al., 1996). From the perspective of SD susceptibility, the non-selective pharmacological blockade of NOS lowered SD threshold (Petzold et al., 2008), while the selective inhibition of nNOS did not significantly alter SD initiation (Petzold et al., 2008; Urenjak and Obrenovitch, 2000). Complementary experiments relying on the use of eNOS or nNOS knock-out mice revealed that particularly eNOS-derived NO upheld the physiological threshold of SD elicitation. The availability of NO from an endothelial source may, therefore, fine-tune the susceptibility of injured tissue to SD.

All these data attest that the network of pathways that are capable of the modulation of SD susceptibility intersect and are rather complex. Obviously, a therapeutical approach to lessen SD occurrence in patients may be most effective if a number of SD suppressing approaches were to be combined and tailored to the unique conditions of specific groups of patients.

\section{Conclusions}

The last fifteen years witnessed a rapid advance in our understanding of the pathophysiologic role SD plays in migraine with aura (Goadsby et al., 2017), and particularly in the progression of secondary lesions in acute brain injury (Dreier et al., 2017; Hartings et al., 2017). Recurrent SDs evolving from minutes up to weeks following the primary insult have been recognized to contribute to the growth of secondary injury of ischemic nature, and worsen clinical outcome of neurological conditions.

Even though aging may reduce the susceptibility of the nervous tissue to SD (Clark et al., 2014; Farkas et al., 2011; Hertelendy et al., 2017; Maslarova et al., 2011; Menyhart et al., 2015), the consequences of SD recurrence in the old brain have proven to be graver (Farkas and Bari, 2014). For example, tissue acidosis implicated in ischemic neurodegeneration is associated with SD, and is disproportionately more pronounced in the old brain than in the young (Menyhart et al., 2017a, 2017b). The cerebral blood flow response to SD may turn into deleterious spreading ischemia in the injured tissue (Dreier, 2011), which is more probable in the aged cerebral cortex than in the young (Clark et al., 2014; Menyhart et al., 2015). In fact, SDs coupled with spreading ischemia appear to cause an overall reduction of cerebral blood flow in the old brain, over a period when partial flow compensation should take place (Clark et al., 2014; Menyhart et al., 2015). Finally, prolonged SD indicative of scarcer metabolic resources of the tissue covers larger tissue volume in the old as compared with the young cerebral cortex in experimental focal ischemia (Clark et al., 2014). All these results underscore the augmented pathogenic potential of SD in the aging brain.

The suppression of recurrent SDs or counteracting SD associated spreading ischemia is a realistic approach for neuroprotection, and is expected to be beneficial for injury outcome after SAH, malignant ischemic stroke or TBI. A number of experimental studies conducted to this end have presented evidence that NMDA receptor blockade (Sanchez-Porras et al., 2015; Reinhart and Shuttleworth, 2018), or the inhibition of $\mathrm{P} / \mathrm{Q}$ type $\mathrm{Ca}^{2+}$ channels potentially reduces SD susceptibility (Hoffmann et al., 2010). On the other hand, L-type voltage-gated $\mathrm{Ca}^{2+}$ channel antagonism was shown to reverse spreading ischemia to hyperemia (Dreier et al., 1998), and lessen the weight of early hypoperfusion in the full cerebral blood flow response to SD (Menyhart et al., 2018). The first clinical trials to prevent repeated SD occurrence by the application of ketamine are promising (Carlson et al., 2018; Sakowitz et al., 2009). Taken that recurrent SDs may pose an increased burden in the aging injured brain, the benefit of therapeutic approaches to restrict SD generation and propagation may be particularly relevant for elderly patients.

\section{Funding}

This work was supported by grants from the National Research, Development and Innovation Office of Hungary (Grant No. K111923 and K120358); the Economic Development and Innovation Operational Programme in Hungary co-financed by the European Union and the European Regional Development Fund (No. GINOP-2.3.2-15-201600006); and the EU-funded Hungarian grant No. EFOP-3.6.1-16- 201600008.

\section{Conflicts of interest}

The Authors declare no conflict of interest.

\section{Appendix A. Supplementary data}

Supplementary data to this article can be found online at https:// doi.org/10.1016/j.neuint.2018.10.010.

\section{References}

Aitken, P.G., Jing, J., Young, J., Somjen, G.G., 1991. Ion channel involvement in hypoxiainduced spreading depression in hippocampal slices. Brain Res. 541, 7-11.

Ayata, C., 2009. Spreading depression: from serendipity to targeted therapy in migraine prophylaxis. Cephalalgia 29, 1095-1114. https://doi.org/10.1111/j.1468-2982. 
2009.01982.x.

Ayata, C., 2010. Cortical spreading depression triggers migraine attack: pro. Headache 50, 725-730. https://doi.org/10.1111/j.1526-4610.2010.01647.x.

Ayata, C., Lauritzen, M., 2015. Spreading depression, spreading depolarizations, and the cerebral vasculature. Physiol. Rev. 95, 953-993. https://doi.org/10.1152/physrev. 00027.2014.

Ayata, C., Shimizu-Sasamata, M., Lo, E.H., Noebels, J.L., Moskowitz, M.A., 2000. Impaired neurotransmitter release and elevated threshold for cortical spreading depression in mice with mutations in the alpha1A subunit of $\mathrm{P} / \mathrm{Q}$ type calcium channels. Neuroscience 95, 639-645.

Back, T., Ginsberg, M.D., Dietrich, W.D., Watson, B.D., 1996. Induction of spreading depression in the ischemic hemisphere following experimental middle cerebral artery occlusion: effect on infarct morphology. J. Cerebr. Blood Flow Metabol. 16, 202-213. https://doi.org/10.1097/00004647-199603000-00004.

Back, T., Kohno, K., Hossmann, K.A., 1994. Cortical negative DC deflections following middle cerebral artery occlusion and $\mathrm{KCl}$-induced spreading depression: effect on blood flow, tissue oxygenation, and electroencephalogram. J. Cerebr. Blood Flow Metabol. 14, 12-19. https://doi.org/10.1038/jcbfm.1994.3.

Balestrino, M., Young, J., Aitken, P., 1999. Block of $(\mathrm{Na}+, \mathrm{K}+)$ ATPase with ouabain induces spreading depression-like depolarization in hippocampal slices. Brain Res. 838, 37-44.

Benzi, G., Gorini, A., Arnaboldi, R., Ghigini, B., Villa, R.F., 1994. Age-related alterations by chronic intermittent hypoxia on cerebral synaptosomal ATPase activities. J. Neural. Transm. Suppl. 44, 159-171.

Bere, Z., Obrenovitch, T.P., Kozak, G., Bari, F., Farkas, E., 2014. Imaging reveals the focal area of spreading depolarizations and a variety of hemodynamic responses in a rat microembolic stroke model. J. Cerebr. Blood Flow Metabol. 34, 1695-1705. https:// doi.org/10.1038/jcbfm.2014.136.

Bodhinathan, K., Kumar, A., Foster, T.C., 2010. Intracellular redox state alters NMDA receptor response during aging through $\mathrm{Ca} 2+/$ calmodulin-dependent protein kinase II. J. Neurosci. 30, 1914-1924. https://doi.org/10.1523/JNEUROSCI.5485-09.2010.

Bosche, B., Graf, R., Ernestus, R.I., Dohmen, C., Reithmeier, T., Brinker, G., Strong, A.J., Dreier, J.P., Woitzik, J., Members of the Cooperative Study of Brain Injury, D, 2010. Recurrent spreading depolarizations after subarachnoid hemorrhage decreases oxygen availability in human cerebral cortex. Ann. Neurol. 67, 607-617. https://doi. org/10.1002/ana.21943.

Bruns Jr., J., Hauser, W.A., 2003. The epidemiology of traumatic brain injury: a review. Epilepsia 44 (10), 2-10.

Bures, J., 1957. The ontogenetic development of steady potential differences in the cer ebral cortex in animals. Electroencephalogr. Clin. Neurophysiol. 9, 121-130.

Busch, E., Gyngell, M.L., Eis, M., Hoehn-Berlage, M., Hossmann, K.A., 1996. Potassiuminduced cortical spreading depressions during focal cerebral ischemia in rats: contribution to lesion growth assessed by diffusion-weighted NMR and biochemical imaging. J. Cerebr. Blood Flow Metabol. 16, 1090-1099. https://doi.org/10.1097/ 00004647-199611000-00002.

Capuani, C., Melone, M., Tottene, A., Bragina, L., Crivellaro, G., Santello, M., Casari, G., Conti, F., Pietrobon, D., 2016. Defective glutamate and K+ clearance by cortical astrocytes in familial hemiplegic migraine type 2. EMBO Mol. Med. 8, 967-986. https://doi.org/10.15252/emmm.201505944.

Carlson, A.P., Abbas, M., Alunday, R.L., Qeadan, F., Shuttleworth, C.W., 2018. Spreading depolarization in acute brain injury inhibited by ketamine: a prospective, randomized, multiple crossover trial. J. Neurosurg. 1-7. https://doi.org/10.3171/2017.12. JNS171665.

Cervetto, C., Alloisio, S., Frattaroli, D., Mazzotta, M.C., Milanese, M., Gavazzo, P., Passalacqua, M., Nobile, M., Maura, G., Marcoli, M., 2013. The P2X7 receptor as a route for non-exocytotic glutamate release: dependence on the carboxyl tail. J. Neurochem. 124, 821-831. https://doi.org/10.1111/jnc.12143.

Chakraborty, H., Sen, P., Sur, A., Chatterjee, U., Chakrabarti, S., 2003. Age-related oxidative inactivation of $\mathrm{Na}+, \mathrm{K}+$-ATPase in rat brain crude synaptosomes. Exp. Gerontol. 38, 705-710. https://doi.org/10.1016/s0531-5565(03)00066-4.

Charpentier, C., Audibert, G., Guillemin, F., Civit, T., Ducrocq, X., Bracard, S., Hepner, H., Picard, L., Laxenaire, M.C., 1999. Multivariate analysis of predictors of cerebral vasospasm occurrence after aneurysmal subarachnoid hemorrhage. Stroke 30, 1402-1408. https://doi.org/10.1161/01.str.30.7.1402.

Chen, G., Gao, W., Reinert, K.C., Popa, L.S., Hendrix, C.M., Ross, M.E., Ebner, T.J., 2005. Involvement of kv1 potassium channels in spreading acidification and depression in the cerebellar cortex. J. Neurophysiol. 94, 1287-1298. https://doi.org/10.1152/jn. 00224.2005 .

Chen, R.L., Balami, J.S., Esiri, M.M., Chen, L.K., Buchan, A.M., 2010. Ischemic stroke in the elderly: an overview of evidence. Nat. Rev. Neurol. 6, 256-265. https://doi.org/ 10.1038/nrneurol.2010.36

Chen, S.P., Qin, T., Seidel, J.L., Zheng, Y., Eikermann, M., Ferrari, M.D., van den Maagdenberg, A., Moskowitz, M.A., Ayata, C., Eikermann-Haerter, K., 2017. Inhibition of the P2X7-PANX1 complex suppresses spreading depolarization and neuroinflammation. Brain 140, 1643-1656. https://doi.org/10.1093/brain/awx085.

Cholet, N., Pellerin, L., Magistretti, P.J., Hamel, E., 2002. Similar perisynaptic glial localization for the $\mathrm{Na}+, \mathrm{K}+$-ATPase alpha 2 subunit and the glutamate transporters GLAST and GLT-1 in the rat somatosensory cortex. Cerebr. Cortex 12, 515-525. https://doi.org/10.1093/cercor/12.5.515.

Clark, D., Institoris, A., Kozak, G., Bere, Z., Tuor, U., Farkas, E., Bari, F., 2014. Impact of aging on spreading depolarizations induced by focal brain ischemia in rats. Neurobiol. Aging 35, 2803-2811. https://doi.org/10.1016/j.neurobiolaging.2014. 06.013.

Cohadon, F., Desbordes, P., 1986. Brain water and aging. Gerontology 32 (1), 46-49. https://doi.org/10.1159/000212827.

Cook, N.S., 1990. Potassium Channels: Structure, Classification, Functional and
Therapeutic Potential. Harwood Halsted Press, Great Britain.

Costa, C., Tozzi, A., Rainero, I., Cupini, L.M., Calabresi, P., Ayata, C., Sarchielli, P., 2013. Cortical spreading depression as a target for anti-migraine agents. J. Headache Pain 14, 62. https://doi.org/10.1186/1129-2377-14-62.

Cotrina, M.L., Gao, Q., Lin, J.H., Nedergaard, M., 2001. Expression and function of astrocytic gap junctions in aging. Brain Res. 901, 55-61.

Crobeddu, E., Mittal, M.K., Dupont, S., Wijdicks, E.F., Lanzino, G., Rabinstein, A.A., 2012 Predicting the lack of development of delayed cerebral ischemia after aneurysmal subarachnoid hemorrhage. Stroke 43, 697-701. https://doi.org/10.1161/ STROKEAHA.111.638403.

Czeh, G., Aitken, P.G., Somjen, G.G., 1992. Whole-cell membrane current and membrane resistance during hypoxic spreading depression. Neuroreport 3, 197-200.

Czeh, G., Aitken, P.G., Somjen, G.G., 1993. Membrane currents in CA1 pyramidal cells during spreading depression (SD) and SD-like hypoxic depolarization. Brain Res. 632, 195-208.

Damodaram, S., Thalakoti, S., Freeman, S.E., Garrett, F.G., Durham, P.L., 2009. Tonabersat inhibits trigeminal ganglion neuronal-satellite glial cell signaling. Headache 49, 5-20. https://doi.org/10.1111/j.1526-4610.2008.01262.x.

de Lores Arnaiz, G.R., Ordieres, M.G., 2014. Brain Na(+), K(+)-ATPase activity in aging and disease. Int. J. Biomed. Sci. 10, 85-102.

de Rooij, N.K., Greving, J.P., Rinkel, G.J., Frijns, C.J., 2013. Early prediction of delayed cerebral ischemia after subarachnoid hemorrhage: development and validation of a practical risk chart. Stroke 44, 1288-1294. https://doi.org/10.1161/STROKEAHA. 113.001125.

Di Cesare Mannelli, L., Marcoli, M., Micheli, L., Zanardelli, M., Maura, G., Ghelardini, C., Cervetto, C., 2015. Oxaliplatin evokes P2X7-dependent glutamate release in the cerebral cortex: a pain mechanism mediated by Pannexin 1. Neuropharmacology 97, 133-141. https://doi.org/10.1016/j.neuropharm.2015.05.037.

Dichgans, M., Freilinger, T., Eckstein, G., Babini, E., Lorenz-Depiereux, B., Biskup, S., Ferrari, M.D., Herzog, J., van den Maagdenberg, A.M.J.M., Pusch, M., Strom, T.M., 2005. Mutation in the neuronal voltage-gated sodium channel SCN1A in familial hemiplegic migraine. Lancet 366, 371-377. https://doi.org/10.1016/s01406736(05)66786-4.

Dijkhuizen, R.M., Beekwilder, J.P., van der Worp, H.B., Berkelbach van der Sprenkel, J.W., Tulleken, K.A.F., Nicolay, K., 1999. Correlation between tissue depolarizations and damage in focal ischemic rat brain1Published on the World Wide Web on 12 July 1999.1. Brain Res. 840, 194-205. https://doi.org/10.1016/s0006-8993(99)01769-2.

Dreier, J.P., 2011. The role of spreading depression, spreading depolarization and spreading ischemia in neurological disease. Nat. Med. 17, 439-447. https://doi.org/ 10.1038/nm.2333.

Dreier, J.P., Fabricius, M., Ayata, C., Sakowitz, O.W., William Shuttleworth, C., Dohmen, C., Graf, R., Vajkoczy, P., Helbok, R., Suzuki, M., Schiefecker, A.J., Major, S., Winkler, M.K., Kang, E.J., Milakara, D., Oliveira-Ferreira, A.I., Reiffurth, C., Revankar, G.S., Sugimoto, K., Dengler, N.F., Hecht, N., Foreman, B., Feyen, B., Kondziella, D., Friberg, C.K., Piilgaard, H., Rosenthal, E.S., Westover, M.B., Maslarova, A., Santos, E., Hertle, D., Sanchez-Porras, R., Jewell, S.L., Balanca, B., Platz, J., Hinzman, J.M., Luckl, J., Schoknecht, K., Scholl, M., Drenckhahn, C., Feuerstein, D., Eriksen, N., Horst, V., Bretz, J.S., Jahnke, P., Scheel, M., Bohner, G., Rostrup, E., Pakkenberg, B. Heinemann, U., Claassen, J., Carlson, A.P., Kowoll, C.M., Lublinsky, S., Chassidim, Y., Shelef, I., Friedman, A., Brinker, G., Reiner, M., Kirov, S.A., Andrew, R.D., Farkas, E., Guresir, E., Vatter, H., Chung, L.S., Brennan, K.C., Lieutaud, T., Marinesco, S., Maas, A.I., Sahuquillo, J., Dahlem, M.A., Richter, F., Herreras, O., Boutelle, M.G., Okonkwo, D.O., Bullock, M.R., Witte, O.W., Martus, P., van den Maagdenberg, A.M., Ferrari, M.D., Dijkhuizen, R.M., Shutter, L.A., Andaluz, N., Schulte, A.P., MacVicar, B., Watanabe, T., Woitzik, J., Lauritzen, M., Strong, A.J., Hartings, J.A., 2017. Recording, analysis, and interpretation of spreading depolarizations in neurointensive care: review and recommendations of the COSBID research group. J. Cerebr. Blood Flow Metabol. 37, 1595-1625. https://doi.org/10.1177/0271678X16654496.

Dreier, J.P., Korner, K., Ebert, N., Gorner, A., Rubin, I., Back, T., Lindauer, U., Wolf, T. Villringer, A., Einhaupl, K.M., Lauritzen, M., Dirnagl, U., 1998. Nitric oxide scavenging by hemoglobin or nitric oxide synthase inhibition by N-nitro-L-arginine induces cortical spreading ischemia when $\mathrm{K}+$ is increased in the subarachnoid space. J. Cerebr. Blood Flow Metabol. 18, 978-990. https://doi.org/10.1097/00004647. 199809000-00007.

Dreier, J.P., Major, S., Manning, A., Woitzik, J., Drenckhahn, C., Steinbrink, J., Tolias, C., Oliveira-Ferreira, A.I., Fabricius, M., Hartings, J.A., Vajkoczy, P., Lauritzen, M. Dirnagl, U., Bohner, G., Strong, A.J., group, C.s., 2009. Cortical spreading ischaemia is a novel process involved in ischaemic damage in patients with aneurysmal subarachnoid haemorrhage. Brain 132, 1866-1881. https://doi.org/10.1093/brain/ awp102.

Dreier, J.P., Reiffurth, C., 2015. The stroke-migraine depolarization continuum. Neuron 86, 902-922. https://doi.org/10.1016/j.neuron.2015.04.004.

Dreier, J.P., Woitzik, J., Fabricius, M., Bhatia, R., Major, S., Drenckhahn, C., Lehmann, T.N., Sarrafzadeh, A., Willumsen, L., Hartings, J.A., Sakowitz, O.W., Seemann, J.H., Thieme, A., Lauritzen, M., Strong, A.J., 2006. Delayed ischaemic neurological deficits after subarachnoid haemorrhage are associated with clusters of spreading depolarizations. Brain 129, 3224-3237. https://doi.org/10.1093/brain/awl297.

Enyedi, P., Braun, G., Czirjak, G., 2012. TRESK: the lone ranger of two-pore domain potassium channels. Mol. Cell. Endocrinol. 353, 75-81. https://doi.org/10.1016/j mce.2011.11.009.

Fabricius, M., Fuhr, S., Bhatia, R., Boutelle, M., Hashemi, P., Strong, A.J., Lauritzen, M., 2006. Cortical spreading depression and peri-infarct depolarization in acutely injured human cerebral cortex. Brain 129, 778-790. https://doi.org/10.1093/brain/awh716.

Farkas, E., Bari, F., 2014. Spreading depolarization in the ischemic brain: does aging have an impact? J Gerontol A Biol Sci Med Sci 69, 1363-1370. https://doi.org/10.1093/ gerona/glu066. 
Farkas, E., Obrenovitch, T.P., Institoris, A., Bari, F., 2011. Effects of early aging and cerebral hypoperfusion on spreading depression in rats. Neurobiol. Aging 32, 1707-1715. https://doi.org/10.1016/j.neurobiolaging.2009.10.002.

Gagolewicz, P.J.A., R.D, 2017. The Elusive Channel Driving Ischemic Spreading Depolarization. Program No. 122.01. Neuroscience Meeting Planner. Society for Neuroscience, Washington, DC 2017. Online.

Goadsby, P.J., Ferrari, M.D., Csanyi, A., Olesen, J., Mills, J.G., Tonabersat, T.O.N.S.G., 2009. Randomized, double-blind, placebo-controlled, proof-of-concept study of the cortical spreading depression inhibiting agent tonabersat in migraine prophylaxis. Cephalalgia 29, 742-750. https://doi.org/10.1111/j.1468-2982.2008.01804.x.

Goadsby, P.J., Holland, P.R., Martins-Oliveira, M., Hoffmann, J., Schankin, C., Akerman, S., 2017. Pathophysiology of migraine: a disorder of sensory processing. Physiol. Rev. 97, 553-622. https://doi.org/10.1152/physrev.00034.2015.

Grafstein, B., 1956. Mechanism of spreading cortical depression. J. Neurophysiol. 19, 154-171. https://doi.org/10.1152/jn.1956.19.2.154.

Grinberg, Y.Y., Dibbern, M.E., Levasseur, V.A., Kraig, R.P., 2013. Insulin-like growth factor-1 abrogates microglial oxidative stress and TNF-alpha responses to spreading depression. J. Neurochem. 126, 662-672. https://doi.org/10.1111/jnc.12267.

Guedes, R.C., Amorim, L.F., Teodosio, N.R., 1996. Effect of aging on cortical spreading depression. Braz. J. Med. Biol. Res. 29, 1407-1412.

Gupta, R.K., Kanungo, M., 2013. Glial molecular alterations with mouse brain development and aging: up-regulation of the Kir4.1 and aquaporin-4. Age 35, 59-67. https:// doi.org/10.1007/s11357-011-9330-5.

Gupta, R.K., Prasad, S., 2013. Early down regulation of the glial Kir4.1 and GLT-1 expression in pericontusional cortex of the old male mice subjected to traumatic brain injury. Biogerontology 14, 531-541. https://doi.org/10.1007/s10522-013-9459-y.

Hadjikhani, N., Sanchez Del Rio, M., Wu, O., Schwartz, D., Bakker, D., Fischl, B., Kwong, K.K., Cutrer, F.M., Rosen, B.R., Tootell, R.B., Sorensen, A.G., Moskowitz, M.A., 2001. Mechanisms of migraine aura revealed by functional MRI in human visual cortex. Proc. Natl. Acad. Sci. U. S. A. 98, 4687-4692. https://doi.org/10.1073/pnas. 071582498.

Hajek, I., Subbarao, K.V., Hertz, L., 1996. Acute and chronic effects of potassium and noradrenaline on $\mathrm{Na}+, \mathrm{K}+$-ATPase activity in cultured mouse neurons and astrocytes. Neurochem. Int. 28, 335-342.

Hansen, A.J., 1977. Extracellular potassium concentration in juvenile and adult rat brain cortex during anoxia. Acta Physiol. Scand. 99, 412-420. https://doi.org/10.1111/j. 1748-1716.1977.tb10394.x.

Hansen, A.J., 1978. The extracellular potassium concentration in brain cortex following ischemia in hypo- and hyperglycemic rats. Acta Physiol. Scand. 102, 324-329. https://doi.org/10.1111/j.1748-1716.1978.tb06079.x.

Hansen, A.J., Olsen, C.E., 1980. Brain extracellular space during spreading depression and ischemia. Acta Physiol. Scand. 108, 355-365. https://doi.org/10.1111/j.1748 1716.1980.tb06544.x.

Hansen, A.J., Zeuthen, T., 1981. Extracellular ion concentrations during spreading depression and ischemia in the rat brain cortex. Acta Physiol. Scand. 113, 437-445. https://doi.org/10.1111/j.1748-1716.1981.tb06920.x.

Harada, K., Kamiya, T., Tsuboi, T., 2015. Gliotransmitter release from astrocytes: functional, developmental, and pathological implications in the brain. Front. Neurosci. 9, 499. https://doi.org/10.3389/fnins.2015.00499.

Hardingham, G.E., Bading, H., 2010. Synaptic versus extrasynaptic NMDA receptor signalling: implications for neurodegenerative disorders. Nat. Rev. Neurosci. 11, 682-696. https://doi.org/10.1038/nrn2911.

Harreveld, A.V., 1959. Compounds in brain extracts causing spreading depression of cerebral cortical activity and contraction of Crustacean muscle. J. Neurochem. 3 300-315. https://doi.org/10.1111/j.1471-4159.1959.tb12636.x.

Hartings, J.A., Bullock, M.R., Okonkwo, D.O., Murray, L.S., Murray, G.D., Fabricius, M. Maas, A.I.R., Woitzik, J., Sakowitz, O., Mathern, B., Roozenbeek, B., Lingsma, H., Dreier, J.P., Puccio, A.M., Shutter, L.A., Pahl, C., Strong, A.J., 2011. Spreading depolarisations and outcome after traumatic brain injury: a prospective observational study. Lancet Neurol. 10, 1058-1064. https://doi.org/10.1016/s1474-4422(11) 70243-5.

Hartings, J.A., Shuttleworth, C.W., Kirov, S.A., Ayata, C., Hinzman, J.M., Foreman, B., Andrew, R.D., Boutelle, M.G., Brennan, K.C., Carlson, A.P., Dahlem, M.A., Drenckhahn, C., Dohmen, C., Fabricius, M., Farkas, E., Feuerstein, D., Graf, R., Helbok, R., Lauritzen, M., Major, S., Oliveira-Ferreira, A.I., Richter, F., Rosenthal, E.S., Sakowitz, O.W., Sanchez-Porras, R., Santos, E., Scholl, M., Strong, A.J., Urbach, A., Westover, M.B., Winkler, M.K., Witte, O.W., Woitzik, J., Dreier, J.P., 2017. The continuum of spreading depolarizations in acute cortical lesion development: examining Leao's legacy. J. Cerebr. Blood Flow Metabol. 37, 1571-1594. https://doi. org/10.1177/0271678X16654495.

Hartings, J.A., Wilson, J.A., Hinzman, J.M., Pollandt, S., Dreier, J.P., DiNapoli, V., Ficker, D.M., Shutter, L.A., Andaluz, N., 2014. Spreading depression in continuous electroencephalography of brain trauma. Ann. Neurol. 76, 681-694. https://doi.org/10 1002/ana.24256.

Hertelendy, P., Menyhart, A., Makra, P., Sule, Z., Kiss, T., Toth, G., Ivankovits-Kiss, O., Bari, F., Farkas, E., 2017. Advancing age and ischemia elevate the electric threshold to elicit spreading depolarization in the cerebral cortex of young adult rats. J. Cerebr. Blood Flow Metabol. 37, 1763-1775. https://doi.org/10.1177/0271678X16643735.

Hertle, D.N., Dreier, J.P., Woitzik, J., Hartings, J.A., Bullock, R., Okonkwo, D.O., Shutter, L.A., Vidgeon, S., Strong, A.J., Kowoll, C., Dohmen, C., Diedler, J., Veltkamp, R., Bruckner, T., Unterberg, A.W., Sakowitz, O.W., Cooperative Study of Brain Injury Depolarizations (COSBID), 2012. Effect of analgesics and sedatives on the occurrence of spreading depolarizations accompanying acute brain injury. Brain 135 (8), 2390-2398.

Hille, B., 1992. Ionic Channels of Excitable Membranes. Sinauer Sunderland, MA.

Hinzman, J.M., Andaluz, N., Shutter, L.A., Okonkwo, D.O., Pahl, C., Strong, A.J., Dreier,
J.P., Hartings, J.A., 2014. Inverse neurovascular coupling to cortical spreading depolarizations in severe brain trauma. Brain 137, 2960-2972. https://doi.org/10. 1093/brain/awu241.

Hinzman, J.M., DiNapoli, V.A., Mahoney, E.J., Gerhardt, G.A., Hartings, J.A., 2015. Spreading depolarizations mediate excitotoxicity in the development of acute cortical lesions. Exp. Neurol. 267, 243-253. https://doi.org/10.1016/j.expneurol.2015.03. 014.

Hoffmann, U., Dilekoz, E., Kudo, C., Ayata, C., 2010. Gabapentin suppresses cortical spreading depression susceptibility. J. Cerebr. Blood Flow Metabol. 30, 1588-1592. https://doi.org/10.1038/jcbfm.2010.92.

Hoffmann, U., Sukhotinsky, I., Eikermann-Haerter, K., Ayata, C., 2013. Glucose modulation of spreading depression susceptibility. J. Cerebr. Blood Flow Metabol. 33, 191-195. https://doi.org/10.1038/jcbfm.2012.132.

Huang, Z., Huang, P.L., Ma, J., Meng, W., Ayata, C., Fishman, M.C., Moskowitz, M.A., 1996. Enlarged infarcts in endothelial nitric oxide synthase knockout mice are attenuated by nitro-L-arginine. J. Cerebr. Blood Flow Metabol. 16, 981-987. https://doi. org/10.1097/00004647-199609000-00023.

Huang, Z., Huang, P.L., Panahian, N., Dalkara, T., Fishman, M.C., Moskowitz, M.A., 1994. Effects of cerebral ischemia in mice deficient in neuronal nitric oxide synthase. Science 265, 1883-1885.

Iwamoto, M., Hagishita, T., Shoji-Kasai, Y., Ando, S., Tanaka, Y., 2004. Age-related changes in the levels of voltage-dependent calcium channels and other synaptic proteins in rat brain cortices. Neurosci. Lett. 366, 277-281. https://doi.org/10.1016/ j.neulet.2004.05.048.

Kao, Y.C., Li, W., Lai, H.Y., Oyarzabal, E.A., Lin, W., Shih, Y.Y., 2014. Dynamic perfusion and diffusion MRI of cortical spreading depolarization in photothrombotic ischemia. Neurobiol. Dis. 71, 131-139. https://doi.org/10.1016/j.nbd.2014.07.005.

Karatas, H., Erdener, S.E., Gursoy-Ozdemir, Y., Lule, S., Eren-Kocak, E., Sen, Z.D., Dalkara, T., 2013. Spreading depression triggers headache by activating neuronal Panx1 channels. Science 339, 1092-1095. https://doi.org/10.1126/science. 1231897.

Katsura, K., Ekholm, A., Asplund, B., Siesjo, B.K., 1991. Extracellular pH in the brain during ischemia: relationship to the severity of lactic acidosis. J. Cerebr. Blood Flow Metabol. 11, 597-599. https://doi.org/10.1038/jcbfm.1991.109.

Kaufmann, D., Theriot, J.J., Zyuzin, J., Service, C.A., Chang, J.C., Tang, Y.T., Bogdanov, V.B., Multon, S., Schoenen, J., Ju, Y.S., Brennan, K.C., 2017. Heterogeneous incidence and propagation of spreading depolarizations. J. Cerebr. Blood Flow Metabol. 37, 1748-1762. https://doi.org/10.1177/0271678X16659496.

Kilic, K., Karatas, H., Donmez-Demir, B., Eren-Kocak, E., Gursoy-Ozdemir, Y., Can, A., Petit, J.M., Magistretti, P.J., Dalkara, T., 2018. Inadequate brain glycogen or sleep increases spreading depression susceptibility. Ann. Neurol. 83, 61-73. https://doi. org/10.1002/ana.25122.

Kim, Y., Griffin, J.M., Nor, M.N.M., Zhang, J., Freestone, P.S., Danesh-Meyer, H.V., Rupenthal, I.D., Acosta, M., Nicholson, L.F.B., O'Carroll, S.J., Green, C.R., 2017. Tonabersat prevents inflammatory damage in the central nervous system by blocking Connexin43 hemichannels. Neurotherapeutics 14, 1148-1165. https://doi.org/10. 1007/s13311-017-0536-9.

Kocak, H., Oner, P., Oztas, B., 2002. Comparison of the activities of $\mathrm{Na}(+), \mathrm{K}(+)$-ATPase in brains of rats at different ages. Gerontology 48, 279-281. https://doi.org/10. $1159 / 000065249$.

Kofuji, P., Newman, E.A., 2004. Potassium buffering in the central nervous system. Neuroscience 129, 1045-1056. https://doi.org/10.1016/j.neuroscience.2004.06. 008.

Kress, B.T., Iliff, J.J., Xia, M., Wang, M., Wei, H.S., Zeppenfeld, D., Xie, L., Kang, H., Xu, Q., Liew, J.A., Plog, B.A., Ding, F., Deane, R., Nedergaard, M., 2014. Impairment of paravascular clearance pathways in the aging brain. Ann. Neurol. 76, 845-861. https://doi.org/10.1002/ana.24271.

Kudo, C., Nozari, A., Moskowitz, M.A., Ayata, C., 2008. The impact of anesthetics and hyperoxia on cortical spreading depression. Exp. Neurol. 212, 201-206. https://doi. org/10.1016/j.expneurol.2008.03.026.

Kumar, A., 2015. NMDA receptor function during senescence: implication on cognitive performance. Front. Neurosci. 9, 473. https://doi.org/10.3389/fnins.2015.00473.

Lalo, U., Palygin, O., North, R.A., Verkhratsky, A., Pankratov, Y., 2011. Age-dependent remodelling of ionotropic signalling in cortical astroglia. Aging Cell 10, 392-402. https://doi.org/10.1111/j.1474-9726.2011.00682.x.

Lauritzen, M., Skyhoj Olsen, T., Lassen, N.A., Paulson, O.B., 1983. Changes in regional cerebral blood flow during the course of classic migraine attacks. Ann. Neurol. 13, 633-641. https://doi.org/10.1002/ana.410130609.

Leão, A.A.P., 1944. Spreading depression of activity in the cerebral cortex. J. Neurophysiol. 7, 359-390. https://doi.org/10.1152/jn.1944.7.6.359.

Leis, J.A., Bekar, L.K., Walz, W., 2005. Potassium homeostasis in the ischemic brain. Glia 50, 407-416. https://doi.org/10.1002/glia.20145.

Leo, L., Gherardini, L., Barone, V., De Fusco, M., Pietrobon, D., Pizzorusso, T., Casari, G., 2011. Increased susceptibility to cortical spreading depression in the mouse model of familial hemiplegic migraine type 2. PLoS Genet. 7https://doi.org/10.1371/journal. pgen.1002129. e1002129.

Liu, F., McCullough, L.D., 2012. Interactions between age, sex, and hormones in experimental ischemic stroke. Neurochem. Int. 61, 1255-1265. https://doi.org/10 1016/j.neuint.2012.10.003.

Liu, P., Xiao, Z., Ren, F., Guo, Z., Chen, Z., Zhao, H., Cao, Y.Q., 2013. Functional analysis of a migraine-associated TRESK K + channel mutation. J. Neurosci. 33, 12810-12824. https://doi.org/10.1523/JNEUROSCI.1237-13.2013.

Magge, S.N., Chen, H.I., Ramakrishna, R., Cen, L., Chen, Z., Elliott, J.P., Winn, H.R., Le Roux, P.D., 2010. Association of a younger age with an increased risk of angiographic and symptomatic vasospasms following subarachnoid hemorrhage. J. Neurosurg. 112, 1208-1215. https://doi.org/10.3171/2009.9.JNS081670. 
Magnusson, K.R., Brim, B.L., Das, S.R., 2010. Selective vulnerabilities of N-methyl-D-aspartate (NMDA) receptors during brain aging. Front. Aging Neurosci. 2, 11. https:// doi.org/10.3389/fnagi.2010.00011.

Major, S., Petzold, G.C., Reiffurth, C., Windmuller, O., Foddis, M., Lindauer, U., Kang, E.J., Dreier, J.P., 2017. A role of the sodium pump in spreading ischemia in rats. J. Cerebr. Blood Flow Metabol. 37, 1687-1705. https://doi.org/10.1177/ $0271678 X 16639059$.

Makra, P.M., Bari, Á., Farkas, E, F., 2018. Spectral and Multifractal Signature of Cortical Spreading Depolarisation in Aged rats., Frontiers in Physiology, Computational Physiology and Medicine Revised Manuscript under Review.

Malarkey, E.B., Parpura, V., 2008. Mechanisms of glutamate release from astrocytes. Neurochem. Int. 52, 142-154. https://doi.org/10.1016/j.neuint.2007.06.005.

Mansour, H., McColm, J.R., Cole, L., Weible 2nd, M., Korlimbinis, A., Chan-Ling, T., 2013. Connexin 30 expression and frequency of connexin heterogeneity in astrocyte gap junction plaques increase with age in the rat retina. PloS One 8https://doi.org/10. 1371/journal.pone.0057038. e57038.

Marrannes, R., Willems, R., De Prins, E., Wauquier, A., 1988. Evidence for a role of the Nmethyl-D-aspartate (NMDA) receptor in cortical spreading depression in the rat. Brain Res. 457 (2), 226-240.

Maslarova, A., Alam, M., Reiffurth, C., Lapilover, E., Gorji, A., Dreier, J.P., 2011. Chronically epileptic human and rat neocortex display a similar resistance against spreading depolarization in vitro. Stroke 42, 2917-2922. https://doi.org/10.1161/ STROKEAHA.111.621581.

Matsuura, T., Bureš, J., 1971. The minimum volume of depolarized neural tissue required for triggering cortical spreading depression in rat. Exp. Brain Res. 12, 238-249. https://doi.org/10.1007/bf00237916.

Mayevsky, A., Doron, A., Manor, T., Meilin, S., Zarchin, N., Ouaknine, G.E., 1996. Cortical spreading depression recorded from the human brain using a multiparametric monitoring system. Brain Res. 740, 268-274. https://doi.org/10.1016/s0006-8993(96) 00874-8.

Mayevsky, A., Manor, T., Meilin, S., Doron, A., Ouaknine, G.E., 1998. Real-time multiparametric monitoring of the injured human cerebral cortex-a new approach. Acta Neurochir. Suppl. 71, 78-81.

Menyhart, A., Farkas, A.E., Varga, D.P., Frank, R., Toth, R., Balint, A.R., Makra, P., Dreier, J.P., Bari, F., Krizbai, I.A., Farkas, E., 2018. Large-conductance $\mathrm{Ca}(2+)$-activated potassium channels are potently involved in the inverse neurovascular response to spreading depolarization. Neurobiol. Dis. 119, 41-52. https://doi.org/10.1016/j. nbd.2018.07.026.

Menyhart, A., Makra, P., Szepes, B.E., Toth, O.M., Hertelendy, P., Bari, F., Farkas, E., 2015. High incidence of adverse cerebral blood flow responses to spreading depolarization in the aged ischemic rat brain. Neurobiol. Aging 36, 3269-3277. https:// doi.org/10.1016/j.neurobiolaging.2015.08.014.

Menyhart, A., Zolei-Szenasi, D., Puskas, T., Makra, P., Bari, F., Farkas, E., 2017a. Age or ischemia uncouples the blood flow response, tissue acidosis, and direct current potential signature of spreading depolarization in the rat brain. Am. J. Physiol. Heart Circ. Physiol. 313, H328-H337. https://doi.org/10.1152/ajpheart.00222.2017.

Menyhart, A., Zolei-Szenasi, D., Puskas, T., Makra, P., Orsolya, M.T., Szepes, B.E., Toth, R., Ivankovits-Kiss, O., Obrenovitch, T.P., Bari, F., Farkas, E., 2017b. Spreading depolarization remarkably exacerbates ischemia-induced tissue acidosis in the young and aged rat brain. Sci. Rep. 7, 1154. https://doi.org/10.1038/s41598-017-01284-4.

Milner, P.M., 1958. Note on a possible correspondence between the scotomas of migraine and spreading depression of Leão. Electroencephalogr. Clin. Neurophysiol. 10, 705. https://doi.org/10.1016/0013-4694(58)90073-7.

Misonou, H., 2010. Homeostatic regulation of neuronal excitability by $\mathrm{K}(+)$ channels in normal and diseased brains. Neuroscientist 16, 51-64. https://doi.org/10.1177/ 1073858409341085.

Murphy, S., Gibson, C.L., 2007. Nitric oxide, ischaemia and brain inflammation. Biochem. Soc. Trans. 35, 1133-1137. https://doi.org/10.1042/BST0351133.

Nedergaard, M., Cooper, A.J., Goldman, S.A., 1995. Gap junctions are required for the propagation of spreading depression. J. Neurobiol. 28, 433-444. https://doi.org/10. 1002/neu.480280404.

Nicholls, D., Attwell, D., 1990. The release and uptake of excitatory amino acids. Trends Pharmacol. Sci. 11, 462-468. https://doi.org/10.1016/0165-6147(90)90129-v.

Pannasch, U., Rouach, N., 2013. Emerging role for astroglial networks in information processing: from synapse to behavior. Trends Neurosci. 36, 405-417. https://doi.org/ 10.1016/j.tins.2013.04.004.

Petzold, G.C., Haack, S., von Bohlen Und Halbach, O., Priller, J., Lehmann, T.N., Heinemann, U., Dirnagl, U., Dreier, J.P., 2008. Nitric oxide modulates spreading depolarization threshold in the human and rodent cortex. Stroke 39, 1292-1299. https://doi.org/10.1161/STROKEAHA.107.500710.

Phillips, J.M., Nicholson, C., 1979. Anion permeability in spreading depression investigated with ion-sensitive microelectrodes. Brain Res. 173, 567-571.

Pietrobon, D., Moskowitz, M.A., 2014. Chaos and commotion in the wake of cortical spreading depression and spreading depolarizations. Nat. Rev. Neurosci. 15, 379-393. https://doi.org/10.1038/nrn3770.

Pinczolits, A., Zdunczyk, A., Dengler, N.F., Hecht, N., Kowoll, C.M., Dohmen, C., Graf, R., Winkler, M.K., Major, S., Hartings, J.A., Dreier, J.P., Vajkoczy, P., Woitzik, J., 2017. Standard-sampling microdialysis and spreading depolarizations in patients with malignant hemispheric stroke. J. Cerebr. Blood Flow Metabol. 37, 1896-1905. https://doi.org/10.1177/0271678X17699629.

Pusic, K.M., Pusic, A.D., Kemme, J., Kraig, R.P., 2014. Spreading depression requires microglia and is decreased by their M2a polarization from environmental enrichment. Glia 62, 1176-1194. https://doi.org/10.1002/glia.22672.

Rabb, C.H., Tang, G., Chin, L.S., Giannotta, S.L., 1994. A statistical analysis of factors related to symptomatic cerebral vasospasm. Acta Neurochir. 127, 27-31.

Reinhart, K.M., Shuttleworth, C.W., 2018. Ketamine reduces deleterious consequences of spreading depolarizations. Exp. Neurol. 305, 121-128. https://doi.org/10.1016/j. expneurol.2018.04.007.

Richter, F., Ebersberger, A., Schaible, H.G., 2002. Blockade of voltage-gated calcium channels in rat inhibits repetitive cortical spreading depression. Neurosci. Lett. 334 (2), 123-126.

Richter, F., Eitner, A., Leuchtweis, J., Bauer, R., Lehmenkuhler, A., Schaible, H.G., 2017. Effects of interleukin-1ss on cortical spreading depolarization and cerebral vasculature. J. Cerebr. Blood Flow Metabol. 37, 1791-1802. https://doi.org/10.1177/ $0271678 X 16641127$.

Richter, F., Lehmenkühler, A., Fechner, R., Manveljan, L., Haschke, W., 1998. Postnatal conditioning for spreading cortical depression in the rat brain. Dev. Brain Res. 106, 217-221. https://doi.org/10.1016/s0165-3806(98)00018-2.

Richter, F., Lutz, W., Eitner, A., Leuchtweis, J., Lehmenkuhler, A., Schaible, H.G., 2014 Tumor necrosis factor reduces the amplitude of rat cortical spreading depression in vivo. Ann. Neurol. 76, 43-53. https://doi.org/10.1002/ana.24176.

Rimmele, T.S., Rosenberg, P.A., 2016. GLT-1: the elusive presynaptic glutamate transporter. Neurochem. Int. 98, 19-28. https://doi.org/10.1016/j.neuint.2016.04.010.

Risher, W.C., Croom, D., Kirov, S.A., 2012. Persistent astroglial swelling accompanies rapid reversible dendritic injury during stroke-induced spreading depolarizations. Glia 60, 1709-1720. https://doi.org/10.1002/glia.22390.

Rovegno, M., Saez, J.C., 2018. Role of astrocyte connexin hemichannels in cortical spreading depression. Biochim. Biophys. Acta 1860, 216-223. https://doi.org/10. 1016/j.bbamem.2017.08.014.

Rowland, M.J., Hadjipavlou, G., Kelly, M., Westbrook, J., Pattinson, K.T., 2012. Delayed cerebral ischaemia after subarachnoid haemorrhage: looking beyond vasospasm. $\mathrm{Br}$. J. Anaesth. 109, 315-329. https://doi.org/10.1093/bja/aes264.

Sakowitz, O.W., Kiening, K.L., Krajewski, K.L., Sarrafzadeh, A.S., Fabricius, M., Strong, A.J., Unterberg, A.W., Dreier, J.P., 2009. Preliminary evidence that ketamine inhibits spreading depolarizations in acute human brain injury. Stroke 40, e519-522. https:// doi.org/10.1161/STROKEAHA.109.549303.

Salminen, A., Ojala, J., Kaarniranta, K., Haapasalo, A., Hiltunen, M., Soininen, H., 2011. Astrocytes in the aging brain express characteristics of senescence-associated secretory phenotype. Eur. J. Neurosci. 34, 3-11. https://doi.org/10.1111/j.1460-9568. 2011.07738.x.

Sanchez-Porras, R., Zheng, Z., Sakowitz, O.W., 2015. Pharmacological modulation of spreading depolarizations. Acta Neurochir. Suppl. 120, 153-157. https://doi.org/10. 1007/978-3-319-04981-6_26.

Satoh, M., Asai, S., Katayama, Y., Kohno, T., Ishikawa, K., 1999. Real-time monitoring of glutamate transmitter release with anoxic depolarization during anoxic insult in rat striatum. Brain Res. 822, 142-148.

Schade, J.P., 1959. Maturational aspects of EEG and of spreading depression in rabbit. J. Neurophysiol. 22, 245-257. https://doi.org/10.1152/jn.1959.22.3.245.

Selman, W.R., Lust, W.D., Pundik, S., Zhou, Y., Ratcheson, R.A., 2004. Compromised metabolic recovery following spontaneous spreading depression in the penumbra Brain Res. 999, 167-174. https://doi.org/10.1016/j.brainres.2003.11.016.

Sengupta, P., 2013. The laboratory rat: relating its age with human's. Int. J. Prev. Med. 4, 624-630.

Sesti, F., 2016. Oxidation of $\mathrm{K}(+)$ channels in aging and neurodegeneration. Aging Dis 7, 130-135. https://doi.org/10.14336/AD.2015.0901.

Shatillo, A., Salo, R.A., Giniatullin, R., Grohn, O.H., 2015. Involvement of NMDA receptor subtypes in cortical spreading depression in rats assessed by fMRI.

Neuropharmacology 93, 164-170. https://doi.org/10.1016/j.neuropharm.2015.01. 028

Shibata, M., Suzuki, N., 2017. Exploring the role of microglia in cortical spreading depression in neurological disease. J. Cerebr. Blood Flow Metabol. 37, 1182-1191. https://doi.org/10.1177/0271678X17690537.

Simpson, J.E., Ince, P.G., Lace, G., Forster, G., Shaw, P.J., Matthews, F., Savva, G., Brayne, C., Wharton, S.B., Function, M.R.C.C., Ageing Neuropathology Study, G, 2010. Astrocyte phenotype in relation to Alzheimer-type pathology in the ageing brain. Neurobiol. Aging 31, 578-590. https://doi.org/10.1016/j.neurobiolaging.2008.05. 015.

Somjen, G.G., 1979. Extracellular potassium in the mammalian central nervous system. Annu. Rev. Physiol. 41, 159-177.

Somjen, G.G., 2001. Mechanisms of spreading depression and hypoxic spreading depression-like depolarization. Physiol. Rev. 81, 1065-1096. https://doi.org/10.1152/ physrev.2001.81.3.1065.

Spong, K.E., Andrew, R.D., Robertson, R.M., 2016. Mechanisms of spreading depolarization in vertebrate and insect central nervous systems. J. Neurophysiol. 116, 1117-1127. https://doi.org/10.1152/jn.00352.2016.

Steffensen, A.B., Sword, J., Croom, D., Kirov, S.A., MacAulay, N., 2015. Chloride cotransporters as a molecular mechanism underlying spreading depolarization-induced dendritic beading. J. Neurosci. 35, 12172-12187. https://doi.org/10.1523/ JNEUROSCI.0400-15.2015.

Strong, A.J., Fabricius, M., Boutelle, M.G., Hibbins, S.J., Hopwood, S.E., Jones, R., Parkin, M.C., Lauritzen, M., 2002. Spreading and synchronous depressions of cortical activity in acutely injured human brain. Stroke 33, 2738-2743. https://doi.org/10.1161/01. str.0000043073.69602.09.

Sukhotinsky, I., Yaseen, M.A., Sakadzic, S., Ruvinskaya, S., Sims, J.R., Boas, D.A., Moskowitz, M.A., Ayata, C., 2010. Perfusion pressure-dependent recovery of cortical spreading depression is independent of tissue oxygenation over a wide physiologic range. J. Cerebr. Blood Flow Metabol. 30, 1168-1177. https://doi.org/10.1038/ jcbfm.2009.285.

Sun, X.L., Hu, G., 2010. ATP-sensitive potassium channels: a promising target for protecting neurovascular unit function in stroke. Clin. Exp. Pharmacol. Physiol. 37, 243-252. https://doi.org/10.1111/j.1440-1681.2009.05190.x.

Szalay, G., Martinecz, B., Lenart, N., Kornyei, Z., Orsolits, B., Judak, L., Csaszar, E., 
Fekete, R., West, B.L., Katona, G., Rozsa, B., Denes, A., 2016. Microglia protect against brain injury and their selective elimination dysregulates neuronal network activity after stroke. Nat. Commun. 7, 11499. https://doi.org/10.1038/ ncomms11499.

Takano, K., Latour, L.L., Formato, J.E., Carano, R.A., Helmer, K.G., Hasegawa, Y., Sotak, C.H., Fisher, M., 1996. The role of spreading depression in focal ischemia evaluated by diffusion mapping. Ann. Neurol. 39, 308-318. https://doi.org/10.1002/ana. 410390307.

Tang, C.M., Dichter, M., Morad, M., 1990. Modulation of the N-methyl-D-aspartate channel by extracellular H + . Proc. Natl. Acad. Sci. Unit. States Am. 87, 6445-6449. https://doi.org/10.1073/pnas.87.16.6445.

Tang, Y.T., Mendez, J.M., Theriot, J.J., Sawant, P.M., Lopez-Valdes, H.E., Ju, Y.S., Brennan, K.C., 2014. Minimum conditions for the induction of cortical spreading depression in brain slices. J. Neurophysiol. 112, 2572-2579. https://doi.org/10. 1152/jn.00205.2014.

Theis, M., Jauch, R., Zhuo, L., Speidel, D., Wallraff, A., Döring, B., Frisch, C., Söhl, G., Teubner, B., Euwens, C., Huston, J., Steinhäuser, C., Messing, A., Heinemann, U., Willecke, K., 2003. Accelerated hippocampal spreading depression and enhanced locomotory activity in mice with astrocyte-directed inactivation of Connexin43. J. Neurosci. 23, 766-776. https://doi.org/10.1523/jneurosci.23-03-00766.2003.

Tombaugh, G.C., Somjen, G.G., 1996. Effects of extracellular pH on voltage-gated Na +, K + and Ca2 + currents in isolated rat CA1 neurons. J. Physiol. 493, 719-732. https:// doi.org/10.1113/jphysiol.1996.sp021417.

Tong, C.K., Chesler, M., 2000. Modulation of spreading depression by changes in extracellular pH. J. Neurophysiol. 84, 2449-2457. https://doi.org/10.1152/jn.2000.84.5. 2449.

Tottene, A., Conti, R., Fabbro, A., Vecchia, D., Shapovalova, M., Santello, M., van den Maagdenberg, A.M., Ferrari, M.D., Pietrobon, D., 2009. Enhanced excitatory transmission at cortical synapses as the basis for facilitated spreading depression in $\mathrm{Ca}(\mathrm{v})$ 2.1 knockin migraine mice. Neuron 61, 762-773. https://doi.org/10.1016/j.neuron. 2009.01.027.

Urenjak, J., Obrenovitch, T.P., 2000. Pharmacological investigation into the involvement of nitric oxide in $\mathrm{K}+$-induced cortical spreading depression. NaunynSchmiedeberg's Arch. Pharmacol. 362, 137-144. https://doi.org/10.1007/ s002100000273.

van den Maagdenberg, A.M., Pietrobon, D., Pizzorusso, T., Kaja, S., Broos, L.A., Cesetti T., van de Ven, R.C., Tottene, A., van der Kaa, J., Plomp, J.J., Frants, R.R., Ferrari, M.D., 2004. A Cacna1a knockin migraine mouse model with increased susceptibility to cortical spreading depression. Neuron 41, 701-710.

Verkhratsky, A., Zorec, R., Rodriguez, J.J., Parpura, V., 2016. Astroglia dynamics in ageing and Alzheimer's disease. Curr. Opin. Pharmacol. 26, 74-79. https://doi.org/ 10.1016/j.coph.2015.09.011.

Victor, T.W., Hu, X., Campbell, J.C., Buse, D.C., Lipton, R.B., 2010. Migraine prevalence by age and sex in the United States: a life-span study. Cephalalgia 30, 1065-1072. https://doi.org/10.1177/0333102409355601.

Villa, R.F., Gorini, A., Hoyer, S., 2002. ATPases of synaptic plasma membranes from hippocampus after ischemia and recovery during ageing. Neurochem. Res. 27, 861-870.

Vinogradova, L.V., 2018. Initiation of spreading depression by synaptic and network hyperactivity: insights into trigger mechanisms of migraine aura. Cephalalgia 38
1177-1187. https://doi.org/10.1177/0333102417724151.

von Bornstadt, D., Houben, T., Seidel, J.L., Zheng, Y., Dilekoz, E., Qin, T., Sandow, N., Kura, S., Eikermann-Haerter, K., Endres, M., Boas, D.A., Moskowitz, M.A., Lo, E.H., Dreier, J.P., Woitzik, J., Sakadzic, S., Ayata, C., 2015. Supply-demand mismatch transients in susceptible peri-infarct hot zones explain the origins of spreading injury depolarizations. Neuron 85, 1117-1131. https://doi.org/10.1016/j.neuron.2015.02. 007.

Wang, M., Chazot, P.L., Ali, S., Duckett, S.F., Obrenovitch, T.P., 2012. Effects of NMDA receptor antagonists with different subtype selectivities on retinal spreading depression. Br. J. Pharmacol. 165, 235-244. https://doi.org/10.1111/j.1476-5381. 2011.01553.x.

Willmot, M., Gray, L., Gibson, C., Murphy, S., Bath, P.M., 2005. A systematic review of nitric oxide donors and L-arginine in experimental stroke; effects on infarct size and cerebral blood flow. Nitric Oxide 12, 141-149. https://doi.org/10.1016/j.niox.2005. 01.003.

Woitzik, J., Dreier, J.P., Hecht, N., Fiss, I., Sandow, N., Major, S, Winkler, M., Dahlem, Y.A., Manville, J., Diepers, M., Muench, E., Kasuya, H., Schmiedek, P., Vajkoczy, P., group, C.s., 2012. Delayed cerebral ischemia and spreading depolarization in absence of angiographic vasospasm after subarachnoid hemorrhage. J. Cerebr. Blood Flow Metabol. 32, 203-212. https://doi.org/10.1038/jcbfm.2011.169.

Woitzik, J., Hecht, N., Pinczolits, A., Sandow, N., Major, S., Winkler, M.K., WeberCarstens, S., Dohmen, C., Graf, R., Strong, A.J., Dreier, J.P., Vajkoczy, P., group, C.s., 2013. Propagation of cortical spreading depolarization in the human cortex after malignant stroke. Neurology 80, 1095-1102. https://doi.org/10.1212/WNL. 0b013e3182886932.

Wu, Y.J., Boissard, C.G., Greco, C., Gribkoff, V.K., Harden, D.G., He, H., L'Heureux, A., Kang, S.H., Kinney, G.G., Knox, R.J., Natale, J., Newton, A.E., Lehtinen-Oboma, S., Sinz, M.W., Sivarao, D.V., Starrett Jr., J.E., Sun, L.Q., Tertyshnikova, S., Thompson, M.W., Weaver, D., Wong, H.S., Zhang, L., Dworetzky, S.I., 2003. (S)-N-[1-(3-morpholin-4-ylphenyl)ethyl]- 3-phenylacrylamide: an orally bioavailable KCNQ2 opener with significant activity in a cortical spreading depression model of migraine. J. Med. Chem. 46, 3197-3200. https://doi.org/10.1021/jm034073f.

Wulff, H., Zhorov, B.S., 2008. K+ channel modulators for the treatment of neurological disorders and autoimmune diseases. Chem. Rev. 108, 1744-1773. https://doi.org/ $10.1021 / \mathrm{cr} 078234 \mathrm{p}$.

Yao, X., Smith, A.J., Jin, B.J., Zador, Z., Manley, G.T., Verkman, A.S., 2015. Aquaporin-4 regulates the velocity and frequency of cortical spreading depression in mice. Glia 63 , 1860-1869. https://doi.org/10.1002/olia.22853.

Zhao, X., Haensel, C., Araki, E., Ross, M.E., Iadecola, C., 2000. Gene-dosing effect and persistence of reduction in ischemic brain injury in mice lacking inducible nitric oxide synthase. Brain Res. 872, 215-218.

Zhao, X., Rosenke, R., Kronemann, D., Brim, B., Das, S.R., Dunah, A.W., Magnusson, K.R., 2009. The effects of aging on N-methyl-D-aspartate receptor subunits in the synaptic membrane and relationships to long-term spatial memory. Neuroscience 162 , 933-945. https://doi.org/10.1016/j.neuroscience.2009.05.018.

Zhou, N., Rungta, R.L., Malik, A., Han, H., Wu, D.C., MacVicar, B.A., 2013. Regenerative glutamate release by presynaptic NMDA receptors contributes to spreading depression. J. Cerebr. Blood Flow Metabol. 33, 1582-1594. https://doi.org/10.1038/jcbfm. 2013.113. 
II. Hertelendy, P., Menyhárt, Á., Makra, P., Süle, Z., Kiss, T., Tóth, G., Ivánkovits-Kiss, O, Bari, F, Farkas, E.

Advancing age and ischemia elevate the electric threshold to elicit spreading depolarization in the cerebral cortex of young adult rats.

J Cereb Blood Flow Metab. 2017 May;37(5):1763-1775. doi:

$10.1177 / 0271678 X 16643735$

IF: $\mathbf{5 . 4 0 7}$ 


\title{
Advancing age and ischemia elevate the electric threshold to elicit spreading depolarization in the cerebral cortex of young adult rats
}

Journal of Cerebral Blood Flow \& Metabolism

20I7, Vol. 37(5) I763-I775

(C) Author(s) 2016

Reprints and permissions:

sagepub.co.uk/journalsPermissions.nav DOI: $10.1177 / 0271678 \times 16643735$ journals.sagepub.com/home/jcbfm

@SAGE

\author{
Péter Hertelendy', Ákos Menyhárt', Péter Makra', \\ Zoltán Süle ${ }^{2}$, Tamás Kiss', Gergely Tóth', \\ Orsolya Ivánkovits-Kiss', Ferenc Bari' and Eszter Farkas'
}

\begin{abstract}
Spreading depolarizations of long cumulative duration have been implicated in lesion development and progression in patients with stroke and traumatic brain injury. Spreading depolarizations evolve less likely in the aged brain, but it remains to be determined at what age the susceptibility to spreading depolarizations starts to decline, especially in ischemia. Spreading depolarizations were triggered by epidural electric stimulation prior and after ischemia induction in the cortex of 7-30 weeks old anesthetized rats $(n=38)$. Cerebral ischemia was achieved by occlusion of both common carotid arteries. Spreading depolarization occurrence was confirmed by the acquisition of DC potential and electrocorticogram. Cerebral blood flow variations were recorded by laser-Doppler flowmetry. Dendritic spine density in the cortex was determined in Golgi-COX stained sections. Spreading depolarization initiation required increasingly greater electric charge with older age, a potential outcome of consolidation of cortical connections, indicated by altered dendritic spine distribution. The threshold of spreading depolarization elicitation increased with ischemia in all age groups, which may be caused by tissue acidosis and increased $\mathrm{K}^{+}$conductance, among other factors. In conclusion, the brain appears to be the most susceptible to spreading depolarizations at adolescent age; therefore, spreading depolarizations may occur in young patients of ischemic or traumatic brain injury at the highest probability.
\end{abstract}

\section{Keywords}

Age, cerebral blood flow, electrophysiology, spreading depolarization, threshold

Received 26 November 2015; Revised 25 January 2016; Accepted 22 February 2016

\section{Introduction}

Spreading depolarization (SD) has been implicated in the progressive expansion of primary lesions and neural injury subsequent to the primary insult in patients of subarachnoid hemorrhage, ischemic stroke, and traumatic brain injury. ${ }^{1-4}$

$\mathrm{SD}$ is an intense, local depolarization of a critical mass of cells, which propagates to adjacent cell populations in the cerebral gray matter by means of increasing extracellular $\mathrm{K}^{+}$or glutamate concentration, involving both neuronal somata and dendritic arborizations. ${ }^{5}$ The dominating element of the cerebral blood flow $(\mathrm{CBF})$ response following $\mathrm{SD}$ in the intact tissue is a remarkable, transient hyperemia; yet in the ischemic brain, the $\mathrm{CBF}$ response may undergo a gradual transformation to uncover ruling vasoconstrictive elements. ${ }^{6}$ This atypical SD-associated CBF variation during ischemia is believed to aggravate the metabolic supply-demand mismatch in the tissue and is thought to mediate the SD-related expansion of ischemic brain injury. ${ }^{4,6,7}$

\footnotetext{
'Department of Medical Physics and Informatics, Faculty of Medicine \& Faculty of Science and Informatics, University of Szeged, Szeged, Hungary ${ }^{2}$ Department of Anatomy, Histology and Embryology, Faculty of Medicine, University of Szeged, Szeged, Hungary
}

\section{Corresponding author:}

Eszter Farkas, Department of Medical Physics and Informatics, Faculty of Medicine, and Faculty of Science and Informatics, University of Szeged, H6720 Szeged, Korányi fasor 9, Hungary. Email: farkas.eszter.I@med.u-szeged.hu 
While the incidence of ischemic stroke and subarachnoid hemorrhage steadily increases with age, ${ }^{8,9}$ traumatic brain injury most often occurs among the young, including children, adolescents and, most of all, young adults. ${ }^{10}$ Because higher SD frequency or longer total SD duration is thought to usher the progress of brain injury during ischemia, ${ }^{11,12}$ it is of importance to determine which age group is the most susceptible for the evolution of SD. This, in turn, could help to identify a segment of the population being at the highest risk for SD-related injury.

The neonatal nervous tissue appears to be too immature to sustain SD; in the intact rat brain, SD can first be induced experimentally between postnatal days $12-15 .^{13}$ On the other hand, the aging nervous tissue proves to be increasingly resistant to experimental SD elicitation, requiring rising concentration of $\mathrm{K}^{+}$ to trigger SD. ${ }^{14,15}$ Similarly, the likelihood of spontaneous SD occurrence in the ischemic rat cortex decreases with age. ${ }^{16}$ It is, however, uncertain at what age the threshold of SD elicitation starts to rise, especially in ischemic tissue.

It is generally assumed that ischemia-induced SDs impose neurodegeneration by the insufficiency of the associated $\mathrm{CBF}$ response, which deprives the tissue at risk of essential nutrients required to maintain the ionic balance across neuronal cell membranes. ${ }^{6}$ We have recently demonstrated that the ischemic cerebral cortex of aged rats - in contrast with young adult ones - is excessively prone to the evolution of inverse neurovascular coupling with $\mathrm{SD},{ }^{15}$ which is possibly responsible for the intensified expansion of ischemic damage in the aged brain. Yet, it remains to be shown whether the kinetics of the SD-associated CBF response varies during young adulthood.

One of the most obvious features of SD propagation is the transient silencing of spontaneous and evoked synaptic activity, seen as a transient depression of the electrocorticogram (ECoG) ${ }^{17} \mathrm{We}$ have previously shown that the SD-related ECoG depression is shortened in 10-month-old as compared with 2-month-old rats but its functional significance remained elusive. ${ }^{18}$

Taken together, here, we set out to determine the electric threshold of SD elicitation under non-ischemic and ischemic condition in young adult rats of increasing age. We also searched for any link between the threshold of SD elicitation and dendritic structure in the cortex. Further, we aimed to determine any potential variation in the SD-related CBF response in association with the advancing age during young adulthood. Finally, we implemented a detailed spectral analysis of the ECoG to identify the specific frequency bands that may be selectively affected by SD or by the progression of lifetime.
Table I. Composition of experimental age groups.

\begin{tabular}{lllll}
\hline $\begin{array}{l}\text { Age of rats } \\
\text { included in the } \\
\text { study (weeks) }\end{array}$ & $\begin{array}{l}\text { Body } \\
\text { weight }(\mathrm{g})\end{array}$ & $n$ & $\begin{array}{l}\text { Age groups defined } \\
\text { for data analysis }\end{array}$ & $n$ \\
\hline 7 & $276 \pm 14$ & 5 & $7-10$ weeks & 21 \\
8 & $332 \pm 11$ & 4 & & \\
9 & $348 \pm 11$ & 5 & & \\
10 & $377 \pm 10$ & 7 & & 12 \\
12 & $403 \pm 19$ & 6 & $12-16$ weeks & \\
16 & $465 \pm 12$ & 6 & & 5 \\
30 & $608 \pm 37$ & 5 & 30 weeks & \\
\hline
\end{tabular}

Note: Data are given as mean \pm stdev.

\section{Materials and methods}

\section{Surgical procedures}

The experimental procedures were approved by the National Food Chain Safety and Animal Health Directorate of Csongrád county, Hungary. The procedures were performed according to the guidelines of the Scientific Committee of Animal Experimentation of the Hungarian Academy of Sciences (updated Law and Regulations on Animal Protection: 40/2013. (II. 14.) Gov. of Hungary), following the EU Directive 2010/ $63 /$ EU on the protection of animals used for scientific purposes and reported in compliance with the ARRIVE guidelines.

Young adult male Sprague-Dawley rats $(n=38)$ of increasing age (Table 1) were used in the study. The animals were purchased from the Charles River Laboratories, Hungary, were group-housed under a normal $12 / 12 \mathrm{~h}$ light/dark cycle, and had free access to food and drinking water. On the day of experiments, the animals were anesthetized with $1.5-2 \%$ isoflurane in $\mathrm{N}_{2} \mathrm{O}: \mathrm{O}_{2}(70 \%: 30 \%)$ and were allowed to breathe spontaneously throughout the experiment. Body temperature was maintained at $37^{\circ} \mathrm{C}$ with a servo-regulated heating pad. Atropine $(0.1 \%, 0.05 \mathrm{ml})$ was administered intramuscularly shortly before surgical procedures to avoid the production of airway mucus. A catheter was inserted into the right femoral artery to monitor the mean arterial blood pressure (MABP) continuously and the arterial blood gas levels before the elicitation of the first SD and before termination of the experiment (i.e., anesthetic overdose).

Next, a midline incision was made in the neck and both common carotid arteries were delicately separated from the surrounding tissue, including the vagal nerves. Lidocain (1\%) was administered topically before opening each tissue layer. A silicone coated fishing line used 
as occluder was looped around each artery for later induction of cerebral ischemia. Rats were transferred to a stereotactic frame and fixed in prone position. Two cranial windows $(\sim 3 \times 3 \mathrm{~mm}) 1 \mathrm{~mm}$ apart were prepared over the right parietal cortex. The bone was carefully thinned using a dental drill (Technobox, Bien Air 810) and gently peeled away to reveal the dura surface. In the caudal window, a small hole was carefully cut on the dura for the positioning of an intracortical microelectrode and an adjacent laser-Doppler probe positioned above the cortical surface.

\section{Recording of electrophysiological variables}

For the concomitant recording of ECoG and slow cortical or direct current (DC) potential, a glass capillary electrode $(20 \mu \mathrm{m}$ outside tip diameter $)$ filled with physiological saline was inserted $800-1000 \mu \mathrm{m}$ deep into the cerebral cortex at the caudal window. $\mathrm{An} \mathrm{Ag} /$ $\mathrm{AgCl}$ reference electrode was implanted under the skin of the animal's neck. DC potential and ECoG were recorded via a high input impedance pre-amplifier (NL102G, NeuroLog System, Digitimer Ltd., United Kingdom), connected to a differential amplifier (NL106, NeuroLog System, Digitimer Ltd., United Kingdom) with associated filter and conditioner systems (NL125, NL530, NeuroLog System, Digitimer Ltd., United Kingdom). Potential line frequency noise $(50 \mathrm{~Hz})$ was removed by a high quality noise eliminator (HumBug, Quest Scientific Instruments Inc., Canada) without any signal attenuation. The resulting signal was digitalized by an analog/digital (A/D) converter (MP150, Biopac Systems Inc., USA) and continuously acquired at a sampling frequency of $1 \mathrm{kHz}$ using the software ACQKNOWLEDGE 4.2.0 (Biopac Systems Inc., USA).

\section{Monitoring local cerebral blood flow}

$\mathrm{SD}$-associated changes in local $\mathrm{CBF}$ were recorded using laser-Doppler flowmetry (LDF). A laser Doppler needle probe (Probe 403 connected to PeriFlux 5000; Perimed AB, Sweden) was positioned right above the cortical surface at the penetration site of the glass capillary electrode with a micromanipulator, avoiding any large pial vessels. The signal was digitized and acquired, together with the DC potential and ECoG, essentially as described above.

\section{Ischemia induction and SD elicitation}

Following a baseline period of over an hour, persistent incomplete global forebrain ischemia was induced by occluding both common carotid arteries permanently ("2-vessel occlusion," 2VO): occluders were pulled on until resistance was felt and then secured in place. Successful ischemia induction was confirmed by a sharp drop of the LDF signal.

A concentric bipolar needle electrode (tip size: $40 \mu \mathrm{m}$, Neuronelektród Kft., Hungary) was placed upon the dura in the rostral window for SD elicitation. It was connected to an opto-coupled stimulus isolator with a constant current output (NL 800, Digitimer Ltd., United Kingdom), a pulse generator (NL301), a with-delay panel (NL405), and a pulse buffer (NL510), which enabled the adjustment of amplitude and duration of the stimuli at will. Stimulation was implemented with a single, cathodal, and rectangular pulse. The charge delivered was quantified as $Q[\mu C]=I[m A] \times t[\mathrm{~ms}]$, and it was raised stepwise with an interstimulus interval of 2 min until SD was observed. Whenever necessary, the position of the needle electrode was adjusted to optimize the contact between the electrode tip and the tissue. Successful elicitation of SD was confirmed by a negative DCshift of an amplitude greater than $5 \mathrm{mV}$ acquired by the recording electrode or by the obvious ECoG depression.

Three SDs were elicited during the baseline period, at an inter-SD interval of at least $20 \mathrm{~min}$. Additional three SDs were generated during ischemia, the first triggered 20-min after ischemia onset or after the spontaneous occurrence of an SD event associated with ischemia induction (Figure 1).

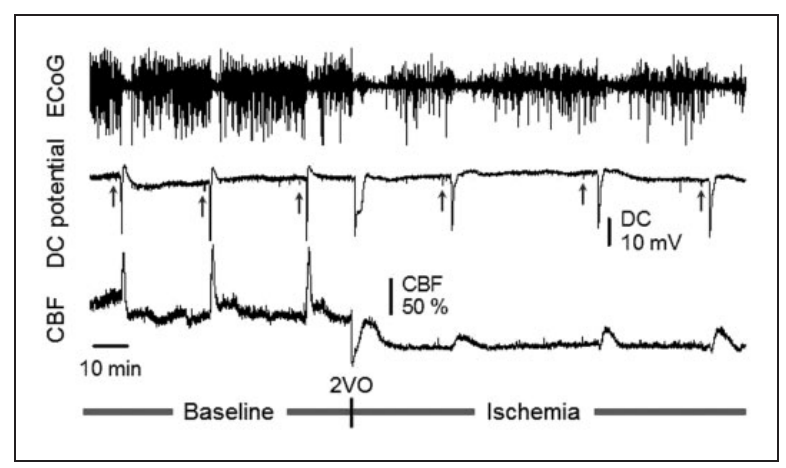

Figure I. Representative, synchronous traces of the ECoG, DC potential, and CBF, acquired from an 8-week-old animal. Depression of the ECoG synchronous with a transient, negative shift of the DC potential and associated functional hyperemia together indicate SD occurrence. The label 2VO (bilateral common carotid artery occlusion, "2-vessel occlusion") designates ischemia induction. Short vertical lines on the DC potential trace show the delivery of current to evoke SD (upward gray arrows). Ischemia induction was immediately followed by the occurrence of a single spontaneous SD. 


\section{Histology}

In order to assess whether dendritic spine density changes with advancing age during young adulthood, additional 8 -week-old $(n=5)$ and 30-week-old $(n=5)$ rats were deeply anesthetized with an overdose of chlorale hydrate (i.p.). The animals were transcardially perfused with ice cold saline, decapitated, and the brains quickly removed. The forebrains were cut in the coronal plane into two pieces of equal size and immersed in Golgi-COX solution mixed of the following stock solutions: (i) $5 \%$ potassium dichromate (Molar Chemicals Kft., Hungary), (ii) 5\% potassium chromate (Molar Chemicals Kft, Hungary), and (iii) $5 \%$ mercuric chloride (VWR International, LLC). ${ }^{19}$ The tissue blocks were incubated for 10 days at room temperature, the solution being refreshed every 2-3 days. The brain samples were then transferred to $30 \%$ sucrose to be stored for at least 10 days at $4{ }^{\circ} \mathrm{C}$. Coronal slices of $200 \mu \mathrm{m}$ were cut with a vibrating microtome, mounted on gelatin-coated microscopic slides, and stored overnight in a dark humidity chamber. Finally, the staining was developed with $30 \%$ ammonium hydroxide followed by Carestream Kodak Fixer (Sigma). Sections were then dehydrated and coverslipped with Eukitt (O. Kindler, Germany).

The stained brain slices were examined by a light microscope (Nikon Eclipse E600). Fifteen-sixteen cortical pyramidal neurons in layer 3 were studied in samples of each animal. Dendritic spine density of second- or third-order dendrites of the proximal apical dendrite was analyzed on a $50-\mu \mathrm{m}$-long segment. Serial z stack images of selected dendritic segments were created using a SPOT RT Slider digital camera $(1600 \times 1200 \mathrm{dpi}$ in 8 bits $)$ connected to the light microscope (Nikon Eclipse E600) and a computer equipped with an image processing software (Image Pro Plus 4.5; Media Cybernetics, Bethesda). Dendritic spines were counted by four independent investigators blind to the experimental groups, using the software IMAGE J (v1.44, National Institute of Health, Bethesda).

\section{Data analysis}

All variables (i.e., DC potential, ECoG, LDF signal and MABP) were simultaneously acquired, displayed live, and stored using a personal computer equipped with a dedicated software (AcqKnowledge 4.2 for MP 150, Biopac Systems, Inc., USA). Data analysis was assisted by the inbuilt tools of the software.

Raw LDF recordings were downsampled to $1 \mathrm{~Hz}$ and then expressed relative to baseline by using the average $\mathrm{CBF}$ value preceding the first evoked SD $(100 \%)$ and the recorded biological zero obtained after terminating the experiment $(0 \%)$ as reference points.
The hyperemic elements of the SD-related CBF response were characterized by the magnitude of hyperemia, which was expressed as peak amplitude with respect to baseline. Hyperemia duration was determined at half amplitude.

ECoG spectral power analysis was applied for individual frequency bands as described previously. ${ }^{15}$ The parameters obtained were the following: (i) level of baseline (the mean spectral power for the 60 -s interval preceding the onset of an SD); (ii) level of depression (the arithmetic mean between the endpoints of the downward and upward segments); (iii) duration of depression; and (iv) level of recovery (the mean spectral power for the 60-s interval following recovery). Along with the 30-week-old group $(n=5)$, six of the youngest animals (7/8 weeks) with clean ECoG trace (i.e., minimal noise) were selected for the analysis.

In order to determine dendritic spine density, the spine count of 15-16 dendritic segments assessed in each animal was averaged, and, thereby, a single value was taken for each rat for further statistical analysis.

Data are given as mean \pm stdev. The software SPSS (IBM SPSS Statistics for Windows, Version 22.0, IBM Corp.) was used for statistical analysis. A one-way analysis of variance (ANOVA) model was used for the evaluation of dendritic spine density, the SD-associated CBF response, baseline ECoG power, and the duration of SD-related ECoG depression. A two-way ANOVA paradigm was applied for the evaluation of data concerning the electric threshold of SD elicitation (factors: age and ischemia) and ECoG power during the SDrelated depression (factors: age and ischemia). A repeated measures ANOVA (factor: age) was used for the analysis of resting $\mathrm{CBF}$, taken at selected time points over the course of experiments, and ECoG power variations over the course of experiments. Wherever appropriate, a Fisher post hoc test was used to reveal the differences between the experimental groups. Pearson two-tailed correlation analysis was carried out to test potential association between data sets. Levels of significance were defined as $p<0.05^{*}$ and $p<0.01 * *$.

\section{Results}

Preliminary screening of the acquired data sets revealed no age-related difference between the 7-10-week-old animals and the 12-16-week-old animals (inserts in Figure 3(a)), therefore, three age groups were created by uniting the 7-10-week-old and the 12-16-week-old populations (Table 1). MABP during baseline was statistically not different across groups $(98 \pm 4,95 \pm 3$, and $107 \pm 10 \mathrm{mmHg}, 7-10$-week-old, 12-16-week-old, and 30-week-old, respectively). Ischemia elevated MABP 


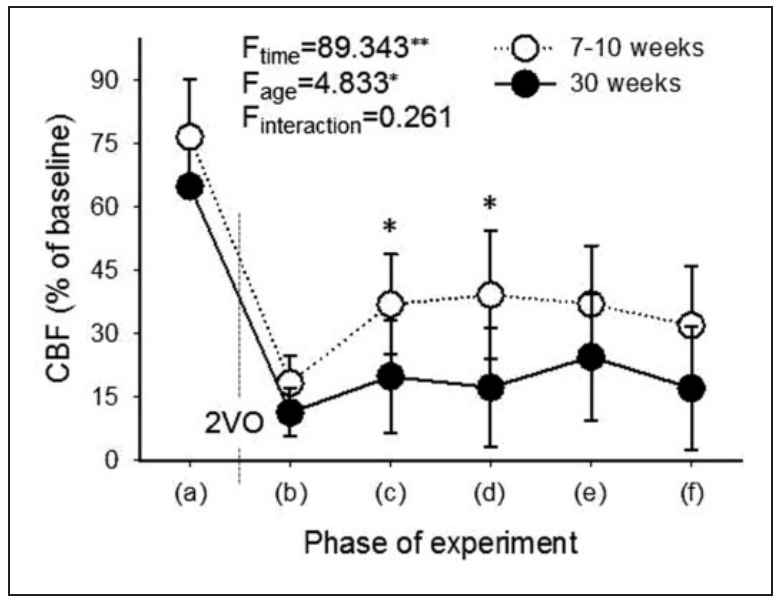

Figure 2. Level of local CBF in the youngest (7-10-week-old) and oldest (30-week-old) age groups, taken at selected time points of the experimental protocol: (a) prior bilateral common carotid artery occlusion (2VO), after the passage of the last SD elicited during baseline; (b) transient drop immediately after 2VO; (c) shortly before the first ischemic SD; (d) shortly before the second ischemic SD; (e) shortly before the third ischemic SD; (f) late ischemia, after the passage of the last SD elicited during ischemia. Data are presented as mean \pm stdev. A repeated measures analysis of variance (ANOVA) paradigm considering age as a factor was applied for statistical analysis. Level of significance was defined as $p<0.01 * *$. Post hoc analysis for group comparison at each time point revealed significant difference at (c) and (d). Level of significance was defined as $p<0.05^{*}$.

slightly but not significantly, without any notable impact of age $(105 \pm 5,96 \pm 4$, and $114 \pm 2 \mathrm{mmHg}$, 710-week-old, 12-16-week-old, and 30-week-old, respectively). Arterial blood gases were typically within the physiological range, with no significant effect of ischemia or age on either $\mathrm{pCO}_{2}$ or $\mathrm{pH}$. Arterial blood glucose concentration taken during baseline and late ischemia was similar (e.g., 9.23 \pm 1.7 vs. $9.20 \pm 1.2 \mathrm{mmol} / 1$, ischemia vs. baseline in 7-10week-old) but significantly increased with advancing age (e.g., $\quad 11.95 \pm 1.6$ vs. $10.08 \pm 1.0$ vs. $9.2 \pm 1.2 \mathrm{mmol} / 1,30$-week-old vs. $12-16$-week-old vs. 7-10-week-old during baseline).

SDs occurred reliably upon electric stimulation of the cortex. In a number of experiments ( 25 of 38 ), a single, spontaneous SD evolved immediately after ischemia induction (Figure 1). The analysis of the DC potential signature of SDs demonstrated that the amplitude of spontaneous SDs $(23.1 \pm 7.8 \mathrm{mV})$ was statistically not different from SDs evoked during baseline $(24.5 \pm 3.8 \mathrm{mV})$ or ischemia $(28.8 \pm 5.8 \mathrm{mV})$. However, with spontaneous SDs, the duration at half amplitude of the DC shift was considerably longer-albeit with excessive variation $(220.9 \pm 316.0 \mathrm{~s})$, as compared with SDs evoked during baseline $(19.9 \pm 5.1 \mathrm{~s})$ or ischemia $(80.7 \pm 80.8 \mathrm{~s})$. Such spontaneous events were not distinctively prevalent for any of the age groups, although only 2 of the 25 events were recorded in the 30 -week-old group. Spontaneous SDs were not included in further data analysis.

The $\mathrm{CBF}$ response to SDs evoked was invariably hyperemic in all age groups under both baseline and ischemia. The $\mathrm{CBF}$ response to the first $\mathrm{SD}$ in each experiment differed from subsequent SDs in that hyperemia was often preceded by a short drop in CBF and was always followed by long-lasting oligemia.

\section{Ischemia evolution and compensation for the reduction of cerebral blood flow after ischemia onset}

In order to estimate how compensation for the reduction of $\mathrm{CBF}$ evolved following ischemia induction, $\mathrm{CBF}$ values were obtained prior to and shortly after ischemia onset, and in between SD events evoked during ischemia (Figure 2). In the youngest, 7-10week-old group, CBF dropped to $18 \pm 6 \%$ immediately after $2 \mathrm{VO}$ onset, recovered to $37 \pm 12 \%$ before the elicitation of the first SD under ischemia, and was maintained over $30 \%$ throughout the ischemic period. In contrast, $\mathrm{CBF}$ fell to $11 \pm 6 \%$ after ischemia induction, recovered to $20 \pm 13 \%$ before initiation of the first ischemic SD, and remained at around only $20 \%$ in the 30 -week-old group, implying significantly less efficient compensation in the 30 -week-old group with respect to the 7-10-week-old group.

\section{Electric threshold of SD elicitation}

The electric threshold of SD elicitation was expressed as the lowest electric charge sufficient to trigger SD in the cortex (Figure 3(a)). We chose to trigger SDs by electric stimulation as opposed to high concentration $\mathrm{K}^{+}$application, because the electric charge delivered can be finely tuned. This offers the accurate estimation of the electric threshold of SD elicitation to uncover any fine variations between age groups of a few weeks difference.

In all age groups, the threshold of SD elicitation was significantly higher during ischemia, as compared with baseline. The initiation of SD required increasingly greater electric charge with older age during both baseline $(4743 \pm 1282$ vs. $3076 \pm 915$ vs. $1661 \pm 649 \mu \mathrm{C}, 30$ week-old vs. 12-16-week-old vs. 7-10-week-old) and ischemia $\quad(8447 \pm 1763 \quad$ vs. $5343 \pm 2170 \quad$ vs. $2514 \pm 1032 \mu \mathrm{C}, 30$-week-old vs. 12-16-week-old vs. 7-10-week-old). Finally, with advancing age, the threshold during ischemia progressively departed from the threshold determined for the respective baseline: in other words, the difference between ischemia and baseline thresholds was approximately five times greater in the 30-week-old as compared with the 7-10-week-old 


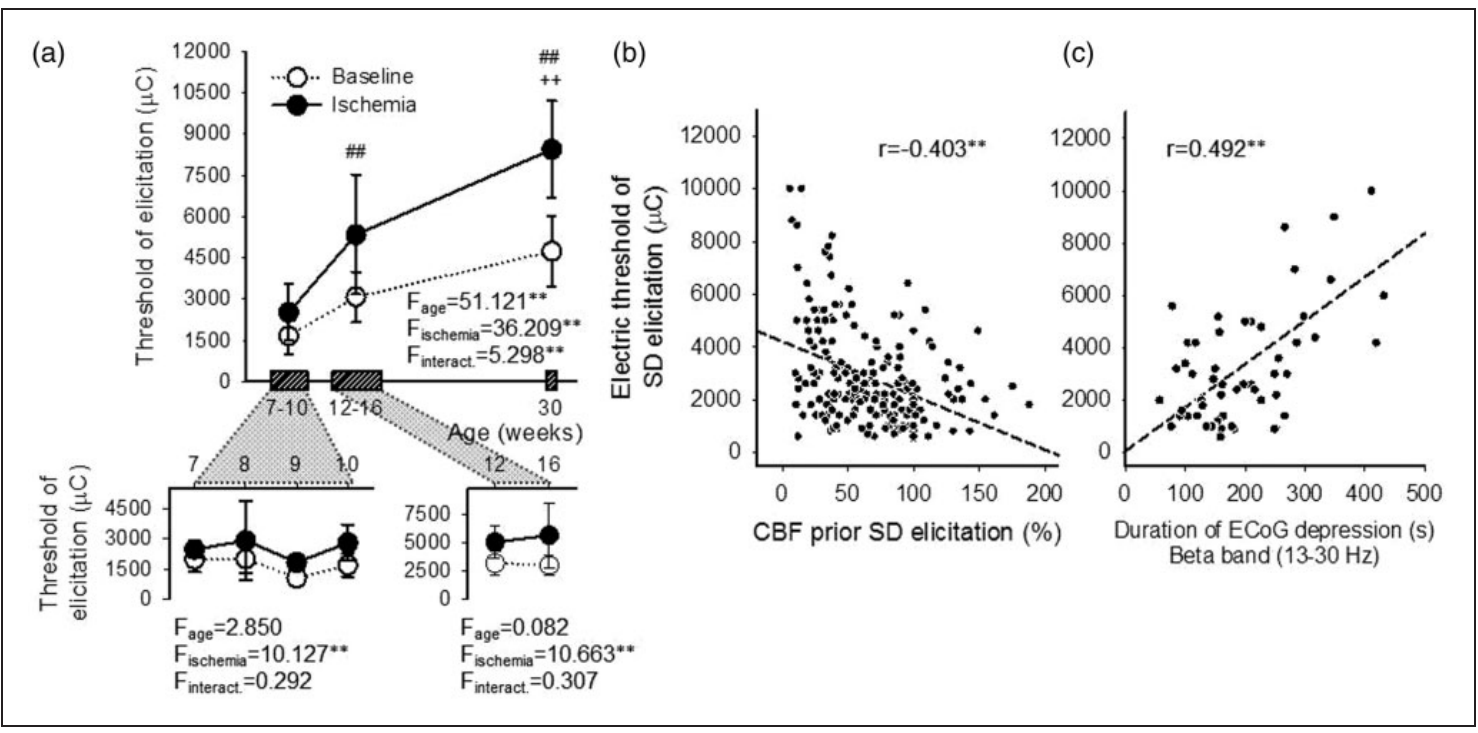

Figure 3. Electric threshold of SD elicitation, and its association with CBF and the SD-related depression of the ECoG. (a) Electric threshold of SD elicitation prior and during incomplete forebrain ischemia (baseline: open symbols, ischemia: closed symbols). The 7I0-week-old animals and I2-16-week-old animals were treated as single groups, because no impact of age was observed on threshold values (see inserts). Data are represented as mean \pm stdev. For the evaluation of statistical significance, a two-way ANOVA paradigm considering age and ischemia as its factors was followed by a Fisher post hoc test for age. Level of significance for the ANOVA was

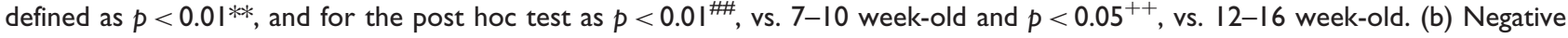
correlation between the electric threshold of SD elicitation and CBF prior SD evolution; each evoked SD was considered for the analysis. Pearson two-tailed correlation analysis including all age groups as one data pool indicated significant association between the threshold and CBF ( $p<0.0$ I** $^{* *}$. (c) Correlation between the electric threshold of SD elicitation and the duration of SD-related depression on the beta frequency band of the ECoG. Data acquired from animals selected for ECoG spectral analysis are shown. Pearson two-tailed correlation analysis revealed significant association between the data sets $\left(p<0.0 \mathrm{I}^{* *}\right)$.

group (threshold difference of $4278 \pm 2352$ vs. $853 \pm 839 \mu \mathrm{C}, 30$-week-old vs. 7-10-week-old).

Next, we set out to determine any potential association between the electric threshold of SD elicitation and $\mathrm{CBF}$ or the cortex' SD-related electrical activity. Lower CBF taken prior SD elicitation predicted higher threshold of SD elicitation $\left(r=-0.403^{* *}\right)$ (Figure 3(b)). The threshold of SD elicitation positively correlated with the duration of SD-related depression of the high frequency alpha and beta bands $\left(r=0.373^{* *}\right.$ and $0.478^{* *}$, respectively) (Figure 3(c)) but not with the low frequency delta and theta bands $(r=0.247$ and 0.234 , respectively). No association with any other ECoG variable (i.e., power of baseline, depression, or recovery) emerged.

\section{Density of dendritic spines}

We set out to test the hypothesis that the increase in the threshold of SD elicitation due to brain maturation is determined - at least in part-by the dynamic structural development of the cortex, reflected by the morphological plasticity of dendritic spines. The animals admitted to the analysis were naive (i.e., neither ischemia nor SD was induced prior to sampling), therefore, potential dendritic spine density changes due to ischemia or SD propagation were not studied.

Dendritic spines on second- or third-order dendritic branches of layer 3 pyramidal neuronal apical dendrites were investigated (Figure 4(a)). In general, dendritic spines tended to be arranged in clusters rather than individually and appeared to be larger and more complex in shape in the 30 -week-old group than in the 8-week-old group (Figure 4(b)). Dendritic spine density significantly increased in the 30 -week-old group with respect to the 8 -week-old group ( $55 \pm 4$ vs. $51 \pm 2$ spines $/ 50 \mu \mathrm{m}$ dendritic segment, 30-week-old vs. 8-week-old) (Figure 4(c)).

\section{Features of the cerebral blood flow response associated with $S D$}

For the evaluation of the SD-associated CBF response, SDs were sorted into three distinct categories: (i) SD1, (ii) subsequent SDs evoked during baseline, and (iii) SDs initiated under ischemia (Figure 5(a)).

As presented in Figure 5(b), CBF prior to the onset of baseline SDs was $10-15 \%$ lower than that prior to SD1 in all age groups (e.g., $90 \pm 30$ vs. $102 \pm 12 \%$, baseline SDs vs. SD1 in 7-10-week-old). The CBF 


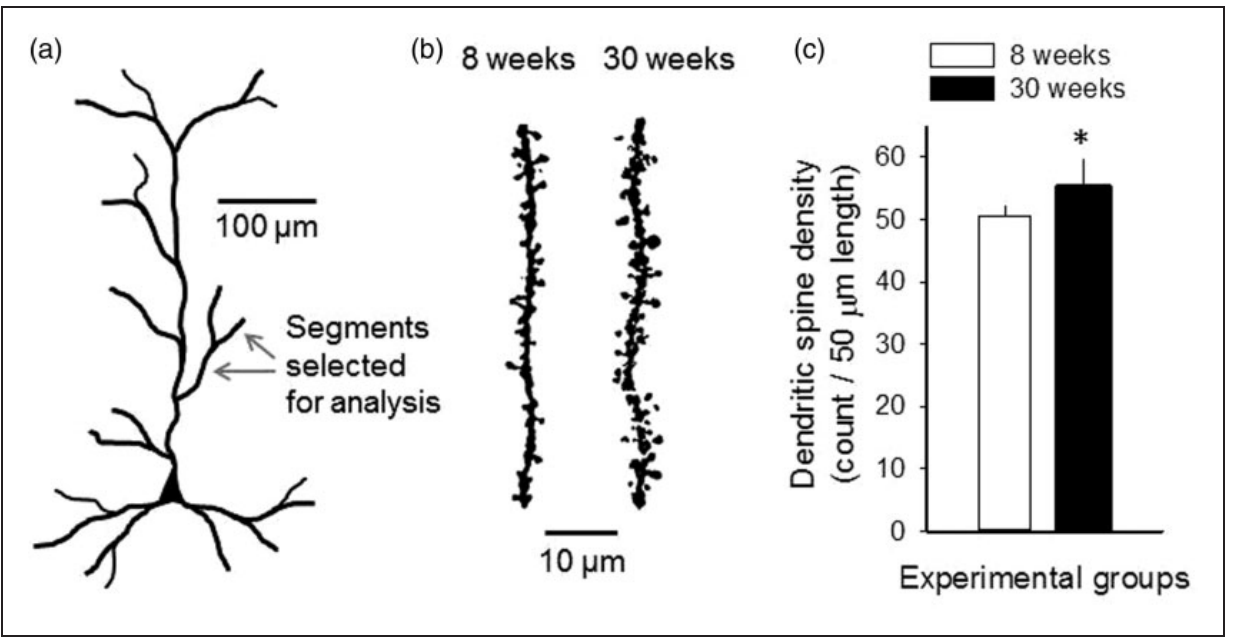

Figure 4. Dendritic spine density on second- or third-order dendritic branches of apical dendrites of layer 3 pyramidal neurons. (a) Schematic drawing of a cortical pyramidal neuron to illustrate the position of dendritic segments investigated. (b) Representative images of 50 - $\mu \mathrm{m}$-long segments of second- or third-order dendrites in the 8-week-old and the 30-week-old groups. Images were obtained of original z-stack images by the enhancement of contrast and brightness in order to eliminate background. (c) Dendritic spine density on the selected dendritic segments. Data are given as mean \pm stdev. A one-way ANOVA model was used for statistical analysis ( $n=5$ for each group, $F=6.263, p<0.05^{*}$ ).

response with SDs under ischemia took off from a markedly lower level $(39 \pm 15$ and $38 \pm 11 \%, 7-10$ week-old, and 12-16-week-old, respectively), especially in the 30 -week-old group $(23 \pm 14 \%)$. The SD-associated hyperemia peaked at around $160-170 \%$ in all age groups during baseline (i.e., $163 \pm 45,160 \pm 61$, and $170 \pm 69 \%, 7-10$-week-old, 12-16-week-old, and 30-week-old, respectively), approximately 20-30\% higher than with SD1 $(129 \pm 23,131 \pm 17$, and $149 \pm 24 \%$, 7-10-week-old, 12-16-week-old, and 30week-old, respectively). During ischemia, the peak amplitude of the CBF response reached a considerably lower level $(59 \pm 23$ and $61 \pm 18 \%$, \%, in 7-10-weekold, and 12-16-week-old, respectively) with the lowest value measured in the 30 -week-old group $(39 \pm 22 \%)$. Conspicuously, in the 30 -week-old group, the CBF response with ischemic SDs set off from the lowest CBF level and reached the lowest peak (not even exceeding the resting $\mathrm{CBF}$ prior to SD elicitation in the 7-10- or the 12-16-week-old groups) (Figure 5(b)), although with no statistically significant reduction with respect to the younger age groups.

Figure 5(c) shows that the duration of the SDrelated $\mathrm{CBF}$ response was shortest with SD1 $(30 \pm 12$, $33 \pm 7$, and $39 \pm 23 \mathrm{~s}, 7-10$-week-old, 12-16-week-old, and 30-week-old, respectively), increased with subsequent SDs elicited during baseline (58 \pm 14 , $56 \pm 11$, and $50 \pm 10 \mathrm{~s}, 7-10$-week-old, 12-16-weekold, and 30-week-old, respectively), and more than doubled with SDs triggered under ischemia (154 \pm 61 , $137 \pm 68$ and $150 \pm 52$ s, 7-10-week-old, 12-16-week-old and 30-week-old, respectively). No impact of age on the duration of the SD-associated $\mathrm{CBF}$ response was observed.

\section{Spectral analysis of the distinct ECoG frequency bands}

Baseline ECoG power was assessed at the very beginning of the experiments, prior to any intervention, to test whether anesthesia affects the two age groups differently. No age-related difference was observed concerning any of the frequency bands studied (Figure 6(a)). ECoG power repeatedly sampled prior to SD events demonstrated that ischemia itself imposed a considerable power decrease on the overall ECoG, which was particularly prominent after the passage of the first SD evoked under ischemia. This is illustrated in Figure 6(b) for the theta band.

The novel findings of spectral analysis concerned the SD-related transient depression of the ECoG. Although the power of the four frequency bands during depression was similar under baseline and ischemia (i.e., ischemia exerted no impact), the selectively greater power of the alpha and theta bands in the 30-week-old group as compared with the 7/8-week-old group (e.g., theta band: $0.005 \pm 0.003$ vs. $0.0025 \pm 0.001 \mathrm{~V}^{2}, 30$-week-old vs. $7 /$ 8 -week-old) indicated incomplete depression of these two bands with the maturation of the brain, under both baseline and ischemic conditions (Figure 6(c)).

As expected, ischemia considerably extended the duration of ECoG depression on all four frequency 


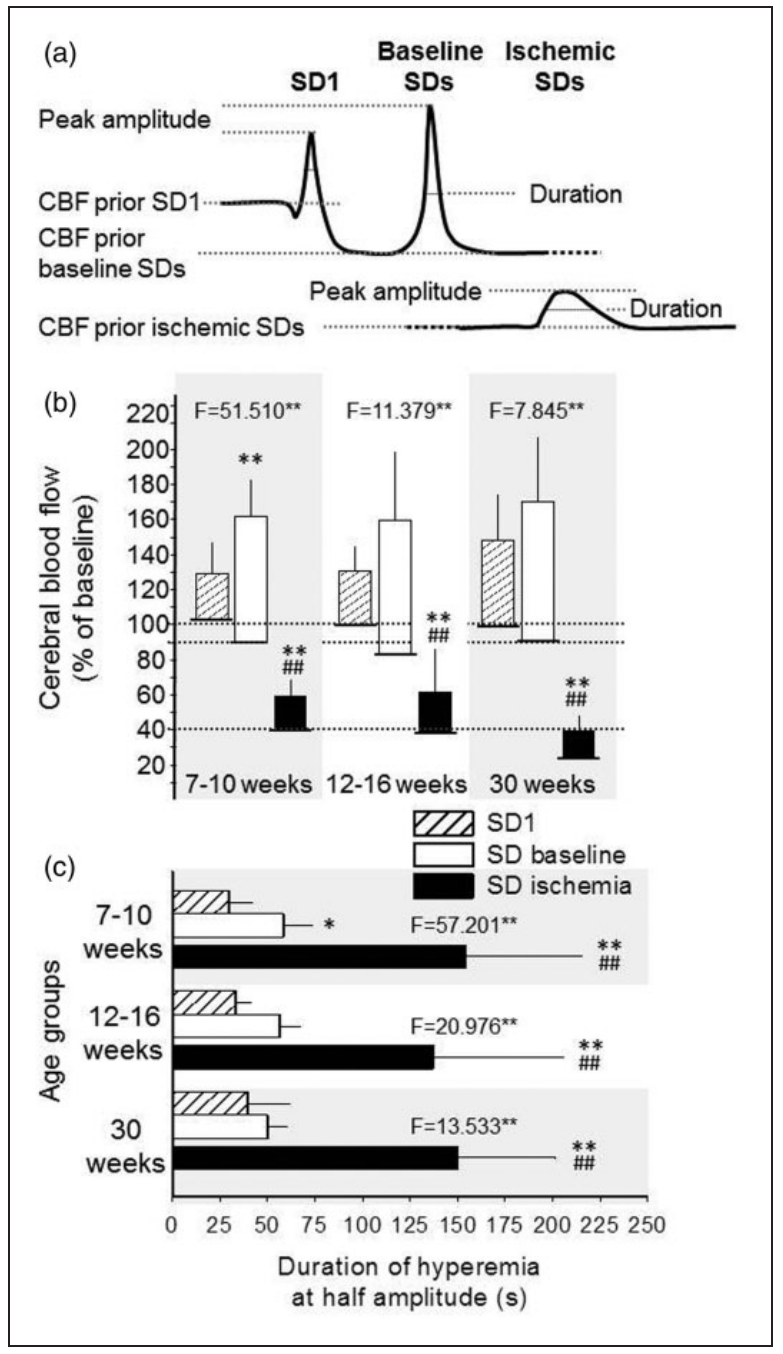

Figure 5. Quantitative analysis of the CBF response associated with SD. (a) Schematic drawings depicting the variables under study: CBF response to SDI, subsequent SDs during baseline (baseline SDs), and SDs evoked during ischemia (ischemic SDs). CBF level prior SD elicitation, peak amplitude, and duration at half amplitude were evaluated. (b) Peak CBF amplitude (bars) respective to CBF baseline prior SD elicitation (base of bars). (c) The duration of hyperemia at half amplitude. Data are expressed as mean \pm stdev. For the evaluation of statistical significance, a one-way ANOVA paradigm followed by a Fisher post hoc test was used for each age group. Level of significance for the ANOVA was defined as $p<0.0 I^{* *}$, and the $F$-values are given in each bar chart. Level of significance for the post hoc analysis is indicated as $p<0.05^{*}$ and $p<0.0 I^{* *}$ vs. SDI; and $p<0.0 I^{\# \#}$ vs. baseline SDs.

bands (e.g., alpha band in the 7/8-week-old: $271 \pm 126$ vs. $139 \pm 40 \mathrm{~s}$, ischemia vs. baseline). Curiously, during baseline, the lower frequency delta and theta bands started to recover from the SD-related depression sooner than the higher frequency alpha and beta bands in the 30 -week-old group, in contrast with the 7/8-week-old group (e.g., duration of theta band: $123 \pm 52$ vs. $132 \pm 34$ s, 30-week-old vs. 7/8-week-old) (Figure 6(d)).

The duration of the SD-related ECoG depression appeared to be associated with the SD-coupled CBF response: the relative peak of $\mathrm{SD}$-associated hyperemia correlated negatively with the duration of ECoG depression on all four frequency bands, as illustrated for the theta band in Figure 6(e) $\left(r=-0.550^{* *}\right)$. At the same time, the longer duration of hyperemia was strongly linked to the longer duration of ECoG depression on all four frequency bands, being most prominent on the delta and theta bands $\left(r=0.733^{*}\right.$ and $r=0.703^{* *}$, respectively) (Figure 6(f)).

\section{Discussion}

\section{Relevance of the findings to human brain injury}

Recurrent SDs spontaneously occur in patients of subarachnoid hemorrhage, ischemic stroke, and traumatic brain injury. ${ }^{1-3}$ The findings of the present study can be interpreted in the context of both ischemic and traumatic brain injury for the following reasons. We studied the brain's susceptibility to SD under two conditions: (i) in the intact and (ii) in the ischemic brain. The results obtained under ischemia are proposed to be relevant to cerebral ischemia evolving in patients of stroke or subarachnoid hemorrhage. The experiments done in the intact brain have revealed that increasing age, by itself, has a significant effect on the elicitation threshold of SD. Thus, the agerelated overall susceptibility of the brain to SD may be a factor to determine the frequency of SD occurrence in any pathophysiological condition, traumatic brain injury being one of them.

In the present study, we relied on electric stimulation to elicit SDs and quantify the electric threshold of SD elicitation with precision. Yet, in the injured brain of patients, SDs occur spontaneously. The exact conditions that give rise to SD generation under ischemia are not absolutely clear but worsening oxygen supply-demand mismatch as a result of somatosensory activation, episodic hypoxemia, or hypotension has been shown to promote SD occurrence. ${ }^{20}$ Taken together, the risk of SD occurrence in the injured brain must be dependent on a combination of several factors, such as the severity of ischemia, insufficiency of oxygen delivery, and, as proposed here, the brain's age-related susceptibility to sustain SD. Our argument is also supported by a previous study of ours showing that spontaneous SDs occur significantly less likely in the aged ischemic brain with respect to the young. ${ }^{16}$ 


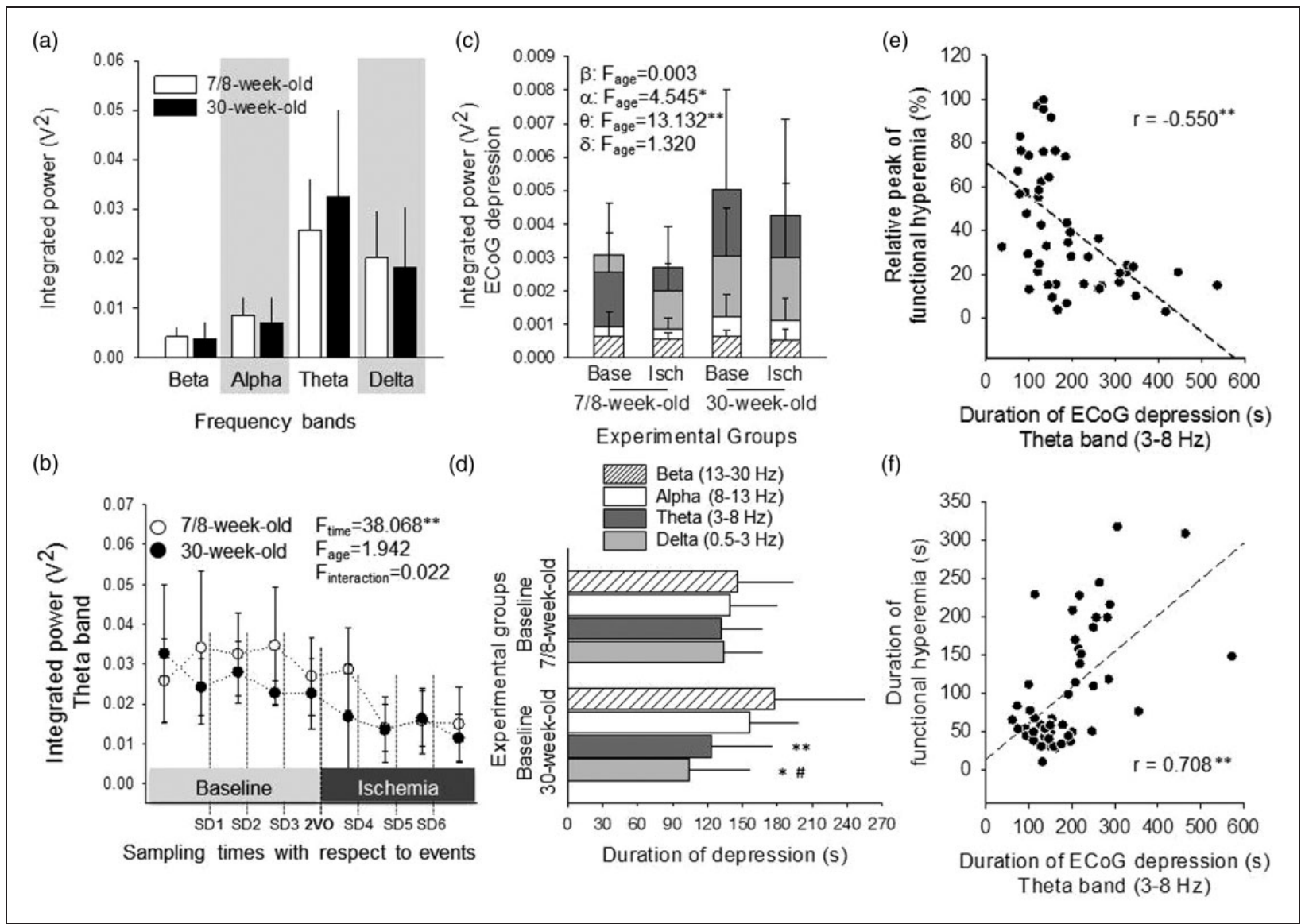

Figure 6. Spectrum analysis of the ECoG for individual frequency bands during SD events. (a) ECoG power of each frequency band, for $60 \mathrm{~s}$ baseline at the very start of the experimental protocol, prior to any intervention. No significant difference between age groups was observed, for any of the frequency bands examined (one-way ANOVA). Note that the weight of low frequency bands in the spectrum is greater than that of higher frequency waves, which mainly reflects an inherent feature of ECoG spectra. Because the ECoG frequency bands represent a power spectrum, their values will vary exponentially, meaning the lower-frequency bands will have exponentially greater values than the higher-frequency bands. (b) Baseline ECoG power of the theta band, assessed repeatedly prior to events, in order to follow the distribution of baseline over the course of the experimental protocol. The first pair of symbols corresponds with theta in Panel A. Repeated measures ANOVA revealed that the ECoG power of baseline decreased, especially during ischemia, irrespective of age. (c) ECoG power of distinct frequency bands, calculated for the period of SD-related ECoG depression. The power of individual frequency bands is represented by bars positioned behind each other. Two-way ANOVA considering age and ischemia as its factors revealed that age significantly elevated the power of the alpha and theta bands selectively, irrespective of ischemia. Level of significance was defined as $p<0.05^{*}$ and $p<0.0 \mathrm{I}^{* *}$. (d) Duration of ECoG depression with SDs evoked under baseline condition. The low frequency bands start to recover significantly sooner than the high frequency bands in the 30 -week-old group (one-way ANOVA followed by a Fisher post hoc test, $p<0.05^{\#}$ vs. alpha, $p<0.05^{*}$ and $p<0.0$ I $^{* *}$ vs. beta). (e) Negative correlation between the duration of the SD-related depression on the theta band of the ECoG, and the relative peak of SDcoupled hyperemia. Pearson two-tailed correlation analysis revealed significant association between these data sets $(p<0.0 \mathrm{I} * *)$. ( $f)$ Positive correlation between the duration of the SD-related depression on the theta band of the ECoG, and the duration of SDcoupled hyperemia. Pearson two-tailed correlation analysis indicated significant association between the data sets $(p<0.0 \mathrm{I**})$.

\section{Life span targeted}

The present study aimed at investigating the electric threshold of SD elicitation and the evolution of the SDcoupled hemodynamic response in 7-30-week-old rats, with or without cerebral ischemia. This life span corresponds to human adolescence and young adulthood. ${ }^{21}$ The interpretation of the age-related aspects of the results with respect to previous reports is challenging: Studies on cerebral ischemia, which tackle the issue of age focus mainly either on the very early phase of life (i.e., birth and maturation) or old age (i.e., aging). The data presented here, therefore, may shed light on significant neurophysiological changes that take place during young adulthood. 


\section{Electric threshold to elicit spreading depolarization}

Our recent studies have demonstrated that the threshold of SD elicitation increases with age: In the intact cortex, $\mathrm{K}^{+}$-induced, recurrent SDs occurred less frequently in 10-month-old rats as compared with their 2-month-old counterparts, ${ }^{18}$ and increasing concentration of $\mathrm{K}^{+}$was required to evoke SDs in 2-year-old as compared with 2-month-old rats. ${ }^{15}$ In addition, spontaneous SDs in the ischemic cortex of 2-year-old rats emerged less likely with respect to 9-month-old animals. ${ }^{16}$ These findings urged us to determine at what exact age the threshold of SD elicitation starts to rise, especially in ischemic tissue.

Our present data clearly demonstrate that the electric threshold of SD elicitation markedly increases with ischemia as well as with the progression of lifetime during early adulthood (Figure 3(a)). Furthermore, the ischemia-related threshold elevation is increasingly more obvious with older age.

The ischemia-related increase of SD threshold may be the result of the metabolic state of the tissue, as indicated by the correspondence between lower CBF taken prior to evoking an SD with a higher electric threshold of SD elicitation (Figure 3(b)). The availability of glucose or the level of tissue $\mathrm{pH}$ may reflect tissue metabolic conditions. As such, high concentration of blood glucose $(23-24 \mathrm{mmol} / \mathrm{l})$ was shown to make the tissue resistant to $\mathrm{SD}$ in rats. ${ }^{22}$ Yet, our data on blood glucose level indicated normoglycemic conditions (blood glucose concentration around $9-11 \mathrm{mmol} / \mathrm{l}$ ) in all age groups and revealed no difference between baseline and late ischemia. Therefore, we suspect that factors other than the availability of glucose must determine the ischemia-related inhibition of SD elicitation as seen here. Indeed, a study relying on live brain slices proved that tissue acidosis (i.e., $\mathrm{pH}$ 6.67-6.97, achieved by the elevation of $\mathrm{pCO}_{2}$ or withdrawal of bicarbonate) inhibited the induction and propagation of $\mathrm{K}^{+}$-induced $\mathrm{SD}{ }^{23}$ Decreasing $\mathrm{pH}$ possibly hampers $\mathrm{SD}$ propagation by inhibiting the activity of NMDA receptors. ${ }^{24}$ Tissue acidosis is widely accepted to prevail in cerebral ischemia; $2 \mathrm{VO}$ as imposed here shifts extracellular $\mathrm{pH}$ in the rat cerebral cortex from $\mathrm{pH} 7.3$ to as low as $\mathrm{pH} 6.8$ (our own measurements in the model used here). Therefore, we suggest that the ischemia-related fall of tissue $\mathrm{pH}$ could be a key factor in raising the threshold of SD elicitation as observed here. Finally, increased $\mathrm{K}^{+}$conductance and the gradual accumulation of extracellular $\mathrm{K}^{+}$that occur during ischemia generally contribute to membrane hyperpolarization and repolarization thereby depressing neuronal excitability. ${ }^{25}$ This ionic imbalance may effectively inhibit SD elicitation as well.

The increased threshold of SD elicitation with advancing age during early adulthood may be linked to structural changes in neuronal networks, which may alter the electrophysiological properties of the nervous tissue. The formation and retraction of dendritic spines that host the post-synaptic element of excitatory synapses dynamically changes with brain maturation ${ }^{26}$ and aging, ${ }^{27}$ is heavily involved in SD propagation, ${ }^{28}$ and is negatively affected by SD. ${ }^{29}$ Therefore, we set out to determine the dendritic spine density in the youngest and the oldest animals admitted to our study to contemplate on any potential link between age-related structural changes in the cortex, and the threshold of SD elicitation. Our results exhibited an increased density of dendritic spines on the apical dendrites of cortical layer 3 pyramidal neurons at 30 weeks of age with respect to 8 weeks of age (Figure 4), proving that the histological organization of the cortex undergoes detectable alterations during the life span investigated in this study. Even though a direct link between such fine structural changes and the excitability of neurons is challenging to establish, we speculate that the threshold of SD elicitation may increase with age during early adulthood because of the histological (and connected biochemical) maturation or consolidation of cortical connections.

\section{CBF compensation following ischemia induction and $C B F$ response to $S D$}

Vascular occlusion is immediately followed by a sharp drop of perfusion pressure below the autoregulatory range and a marked reduction of $\mathrm{CBF}$ causing ischemia. In turn, the reduced level of ATP opens $\mathrm{K}_{\mathrm{ATP}}$ channels on vascular smooth muscle cells, causing hyperpolarization and thus vasodilation. In addition, hypoxia rapidly increases adenosine production locally, also promoting vasodilation. ${ }^{30}$ This, together with compensating collateral flow or vascular remodeling contributes to a partial recovery of perfusion. ${ }^{31}$ Our previous work focusing on aging demonstrated that the ischemiarelated perfusion deficit progressively deepened - rather than partially recovered - in the brain of 2-year-old animals as opposed to young adults in global and focal ischemia models. ${ }^{15,16}$ Here, we show that the impairment in flow compensation becomes first detectable as early as 30 weeks of age in the Sprague-Dawley rat (Figure 2). Taken the young adult age screened here, declining CBF compensation is suspected to root in altered vasoreactivity, rather than an age-related rarefaction of collateral vascular networks. ${ }^{32}$ Possibly, altered characteristics of $\mathrm{K}_{\mathrm{ATP}}$ channel opening, ineffective adenosine signaling or restricted NO availability could potentially account for the less efficient flow compensation shortly after ischemia onset in our oldest age group.

The present results on the kinetics of the SD-related CBF response confirmed that the amplitude of 
hyperemia decreased, while the duration of hyperemia increased under ischemia in the young adult brain. ${ }^{15,33}$ Nevertheless, inverse neurovascular coupling, as encountered frequently in the aged ischemic brain earlier, ${ }^{15,16}$ did not evolve. Instead, an augmented, ischemia-related insufficiency of the SD-related CBF response was implied in the 30 -week-old animals by the lowest absolute peak of the SD-associated hyperemia under ischemia (Figure 5(b)). Clearly, the failing flow compensation to ischemia set the scene for the reduced absolute amplitude of hyperemia in the 30week-old group. At the same time, the relative amplitude of hyperemia appeared similar across age groups. Taken together, we conclude that neurovascular coupling during SD was impaired by ischemia, but this impairment was not enhanced by increasing age in young adult rats.

\section{Distinct features of the ECoG power spectrum}

Our data demonstrate that the alpha and theta frequency bands are less depressed during SD in the 30week-old group as compared with the 7/8-week-old group (Figure 6(c)). ECoG was acquired through a glass capillary electrode inserted into the cortex, also used for the recording of the DC potential. The ECoG signal, therefore, represented a local field potential that contains both action potentials and other membrane potential-derived fluctuations in a small neuronal volume, approximately within a few hundred micrometers from the electrode tip. ${ }^{34}$ A very important factor determining the ECoG signal as recorded here is the cellular-synaptic architectural organization of the neuronal network. ${ }^{34}$ Here, we show that the dendritic spine density, for example, is age-dependent in young adulthood. It is thus conceivable that the age-related alterations of dendritic geometry may impact on selective frequencies of the ECoG power during the SDrelated depression, which might be represented in the less obvious depression of given frequency bands (Figure 6(c)).

The duration of SD-related depression appeared shorter specifically on the delta and theta bands in the 30 -week-old group, as compared with the $7 / 8$-week-old group (Figure 6(d)). This suggests that the electrical activity in the low frequency range resumed sooner after the passage of SD at older age. These data are in full agreement with our previous report showing that the SD-related silence of the full ECoG is shortened in 10-month-old as compared with 2-month-old rats. ${ }^{18}$ Earlier, we speculated that the shorter ECoG depression with SD in the older animals could indicate the involvement of fewer neurons at any given time during SD propagation, thereby creating a narrower wave of depression. Taken that the low frequency component of the ECoG is thought to arrive at the tip of the recording electrode from more distant sites, ${ }^{34}$ the current data showing the shorter depression of the low frequency ECoG components support the concept of a narrower SD-related depression wave with older age.

The duration of $\mathrm{ECoG}$ depression appears to be associated with the features of the SD-coupled CBF response. The present correlation analyses demonstrate that a larger peak of SD-related hyperemia coincides with shorter depression of the ECoG (Figure 6(e)). This is complementary to previous reports describing that deeper initial hypoperfusion of the CBF response coincides with longer ECoG depression, ${ }^{18}$ and that longer duration of the initial hypoperfusion corresponds with longer DC shift duration with $\mathrm{SD}$, in experimental models, as well as in patients of aneurysmal subarachnoid haemorrhage. ${ }^{35,36}$ Taken together, a more prominent dilator component in the $\mathrm{CBF}$ response (i.e., smaller magnitude of initial hypoperfusion or larger amplitude of hyperemia) appears to facilitate the faster return of the cortex' electrical activity after the passage of SD.

Longer ECoG depression with SD was also associated with longer hyperemia (Figure 6(f)). Likewise, longer duration of the DC shift indicative of SD coincided with longer hyperemia. ${ }^{15}$ Since the recovery of resting membrane potential and thus normal electrical activity of the nervous tissue require the activity of the ATP-consuming $\mathrm{Na}^{+}$pump, ${ }^{4}$ the return of CBF to baseline after hyperemia may be postponed by the continuing energy need, reflected by the longer duration of the electric silence with SD.

\section{Conclusions}

Recurrent SDs occur in ischemic and traumatic brain injury at high incidence and are thought to usher the expansion of tissue damage. ${ }^{1,2,4,37,38}$ The data presented here reveal that the susceptibility of the nervous tissue to SD is highest at adolescent or young adult age; yet, the prospect of successful recovery from ischemic or traumatic brain injury is considerably better for young as compared with old patients. ${ }^{39,40}$ These pieces of information would be apparently conflicting if all SDs possessed equally injurious potential.

Researchers now agree that SDs coupled with spreading ischemia promote lesion progression. ${ }^{4}$ Yet, individual SDs with hyperemic CBF response have not been explicitly proven either harmless or injurious in tissue at risk, especially because the SD-coupled CBF response, which appears hyperemic at the site of detection might turn into spreading ischemia as it propagates in areas not screened. Further, even though the longer cumulative duration of recurrent SDs was associated 
with the expansion of tissue damage in focal ischemia, ${ }^{11}$ this does not directly infer that the longer cumulative duration is the outcome of a higher number of eventsindeed, the duration of individual SD events can vary considerably. ${ }^{4}$ Finally, we have previously demonstrated that although only a few SDs occur spontaneously in the aged ischemic brain, these events cause more damage than a higher number of SDs in the young brain, as indicated by a remarkable delay of repolarization after SD and the associated evolution of perfusion deficit. ${ }^{16}$

Taken together, the low threshold of SD elicitation indicates the brain's high susceptibility for SD generation at young age, but it may not directly put the tissue at higher risk for SD-related injury. This notion needs to be explored further in subsequent experimental and clinical studies.

\section{Funding}

The author(s) disclosed receipt of the following financial support for the research, authorship, and/or publication of this article: This work was supported by grants from the Hungarian Scientific Research Fund (Grant No. K111923); the Hungarian Brain Research Program (Grant No. KTIA_13_NAP-A-I/13); TÁMOP-4.2.2.D-15/1/KONV2015-0024; and the Bolyai János Research Scholarship of the Hungarian Academy of Sciences (BO/00327/14/5, to EF).

\section{Declaration of conflicting interests}

The author(s) declared no potential conflicts of interest with respect to the research, authorship, and/or publication of this article.

\section{Authors' contributions}

Contributions are defined as listed among the "Editorial Policies" document of JCBFM^-Péter Hertelendy: acquisition, analysis and interpretation of data, drafting the article; Ákos Menyhárt: acquisition and interpretation of data, drafting the article; Péter Makra: analysis of data, drafting the article; Zoltán Süle: acquisition and analysis of data, drafting the article; Tamás Kiss: acquisition and analysis of data; Gergely Tóth: acquisition of data; Orsolya Ivánkovits-Kiss: acquisition and analysis of data, drafting the article; Ferenc Bari: revising the manuscript critically for important intellectual content, final approval of the version to be published; Eszter Farkas: substantial contributions to conception and design, drafting the article and revising it critically for important intellectual content, final approval of the version to be published.

\section{References}

1. Dreier JP, Woitzik J, Fabricius M, et al. Delayed ischaemic neurological deficits after subarachnoid haemorrhage are associated with clusters of spreading depolarizations. Brain 2006; 129: 3224-3237.
2. Hartings JA, Watanabe T, Bullock MR, et al. Spreading depolarizations have prolonged direct current shifts and are associated with poor outcome in brain trauma. Brain 2011; 134: 1529-1540.

3. Woitzik J, Hecht N, Pinczolits A, et al. Propagation of cortical spreading depolarization in the human cortex after malignant stroke. Neurology 2013; 80: 1095-1102.

4. Dreier JP. The role of spreading depression, spreading depolarization and spreading ischemia in neurological disease. Nat Med 2011; 17: 439-447.

5. Somjen GG. Mechanisms of spreading depression and hypoxic spreading depression-like depolarization. Physiol Rev 2001; 81: 1065-1096.

6. Ayata $\mathrm{C}$ and Lauritzen M. Spreading depression, spreading depolarizations, and the cerebral vasculature. Physiol Rev 2015; 95: 953-993.

7. Dreier JP, Körner K, Ebert N, et al. Nitric oxide scavenging by hemoglobin or nitric oxide synthase inhibition by $\mathrm{N}$-nitro-L-arginine induces cortical spreading ischemia when $\mathrm{K}+$ is increased in the subarachnoid space. J Cereb Blood Flow Metab 1998; 18: 978-990.

8. Chen RL, Balami JS, Esiri MM, et al. Ischemic stroke in the elderly: an overview of evidence. Nat Rev Neurol 2010; 6: 256-265.

9. Ingall T, Asplund K, Mähönen M, et al. A multinational comparison of subarachnoid hemorrhage epidemiology in the WHO MONICA stroke study. Stroke 2000; 31: 1054-1061.

10. Bruns $\mathrm{J} \mathrm{Jr}$ and Hauser WA. The epidemiology of traumatic brain injury: a review. Epilepsia 2003; 44: 2-10.

11. Dijkhuizen RM, Beekwilder JP, van der Worp HB, et al. Correlation between tissue depolarizations and damage in focal ischemic rat brain. Brain Res 1999; 840: 194-205.

12. Nedergaard M. Spreading depression as a contributor to ischemic brain damage. Adv Neurol 1996; 71: 75-84.

13. Richter F, Lehmenkühler A, Fechner R, et al. Postnatal conditioning for spreading cortical depression in the rat brain. Brain Res Dev Brain Res 1998; 106: 217-221.

14. Maslarova A, Alam M, Reiffurth C, et al. Chronically epileptic human and rat neocortex display a similar resistance against spreading depolarization in vitro. Stroke 2011; 42: 2917-2922.

15. MenyhártÁ, Makra P, Szepes BÉ, et al. High incidence of adverse cerebral blood flow responses to spreading depolarization in the aged ischemic rat brain. Neurobiol Aging 2015; 36: 3269-3277.

16. Clark D, Institoris A, Kozák G, et al. Impact of aging on spreading depolarizations induced by focal brain ischemia in rats. Neurobiol Aging 2014; 35: 2803-2811.

17. Leão AAP. Spreading depression of activity in the cerebral cortex. J Neurophysiol 1944; 7: 359-390.

18. Farkas E, Obrenovitch TP, InstitórisÁ, et al. Effects of early aging and cerebral hypoperfusion on spreading depression in rats. Neurobiol Aging 2011; 32: 1707-1715.

19. Glaser EM and Van der Loos H. Analysis of thick brain sections by obverse-reverse computer microscopy: application of a new, high clarity Golgi-Nissl stain. J Neurosci Methods 1981; 4: 117-125.

20. von Bornstädt D, Houben T, Seidel JL, et al. Supplydemand mismatch transients in susceptible peri-infarct 
hot zones explain the origins of spreading injury depolarizations. Neuron 2015; 85: 1117-1131.

21. Sengupta P. The laboratory rat: relating its age with human's. Int J Prev Med 2013; 4: 624-630.

22. Hoffmann U, Sukhotinsky I, Eikermann-Haerter K, et al. Glucose modulation of spreading depression susceptibility. J Cereb Blood Flow Metab 2013; 33: 191-195.

23. Tong CK and Chesler M. Modulation of spreading depression by changes in extracellular $\mathrm{pH}$. J Neurophysiol 2000; 84: 2449-2457.

24. Tang CM, Dichter M and Morad M. Modulation of the N-methyl-D-aspartate channel by extracellular H+. Proc Natl Acad Sci USA 1990; 87: 6445-6449.

25. Shah NH and Aizenman E. Voltage-gated potassium channels at the crossroads of neuronal function, ischemic tolerance, and neurodegeneration. Transl Stroke Res 2014; 5: 38-58.

26. Harris KM, Jensen FE and Tsao B. Three-dimensional structure of dendritic spines and synapses in rat hippocampus (CA1) at postnatal day 15 and adult ages: implications for the maturation of synaptic physiology and long-term potentiation. J Neurosci 1992; 12: 2685-2705.

27. Dickstein DL, Weaver CM, Luebke JI, et al. Dendritic spine changes associated with normal aging. Neuroscience 2013; 251: 21-32.

28. Herreras O and Somjen GG. Propagation of spreading depression among dendrites and somata of the same cell population. Brain Res 1993; 610: 276-282.

29. Risher WC, Ard D, Yuan J, et al. Recurrent spontaneous spreading depolarizations facilitate acute dendritic injury in the ischemic penumbra. $J$ Neurosci 2010; 30: 9859-9868.

30. Cippola MJ. The cerebral circulation. Morgan \& Claypool Life Sciences: San Rafael, CA, 2009.

31. Lapi D and Colantuoni A. Remodeling of cerebral microcirculation after ischemia-reperfusion. J Vasc Res 2015; 52: $22-31$.
32. Faber JE, Zhang H, Lassance-Soares RM, et al. Aging causes collateral rarefaction and increased severity of ischemic injury in multiple tissues. Arterioscler Thromb Vasc Biol 2011; 31: 1748-1756.

33. Feuerstein D, Takagaki M, Gramer M, et al. Detecting tissue deterioration after brain injury: regional blood flow level versus capacity to raise blood flow. J Cereb Blood Flow Metab 2014; 34: 1117-1127.

34. Buzsáki G, Anastassiou CA and Koch C. The origin of extracellular fields and currents-EEG, ECoG, LFP and spikes. Nat Rev Neurosci 2012; 13: 407-420.

35. Sukhotinsky I, Dilekoz E, Moskowitz MA, et al. Hypoxia and hypotension transform the blood flow response to cortical spreading depression from hyperemia into hypoperfusion in the rat. J Cereb Blood Flow Metab 2008; 28: 1369-1376.

36. Dreier JP, Major S, Manning A, et al. Cortical spreading ischaemia is a novel process involved in ischaemic damage in patients with aneurysmal subarachnoid haemorrhage. Brain 2009; 132: 1866-1881.

37. Hartings JA, Strong AJ, Fabricius M, et al. Spreading depolarizations and late secondary insults after traumatic brain injury. J Neurotrauma 2009; 26: 1857-1866.

38. Hinzman JM, Andaluz N, Shutter LA, et al. Inverse neurovascular coupling to cortical spreading depolarizations in severe brain trauma. Brain 2014; 137: 2960-2972.

39. Hukkelhoven CW, Steyerberg EW, Rampen AJ, et al. Patient age and outcome following severe traumatic brain injury: an analysis of 5600 patients. J Neurosurg 2003; 99: 666-673.

40. Lanzino G, Kassell NF, Germanson TP, et al. Age and outcome after aneurysmal subarachnoid hemorrhage: why do older patients fare worse? J Neurosurg 1996; 85: 410-418. 
III. Varga, D.P., Puskás, T., Menyhárt, Á., Hertelendy, P., Zölei-Szénási, D., Tóth, R., Ivánkovits-Kiss, O., Bari, F., Farkas, E.

Contribution of prostanoid signaling to the evolution of spreading depolarization and the associated cerebral blood flow response.

Sci Rep. 2016 Aug 10;6:31402. doi: 10.1038/srep31402.

IF : $\mathbf{5 . 5 2 5}$ 


\section{SCIENTIFIC REP}

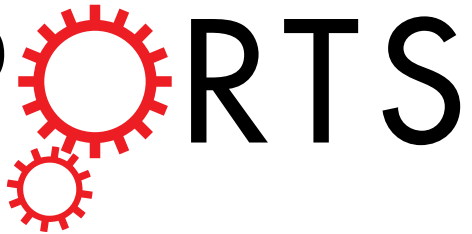

OPEN

Contribution of prostanoid signaling to the evolution of spreading depolarization and the

Received: 13 May 2016

Accepted: 19 July 2016

Published: 10 August 2016 associated cerebral blood flow response

\author{
Dániel Péter Varga*, Tamás Puskás*, Ákos Menyhárt, Péter Hertelendy, Dániel Zölei-Szénási, \\ Réka Tóth, Orsolya Ivánkovits-Kiss, Ferenc Bari \& Eszter Farkas
}

The significance of prostanoid signaling in neurovascular coupling during somatosensory stimulation is increasingly more appreciated, yet its involvement in mediating the cerebral blood flow (CBF) response to spreading depolarization (SD) has remained inconclusive. Selective cyclooxygenase (COX) enzyme inhibitors (NS-398, SC-560) or an antagonist (L161,982) of the EP4 type prostaglandin E2 receptor were applied topically to a cranial window over the parietal cortex of isoflurane-anesthetized SpragueDawley rats $(n=60)$. Global forebrain ischemia was induced by occlusion of both common carotid arteries in half of the animals. SDs were triggered by the topical application of $1 \mathrm{M} \mathrm{KCl}$. SD occurrence was confirmed by the acquisition of DC potential, and CBF variations were recorded by laser-Doppler flowmetry. EP4 receptor antagonism significantly decreased peak hyperemia and augmented post-SD oligemia in the intact but not in the ischemic cortex. COX-1 inhibition and EP4 receptor blockade markedly delayed repolarization after SD in the ischemic but not in the intact brain. COX-2 inhibition achieved no significant effect on any of the end points taken. The data suggest, that activation of EP4 receptors initiates vasodilation in response to SD in the intact brain, and - together with COX-1 derived prostanoids - shortens SD duration in the acute phase of ischemia.

Recurrent spreading depolarizations (SDs) are slowly propagating waves of electrical silence in the cerebral gray matter $^{1}$ that occur spontaneously in the injured brain ${ }^{2,3}$. Recurrent SD events have recently been recognized to exacerbate ischemic brain injury in patients of subarachnoid hemorrhage, stroke or traumatic brain injury ${ }^{4}$, and are being promoted as a causal biomarker assessed in neurocritical care to indicate the degree of metabolic failure in the brain tissue ${ }^{5}$.

SDs are coupled with typical changes in local cerebral blood flow $(\mathrm{CBF})^{6}$. In the rat - and most probably in human - the physiological pattern of the SD-associated CBF response includes four sequential components: (i) an initial, brief hypoperfusion; (ii) a marked, transient peak hyperemia; (iii) a less obvious late hyperemia; and (iv) a sustained hypoperfusion also known as spreading oligemia or post-SD oligemia ${ }^{6}$. The duration and magnitude of these four elements in the CBF response is variable, with the peak hyperemic component being the most conspicuous. In the ischemic brain, the $\mathrm{CBF}$ response to $\mathrm{SD}$ is more dominated by vasoconstrictive elements, leading to diminishing hyperemia and more prevalent hypoemia ${ }^{7-10}$. In the most severe form, the hypoemic element completely outweighs hyperemia, and turns into spreading ischemia ${ }^{11}$. This atypical SD-associated CBF variation in the injured brain aggravates metabolic supply-demand mismatch in the tissue, and can delay recovery from SD thereby increasing the risk of irreversible depolarization and neuronal cell death.

The regulation of the SD-related CBF response appears to be rather complex, and the discrimination of any specific individual mediator poses a considerable challenge ${ }^{6}$. In physiological neurovascular coupling during somatosensory stimulation, prostanoids have emerged as potent vasoactive metabolites ${ }^{12,13}$. A major pathway

Department of Medical Physics and Informatics, Faculty of Medicine \& Faculty of Science and Informatics, University of Szeged, H-6720 Szeged, Korányi fasor 9, Hungary. *These authors contributed equally to this work. Correspondence and requests for materials should be addressed to E.F. (email: farkas.eszter.1@med.u-szeged.hu) 
$\mathrm{A}$

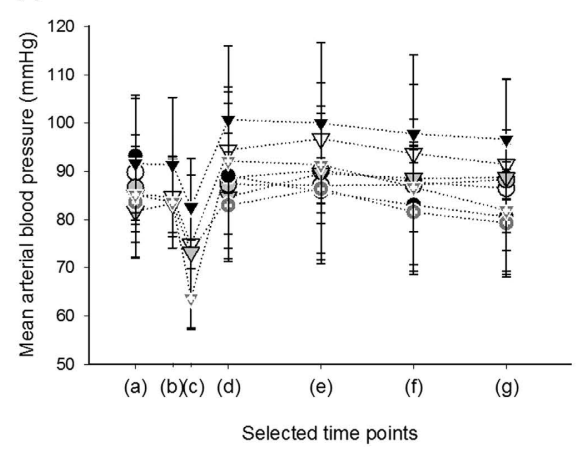

B

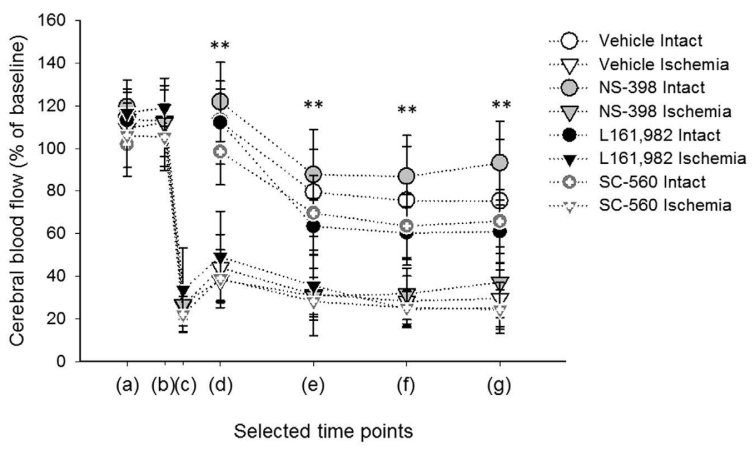

Figure 1. Mean arterial blood pressure (MABP; in (A) and drift of local cerebral blood flow (CBF; in (B) over the experimental protocol. Selected time points for sampling have been defined as follows: (a) at $35 \mathrm{~min}$ after the initiation of pharmacological treatments; (b) immediately before ischemia induction; (c) MABP (A) or CBF (B) minimum after ischemia induction; (d) prior to SD1; (e) prior to SD2; (f) prior to SD3; (g) prior to SD4. Data are given as mean \pm stdev. Statistical analysis relied on a repeated measures paradigm followed by a Fisher post hoc test. Ischemia - but not treatment - had a significant effect on the baseline drift of CBF. Level of significance was determined as $* * \mathrm{p}<0.01$ vs. respective Intact group.

leading to vasodilator prostanoid synthesis involves cyclooxygenase-2 (COX-2), a rate limiting, inducible enzyme using arachidonic acid as its substrate. COX-2 is expressed in cortical pyramidal neurons ${ }^{14}$, and is located in perivascular nerve terminals along intraparenchymal penetrating arterioles and capillaries ${ }^{15}$. Most importantly, COX-2 products have emerged as mediators of functional hyperemia to somatosensory stimulation ${ }^{13,16}$. A COX-2 derived vasoactive mediator produced by the downstream enzyme prostaglandin E synthase is prostaglandin E2 $\left(\mathrm{PGE}_{2}\right)^{17}$, which causes vasodilation by binding to its receptors (EP2 and EP4 receptors) located in the vascular wall in the brain ${ }^{13,14,18}$. In contrast with the COX-2 route, the role of the constitutive COX-1 enzyme (which, in the context of physiological neurovascular coupling, is argued to be expressed in astrocytes $)^{19}$ in shaping the CBF response to neuronal activity has remained controversial ${ }^{19}$. Selective COX-1 inhibition blocked the evolution of hyperemia in response to odorant stimulation ${ }^{20}$, or uncaging of $\mathrm{Ca}^{2+}$ in perivascular astrocytic endfeet ${ }^{21}$, yet it exerted no impact on the CBF response to whisker stimulation ${ }^{22-24}$.

Arachidonc acid metabolites could possibly play a central role in mediating the CBF response to SD because spreading depolarization coincides with a considerable accumulation of arachidonic acid in the cortex ${ }^{25}$, and a significant elevation of prostanoid concentration (e.g. $\mathrm{PGE}_{2}$ ) in the cerebrospinal fluid ${ }^{26}$. Yet, in contrast with the dominant vasodilator effect of prostaglandins in response to somatonsensory stimulation ${ }^{13}$, arachidonic acid metabolites released due to SD were found to be vasoconstrictive: First, the non-selective inhibition of COX enzymes (i.e. application of indomethacin) caused pial vasodilation with $\mathrm{SD}$, and diminished vasoconstriction underlying the post-SD oligemia ${ }^{26}$, Second, synthesis of the vasoconstrictive hydroxyeicosatetraeonic acid (20-HETE) by the P450 epoxygenase enzyme located in vascular smooth muscle cells was demonstrated in response to SD, and the pharmacological blockade of its synthesis ameliorated the post SD oligemia ${ }^{27}$. Nonetheless, the selective effect of COX-1 or COX-2 products on the SD-associated CBF response has not been revealed, although the potential involvement of COX-2 is conceivable, because COX-2 mRNA and protein were found upregulated in cortical neurons in association with $\mathrm{SD}^{28}$.

In summary, even though the significance of the COX-2 pathway in neurovascular coupling during somatosensory stimulation is getting increasingly more appreciated ${ }^{12,13}$, its involvement in mediating the CBF response to SD has remained inconclusive and is far from being understood ${ }^{6}$. In addition, COX-2 is remarkably upregulated in the ischemic brain during the period over which deleterious SDs occur spontaneously in rodent ischemia model ${ }^{29}$, but there is essentially no evidence as to whether the ischemia-related COX-2 elevation modulates the $\mathrm{CBF}$ response to $\mathrm{SD}$.

Taken together, here we set out to determine systematically whether COX-2 or COX-1 derived metabolites specifically the vasodilator $\mathrm{PGE}_{2}{ }^{18,30}$ - contribute to the evolution of the SD-coupled hyperemia in the intact or the ischemic rat cerebral cortex. We also sought to assess any potential involvement of prostanoid signaling in the evolution of SD itself.

\section{Results}

General physiological variables. Topical application of drugs was chosen in order to avoid potential systemic side effects. Measured values of mean arterial blood pressure (MABP) and the outcome of blood gas analysis confirmed no difference in the systemic variables assessed in various treatment groups. Specifically, Fig. 1A demonstrates that MABP did not change significantly over the experimental protocol, and was similar across experimental groups. Arterial blood gas analysis showed that all investigated variables were within the physiological range at the start of the experiments (i.e. $\mathrm{pO}_{2}: 133 \pm 28 \mathrm{mmHg}_{\mathrm{mCO}}: 32 \pm 6 \mathrm{mmHg}, \mathrm{pH}$ : $7.39 \pm 0.02, \mathrm{HCO}_{3}: 19.19 \pm 4.54 \mathrm{mmol} / \mathrm{l}$, glucose: $\left.5.21 \pm 1.71 \mathrm{mmol} / \mathrm{l}\right)$ and prior to the termination of the experiments (i.e. $\mathrm{pO}_{2}: 125 \pm 29 \mathrm{mmHg}, \mathrm{pCO}_{2}: 38 \pm 11 \mathrm{mmHg}, \mathrm{pH}: 7.37 \pm 0.08, \mathrm{HCO}_{3}: 21.49 \pm 3.44 \mathrm{mmol} / \mathrm{l}$, glucose: $6.11 \pm 1.92 \mathrm{mmol} / \mathrm{l}$ ). Baseline CBF was obviously reduced significantly due to ischemia induction (from $112 \pm 15$ to $27 \pm 13 \%$ as an average), but the various treatments did not exert any significant impact (Fig. 1B). 
A

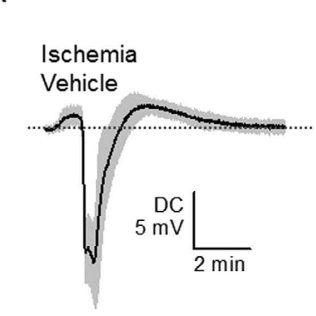

B

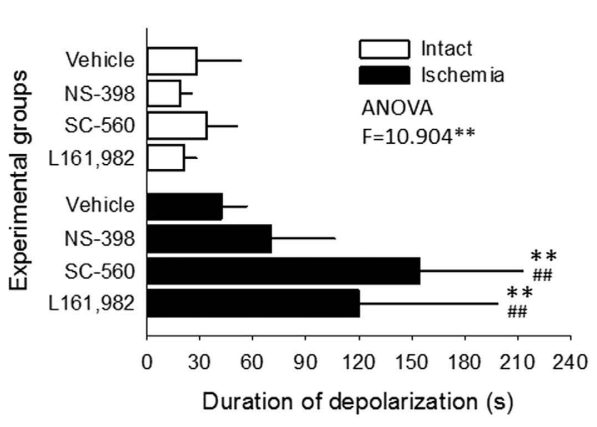

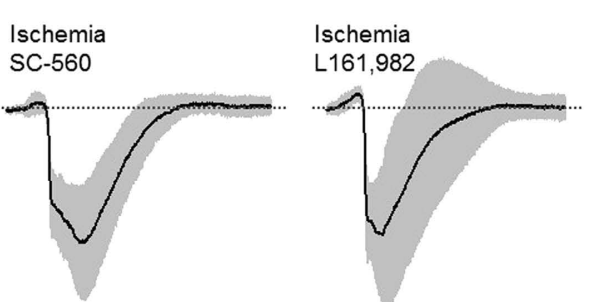

C

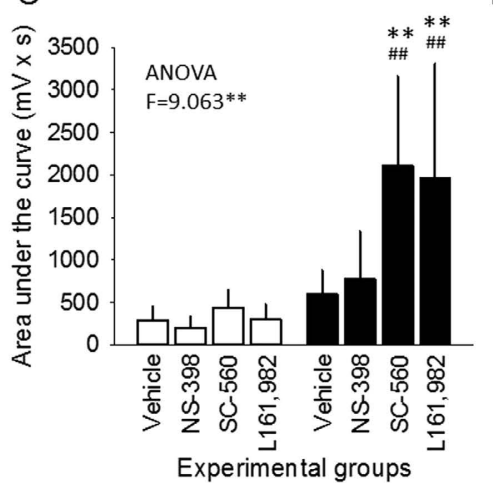

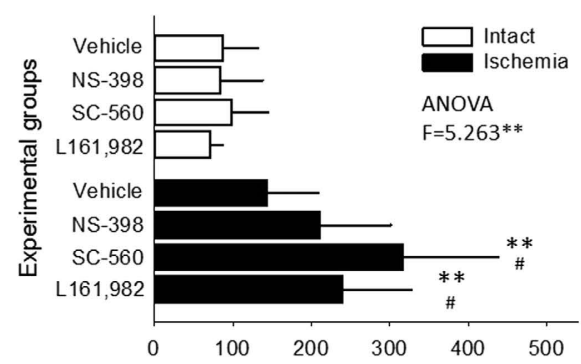

E

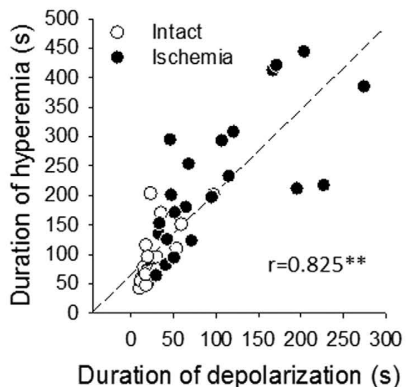

Duration of depolarization (s)

Figure 2. The impact of pharmacological treatments on the kinetics of the spreading depolarization (SD)-related, transient negative shift of the direct current (DC) potential. All panels demonstrate data for recurrent SDs. (A) DC potential traces are mean \pm stdev, standing for Vehicle, SC-560, and L161,982 treatment under ischemia. (B) Duration of the negative shift of the DC potential taken at half amplitude. (C) Area under the curve of the negative shift of the DC potential. Note the selective impact of COX-1 inhibition (SC-560) and EP4 receptor blockade (L161,982) under ischemia. (D) Duration of the cerebral blood flow (CBF) response to SD taken at half amplitude of peak hyperemia. (E) Correlation between the duration of the SD-related negative DC shift and the associated CBF response; all groups are shown. Data shown in Panels B-D are given as mean \pm stdev. Statistical analysis relied on a one-way analysis of variance (ANOVA) model followed by a Fisher post hoc test. Statistical significance was determined as ${ }^{*} \mathrm{p}<0.01$ vs. respective Ischemia, and ${ }^{\#} \mathrm{p}<0.05$ or ${ }^{\# \#} \mathrm{p}<0.01$ vs. respective Vehicle. The correlation presented in Panel E was tested with a Pearson one-tailed correlation analysis $(* * \mathrm{p}<0.01)$.

Direct current potential signature of spreading depolarization. Pharmacological manipulation proved to be ineffective on the kinetics of SD in the Intact groups. Conversely, the DC potential signature of SD was markedly and selectively altered by SC-560 and L161,982 treatment in the Ischemic group (Fig. 2A). As such, the duration of the negative DC shift at half amplitude was substantially increased for both the first SD (117 \pm 32 and $95 \pm 64$, vs. $43 \pm 15 \mathrm{~s}$; Ischemic SC-560 and Ischemic L161,982 vs. Ischemic Vehicle), and recurrent SDs ( $154 \pm 58$ and $120 \pm 79$, vs. $42 \pm 14$ s; Ischemic SC-560 and Ischemic L161,982 vs. Ischemic Vehicle) (Fig. 2B). Similarly, the magnitude of the DC shift characterized by the area under the curve greatly increased due to SC-560 and L161,982 treatment in combination with ischemia, for both the first SD (1499 \pm 340 and $1478 \pm 1263$, vs. $574 \pm 384 \mathrm{mV} \mathrm{x} \mathrm{s;} \mathrm{Ischemic} \mathrm{SC-560} \mathrm{and} \mathrm{Ischemic} \mathrm{L161,982} \mathrm{vs.} \mathrm{Ischemic} \mathrm{Vehicle),} \mathrm{and} \mathrm{recurrent} \mathrm{SDs} \mathrm{(2104} \pm 1056$ and $1958 \pm 1357$, vs. $598 \pm 281 \mathrm{mV}$ x s; Ischemic SC-560 and Ischemic L161,982 vs. Ischemic Vehicle) (Fig. 2C). In addition, SC-560 and L161,982 treatment markedly reduced the amplitude of post-SD hyperpolarization (e.g. recurrent SDs: $1.16 \pm 0.50$ and $1.40 \pm 0.62$, vs. $2.65 \pm 0.39 \mathrm{mV}$; Ischemic SC-560 and Ischemic L161,982 vs. Ischemic Vehicle), which also concerned only the ischemic animals but not the intact ones. In contrast, NS-398 treatment proved to be statistically ineffective for all the above variables as compared with Vehicle.

Cerebral blood flow response to spreading depolarization. In contrast with the analysis of the DC potential signature of SD, the impact of pharmacological treatments on the CBF response could be discriminated in the intact - but not in the ischemic - animals. The selective COX enzyme inhibitors NS-398 and SC-560 did not exert any clear influence on the evolution of the SD-related CBF response, as all the examined variables remained unaltered by the treatments.

On the other hand, the EP4 receptor blocker L161,982 selectively reduced the relative amplitude of peak hyperemia with the first SD ( $21 \pm 11$ vs. $51 \pm 38 \%$, Intact L161,982 vs. Intact Vehicle), and recurrent SDs ( $50 \pm 21$ vs. $76 \pm 37 \%$, Intact L161,982 vs. Intact Vehicle). In fact, L161,982 lowered the amplitude of the hyperemic element of the CBF response to near the level observed for the ischemic group (first SD: $21 \pm 11$ vs. $15 \pm 8 \%$, L161,982 Intact vs. L161,982 Ischemic), as indicated by the loss of statistically significant difference otherwise obvious between the intact-ischemic group pairs (i.e. Vehicle, NS-398 or SC-560 treated) (Fig. 3B). Further, L161,982 augmented the relative amplitude of post-SD oligemia with the first SD (58 \pm 13 vs. $40 \pm 14 \%$, Intact L161,982 vs. Intact Vehicle) (Fig. 3A,C). Based on these results, the selective EP4 receptor blocker L161,982 appeared to achieve a general loss of a dilatory element prevalent throughout the CBF response, as reflected by a reduction 

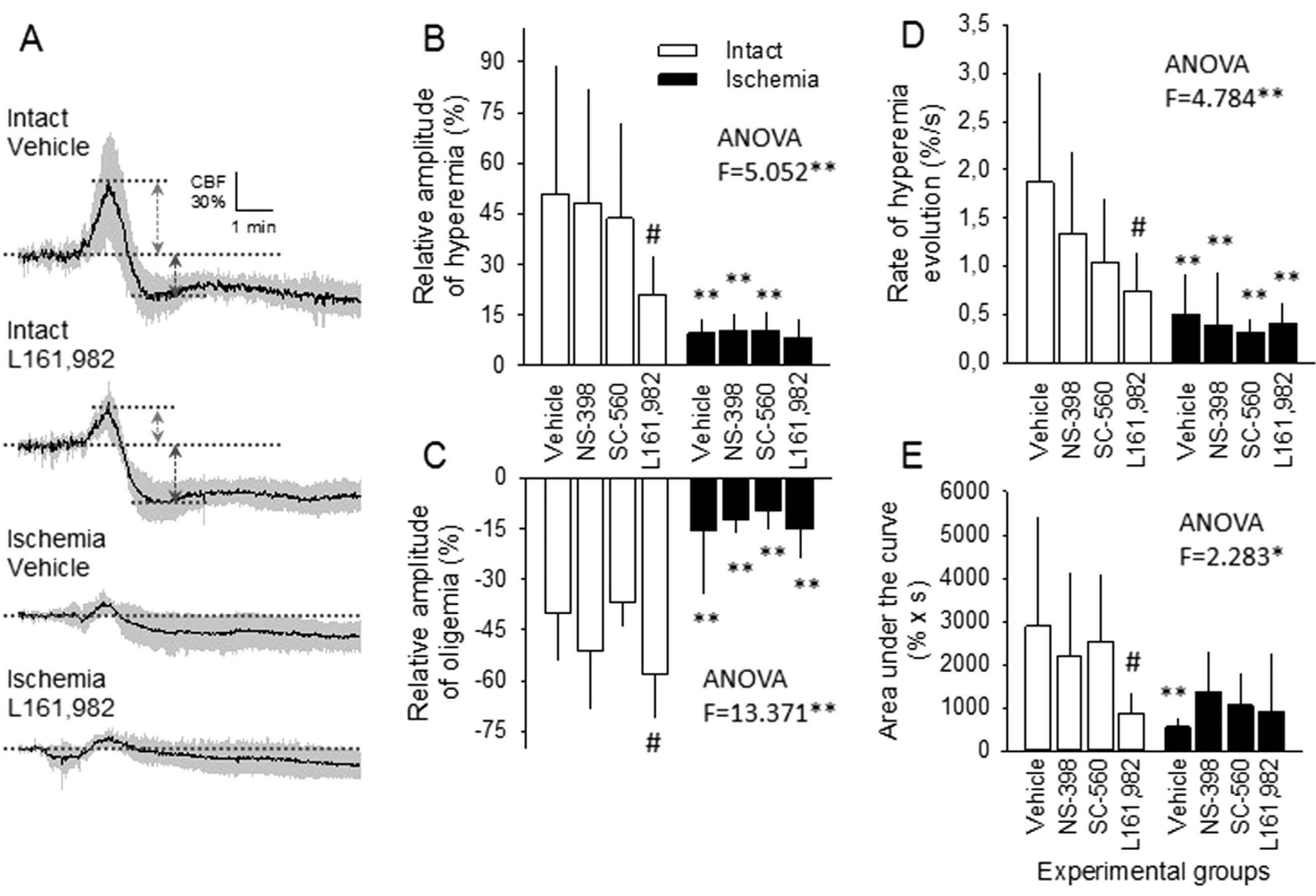

Figure 3. The impact of pharmacological treatments on the kinetics of the spreading depolarization (SD)related cerebral blood flow (CBF) response. All panels demonstrate data for the first SD. A, CBF traces are mean \pm stdev for each group presented. (B) Maximum amplitude of peak hyperemia; (C) Minimum amplitude of post-SD oligemia; (D), Rate of hyperemia evolution associated with SD; (E), Magnitude of hyperemia expressed as area under the curve. Data in Panels B-E are given as mean \pm stdev. Statistical analysis relied on a one-way analysis of variance (ANOVA) model followed by a Fisher post hoc test. Statistical significance was determined as $* \mathrm{p}<0.05$ and $* * \mathrm{p}<0.01$ vs. respective Intact, and ${ }^{*} \mathrm{p}<0.05$ vs. respective Vehicle. Note the selective effect of L161,982 (i.e. EP4 receptor antagonism) under Intact condition.

of peak hyperemia with a degree matching the deepening of the post-SD oligemia (Fig. 3A). This observation was confirmed by calculating the flow difference between peak hyperemia and the minimum point of post-SD oligemia, providing the exploited range of vasoregulation during the $\mathrm{CBF}$ response, which was unchanged due to treatments in the intact groups ( $87 \pm 32 \mathrm{pp}, \%$ in average).

To further prove the selective effect of L161,982 on the SD-related CBF response, hyperemia evolution significantly decelerated due to L161,982 treatment as demonstrated by its shallower upward slope with the first SD $(0.75 \pm 0.38$ vs. $1.88 \pm 1.13 \% / s$, Intact L161,982 vs. Intact Vehicle) (Fig. 3D), and recurrent SDs (1.05 \pm 0.43 vs. $1.60 \pm 0.70 \% / \mathrm{s}$, Intact L161,982 vs. Intact Vehicle). Finally, the magnitude of hyperemia expressed as the area under the curve was also significantly decreased by L161,982 for the first SD ( $874 \pm 462$ vs. $2885 \pm 2543 \%$ x s, Intact L161,982 vs. Intact Vehicle) (Fig. 3E), and recurrent SDs (5532 \pm 3643 vs. $7448 \pm 2072 \%$ x s, Intact L161,982 vs. Intact Vehicle).

The duration of the hyperemic element of the $\mathrm{CBF}$ response appeared to deserve separate consideration, as the changes due to pharmacological treatments seemed to be consistent with the results derived from the DC potential signature of SD (Fig. 2), rather than other variables assessed for the CBF response (Fig. 3). Indeed, the duration of hyperemia markedly elongated in the ischemic animals, especially due to treatment with SC-560 and L161,982 (e.g. recurrent SDs: $316 \pm 122$ and $240 \pm 89$ vs. $143 \pm 67$ s; Ischemic SC-560 and Ischemic L161,982 vs. Ischemic Vehicle) (Fig. 2D). The coincidence between the duration of depolarization and the associated hyperemia was confirmed by a strong, positive correlation $\left(r=0.825^{* *}\right)$ between these variables (Fig. 2E).

Finally, the early hypoperfusion element of the SD-related CBF response did not appear consistently, therefore measured values (i.e. amplitude, duration) could not be analyzed reliably.

The impact of L161,982 treatment on ischemia outcome. In addition to its vasoconstrictive potential as detailed above, L161,982 administration caused the greatest deviation in all assessed variables, and contributed to the evolution of severe ischemia in a third of the animals assigned to L161,982 treatment (Table 1). Such severe ischemia did not occur in any of the other treatment groups. The most obvious signs of the adverse effect of L161,982 administration were (i) the occurrence of spontaneous SDs within minutes after ischemia onset - and their long duration, (ii) the inability of the tissue to sustain subsequent, triggered SDs, (iii) the lack of detectable CBF response to these SDs, (iv) lower CBF levels at all time points after ischemia onset as compared with any other groups, and (v) occasional, lethal outcome before the termination of the experimental protocol $(\mathrm{n}=2)$. 


\begin{tabular}{|l|c|c|}
\hline & $\begin{array}{c}\text { Experimental protocol } \\
\text { completed (n=8) }\end{array}$ & $\begin{array}{c}\text { Experimental protocol } \\
\text { aborted (n=4) }\end{array}$ \\
\hline Minimum CBF after ischemia onset (\%) & $33.68 \pm 19.75$ & $12.32 \pm 6.96$ \\
\hline Occurrence of spontaneous SD & no (0/8) & yes (3/4) \\
\hline $\begin{array}{l}\text { Duration of first evoked SD (completed } \\
\text { protocol) or spontaneous SD (aborted } \\
\text { protocol) (s) }\end{array}$ & $95 \pm 64$ & no \\
\hline $\begin{array}{l}\text { Successful elicitation of subsequent } \\
\text { recurrent SDs }\end{array}$ & hyperemic & no detectable CBF \\
response
\end{tabular}

Table 1. Two distinct subpopulations of experiments carried out to assess the impact of L161,982 treatment (EP4 receptor antagonism) on SD evolution during ischemia. Data are given as mean \pm stdev. Abbreviations: CBF, cerebral blood flow; SD, spreading depolarization.

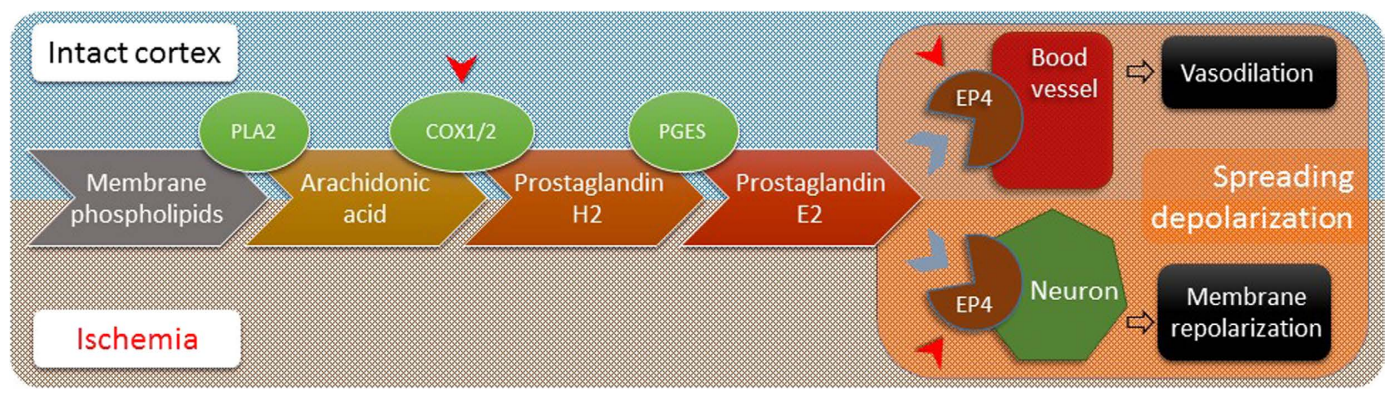

Figure 4. Schematic illustration of the metabolic signaling pathway targeted in the present study, and its suggested involvement in modulating spreading depolarization and the related cerebral blood flow response. Red arrowheads indicate the sites of pharmacological interventions used. Abbreviations: PLA2, phospholipase A2; COX, cyclooxygenase; EP4, receptor type for prostaglandin $\mathrm{E}_{2}$; PGES, prostaglandin E synthase.

\section{Discussion}

Here we set out to discriminate the potential involvement of prostanoids, particularly $\mathrm{PGE}_{2}$ in the regulation of the CBF response to SD. Two experimental approaches have been utilized. First, we selectively inhibited the COX-1 and COX-2 enzymes, which produce the precursor for $\mathrm{PGE}_{2}$ synthesis and thereby regulate the availability of $\mathrm{PGE}_{2}$ (Fig. 4). In support of this approach, the inhibition of COX-2 was previously shown to significantly reduce functional hyperemia in response to whisker stimulation ${ }^{16}$. Next, we pharmacologically blocked the EP4 receptors of $\mathrm{PGE}_{2}$ (Fig. 4), because $\mathrm{EP} 4$ receptors expressed in cerebral arteries were shown to mediate the vasodilatory effect of $\mathrm{PGE}_{2}{ }^{18,30}$, and their activation contributed to functional hyperemia in response to somatosensory stimulation ${ }^{14}$. Because PGE $_{2}$ synthetized via COX-2 suppresses neuronal excitability ${ }^{31}$ and the COX-2 - EP4 signaling pathway has been implicated in the progression of ischemic injury ${ }^{32,33}$, we assessed the impact of the chosen pharmacological treatments on the DC signature of SD, as well. Finally, the neuronal or vascular upregulation of COX-2 and EP4 receptors established in cerebral ischemia ${ }^{29,33}$ prompted us to investigate whether the kinetics of $\mathrm{SD}$ or the related $\mathrm{CBF}$ response are modulated by this signaling pathway in the ischemic brain. This is particularly relevant, as spontaneous, recurrent SDs have been implicated in the progression of ischemic injury $y^{2-5}$.

Of the subsequent phases of the $\mathrm{CBF}$ response to $\mathrm{SD}^{6}$, we focused on the peak hyperemic and post-SD oligemic elements. The first significant observation of the current study is that EP4 receptor antagonism reduced peak hyperemia, and augmented post-SD oligemia of the CBF response to SD in the intact brain (Fig. 3B,C). This indicates that EP4 receptor activation contributes to vasodilation during the CBF response to SD (Fig. 4). It is a novel finding, as the mediation of the hyperemic element of the CBF response by prostanoids was previously thought unlikely, and COX-derived metabolites were attributed a vasoconstrictive rather than a vasodilatory role in the $\mathrm{CBF}$ response to $\mathrm{SD}^{6,26}$. At the same time, our data are consistent with a number of previous reports on the vasodilatory action of EP4 receptor activation by $\mathrm{PGE}_{2}$. For example, antagonism of EP4 receptors inhibited the $\mathrm{PGE}_{2}$-induced vasodilation of the middle meningeal artery of rats in vivo, and of the middle cerebral artery in vitro ${ }^{18,30}$. At the concentration used here, L161,982 previously reduced the NMDA-induced dilation of cerebral arterioles, functional hyperemia to whisker stimulation ${ }^{14}$, and glutamate-evoked dilation of microvessels ${ }^{34}$. Our data thus indicate that EP4 receptor activation achieves CBF elevation in response to SD, as well.

The selective inhibition of COX-1 and COX-2 enzymes exerted no significant, direct effect on any of the elements of the CBF response to SD (Fig. 3), despite the expectation that COX-2 inhibition by NS-398 would decrease the magnitude of the hyperemic element as it did functional hyperemia in response to whisker 
stimulation previously ${ }^{16}$. The reason for the absence of the COX-enzyme related vasodilation may well be that during SD, the activity of COX enzymes also leads to the marked production of vasoconstrictive prostanoids, such as prostaglandin $\mathrm{F} 2 \alpha^{26}$. Inhibition of the COX enzymes as was done in our experiments therefore likely diminishes the SD-related synthesis of both vasoconstrictive and vasodilator prostanoids, causing an undiscernible net outcome on the CBF response. The results of others and of ours together show, that functional hyperemia to neuronal activation is COX-2 dependent (i.e. selective COX-2 inhibition alone reduces the peak of hyperemia) ${ }^{14,16,23}$, while COX-2 inhibition has no clear impact on the CBF response to SD. These data foster the assumption that the CBF response to SD is driven by mechanisms different from physiological neurovascular coupling. Taken prostanoid-based cerebral vasoregulation, it is thus conceivable that vasoconstrictive COX products are released at insignificant concentration during neuronal activation, but their impact is substantial during $\mathrm{SD}^{26}$, obscuring the co-existing vasodilator effect of $\mathrm{PGE}_{2}$.

In the ischemic cortex, our study revealed a considerable reduction of the distinct elements of the CBF response to SD, with no detectable impact of COX enzyme inhibition or EP4 receptor blockade. Consistent with the data presented here, ischemia is known to impair physiological neurovascular coupling as evidenced by the attenuation of functional hyperemia to forepaw stimulation ${ }^{35}$. In addition, in the acute phase of ischemia (the target of investigation here), the abundant release of metabolic mediators of vascular tone, such as adenosine, increased ADP/ATP ratio, lactate accumulation, or the dramatically elevated concentration of extracellular potassium ${ }^{36}$ must obscure potentially still effective, finer signals of vasoregulation. It may also well be that the prostanoid-based regulation of vascular tone becomes more obvious and could prove discernable during the subacute or chronic phases of ischemia, because COX-2 mRNA expression becomes upregulated beginning 6 hours, and endothelial EP4 receptor expression is induced 4 hours after ischemia onset ${ }^{29,37}$.

In summary, EP4 receptor activation contributes to vasodilation during the CBF response to SD in the intact cortex, and is undetectable under the acute phase of ischemia, possibly due to impaired neurovascular coupling, the dominance of metabolic mediators of vascular tone, or the upregulation of the receptors only several hours later.

The present study revealed that the selective pharmacological inhibition of COX-1 or the antagonism of EP4 receptors in the ischemic cortex both hamper the recovery of the nervous tissue from SD (as indicated by increased SD duration, Fig. 2A,B), while the inhibition of COX-2 remained ineffective in this respect. It has been firmly established that COX-2 expression is markedly upregulated during cerebral ischemia, and COX-2 inhibition is neuroprotective - despite cardiovascular side effects ${ }^{38}$. In our hands, COX-2 inhibition was ineffective on the neurophysiological end points taken, most certainly because in this early phase of ischemia COX-2 was not yet upregulated ${ }^{29}$. Instead, the role of COX-1 in promoting repolarization after SD has emerged. These results of ours stand in harmony with the report that ischemia-related hippocampal $\mathrm{PGE}_{2}$ production in a gerbil ischemia model was potently reduced by COX-1 rather than COX-2 inhibition during the early phase of reperfusion in contrast with 24 hours later, when COX-2 appeared to be the dominant source of $\mathrm{PGE}_{2}{ }^{39}$. Despite the daunting complexity of prostanoid signaling, it is tempting to speculate, that COX-1 derived $\mathrm{PGE}_{2}$ should activate neuronal EP4 receptors during SD in the acute phase of ischemia, and thereby promote neuronal repolarization (Fig. 4). This would be an effective means of neuronal survival consistent with previous data demonstrating that longer SD duration predicts more extensive ischemic injury ${ }^{40}$ and genetic inactivation of neuronal EP4 receptors worsens stroke outcome $\mathrm{e}^{33}$. In fact, shortened SD duration mediated by neuronal EP4 receptors could possibly account for smaller infarct volume reported to be achieved by selective EP4 receptor agonism ${ }^{33,41}$.

Even though our results focus on EP4 receptors, $\mathrm{PGE}_{2}$ was shown to achieve neuroprotection by activating the $\mathrm{EP} 2^{42}$ and possibly the EP3 receptors, although there is no consensus on whether EP3 receptor activation is protective under all circumstances ${ }^{43,44}$. It is therefore plausible that COX-1 derived $\mathrm{PGE}_{2}$ could achieve the shortening of SD duration through EP2 and perhaps EP3 receptors, as well.

In the ischemic cortex, the duration of hyperemia in response to SD increased synchronously with elongated SD duration imposed by COX-1 inhibition or EP4 receptor antagonism (Fig. 2B,D). The synchronicity was confirmed by the strong positive correlation between SD and hyperemia duration (Fig. 2E). Earlier we postulated based on similar observations that the return of CBF to baseline after hyperemia may be postponed by the continuing energy need to restore membrane potential ${ }^{10}$. For this reason, it is conceivable that increased hyperemia duration seen as a result of COX-1 inhibition or EP4 receptor antagonism must have been achieved indirectly, by increasing the duration of SD itself.

Here we applied the EP4 receptor blocker L161,982 in a concentration that weakens neurovascular coupling in the intact nervous tissue ${ }^{14,34}$. In addition to our present results that EP4 receptor antagonism delayed tissue recovery after SD during ischemia (see above), we found a major, detrimental impact of EP4 receptor antagonism on ischemia outcome in a subpopulation of animals assigned to the treatment (Table 1). The symptoms revealed both vascular and neurophysiological impairment (i.e. spontaneous SD occurrence with no detectable $\mathrm{CBF}$ response, markedly reduced baseline $\mathrm{CBF}$ during ischemia) indicative of the interaction of these elements, or the potential involvement of both neuronal and vascular EP4 receptors ${ }^{32,33}$. Our data confirm the notion that EP4 receptor activation is necessary for neuronal survival and better ischemia outcome as previously shown by methods including agonism of EP4 receptors, EP4 receptor deletion, and the assessment of EP4 receptor expression in various experimental models of cerebral ischemia ${ }^{32,33,41}$. Even though our data are thus consistent with previous reports, the severe outcome due to L161,982 treatment was unexpected, as neurovascular coupling studies reported no such risk ${ }^{14,34}$ and no previous investigations have evaluated the impact of L161,982 administration on neurophysiological outcome after ischemia. Finally, we suggest that SD evolution may be a critical component of infarct development related to the inactivation of EP4 receptors, as implied by the spontaneous occurrence of long-lasting SD in these animals.

The restriction of SD evolution in neurological intensive care patients has become a central objective, which is driven by the recognition that spontaneously occurring recurrent SDs of long cumulative duration worsen the 
outcome of ischemic or traumatic brain injury ${ }^{4,5}$. So far, the inhibition of NMDA receptors by ketamine administration has been the only strategy of promise to decrease the likelihood of SD occurrence ${ }^{45}$. $\mathrm{PGE}_{2}$ and its specific receptors have long been implicated in mediating neuronal death or survival under ischemia, but have not been associated with SD evolution. The data presented here demonstrate for the first time that the inhibition of COX-1 or the blockade of the EP4 receptors of $\mathrm{PGE}_{2}$ remarkably increases SD duration in the early phase of cerebral ischemia. These significant observations may initiate a new line of investigation to dissect specific components of prostanoid signaling that may play a defining role in sustaining or aborting SD in the ischemic nervous tissue. In the long run, specific elements of prostanoid signaling may present new, effective targets in addition to NMDA receptors to restrict SD evolution and thereby lessen SD-related injury.

\section{Materials and Methods}

The experimental procedures were approved by the National Food Chain Safety and Animal Health Directorate of Csongrád County, Hungary. The procedures conformed to the guidelines of the Scientific Committee of Animal Experimentation of the Hungarian Academy of Sciences (updated Law and Regulations on Animal Protection: 40/2013. (II. 14.) Gov. of Hungary), following the EU Directive 2010/63/EU on the protection of animals used for scientific purposes, and reported in compliance with the ARRIVE guidelines.

Surgical procedures. Surgical procedures were similar to what was previously described ${ }^{46}$. Young adult male Sprague-Dawley rats $(n=60$, body weight: $302 \pm 31 \mathrm{~g})$ were used in the study. The animals were purchased from the Charles River Laboratories, Hungary, were group-housed under a normal 12/12 h light/dark cycle, and had free access to food and drinking water. On the day of experiments, the animals were anesthetized with $1.5-2 \%$ isoflurane in $\mathrm{N}_{2} \mathrm{O}: \mathrm{O}_{2}$ (70\%:30\%), and breathed spontaneously through a head mask throughout the experiment. Body temperature was maintained at $37^{\circ} \mathrm{C}$ with a servo-regulated heating pad. Atropine $(0.1 \%, 0.05 \mathrm{ml})$ was administered intramuscularly shortly before surgical procedures to avoid the production of airway mucus. A catheter was inserted into the left femoral artery to monitor mean arterial blood pressure (MABP) continuously, and arterial blood gas levels at the start of each experimental protocol, and before termination of the experiments.

Next, a midline incision was made in the neck and both common carotid arteries were delicately separated from the surrounding muscle and the vagal nerves. Lidocain (1\%) was administered topically before opening each tissue layer. A silicone coated fishing line used as occluder was looped around each artery for later induction of cerebral ischemia. Rats were transferred to a stereotactic frame and fixed in prone position. Two craniotomies ( $5 \mathrm{~mm}$ lateral from midline, $-3 \mathrm{~mm}$ and $-7 \mathrm{~mm}$ caudal from bregma) were prepared over the right parietal cortex using a dental drill (Technobox, Bien Air 810). A doughnut shape ring of acrylic dental cement was built around the rostral craniotomy for the latter topical application of drugs to the cortical surface. The dura in each craniotomy was carefully dissected, and the craniotomies were regularly irrigated with artificial cerebrospinal fluid (aCSF; mM concentrations: $126.6 \mathrm{NaCl}, 3 \mathrm{KCl}, 1.5 \mathrm{CaCl}_{2}, 1.2 \mathrm{MgCl}, 24.5 \mathrm{NaHCO}_{3}, 6.7$ urea, 3.7 glucose bubbled with $95 \% \mathrm{O}_{2}$ and $5 \% \mathrm{CO}_{2}$ to achieve a constant $\mathrm{pH}$ of 7.4). In the rostral window, an intracortical glass capillary microelectrode (outer tip diameter $=20 \mu \mathrm{m}$ ) was lowered $800-1000 \mu \mathrm{m}$ into the cortex, and an $\mathrm{Ag} / \mathrm{AgCl}$ electrode was implanted under the skin of the animal's neck to be used as reference. In order to assess changes in local CBF, a laser-Doppler needle probe (Probe 402 or 403 connected to Perimed 4001 Master or PeriFlux 5000; Perimed AB, Sweden) was positioned near the cortical surface, adjacent to the intracortical microelectrode, avoiding any large pial vessels. The caudal craniotomoy was later used for SD elicitation.

Electrophysiology and cerebral blood flow measurement. Direct current (DC) potential was recorded via a high input impedance pre-amplifier (NL102G or NL100AK, NeuroLog System, Digitimer Ltd, United Kingdom), connected to a differential amplifier (NL106 or NL107, NeuroLog System, Digitimer Ltd, United Kingdom) with associated filter and conditioner systems (NL125, NL144 or NL530, NeuroLog System, Digitimer Ltd, United Kingdom). Potential line frequency noise $(50 \mathrm{~Hz})$ was removed by a high quality noise eliminator (HumBug, Quest Scientific Instruments Inc., Canada) without any signal attenuation. The resulting signal was digitalized either by an analog/digital (A/D) converter (MP150, Biopac Systems Inc., USA) and continuously acquired at a sampling frequency of $1 \mathrm{kHz}$ or $500 \mathrm{~Hz}$ using the software AcqKnowledge 4.2.0 (Biopac Systems Inc., USA), or another dedicated A/D converter card (NI USB-6008/6009, National Instruments, Austin, Texas, USA) controlled through a custom-made software, written in Labview (National Instruments, Austin, Texas, USA). The laser Doppler signal was digitized and acquired, together with the DC potential, essentially as described above.

Drug administration. Drug solutions or vehicle of equal volume (1.5\% DMSO in $10 \mathrm{ml}$ aCSF) were superfused on the cortical surface free of dura in the rostral cranial window after taking DC potential and CBF baseline for $5 \mathrm{~min}$ under aCSF. Drug concentrations were carefully selected based on dose response curves, selectivity and efficacy reported previously $14,16,18,34$. The following drugs were applied topically: the selective COX-2 inhibitor NS-398 $(100 \mu \mathrm{M} \text {; Cayman })^{16}$, the selective COX-1 inhibitor SC-560 $(25 \mu \mathrm{M} \text {; Cayman })^{22}$, or the selective PGE $_{2}$ receptor (EP4) antagonist L161,982 $(1 \mu \mathrm{M} \text {; Sigma })^{14,34}$. The pharmacological treatment was initiated 40 min prior ischemia induction or the elicitation of the first SD event (i.e. sham-operated group) ${ }^{16}$ and incubation persisted till the end of the experimental protocol.

Ischemia induction and SD elicitation. Following the 40-min incubation period, persistent incomplete global forebrain ischemia was induced in half of the animals, by occluding both common carotid arteries permanently ("2-vessel occlusion", 2VO): occluders were pulled on until resistance was felt and then secured in place. Successful ischemia induction was confirmed by a sharp drop of the laser Doppler signal. As control for ischemia, the remaining animals were used as a sham-operated group (SHAM), in which the occluders were not pulled but left in place. 


\begin{tabular}{|c|c|c|c|}
\hline $\begin{array}{l}\text { Experimental } \\
\text { group }\end{array}$ & Pharmacological treatment & $\begin{array}{l}\text { Ischemia induction } \\
\text { (SHAM or 2VO) }\end{array}$ & n \\
\hline 1 & \multirow{2}{*}{ Vehicle (1.5\% DMSO in aCSF) } & SHAM & 8 \\
\hline 2 & & $2 \mathrm{VO}$ & 7 \\
\hline 3 & \multirow{2}{*}{$100 \mu \mathrm{M}$ NS-398 (COX-2 inhibition) } & SHAM & 7 \\
\hline 4 & & $2 \mathrm{VO}$ & 6 \\
\hline 5 & \multirow{2}{*}{$25 \mu \mathrm{M}$ SC-560 (COX-1 inhibition) } & SHAM & 7 \\
\hline 6 & & $2 \mathrm{VO}$ & 7 \\
\hline 7 & \multirow{2}{*}{$1 \mu \mathrm{M}$ L161,982 (EP4 receptor antagonism) } & SHAM & 6 \\
\hline 8 & & $2 \mathrm{VO}$ & 12 \\
\hline
\end{tabular}

Table 2. Composition of experimental groups. Abbreviations: $2 \mathrm{VO}$, permanent, bilateral common carotid artery occlusion ("two-vessel occlusion"); COX, cyclooxygenase enzyme; DMSO, dimethyl sulfoxide; EP4, receptor for prostaglandin E2; SHAM, sham-operated control for ischemia.

Forty minutes after the start of drug incubation, 4 SDs with an inter-SD interval of 15 minutes were triggered by placing a $1 \mathrm{M} \mathrm{KCl-soaked} \mathrm{cotton} \mathrm{ball} \mathrm{on} \mathrm{the} \mathrm{exposed} \mathrm{cortical} \mathrm{surface} \mathrm{in} \mathrm{the} \mathrm{caudal} \mathrm{cranial} \mathrm{window.} \mathrm{The} \mathrm{cotton}$ ball was removed and the caudal cranial window rinsed with aCSF immediately after each successful SD elicitation. Experiments were terminated by the overdose of the anesthetic agent, $20 \mathrm{~min}$ after triggering the last SD. The combination of pharmacological treatment and ischemia induction resulted in 8 experimental groups (Table 2).

Data analysis. All variables (i.e. DC potential, CBF and MABP) were simultaneously acquired, displayed live, and stored using a personal computer equipped with a dedicated software (AcqKnowledge 4.2 for MP 150, Biopac Systems, Inc., USA). Data analysis was assisted by the inbuilt tools of the same software, or was transferred into a MATLAB environment (MathWorks Inc., USA). Both DC potential and CBF recordings were first downsampled to $1 \mathrm{~Hz}$. The transient negative DC shift indicative of SD was analyzed to assess: amplitude of depolarization, duration of depolarization at half amplitude, area under the curve of the negative DC shift, slope of depolarization, slope of repolarization, and amplitude of post-SD hyperpolarization.

Raw CBF recordings were expressed relative to baseline by using the average CBF value of the first $5 \mathrm{~min}$ under aCSF (100\%) and the recorded biological zero obtained after terminating the experiment ( $0 \%)$ as reference points. Drifting of baseline was determined by sampling a 5-min average 30-35 min after the start of drug incubation, a $10 \mathrm{~s}$ average prior ischemia induction and during the drop after ischemia induction, and 5-min averages prior each SD elicited. The following elements of the SD-related CBF response were characterized: amplitude and duration of early hypoperfusion, amplitude and duration of hyperemia, magnitude of hyperemia (area under the curve), and the amplitude of post-SD oligemia.

The data were evaluated separately for the first SD, and subsequent, recurrent SDs, because of the obvious differences in the kinetics of the SD-associated CBF response. Data of the 3 recurrent SDs were averaged for each animal, thus a single value per animal of each read-out was taken for statistical analysis.

Data are given as mean \pm stdev. The software SPSS (IBM SPSS Statistics for Windows, Version 22.0, IBM Corp.) was used for statistical analysis. A one-way analysis of variance (ANOVA) model was applied for the evaluation of variables derived from the DC potential signature of SD, and the associated CBF response. A repeated measures paradigm was used to evaluate MABP variation and CBF baseline drift. Correlation analysis relied on a one-tailed Pearson test. Levels of significance were defined as $\mathrm{p}<0.05^{*}$ and $\mathrm{p}<0.01 * *$.

\section{References}

1. Leão, A. A. P. Spreading depression of activity in the cerebral cortex. J. Neurophysiol. 7, 359-390 (1944).

2. Hossmann, K. A. Periinfarct depolarizations. Cerebrovasc. Brain Metab. Rev. 8(3), 195-208 (1996).

3. Nedergaard, M. Spreading depression as a contributor to ischemic brain damage. Adv. Neurol. 71, 75-83, discussion 83-84 (1996).

4. Hartings, J. A. et al. The continuum of spreading depolarizations in acute cortical lesion development: re-examining Leão's legacy. J. Cereb. Blood Flow Metab. pii: $0271678 \times 16654495$, ahead of print, (2016).

5. Dreier, J. P. et al. Recording, analysis, and interpretation of spreading depolarizations in neurointensive care: review and recommendations of the COSBID research group. J. Cereb. Blood Flow Metab. pii: $0271678 \times 16654496$, ahead of print, (2016).

6. Ayata, C. \& Lauritzen, M. Spreading depression, spreading depolarizations, and the cerebral vasculature. Physiol. Rev. 95(3), 953-993 (2015)

7. Hoffmann, U. \& Ayata, C. Neurovascular coupling during spreading depolarizations. Acta Neurochir. Suppl. 115, 161-165 (2013).

8. Woitzik, J. et al. Propagation of cortical spreading depolarization in the human cortex after malignant stroke. Neurology 80, 1095-1102 (2013).

9. Feuerstein, D. et al. Regulation of cerebral metabolism during cortical spreading depression. J. Cereb. Blood Flow Metab. in press (2015).

10. Menyhárt, A. et al. High incidence of adverse cerebral blood flow responses to spreading depolarization in the aged ischemic rat brain. Neurobiol. Aging 36, 3269-3277 (2015).

11. Dreier, J. P. The role of spreading depression, spreading depolarization and spreading ischemia in neurological disease. Nat. Med. 17, 439-447 (2011).

12. Attwell, D. et al. Glial and neuronal control of brain blood flow. Nature 468, 232-243 (2010).

13. Cauli, B. \& Hamel, E. Revisiting the role of neurons in neurovascular coupling. Front. Neuroenergetics. 23, 2-9 (2010).

14. Lacroix, A. et al. COX-2-derived prostaglandin E2 produced by pyramidal neurons contributes to neurovascular coupling in the rodent cerebral cortex. J. Neurosci. 35(34), 11791-11810 (2015).

15. Wang, H., Hitron, I. M., Iadecola, C. \& Pickel, V. M. Synaptic and vascular associations of neurons containing cyclooxygenase-2 and nitric oxide synthase in rat somatosensory cortex. Cereb. Cortex 15(8), 1250-1260 (2005). 
16. Niwa, K., Araki, E., Morham, S. G., Ross, M. E. \& Iadecola, C. Cyclooxygenase-2 contributes to functional hyperemia in whiskerbarrel cortex. J. Neurosci. 20(2), 763-770 (2000).

17. Park, J. Y., Pillinger, M. H. \& Abramson, S. B. Prostaglandin E2 synthesis and secretion: the role of PGE2 synthases. Clin. Immunol. 119(3), 229-240 (2006).

18. Myren, M. et al. Functional and molecular characterization of prostaglandin E2 dilatory receptors in the rat craniovascular system in relevance to migraine. Cephalalgia 30(9), 1110-1122 (2010).

19. Howarth, C. The contribution of astrocytes to the regulation of cerebral blood flow. Front. Neurosci. 8, 103 (2014).

20. Petzold, G. C., Albeanu, D. F., Sato, T. F. \& Murthy, V. N. Coupling of neural activity to blood flow in olfactory glomeruli is mediated by astrocytic pathways. Neuron 58(6), 897-910 (2008).

21. Takano, T. et al. Astrocyte-mediated control of cerebral blood flow. Nat. Neurosci. 9(2), 260-267 (2006).

22. Niwa, K., Haensel, C., Ross, M. E. \& Iadecola, C. Cyclooxygenase-1 participates in selected vasodilator responses of the cerebral circulation. Circ. Res. 88(6), 600-608 (2001).

23. Lecrux, C. et al. Pyramidal neurons are "neurogenic hubs" in the neurovascular coupling response to whisker stimulation. J. Neurosci. 31(27), 9836-9847 (2011).

24. Rosenegger, D. G., Tran, C. H., Wamsteeker Cusulin, J. I. \& Gordon, G. R. Tonic local brain blood flow control by astrocytes independent of phasic neurovascular coupling. J. Neurosci. 35(39), 13463-13474 (2015).

25. Lauritzen, M., Hansen, A. J., Kronborg, D. \& Wieloch, T. Cortical spreading depression is associated with arachidonic acid accumulation and preservation of energy charge. J. Cereb. Blood Flow Metab. 10(1), 115-122 (1990).

26. Shibata, M., Leffler, C. W. \& Busija, D. W. Pial arteriolar constriction following cortical spreading depression is mediated by prostanoids. Brain Res. 572(1-2), 190-197 (1992).

27. Fordsmann, J. C. et al. Increased 20-HETE synthesis explains reduced cerebral blood flow but not impaired neurovascular coupling after cortical spreading depression in rat cerebral cortex. J. Neurosci. 33(6), 2562-2570 (2013).

28. Miettinen, S. et al. Spreading depression and focal brain ischemia induce cyclooxygenase-2 in cortical neurons through N-methylD-aspartic acid-receptors and phospholipase A2. Proc. Natl. Acad. Sci. USA 94(12), 6500-6505 (1997).

29. Nogawa, S., Zhang, F., Ross, M. E. \& Iadecola, C. Cyclo-oxygenase-2 gene expression in neurons contributes to ischemic brain damage. J. Neurosci. 17(8), 2746-2755 (1997).

30. Davis, R. J. et al. EP4 prostanoid receptor-mediated vasodilatation of human middle cerebral arteries. Br. J. Pharmacol. 141(4), 580-585 (2004).

31. Yagami, T., Koma, H. \& Yamamoto, Y. Pathophysiological roles of cyclooxygenases and prostaglandins in the central nervous system. Mol. Neurobiol. in press (2015).

32. Andreasson, K. Emerging roles of PGE2 receptors in models of neurological disease. Prostaglandins Other Lipid Mediat. 91(3-4), 104-112 (2010).

33. Liang, X. et al. Signaling via the prostaglandin E2 receptor EP4 exerts neuronal and vascular protection in a mouse model of cerebral ischemia. J. Clin. Invest. 121(11), 4362-4371 (2011).

34. Hall, C. N. et al. Capillary pericytes regulate cerebral blood flow in health and disease. Nature 508(7494), 55-60 (2014).

35. Jackman, K. \& Iadecola, C. Neurovascular regulation in the ischemic brain. Antioxid. Redox. Signal. 22(2), 149-160 (2015).

36. Cippola, M. J. The cerebral circulation. (Morgan \& Claypool Life Sciences, 2009).

37. Li, J. et al. Misoprostol, an anti-ulcer agent and PGE(2) receptor agonist, protects against cerebral ischemia. Neurosci. Lett. 438(2), $210-215(2008)$

38. Iadecola, C. \& Gorelick, P. B. The Janus face of cyclooxygenase-2 in ischemic stroke shifting toward downstream targets. Stroke 36(2), 182-185 (2005).

39. Candelario-Jalil, E. et al. Assessment of the relative contribution of COX-1 and COX-2 isoforms to ischemia-induced oxidative damage and neurodegeneration following transient global cerebral ischemia. J. Neurochem. 86(3), 545-555 (2003).

40. Dijkhuizen, R. M. et al. Correlation between tissue depolarizations and damage in focal ischemic rat brain. Brain Res. 840(1-2), 194-205 (1999).

41. Akram, A., Gibson, C. L. \& Grubb, B. D. Neuroprotection mediated by the $\mathrm{EP}_{4}$ receptor avoids the detrimental side effects of COX-2 inhibitors following ischaemic injury. Neuropharmacology 65, 165-172 (2013).

42. McCullough, L. et al. Neuroprotective function of the PGE2 EP2 receptor in cerebral ischemia. J. Neurosci. 24(1), 257-268 (2004).

43. Ikeda-Matsuo, Y. et al. Microsomal prostaglandin E synthase-1 contributes to ischaemic excitotoxicity through prostaglandin E2 EP3 receptors. Br. J. Pharmacol. 160(4), 847-859 (2010).

44. Ahmad, M. et al. Stimulation of prostaglandin E2-EP3 receptors exacerbates stroke and excitotoxic injury. J. Neuroimmunol. 184(1-2), 172-179 (2007).

45. Sakowitz, O. W. et al. Preliminary evidence that ketamine inhibits spreading depolarizations in acute human brain injury. Stroke 40(8), e519-e522 (2009).

46. Hertelendy, P. et al. Advancing age and ischemia elevate the electric threshold to elicit spreading depolarization in the cerebral cortex of young adult rats. J. Cereb. Blood Flow Metab. in press (2016).

\section{Acknowledgements}

This work was supported by grants from the Hungarian Scientific Research Fund (Grant No. K111923); the Hungarian Brain Research Program (Grant No. KTIA_13_NAP-A-I/13); TÁMOP-4.2.2.D-15/1/KONV-20150024; the Faculty of Medicine, University of Szeged, Hungary; and the Bolyai János Research Scholarship of the Hungarian Academy of Sciences (BO/00327/14/5, to EF).

\section{Author Contributions}

D.P.V. acquisition, analysis and interpretation of data, drafting the article. T.P. acquisition and analysis of data, drafting the article. Á.M. acquisition and interpretation of data, drafting the article. P.H. analysis of data, drafting the article. D.Z.-S. acquisition and analysis of data. R.T. acquisition of data. O.I.-K. acquisition of data. F.B. revising the manuscript critically for important intellectual content, final approval of the version to be published. E.F. substantial contributions to conception and design, drafting the article and revising it critically for important intellectual content, final approval of the version to be published.

\section{Additional Information}

Competing financial interests: The authors declare no competing financial interests.

How to cite this article: Varga, D. P. et al. Contribution of prostanoid signaling to the evolution of spreading depolarization and the associated cerebral blood flow response. Sci. Rep. 6, 31402; doi: 10.1038/srep31402 (2016). 
(c) (i) This work is licensed under a Creative Commons Attribution 4.0 International License. The images or other third party material in this article are included in the article's Creative Commons license, unless indicated otherwise in the credit line; if the material is not included under the Creative Commons license, users will need to obtain permission from the license holder to reproduce the material. To view a copy of this license, visit http://creativecommons.org/licenses/by/4.0/

(C) The Author(s) 2016 